\title{
Quantum periods for 3-dimensional Fano manifolds
}

\author{
TOM COATES \\ Alessio Corti \\ SERGEY GALKIN \\ ALEXANDER KASPRZYK
}

The quantum period of a variety $X$ is a generating function for certain GromovWitten invariants of $X$ which plays an important role in mirror symmetry. We compute the quantum periods of all 3-dimensional Fano manifolds. In particular we show that 3-dimensional Fano manifolds with very ample anticanonical bundle have mirrors given by a collection of Laurent polynomials called Minkowski polynomials. This was conjectured in joint work with Golyshev. It suggests a new approach to the classification of Fano manifolds: by proving an appropriate mirror theorem and then classifying Fano mirrors.

Our methods are likely to be of independent interest. We rework the Mori-Mukai classification of 3-dimensional Fano manifolds, showing that each of them can be expressed as the zero locus of a section of a homogeneous vector bundle over a GIT quotient $V / / G$, where $G$ is a product of groups of the form $\mathrm{GL}_{n}(\mathbb{C})$ and $V$ is a representation of $G$. When $G=\mathrm{GL}_{1}(\mathbb{C})^{r}$, this expresses the Fano 3fold as a toric complete intersection; in the remaining cases, it expresses the Fano 3 -fold as a tautological subvariety of a Grassmannian, partial flag manifold, or projective bundle thereon. We then compute the quantum periods using the quantum Lefschetz hyperplane theorem of Coates and Givental and the abelian/non-abelian correspondence of Bertram, Ciocan-Fontanine, Kim and Sabbah.

14J45, 14J33; 14N35

\section{A Introduction}

The quantum period of a Fano manifold $X$ is a generating function for Gromov-Witten invariants. It is a deformation invariant of $X$ that carries detailed information about quantum cohomology. In this paper we give closed formulas for the quantum periods for all 3-dimensional Fano manifolds. As a consequence we prove a conjecture, made jointly with Golyshev, that identifies Laurent polynomials which correspond under mirror symmetry to each of the 98 deformation families of 3-dimensional Fano 
manifolds with very ample anticanonical bundle. We also exhibit Laurent polynomial mirrors for the remaining 7 deformation families. Our arguments rely on the classification of 3-dimensional Fano manifolds, due to Iskovskikh, Mori and Mukai; this is a difficult theorem whose proof, even today, requires delicate arguments in explicit birational geometry. On the other hand our mirror Laurent polynomials have a simple combinatorial definition and classification. Given a suitable mirror theorem this classification would give a straightforward, combinatorial and uniform alternative proof of the classification of 3-dimensional Fano manifolds. The general outlines of such a mirror theorem are beginning to emerge — see Kontsevich [41], Strominger, Yau and Zaslow [70], Auroux [2;3] and Katzarkov, Kontsevich and Pantev [39] — as are some promising approaches to proving it; see Kontsevich and Soibelman [43; 44], Gross [26] and Gross and Siebert [27; 28; 29].

Let $X$ be a Fano manifold, that is, a smooth projective variety such that the anticanonical bundle $-K_{X}$ is ample. The quantum period $G_{X}(t)$ of $X$, defined in Section B, is a generating function for certain genus-zero Gromov-Witten invariants of $X$. It satisfies a differential equation,

$$
\left(\sum_{k=0}^{r} t^{k} p_{k}(D)\right) G_{X}=0,
$$

where $D=t d / d t$ and the $p_{k}$ are polynomials, called the quantum differential equation for $X$. The quantum differential equation carries information about the quantum cohomology of $X$ : the local system of solutions to the quantum differential equation is an irreducible piece of the restriction of the Dubrovin connection (in the Frobenius manifold given by the quantum cohomology of $X)$ to the line in $H^{\bullet}(X)$ spanned by $c_{1}(X)$. In Sections $1-105$ below we give closed formulas for the quantum periods of the 105 deformation families of 3-dimensional Fano manifolds.

In joint work with Golyshev [11] we introduced Minkowski polynomials: these are a collection of Laurent polynomials $f$ in three variables such that the Newton polytope $\Delta$ of $f$ is a reflexive polytope, defined ${ }^{1}$ in terms of Minkowski decompositions of the facets of $\Delta$. Given a Laurent polynomial $f$, one can define the period of $f$ by

$$
\pi_{f}(t)=\left(\frac{1}{2 \pi \mathrm{i}}\right)^{n} \int_{\left|x_{1}\right|=\cdots=\left|x_{n}\right|=1} \frac{1}{1-t f\left(x_{1}, \ldots, x_{n}\right)} \frac{d x_{1}}{x_{1}} \cdots \frac{d x_{n}}{x_{n}}
$$

\footnotetext{
${ }^{1}$ Some of these Laurent polynomials correspond under mirror symmetry to 3-dimensional Fano manifolds which admit a small toric degeneration; see Batyrev [4]. These Laurent polynomials were considered earlier by Galkin [20; 21].
} 
and this satisfies a differential equation called the Picard-Fuchs equation,

$$
\left(\sum_{k=0}^{r} t^{k} P_{k}(D)\right) \pi_{f}=0,
$$

where the $P_{k}$ are polynomials. There are 3747 Minkowski polynomials (up to monomial change of variables) but Akhtar, Coates, Galkin and Kasprzyk [1] showed that these Laurent polynomials together generate only 165 periods. That is, Minkowski polynomials fall into 165 equivalence classes, where $f$ and $g$ are equivalent if and only if they have the same period. The quantum differential equation (1) of a Kähler manifold has the property that every complex root of the polynomial $p_{0}$ is an integerthis reflects the fact that the quantum cohomology algebra of $X$ carries an integer grading - and we say that a Laurent polynomial $f$ is of manifold type if the PicardFuchs operator (2) has the property that every complex root of $P_{0}$ is an integer. Coates, Galkin and Kasprzyk [14] have computed the Picard-Fuchs operators for the Minkowski polynomials numerically. Their results, which are computer-assisted rigorous and pass a number of stringent checks, show that exactly 98 of the 165 Minkowski periods are of manifold type.

We conjectured, jointly with Golyshev [11], that the 98 Minkowski periods of manifold type ${ }^{2}$ correspond under mirror symmetry to the 98 deformation families of 3 -dimensional Fano manifolds with very ample anticanonical bundle. That is, there is a one-to-one correspondence between deformation families of 3-dimensional Fano manifolds $X$ with very ample anticanonical bundle and equivalence classes of Minkowski polynomials $f$, such that ${ }^{3}$ the Fourier-Laplace transform $\widehat{G}_{X}$ of the quantum period of $X$ coincides with the period $\pi_{f}$ of $f$. Assuming the numerical calculations of Minkowski periods in [14], our results here prove this conjecture.

\section{The classification of Fano 3-folds}

There are exactly 105 deformation families of Fano 3-folds. Of these, 17 parameterise 3 -folds $X$ with Picard rank $\rho(X)=b_{2}(X)=1$. All but one of these 17 families were known to Fano himself. The first modern rank-1 classification, in the style of Fano's double projection from a line, is due to Iskovskikh [36; 37; 38]. More recently, Mukai, in a program announced in [54] and still ongoing, re-proved the rank-1 classification from the study of exceptional vector bundles; see $[55 ; 56 ; 57 ; 58 ; 59 ; 60]$ and Mukai,

\footnotetext{
${ }^{2}$ We expect that the remaining Minkowski periods correspond to smooth 3-dimensional Fano orbifolds.

${ }^{3}$ This is a very weak notion of mirror symmetry. It is natural to conjecture much more: that the Minkowski polynomials $f$ give mirrors to the Fano manifolds $X$ in the sense of Kontsevich's homological mirror symmetry program.
} 
Reid and Takagi [61]. In particular, Mukai gave new model constructions for some of the rank-1 Fano 3-folds as linear sections of homogeneous spaces; we make use of these models below. Mori and Mukai $[50 ; 51 ; 52 ; 53]$ proved that there are precisely 88 families of nonsingular Fano 3 -folds of rank $\geq 2$; their proof was a spectacular display of the power of Mori's then-new theory of extremal rays.

The model constructions given by Mori and Mukai are, however, not well suited for the calculation of quantum periods. Indeed, these model constructions are in terms of extremal rays: typically $X$ is constructed by giving an extremal contraction $f: X \rightarrow Y$, for instance the blow up of some curve in $Y$. For example, consider family number 13 in the table of 3-dimensional Fano manifolds of Picard rank 3 in [53]:

Rank 3, number 13: Mori-Mukai construction $X$ is the blow-up of a hypersurface $W \subset \mathbb{P}^{2} \times \mathbb{P}^{2}$ with centre a curve $C$ of bidegree $(2,2)$ on it such that

$$
C \hookrightarrow W \rightarrow \mathbb{P}^{2} \times \mathbb{P}^{2} \stackrel{p_{i}}{\longrightarrow} \mathbb{P}^{2}
$$

is an embedding for both $i=1,2$, where $p_{i}$ is the projection to the $i^{\text {th }}$ factor of the product $\mathbb{P}^{2} \times \mathbb{P}^{2}$.

This construction, elegant though it is, and natural from the point of view of extremal rays, is not well adapted for doing calculations in Gromov-Witten theory. There are procedures for computing Gromov-Witten invariants of blow-ups - see Gathmann [22], $\mathrm{Hu}$ [33; 34], Lai [45] and Manolache [48] — but, because they are not based on a satisfactory structural understanding of blow-ups on the Gromov-Witten side, they are very difficult to use. Instead, our preferred tools are those for which we have a good structural understanding on the Gromov-Witten side: Givental's mirror theorem [24], the quantum Lefschetz theorem of Coates and Givental [16], and the abelian/nonabelian correspondence of Bertram, Ciocan-Fontanine, Kim, Sabbah [8]. These tools require that $X$ be constructed inside the GIT quotient $F=V / / G$ of a vector space $V$ by the action of a complex Lie group $G$ as the zero-locus of a general section of a homogeneous vector bundle $E \rightarrow V / / G$. Thus we rework the Mori-Mukai classification of 3-dimensional Fano manifolds, proving:

Theorem A.1 Let $X$ be a 3-dimensional Fano manifold. Then there exist

- a vector space $V=\mathbb{C}^{n}$,

- a representation of $G=\prod_{i=1}^{r} \mathrm{GL}_{k_{i}}(\mathbb{C})$ on $V$, and

- a representation $\rho$ of $G$, 
such that $X$ is the vanishing locus, inside a GIT quotient $F=V / / G$ with respect to a suitably chosen stability condition, of a section of the vector bundle $E \rightarrow F$ determined by $\rho$.

We think of $F$ as what Miles Reid would call a key variety: by construction, $F$ is endowed with a universal property characterising the embedding $X \hookrightarrow F$. Both the algebraic geometry and the Gromov-Witten theory of $X$ are inherited from $F$ through the universal property.

The proof of Theorem A.1 occupies a substantial portion of this paper. For many of the 105 families the proof is straightforward; for a few families it is rather tricky. In the majority of cases, $G=\mathrm{GL}_{1}(\mathbb{C})^{r}$ and so $X$ is a complete intersection in a toric variety (and in practice a complete intersection of codimension at most 3 ). Here is a typical example:

Rank 3, number 13: our construction $X$ is the codimension-3 complete intersection in $\mathbb{P}^{2} \times \mathbb{P}^{2} \times \mathbb{P}^{2}$ of general sections of the line bundles $\mathcal{O}(1,1,0), \mathcal{O}(1,0,1)$ and $\mathcal{O}(0,1,1)$.

An immediate consequence of Theorem A.1 is that the moduli space of 3-dimensional Fano manifolds is unirational: the obvious map from $\mathbb{P}\left(H^{0}(F, E)\right)$ to the moduli space of $X$ is dominant.

\section{Highlights}

With our model constructions in hand, we then compute the quantum periods. Most of these calculations are routine, but a number are more interesting. The varieties $\mathrm{MM}_{2-2}$, $\mathrm{MM}_{3-2}$ (Example D.8), $\mathrm{MM}_{3-5}$ and $\mathrm{MM}_{4-2}$ require sophisticated applications of the quantum Lefschetz theorem. Challenging (and new) applications of the abelian/nonabelian correspondence include Theorem F.1, which gives a uniform treatment of seven of the 17 3-dimensional Fano manifolds of rank 1 and the varieties $\mathrm{MM}_{2-17}$, $\mathrm{MM}_{2-20}, \mathrm{MM}_{2-21}, \mathrm{MM}_{2-22}$ and $\mathrm{MM}_{2-26}$.

We draw the reader's attention, too, to Section 106, where we exhibit an example of a high-dimensional Fano manifold with non-unirational moduli space. In essence, this means that there is no explicit ${ }^{4}$ way to write down a general Fano $n$-manifold for $n$ large.

\footnotetext{
${ }^{4}$ Our work here relies on the explicit construction of 3-dimensional Fano manifolds given in Theorem A.1. But we hope that, in the future, a more conceptual approach will be possible. Such an approach is likely to construct Fano manifolds via deformation methods, as in the Gross-Siebert mirror symmetry program $[27 ; 28 ; 29]$, as opposed to explicit descriptions in the style of Theorem A.1.
} 


\section{Perspectives and future directions}

As discussed above, Minkowski polynomials have a combinatorial definition and are classified directly from this definition. Given an appropriate mirror theorem, therefore, we could reverse the perspective of this paper and recover the classification of $3-$ dimensional Fano manifolds from the classification of their mirror Laurent polynomials. Even once such a mirror theorem has been proved, the calculations in this paper are likely to remain a very efficient way in practice to determine the mirror partner to a 3-dimensional Fano manifold. Our results suggest, too, that one should search for higher-dimensional Fano manifolds systematically by searching for their Laurent polynomial mirrors. This is discussed in our joint work with Golyshev [11], where we outline a program to classify 4-dimensional Fano manifolds using these ideas.

We know of no a priori reason why every 3-dimensional Fano manifold admits a construction as in Theorem A.1. At present this can be proven only post-classification, by a case-by-case analysis. The obvious generalization of Theorem A.1 fails in high dimensions (see Section 106 for an example in dimension 66) but it may still hold in low dimensions. In particular, does the generalization of Theorem A.1 hold in dimension 4? For now perhaps the following remarks are not out of place. Since the beginning of the subject people have asked what can birational geometry do for Gromov-Witten theory. For instance a natural question that was asked early on was how do Gromov-Witten invariants transform under birational maps, for instance crepant birational morphisms or blow-ups of nonsingular centres. By now we have learned that these questions are often very subtle; in the case of blow-ups of a smooth centre we have a procedure but not a good structural understanding of the problem. On the other hand, in some areas, we have made good progress in Gromov-Witten calculus, the abelian/non-abelian correspondence being the most general and best example. Perhaps now is the right time to ask what can Gromov-Witten theory do for birational geometry: what view of birational classification do we get if we take seriously ${ }^{5}$ the perspective of the abelian/non-abelian correspondence? Does something like Theorem A.1 hold and, if so, why?

\section{Remarks on the rank 1 case}

As far as we know, most of our constructions of 3-dimensional Fano manifolds of Picard rank $\geq 2$ are new. In Picard rank 1 this is not the case: all of the models that we give are either already in the literature or were known to Mukai. As we have

\footnotetext{
${ }^{5}$ For instance, our model constructions of the 3-dimensional Fano manifolds of Picard rank $\geq 2$ can be used to better organise the calculations of Matsuki [49], which we found very helpful on many occasions. We do not pursue this line here, apart from a few scattered comments in the text below.
} 
said, Mukai gave model constructions for some of the rank-1 3-dimensional Fano manifolds $X$ as linear sections of homogeneous manifolds $G / P$ in their canonical projective embedding. In other words, $X$ is the complete intersection of $G / P \subset \mathbb{P}^{N}$ with a linear subspace of the appropriate codimension in $\mathbb{P}^{N}$. Mukai's models are not always the best for our purposes. The abelian/non-abelian correspondence is currently known to hold only for Lie groups of type $A$, so we prefer to exhibit $X$ as a subvariety of $F=A / / G$, where $G$ is a product of groups of the form $\mathrm{GL}_{k}(\mathbb{C})$. Our rank-1 models are thus in some sense simpler than Mukai's; in each case they either occur as an intermediate step in Mukai's published construction or were known to Mukai.

\section{Remarks on quantum periods of Fano manifolds}

Golyshev [25], based on a heuristic involving mirror symmetry and modular forms, gave a conjectural form of the matrices of small quantum multiplication by the anticanonical class for each of the rank-1 Fano 3-folds, and verified it by explicit calculation of Gromov-Witten numbers (unpublished). This work is the fundamental source of the perspective taken in this paper; it is also an important antecedent to the more precise conjecture (joint with Golyshev) that we prove here. The regularized quantum period of rank-1 Fano 3-folds was computed by Beauville [5], Kuznetsov (unpublished) and Przyjalkowski $[64 ; 65 ; 66]$. Ciolli [10] has computed the small quantum cohomology rings of 13 higher-rank Fano 3-folds.

\section{Plan of the paper}

Sections B-G are devoted to some preliminaries and examples, mostly to fix our notation. In particular we summarise all the results from Gromov-Witten theory that we need. The subsequent Sections 1-105 are self-contained essays, one for each of the deformation families of 3-dimensional Fano manifolds, giving: the standard known model construction; our model construction; a proof that the two constructions coincide; the calculation of the regularized quantum period; and - where appropriate a match with a Minkowski period of manifold type. In more detail: Section B gives the definition of and basic facts about quantum periods; Section $\mathrm{C}$ treats toric Fano manifolds and Givental's mirror theorem; Section D introduces notation for Fano complete intersections in toric varieties and discusses the quantum Lefschetz theorem; Section E provides some geometric constructions and notation that are used in our model constructions; Section F summarizes the abelian/non-abelian correspondence; and in Section $\mathrm{G}$ we compute the quantum periods for Fano manifolds of dimensions 1 and 2. The appendix describes a table, given in an online supplement [12], that exhibits Laurent polynomial mirrors for each of the 105 deformation families of 3-dimensional Fano manifolds. 
Acknowledgements This research was supported by a Royal Society University Research Fellowship (TC); ERC Starting Investigator Grant number 240123; the Leverhulme Trust; AG Laboratory NRU-HSE, RF government grant, ag. 11.G34.31.0023; EPSRC grant EP/I008128/1; JSPS Grant-in-Aid for Scientific Research 10554503; the WPI Initiative of MEXT, Japan; NSF Grant DMS-0600800; NSF FRG Grant DMS0652633; FWF Grant P20778; and the ERC Advanced Grant GEMIS. Galkin thanks Bumsig Kim and Alexander Kuznetsov for useful conversations.

\section{B The $J$-function and the quantum period}

Let $X$ be a smooth projective variety over $\mathbb{C}$. For $\beta \in H_{2}(X ; \mathbb{Z})$, let $X_{0,1, \beta}$ denote the moduli space of degree- $\beta$ stable maps to $X$ from genus-zero curves with one marked point (see Kontsevich [40] and Kontsevich and Manin [42]); let $\left[X_{0,1, \beta}\right]^{\text {vir }}$ in $H_{\bullet}\left(X_{0,1, \beta} ; \mathbb{Q}\right)$ denote the virtual fundamental class of $X_{0,1, \beta}$ (see Li and Tian [47] and Behrend and Fantechi [7]); let ev: $X_{0,1, \beta} \rightarrow X$ denote the evaluation map at the marked point; and let $\psi \in H^{2}\left(X_{0,1, \beta} ; \mathbb{Q}\right)$ denote the first Chern class of the universal cotangent line at the marked point. The $J$-function of $X$ is a generating function for genus-zero Gromov-Witten invariants of $X$ :

$$
J_{X}(\sigma+\tau)=e^{\sigma / z} e^{\tau / z}\left(1+\sum_{\substack{\beta \in H_{2}(X ; \mathbb{Z}) \\ \beta \neq 0}} Q^{\beta} e^{\langle\beta, \tau\rangle} \mathrm{ev}_{\star}\left(\left[X_{0,1, \beta}\right]^{\mathrm{vir}} \cap \frac{1}{z(z-\psi)}\right)\right) .
$$

Here $\sigma \in H^{0}(X ; \mathbb{Q}), \tau \in H^{2}(X ; \mathbb{Q}), Q^{\beta}$ is the representative of $\beta$ in the group ring $\mathbb{Q}\left[H_{2}(X ; \mathbb{Z})\right]$ and we expand $1 /(z(z-\psi))$ as the series $\sum_{k \geq 0} z^{-k-2} \psi^{k}$. Let $\Lambda_{X}$ denote the completion of $\mathbb{Q}\left[H_{2}(X ; \mathbb{Z})\right]$ with respect to the valuation $v$ defined by $v\left(Q^{\beta}\right)=\langle\beta, \omega\rangle$, where $\omega$ is the Kähler class of $X$. The $J$-function is a function on $H^{0}(X ; \mathbb{Q}) \oplus H^{2}(X ; \mathbb{Q})$ that takes values in $H^{\bullet}\left(X ; \Lambda_{X}\right) \llbracket z^{-1} \rrbracket$. It plays a key role in mirror symmetry; see Givental [23; 24] and Cox and Katz [18]. We have

$$
J_{X}(\sigma+\tau)=1+(\sigma+\tau) z^{-1}+O\left(z^{-2}\right),
$$

where 1 is the unit element in $H^{\bullet}(X)$.

Suppose now that $X$ is a Fano manifold, ie a smooth projective variety over $\mathbb{C}$ such that the anticanonical line bundle $-K_{X}$ is ample. Consider the component of the $J$-function $J_{X}(\sigma+\tau)$ along the unit class $1 \in H^{\bullet}(X ; \mathbb{Q})$. Set $\sigma=\tau=0$ and $z=1$ and replace $Q^{\beta} \in \Lambda_{X}$ by $t^{\left\langle\beta,-K_{X}\right\rangle}$. The resulting formal power series in the variable $t$ 
is called the quantum period of $X$,

$$
G_{X}(t)=1+\sum_{d \geq 2} \sum_{\substack{\beta \in H_{2}(X ; \mathbb{Z}) \\\left\langle\beta,-K_{X}\right\rangle=d}} t^{d}\left\langle\phi_{\mathrm{vol}} \cdot \psi^{d-2}\right\rangle_{0,1, \beta}^{X},
$$

where $\phi_{\mathrm{vol}}$ is a top-degree cohomology class on $X$ such that $\int_{X} \phi_{\mathrm{vol}}=1$ and the correlator denotes a Gromov-Witten invariant:

$$
\left\langle\phi_{\mathrm{vol}} \cdot \psi^{d-2}\right\rangle_{0,1, \beta}^{X}=\int_{\left[X_{0,1, \beta}\right]^{\mathrm{vir}}} \mathrm{ev}^{\star}\left(\phi_{\mathrm{vol}}\right) \cup \psi^{d-2} .
$$

Write the quantum period as

$$
G_{X}(t)=1+\sum_{d \geq 2} c_{d} t^{d}
$$

The regularized quantum period of $X$ is

$$
\widehat{G}_{X}(t)=1+\sum_{d \geq 2} d ! c_{d} t^{d}
$$

\section{B.1 The big $J$-function and the small $J$-function}

Our $J$-function $J_{X}(t)$ is sometimes called the "small $J$-function"; it coincides with the $J$-function defined by Givental [24]. For the small $J$-function $J_{X}(t)$, the parameter $t$ is taken to lie in $H^{0}(X) \oplus H^{2}(X)$. Other authors consider a "big J-function" $J(t)$, where the parameter $t$ ranges over all of $H^{\bullet}(X)$. The big $J$-functions $J(t)$ considered by Coates and Givental, and Ciocan-Fontanine, Kim and Sabbah, coincide with our $J_{X}(t)$, except for an overall factor of $z$, when $t$ is restricted to lie in $H^{0}(X) \oplus H^{2}(X)$ : to see this, apply the string equation and the divisor equation — see Pandharipande [63, Section 1.2] — to the definition of the big $J$-function [16, Equation 11; 9, Equation 5.2.1]. The overall factor of $z$ here comes from an unfortunate mismatch of conventions.

\section{Fano and nef toric manifolds}

Let $T=\left(\mathbb{C}^{\times}\right)^{r}$. Write $\mathbb{L}=\operatorname{Hom}\left(\mathbb{C}^{\times}, T\right)$ for the lattice of subgroups of $T$ and write $\mathbb{L}^{\vee}$ for the dual lattice $\operatorname{Hom}\left(T, \mathbb{C}^{\times}\right)$. Elements of $\mathbb{L}^{\vee}$ are characters of $T$. Consider an $r \times N$ integer matrix $M$ of rank $r$ whose columns span a strictly convex cone $\mathcal{C}$ in $\mathbb{R}^{r}$. The columns of $M$ define characters of $T$, via the canonical isomorphism $\mathbb{L}^{\vee} \cong \mathbb{Z}^{r}$ and hence determine an action of $T$ on $\mathbb{C}^{N}$. Given a stability condition $\omega \in \mathbb{L}^{\vee} \otimes \mathbb{R}$ we can form the GIT quotient:

$$
X_{\omega}:=\mathbb{C}^{N} / / \omega T \text {. }
$$


Any smooth projective toric variety $X$ arises via this construction for some choice of $M$ and $\omega$; we refer to the matrix $M$ as weight data for $X$ and to $\omega$ as a stability condition for $X$.

There is a wall-and-chamber decomposition of $\mathcal{C} \subset \mathbb{L}^{\vee} \otimes \mathbb{R}$, called the secondary fan, and if stability conditions $\omega_{1}$ and $\omega_{2}$ lie in the same chamber then the GIT quotients $X_{\omega_{1}}$ and $X_{\omega_{2}}$ coincide. Write $c_{i} \in \mathbb{L}^{\vee}$ for the $i^{\text {th }}$ column of $M$ and $\left\langle c_{i_{1}}, \ldots, c_{i_{k}}\right\rangle$ for the $\mathbb{R}_{\geq 0}$-span of the columns $c_{i_{1}}, \ldots, c_{i_{k}}$. The walls of the secondary fan are given by all cones of the form $\left\langle c_{i_{1}}, \ldots, c_{i_{k}}\right\rangle$ that have dimension $r-1$. The chambers of the secondary fan are the connected components of the complement of the walls; these are $r$-dimensional open cones in $\mathcal{C} \subset \mathbb{L}^{\vee} \otimes \mathbb{R}$. We always take our stability condition $\omega$ to lie in a chamber. Given such an $\omega$, the irrelevant ideal $I_{\omega} \subset \mathbb{C}\left[x_{1}, \ldots, x_{N}\right]$ is

$$
I_{\omega}=\left(x_{i_{1}} x_{i_{2}} \cdots x_{i_{r}} \mid \omega \in\left\langle c_{i_{1}}, \ldots, c_{i_{r}}\right\rangle\right)
$$

and the unstable locus is $V\left(I_{\omega}\right) \subset \mathbb{C}^{N}$. The GIT quotient $X_{\omega}$ is

$$
X_{\omega}=\left(\mathbb{C}^{N} \backslash V\left(I_{\omega}\right)\right) / T .
$$

The variety $X_{\omega}$ is nonsingular if and only if $\left\{c_{i_{1}}, \ldots, c_{i_{r}}\right\}$ is an integer basis for $\mathbb{L}^{\vee}$ for each $\left\{i_{1}, \ldots, i_{r}\right\}$ such that $\omega \in\left\langle c_{i_{1}}, \ldots, c_{i_{r}}\right\rangle$.

Suppose now that $M$ and $\omega$ are weight data and a stability condition for $X$, respectively. A character $\xi \in \mathbb{L}^{\vee}$ defines a line bundle $L_{\xi}$ on $X$ and hence a cohomology class $c_{1}\left(L_{\xi}\right) \in H^{2}(X ; \mathbb{Q})$. Thus the columns of $M$ define cohomology classes $D_{1}, \ldots, D_{N} \in H^{2}(X ; \mathbb{Q})$. Define the $I-$ function of $X$ by

$$
I_{X}(\tau)=e^{\tau / z} \sum_{\beta \in H_{2}(X ; \mathbb{Z})} Q^{\beta} e^{\langle\beta, \tau\rangle} \frac{\prod_{i=1}^{N} \prod_{m \leq 0} D_{i}+m z}{\prod_{i=1}^{N} \prod_{m \leq\left\langle\beta, D_{i}\right\rangle} D_{i}+m z} .
$$

Here, $\tau \in H^{2}(X ; \mathbb{Q})$ and $Q^{\beta}$ is, as before, the representative of $\beta$ in the group ring $\mathbb{Q}\left[H_{2}(X ; \mathbb{Z})\right]$. The $I$-function $I_{X}$ is a function on $H^{2}(X ; \mathbb{Q})$ that takes values in $H^{\bullet}\left(X ; \Lambda_{X}\right) \llbracket z^{-1} \rrbracket$. Note that all but finitely many terms in the infinite products cancel and that

$$
\frac{1}{D_{i}+m z}=\frac{1}{m z}-\frac{D_{i}}{(m z)^{2}}+\frac{D_{i}^{2}}{(m z)^{3}}+\cdots
$$

is well defined as an element of $H^{\bullet}(X) \llbracket z^{-1} \rrbracket$.

Theorem C.1 (Givental) Let $X$ be a toric manifold such that $-K_{X}$ is nef. Then

$$
J_{X}(\theta(\tau))=I_{X}(\tau)
$$


for some function $\theta: H^{2}(X ; \mathbb{Q}) \rightarrow H^{0}\left(X ; \Lambda_{X}\right) \oplus H^{2}\left(X ; \Lambda_{X}\right)$. Furthermore, the function $\theta$ is uniquely determined by the expansion

$$
I_{X}(\tau)=1+\theta(\tau) z^{-1}+O\left(z^{-2}\right) .
$$

If $X$ is Fano then $\theta(\tau)=\tau$.

Proof This follows immediately from Givental's mirror theorem for toric varieties [24].

Corollary C.2 Let $X$ be a Fano toric manifold and let $D_{1}, \ldots, D_{N} \in H^{2}(X ; \mathbb{Q})$ be the cohomology classes of the torus-invariant divisors on $X$. The quantum period of $X$ is

$$
G_{X}(t)=\sum_{\substack{\beta \in H_{2}(X ; \mathbb{Z}) \\ \forall i\left\langle\beta, D_{i}\right\rangle \geq 0}} \frac{t^{\left\langle\beta,-K_{X}\right\rangle}}{\prod_{i=1}^{N}\left\langle\beta, D_{i}\right\rangle !} .
$$

Proof The quantum period $G_{X}$ is obtained from the component of the $J$-function $J_{X}(\tau)$ along the unit class $1 \in H^{\bullet}(X ; \mathbb{Q})$ by setting $\tau=0, z=1$ and $Q^{\beta}=t^{\left\langle\beta,-K_{X}\right\rangle}$. Now apply Theorem C.1.

Example C.3 (number 36 on the Mori-Mukai list of 3-dimensional Fano manifolds of rank 2) Here $X$ is the projective bundle $\mathbb{P}(\mathcal{O} \oplus \mathcal{O}(2))$ over $\mathbb{P}^{2}$. This is a toric variety with weight data

$$
\begin{array}{rrrrrc}
1 & 1 & 1 & -2 & 0 & L \\
0 & 0 & 0 & 1 & 1 & M
\end{array}
$$

and nef cone Nef $X$ spanned by $L$ and $M$. The $L$ and $M$ next to the weight data here denote the line bundles associated to the standard basis of $\mathbb{L}^{\vee}=\mathbb{Z}^{2}$; we use this notation, and its natural extension to the case where $\mathbb{L}^{\vee}=\mathbb{Z}^{r}$ with $r \neq 2$, freely throughout the paper. Corollary C.2 yields

$$
G_{X}(t)=\sum_{d_{1}=0}^{\infty} \sum_{d_{2}=2 d_{1}}^{\infty} \frac{t^{d_{1}+2 d_{2}}}{\left(d_{1} !\right)^{3}\left(d_{2}-2 d_{1}\right) ! d_{2} !}
$$

and regularizing gives

$$
\widehat{G}(t)=1+2 t^{2}+6 t^{4}+60 t^{5}+20 t^{6}+840 t^{7}+70 t^{8}+7560 t^{9}+\cdots .
$$




\section{Fano toric complete intersections}

Assumptions D.1 Throughout Section D, take $Y$ to be a smooth projective toric variety such that $-K_{Y}$ is nef and take $X$ to be a smooth Fano complete intersection in $Y$ defined by a section of $E=L_{1} \oplus \cdots \oplus L_{S}$, where each $L_{i}$ is a nef line bundle. Let $\rho_{i}=c_{1}\left(L_{i}\right)$ and let $\Lambda=\rho_{1}+\cdots+\rho_{s}$. By the adjunction formula,

$$
-K_{X}=\left.\left(-K_{Y}-\Lambda\right)\right|_{X}
$$

We assume that the line bundle $-K_{Y}-\Lambda$ on $Y$ is nef on $Y$, that is, we assume that $\left\langle\beta,-K_{Y}-\Lambda\right\rangle \geq 0$ for all $\beta$ in the Mori cone of $Y$.

\section{D.1 The quantum Lefschetz theorem}

We will compute the quantum period of $X$ by computing certain twisted GromovWitten invariants of the ambient space $Y$ using the quantum Lefschetz theorem of Coates and Givental [16]. Consider the $\mathbb{C}^{\times}$-action on the total space of $E$ given by rescaling fibres (with the trivial action on the base). Let $\lambda$ denote the first Chern class of the line bundle $\mathcal{O}(1)$ over $\mathbb{C} P^{\infty} \cong B \mathbb{C}^{\times}$, so that the $\mathbb{C}^{\times}$-equivariant cohomology of a point is $\mathbb{Q}[\lambda]$, and let $\boldsymbol{e}(\cdot)$ denote the $\mathbb{C}^{\times}$-equivariant Euler class. Coates and Givental [16] define a complex of $\mathbb{C}^{\times}$-equivariant sheaves $E_{0,1, \beta}$ on $Y_{0,1, \beta}$. In this case $E_{0,1, \beta}$ is a $\mathbb{C}^{\times}$-equivariant vector bundle over $Y_{0,1, \beta}$, and there is a $\mathbb{C}^{\times}$equivariant evaluation map $E_{0,1, \beta} \rightarrow \mathrm{ev}^{\star} E$. Let $E_{0,1, \beta}^{\prime}$ be the kernel of this evaluation map. The twisted $J$-function is

$$
\begin{aligned}
& J_{\boldsymbol{e}, E}(\sigma+\tau) \\
& =e^{\sigma / z} e^{\tau / z}\left(1+\sum_{\substack{\beta \in H_{2}(Y ; \mathbb{Z}) \\
\beta \neq 0}} Q^{\beta} e^{\langle\beta, \tau\rangle} \mathrm{ev}_{\star}\left(\left[Y_{0,1, \beta}\right]^{\mathrm{vir}} \cap e\left(E_{0,1, \beta}^{\prime}\right) \cap \frac{1}{z(z-\psi)}\right)\right) .
\end{aligned}
$$

Here $\sigma \in H^{0}(Y ; \mathbb{Q}), \tau \in H^{2}(Y ; \mathbb{Q}), Q^{\beta}$ is the representative of $\beta$ in the group ring $\mathbb{Q}\left[H_{2}(Y ; \mathbb{Z})\right]$ and we expand $1 /(z(z-\psi))$ as the series $\sum_{k \geq 0} z^{-k-2} \psi^{k}$. The twisted $J$-function is ${ }^{6}$ a function on $H^{0}(Y ; \mathbb{Q}) \oplus H^{2}(Y ; \mathbb{Q})$ that takes values in $H^{\bullet}\left(Y ; \Lambda_{Y}[\lambda]\right) \llbracket z^{-1} \rrbracket$. It satisfies

$$
J_{\boldsymbol{e}, E}(\sigma+\tau)=1+(\sigma+\tau) z^{-1}+O\left(z^{-2}\right),
$$

${ }^{6}$ Coates and Givental consider a "big twisted $J$-function" $J_{\boldsymbol{e}, E}(t)$, where the parameter $t$ ranges over all of $H^{\bullet}(X)$. Exactly as in Section B.1, this coincides with our twisted $J$-function, up to an overall factor of $z$, when $t$ is restricted to lie in $H^{0}(X) \oplus H^{2}(X)$. 
where 1 is the unit element in $H^{\bullet}(Y)$. The twisted $J$-function admits a non-equivariant limit $J_{Y, X}$, which satisfies

$$
j_{\star} J_{X}\left(j^{\star}(\sigma+\tau)\right)=J_{Y, X}(\sigma+\tau) \cup \prod_{i=1}^{s} \rho_{i} .
$$

Here, $j: X \rightarrow Y$ is the inclusion and the equality holds after applying the homomorphism between $\Lambda_{X}$ and $\Lambda_{Y}$ induced by $j$. Since we can determine the quantum period $G_{X}$ from the component of $J_{X}$ along the unit class $1 \in H^{\bullet}(X)$, we can determine $G_{X}$ from the component of $J_{Y, X}$ along the unit class $1 \in H^{\bullet}(Y)$.

The quantum Lefschetz theorem determines the twisted $J$-function $J_{\boldsymbol{e}, E}$ from the twisted $I$-function

$$
I_{\boldsymbol{e}, E}(\tau)=\sum_{\beta \in H_{2}(Y ; \mathbb{Z})} Q^{\beta} J_{\beta}(\tau) \prod_{i=1}^{s} \prod_{m=1}^{\left\langle\beta, \rho_{i}\right\rangle}\left(\lambda+\rho_{i}+m z\right),
$$

where

$$
J_{Y}(\tau)=\sum_{\beta \in H_{2}(Y ; \mathbb{Z})} Q^{\beta} J_{\beta}(\tau)
$$

and so, in particular, $J_{0}(\tau)=e^{\tau / z}$.

Proposition D.2 Under Assumptions D.1, we have

$$
I_{\boldsymbol{e}, E}(\tau)=A(\tau)+B(\tau) z^{-1}+O\left(z^{-2}\right)
$$

for some functions

$$
\begin{aligned}
& A: H^{2}(Y ; \mathbb{Q}) \rightarrow H^{0}\left(Y ; \Lambda_{Y}\right) \\
& B: H^{2}(Y ; \mathbb{Q}) \rightarrow H^{0}\left(Y ; \Lambda_{Y}[\lambda]\right) \oplus H^{2}\left(Y ; \Lambda_{Y}[\lambda]\right) .
\end{aligned}
$$

If $-K_{X}$ is the restriction of an ample line bundle on $Y$, ie if $\left\langle\beta,-K_{Y}-\Lambda\right\rangle>0$ for all $\beta$ in the Mori cone of $Y$, then $A$ is the constant function with value the unit class $1 \in H^{0}(Y ; \mathbb{Q})$ and $B(\tau)=\tau+C(\tau) 1$ with

$$
C(\tau)=\sum_{\substack{\beta \in H_{2}(Y ; \mathbb{Z}) \\\left\langle\beta,-K_{Y}-\Lambda\right\rangle=1}} n_{\beta} Q^{\beta} e^{\langle\beta, \tau\rangle}
$$

for some rational numbers $n_{\beta}$. In general we have

$$
A \equiv 1 \bmod \left\{Q^{\beta} \mid \beta \neq 0, \beta \text { in the Mori cone of } Y\right\} .
$$


Proof Combine the fact that $J_{0}(\tau)=e^{\tau / z}=1+\tau z^{-1}+O\left(z^{-2}\right)$ with the fact that $I_{\boldsymbol{e}, E}$ is homogeneous of degree zero with respect to the grading $\operatorname{deg} Q^{\beta}=\left\langle\beta,-K_{Y}-\Lambda\right\rangle, \quad \operatorname{deg} z=1, \quad \operatorname{deg} \lambda=1, \quad \operatorname{deg} \phi=k \quad$ if $\phi \in H^{2 k}(Y ; \mathbb{Q})$. With respect to this grading, $A(\tau)$ is homogeneous of degree zero and $B(\tau)$ is homogeneous of degree one.

Theorem D.3 Under Assumptions D.1, with $A, B$ and $C$ as in Proposition D.2, we have

$$
J_{\boldsymbol{e}, E}(\theta(\tau))=\frac{I_{\boldsymbol{e}, E}(\tau)}{A(\tau)}, \quad \text { where } \theta(\tau)=\frac{B(\tau)}{A(\tau)} .
$$

If $-K_{X}$ is the restriction of an ample class on $Y$ then $J_{\boldsymbol{e}, E}(\tau)=e^{-C(\tau) / z} I_{\boldsymbol{e}, E}(\tau)$.

Proof The first statement is a slight generalization of Corollary 7 in Coates and Givental [16] and is proved in exactly the same way. When $-K_{X}$ is the restriction of an ample class on $Y$, combining the first statement with Proposition D.2 gives

$$
J_{\boldsymbol{e}, E}(\tau+C(\tau) 1)=I_{\boldsymbol{e}, E}(\tau) .
$$

The string equation [63, Section 1.2] now implies that

$$
J_{\boldsymbol{e}, E}(\tau+C(\tau) 1)=e^{C(\tau) / z} J_{\boldsymbol{e}, E}(\tau),
$$

completing the proof.

The twisted $I$-function admits a non-equivariant limit,

$$
I_{Y, X}(\tau)=\sum_{\beta \in H_{2}(Y ; \mathbb{Z})} Q^{\beta} J_{\beta}(\tau) \prod_{i=1}^{s} \prod_{m=1}^{\left\langle\beta, \rho_{i}\right\rangle}\left(\rho_{i}+m z\right) .
$$

Corollary D.4 Under Assumptions D.1, with $A, B$ and $C$ as in Proposition D.2, we have

$$
I_{Y, X}(\tau)=A(\tau)+B^{\prime}(\tau) z^{-1}+O\left(z^{-2}\right),
$$

where $B^{\prime}(\tau)=\left.B(\tau)\right|_{\lambda=0}$, and

$$
J_{Y, X}(\theta(\tau))=\frac{I_{Y, X}(\tau)}{A(\tau)}, \quad \text { where } \theta(\tau)=\frac{B^{\prime}(\tau)}{A(\tau)} .
$$

If $-K_{X}$ is the restriction of an ample class on $Y$ then $J_{Y, X}(\tau)=e^{-C(\tau) / z} I_{Y, X}(\tau)$.

Proof Take the non-equivariant limit $\lambda \rightarrow 0$ of Proposition D.2 and Theorem D.3. 
Corollary D.5 Let the toric complete intersection $X$ and the toric variety $Y$ be such that Assumptions D.1 holds. Let $D_{1}, \ldots, D_{N} \in H^{2}(Y ; \mathbb{Q})$ be the cohomology classes of the torus-invariant divisors on $Y$ and let the classes $\rho_{i}$ and $\Lambda=\rho_{1}+\cdots+\rho_{s}$ be as in Assumptions D.1. Suppose that the line bundles $-K_{Y}$ and $-K_{Y}-\Lambda$ on $Y$ are ample. Then the quantum period of $X$ is

$$
G_{X}(t)=e^{-c t} \sum_{\substack{\beta \in H_{2}(Y ; \mathbb{Z}) \\ \forall i\left\langle\beta, D_{i}\right\rangle \geq 0}} t^{\left\langle\beta,-K_{Y}-\Lambda\right\rangle} \frac{\prod_{j=1}^{s}\left\langle\beta, \rho_{j}\right\rangle !}{\prod_{i=1}^{N}\left\langle\beta, D_{i}\right\rangle !},
$$

where $c$ is the unique rational number such that the right-hand side has the form $1+O\left(t^{2}\right)$.

Proof Recall that $G_{X}$ is obtained from the component of the $J$-function $J_{X}(\sigma+\tau)$ along the unit class $1 \in H^{\bullet}(X ; \mathbb{Q})$ by setting $\sigma=\tau=0, z=1$ and $Q^{\beta}=t^{\left\langle\beta,-K_{X}\right\rangle}$. In view of $(8)$, we need the component of $J_{Y, X}(0)$ along $1 \in H^{\bullet}(Y ; \mathbb{Q})$. Computing $J_{Y}(\tau)$ using Theorem C.1, we see that

$$
I_{Y, X}(\tau)=e^{\tau / z} \sum_{\beta \in H_{2}(X ; \mathbb{Z})} Q^{\beta} e^{\langle\beta, \tau\rangle} \frac{\prod_{i=1}^{N} \prod_{m \leq 0} D_{i}+m z}{\prod_{i=1}^{N} \prod_{m \leq\left\langle\beta, D_{i}\right.} D_{i}+m z} \prod_{j=1}^{s} \prod_{m=1}^{\left\langle\beta, \rho_{j}\right\rangle}\left(\rho_{j}+m z\right) .
$$

Applying Corollary D.4, we see that the component of $J_{Y, X}(\tau)$ along $1 \in H^{\bullet}(Y ; \mathbb{Q})$ is

$$
e^{-C(\tau) / z} \sum_{\substack{\beta \in H_{2}(Y ; \mathbb{Z}) \\ \forall i\left\langle\beta, D_{i}\right\rangle \geq 0}} Q^{\beta} e^{\langle\beta, \tau\rangle} \frac{\prod_{j=1}^{s} \prod_{m=1}^{\left\langle\beta, \rho_{j}\right\rangle}(m z)}{\prod_{i=1}^{N} \prod_{1 \leq m \leq\left\langle\beta, D_{i}\right\rangle}(m z)},
$$

where

$$
C(\tau)=\sum_{\substack{\beta \in H_{2}(Y ; \mathbb{Z}) \\\left\langle\beta,-K_{Y}-\Lambda\right\rangle=1}} n_{\beta} Q^{\beta} e^{\langle\beta, \tau\rangle}
$$

for rational numbers $n_{\beta}$ as in Proposition D.2. Setting $\tau=0, z=1$ and $Q^{\beta}=$ $t^{\left\langle\beta,-K_{Y}-\Lambda\right\rangle}$ yields

$$
G_{X}(t)=e^{-c t} \sum_{\substack{\beta \in H_{2}(Y ; \mathbb{Z}): \\ \forall i\left\langle\beta, D_{i}\right\rangle \geq 0}} t^{\left\langle\beta,-K_{Y}-\Lambda\right\rangle} \frac{\prod_{j=1}^{s}\left\langle\beta, \rho_{j}\right\rangle !}{\prod_{i=1}^{N}\left\langle\beta, D_{i}\right\rangle !}
$$

for some rational number $c$. We saw in Section B that the right-hand side has no linear term in $t$; this determines $c$. 
Remark D.6 Comparing Corollary D.5 with Corollary C.2, we see that if each of the line bundles $L_{1}, \ldots, L_{S}$ in Corollary D.5 is a tensor power or fractional tensor power of $-K_{Y}$ then we can compute the quantum period $G_{X}$ from the quantum period $G_{Y}$ and the line bundles $L_{i}$ alone, without needing to know the full $J$-function $J_{Y}$.

Example D.7 Let $X$ be the divisor on $Y=\mathbb{P}^{2} \times \mathbb{P}^{2}$ of bidegree $(2,2)$. The toric variety $Y$ has weight data

$$
\begin{aligned}
& \begin{array}{llllllll}
1 & 1 & 1 & 0 & 0 & 0 & L
\end{array} \\
& \begin{array}{llllllll}
0 & 0 & 0 & 1 & 1 & 1 & M
\end{array}
\end{aligned}
$$

and the nef cone Nef $Y$ is spanned by $L$ and $M$. The variety $X$ is a member of the ample linear system $|2 L+2 M|$, and $-\left(K_{Y}+X\right) \sim L+M$ is ample. Corollary D.5 yields

$$
G_{X}(t)=e^{-4 t} \sum_{l=0}^{\infty} \sum_{m=0}^{\infty} t^{l+m} \frac{(2 l+2 m) !}{(l !)^{3}(m !)^{3}}
$$

and regularizing gives

$$
\begin{aligned}
\widehat{G}_{X}(t)=1+44 t^{2}+528 t^{3} & +11292 t^{4}+228000 t^{5}+4999040 t^{6} \\
+ & 112654080 t^{7}+2613620380 t^{8}+61885803840 t^{9}+\cdots .
\end{aligned}
$$

Example D.8 Let $F$ be the toric variety with weight data

$$
\begin{array}{rrrrrrrc}
1 & 1 & 0 & 0 & -1 & 0 & 0 & L \\
0 & 0 & 1 & 1 & -1 & 0 & 0 & M \\
0 & 0 & 0 & 0 & 1 & 1 & 1 & N
\end{array}
$$

and nef cone Nef $F$ spanned by $L, M$ and $N$. Let $X$ be a member of the nef linear system $|M+2 N|$. We have that $-K_{F}=L+M+3 N$ is ample, so $F$ is a Fano variety, and that $-K_{F}-\Lambda \sim L+N$ is nef but not ample on $F$. As is discussed in detail in the section on $\mathrm{MM}_{3-2}$, even though $-K_{F}-\Lambda$ is not ample on $F$, it becomes ample when restricted to $X$; thus the variety $X$ is Fano.

Write $p_{1}, p_{2}, p_{3} \in H^{\bullet}(F ; \mathbb{Z})$ for the first Chern classes of $L, M$ and $N$, respectively; these classes form a basis for $H^{2}(F ; \mathbb{Z})$. Identify the group ring $\mathbb{Q}\left[H_{2}(F ; \mathbb{Z})\right]$ with the polynomial ring $\mathbb{Q}\left[Q_{1}, Q_{2}, Q_{3}\right]$ via the $\mathbb{Q}$-linear map that sends the element $Q^{\beta} \in \mathbb{Q}\left[H_{2}(F ; \mathbb{Z})\right]$ to $Q_{1}^{\left\langle\beta, p_{1}\right\rangle} Q_{2}^{\left\langle\beta, p_{2}\right\rangle} Q_{3}^{\left\langle\beta, p_{3}\right\rangle}$. Theorem C. 1 gives

$$
\begin{aligned}
J_{F}(\tau)=e^{\tau / z} \sum_{(l, m, n) \in \mathbb{Z}^{3}} Q_{1}^{l} Q_{2}^{m} & Q_{3}^{n} e^{\langle\beta, \tau\rangle} \frac{\prod_{k=-\infty}^{0}\left(p_{1}+k z\right)^{2}}{\prod_{k=-\infty}^{l}\left(p_{1}+k z\right)^{2}} \frac{\prod_{k=-\infty}^{0}\left(p_{2}+k z\right)^{2}}{\prod_{k=-\infty}^{m}\left(p_{2}+k z\right)^{2}} \\
\times & \frac{\prod_{k=-\infty}^{0}\left(p_{3}+k z\right)^{2}}{\prod_{k=-\infty}^{n}\left(p_{3}+k z\right)^{2}} \frac{\prod_{k=-\infty}^{0}\left(p_{3}-p_{2}-p_{1}+k z\right)}{\prod_{k=-\infty}^{n-l-m}\left(p_{3}-p_{2}-p_{1}+k z\right)}
\end{aligned}
$$


and, since $p_{1}^{2}=p_{2}^{2}=p_{3}^{2}\left(p_{3}-p_{2}-p_{1}\right)=0$ in the cohomology of $F$, this reduces to

$$
\begin{array}{r}
J_{F}(\tau)=e^{\tau / z} \sum_{l, m, n \geq 0} \frac{Q_{1}^{l} Q_{2}^{m} Q_{3}^{n} e^{\langle\beta, \tau\rangle}}{\prod_{k=1}^{l}\left(p_{1}+k z\right)^{2} \prod_{k=1}^{m}\left(p_{2}+k z\right)^{2} \prod_{k=1}^{n}\left(p_{3}+k z\right)^{2}} \\
\times \frac{\prod_{k=-\infty}^{0}\left(p_{3}-p_{2}-p_{1}+k z\right)}{\prod_{k=-\infty}^{n-l-m}\left(p_{3}-p_{2}-p_{1}+k z\right)} .
\end{array}
$$

Thus

$$
\begin{array}{r}
I_{\boldsymbol{e}, E}(\tau)=e^{\tau / z} \sum_{l, m, n \geq 0} \frac{Q_{1}^{l} Q_{2}^{m} Q_{3}^{n} e^{\langle\beta, \tau\rangle} \prod_{k=1}^{m+2 n}\left(\lambda+p_{2}+2 p_{3}+k z\right)}{\prod_{k=1}^{l}\left(p_{1}+k z\right)^{2} \prod_{k=1}^{m}\left(p_{2}+k z\right)^{2} \prod_{k=1}^{n}\left(p_{3}+k z\right)^{2}} \\
\times \frac{\prod_{k=-\infty}^{0}\left(p_{3}-p_{2}-p_{1}+k z\right)}{\prod_{k=-\infty}^{n-l-m}\left(p_{3}-p_{2}-p_{1}+k z\right)} .
\end{array}
$$

We now apply Theorem D.3. Setting $\tau=0$, we find that

$$
I_{\boldsymbol{e}, E}(0)=A+B z^{-1}+O\left(z^{-2}\right),
$$

where

$$
\begin{aligned}
A=1 \quad \text { and } B & =\left(2 Q_{3}+6 Q_{2} Q_{3}\right) 1+\left(p_{3}-p_{2}-p_{1}\right) \sum_{m>0} \frac{(-1)^{m-1} Q_{2}^{m}}{m} \\
& =\left(2 Q_{3}+6 Q_{2} Q_{3}\right) 1+\left(p_{3}-p_{2}-p_{1}\right) \log \left(1+Q_{2}\right) .
\end{aligned}
$$

Thus

$$
J_{\boldsymbol{e}, E}(B)=I_{\boldsymbol{e}, E}(0)
$$

The string equation gives

$$
J_{\boldsymbol{e}, E}(c 1+\tau)=e^{c / z} J_{\boldsymbol{e}, E}(\tau),
$$

so

$$
J_{\boldsymbol{e}, E}\left(\left(p_{3}-p_{2}-p_{1}\right) \log \left(1+Q_{2}\right)\right)=e^{-\left(2 Q_{3}+6 Q_{2} Q_{3}\right) / z} I_{\boldsymbol{e}, E}(0) .
$$

The twisted $J$-function satisfies

$$
\begin{aligned}
J_{\boldsymbol{e}, E}\left(t_{1} p_{1}+t_{2} p_{2}\right. & \left.+t_{3} p_{3}\right) \\
& =e^{\left(t_{1} p_{1}+t_{2} p_{2}+t_{3} p_{3}\right) / z}\left(1+\sum_{l, m, n \geq 0} Q_{1}^{l} Q_{2}^{m} Q_{3}^{n} e^{l t_{1}} e^{m t_{2}} e^{n t_{3}} c_{l, m, n}\right)
\end{aligned}
$$

for classes $c_{l, m, n} \in H^{\bullet}(F ; \mathbb{Q}[\lambda]) \llbracket z^{-1} \rrbracket$ that do not depend on $t_{1}, t_{2}$ or $t_{3}$. So, substituting $t_{1}=t_{2}=-\log \left(1+Q_{2}\right)$ and $t_{3}=\log \left(1+Q_{2}\right)$, we see that

$$
\begin{aligned}
& J_{\boldsymbol{e}, E}\left(\left(p_{3}-p_{2}-p_{1}\right) \log \left(1+Q_{2}\right)\right) \\
& \quad=e^{\left(p_{3}-p_{2}-p_{1}\right) \log \left(1+Q_{2}\right) / z}\left[J_{\boldsymbol{e}, E}(0)\right]_{Q_{1}=\frac{Q_{1}}{1+Q_{2}}, Q_{2}=\frac{Q_{2}}{1+Q_{2}}, Q_{3}=Q_{3}\left(1+Q_{2}\right)}
\end{aligned}
$$


The change of variables

$$
Q_{1}=\frac{Q_{1}}{1+Q_{2}}, \quad Q_{2}=\frac{Q_{2}}{1+Q_{2}}, \quad Q_{3}=Q_{3}\left(1+Q_{2}\right)
$$

is called the mirror map; the inverse change of variables is

$$
Q_{1}=\frac{Q_{1}}{1-Q_{2}}, \quad Q_{2}=\frac{Q_{2}}{1-Q_{2}}, \quad Q_{3}=Q_{3}\left(1-Q_{2}\right),
$$

and so

$$
\begin{aligned}
J_{\boldsymbol{e}, E}(0)= & {\left[e^{-\left(p_{3}-p_{2}-p_{1}\right) \log \left(1+Q_{2}\right) / z}\right.} \\
& \left.\quad \times J_{\boldsymbol{e}, E}\left(\left(p_{3}-p_{2}-p_{1}\right) \log \left(1+Q_{2}\right)\right)\right]_{Q_{1}}=\frac{Q_{1}}{1-Q_{2}}, Q_{2}=\frac{Q_{2}}{1-Q_{2}}, Q_{3}=Q_{3}\left(1-Q_{2}\right) \\
= & e^{\left(p_{3}-p_{2}-p_{1}\right) \log \left(1-Q_{2}\right) / z} \\
& \quad \times\left[e^{-\left(2 Q_{3}+6 Q_{2} Q_{3}\right) / z} I_{\boldsymbol{e}, E}(0)\right]_{Q_{1}}=\frac{Q_{1}}{1-Q_{2}}, Q_{2}=\frac{Q_{2}}{1-Q_{2}}, Q_{3}=Q_{3}\left(1-Q_{2}\right)^{\circ}
\end{aligned}
$$

Taking the non-equivariant limit yields

$$
\begin{aligned}
& J_{F, X}(0)=e^{\left(p_{3}-p_{2}-p_{1}\right) \log \left(1-Q_{2}\right) / z} e^{-2 Q_{3}-4 Q_{2} Q_{3}} \\
& \times \sum_{l, m, n \geq 0} \frac{Q_{1}^{l} Q_{2}^{m} Q_{3}^{n}\left(1-Q_{2}\right)^{n-l-m} \prod_{k=1}^{m+2 n}\left(p_{2}+2 p_{3}+k z\right)}{\prod_{k=1}^{l}\left(p_{1}+k z\right)^{2} \prod_{k=1}^{m}\left(p_{2}+k z\right)^{2} \prod_{k=1}^{n}\left(p_{3}+k z\right)^{2}} \\
& \times \frac{\prod_{k=-\infty}^{0}\left(p_{3}-p_{2}-p_{1}+k z\right)}{\prod_{k=-\infty}^{n-l-m}\left(p_{3}-p_{2}-p_{1}+k z\right)} .
\end{aligned}
$$

Recall that the quantum period $G_{X}$ is obtained from the component of $J_{X}(0)$ along the unit class $1 \in H^{\bullet}(X ; \mathbb{Q})$ by setting $z=1$ and $Q^{\beta}=t^{\left\langle\beta,-K_{X}\right\rangle}$. Consider (8). To obtain $G_{X}$, therefore, we need to extract the component of $J_{F, X}(0)$ along the unit class $1 \in H^{\bullet}(F ; \mathbb{Q})$, set $z=1, Q_{1}=t, Q_{2}=1$ and $Q_{3}=t$. This gives

$$
G_{X}(t)=e^{-6 t} \sum_{l=0}^{\infty} \sum_{m=0}^{\infty} t^{2 l+m} \frac{(2 l+3 m) !}{(l !)^{2}(m !)^{2}((l+m) !)^{2}} .
$$

Regularizing gives

$$
\begin{aligned}
\widehat{G}_{X}(t)=1+58 t^{2}+600 t^{3} & +13182 t^{4}+247440 t^{5}+5212300 t^{6} \\
+ & 111835920 t^{7}+2480747710 t^{8}+56184565920 t^{9}+\cdots .
\end{aligned}
$$




\section{D.2 Weighted projective complete intersections}

We will need also an analogue of Corollary D.5 when the ambient space is weighted projective space, regarded as a smooth toric Deligne-Mumford stack rather than as a singular variety.

Proposition D.9 Let $Y$ be the weighted projective space $\mathbb{P}\left(w_{0}, \ldots, w_{n}\right)$, let $X$ be a smooth Fano variety given as a complete intersection in $Y$ defined by a section of $E=\mathcal{O}\left(d_{1}\right) \oplus \cdots \oplus \mathcal{O}\left(d_{m}\right)$, and let $-k=w_{0}+\cdots+w_{n}-d_{1}-\cdots-d_{m}$. Suppose that each $d_{i}$ is a positive integer, that $-k>0$ and that

$$
w_{i} \text { divides } d_{j} \text { for all } i \text { and } j \text { such that } 0 \leq i \leq n \text { and } 1 \leq j \leq m .
$$

Then the quantum period of $X$ is

$$
G_{X}(t)=e^{-c t} \sum_{d \geq 0} t^{-k d} \frac{\prod_{j=1}^{m}\left(d d_{j}\right) !}{\prod_{i=1}^{n}\left(d w_{i}\right) !},
$$

where $c$ is the unique rational number such that the right-hand side has the form $1+O\left(t^{2}\right)$.

Proof This follows immediately from [17, Corollary 1.9]. Corollary 1.9 as stated there is false, however, because it omits the divisibility hypothesis (10). This hypothesis ensures that the bundle $E$ is convex and hence ensures both (a) that the twisted $J_{-}$ function denoted by $J^{\text {tw }}$ in [13, Corollary 5.1] admits a non-equivariant limit $J_{Y, X}$ and (b) that this non-equivariant limit satisfies (8); see [15, Section 5]. Both (a) and (b) are used implicitly in the proof of [17, Corollary 1.9]. Under the additional divisibility assumption (10), however, the proof of [17, Corollary 1.9] goes through. This proves the proposition.

\section{E Geometric constructions}

Lemma E.1 Let $G$ be a nonsingular algebraic variety, let $V^{n+1}$ and $W^{n}$ be locally free sheaves on $G$ of ranks $n+1$ and $n$, respectively, and let $f: V \rightarrow W$ be a homomorphism of sheaves. Denote by $\pi: \mathbb{P}(V) \rightarrow G$ the projective space bundle of lines in $V$, so that there is a tautological exact sequence

$$
0 \rightarrow S \rightarrow \pi^{\star} V \rightarrow Q \rightarrow 0
$$

with $S^{\star}:=\mathcal{O}(1)$. Recall that elements of $V^{\star}$, being linear functions on $V$, define canonical sections of the line bundle $\mathcal{O}(1)$ on $\mathbb{P}(V)$ and that the corresponding homomorphism $\pi^{\star} V^{\star} \rightarrow \mathcal{O}(1)$ induces an isomorphism $V^{\star} \cong \pi_{\star} \mathcal{O}(1)$. The section 
$f \in \operatorname{Hom}_{G}(V, W)$ determines a section $\tilde{f} \in H^{0}\left(\mathbb{P}(V), \pi^{\star} W \otimes \mathcal{O}(1)\right)$ by means of the canonical identifications

$\operatorname{Hom}_{G}(V, W)=H^{0}\left(G, W \otimes V^{\star}\right)=H^{0}\left(G, W \otimes \pi_{\star} \mathcal{O}(1)\right)=H^{0}\left(\mathbb{P}(V), \pi^{\star} W \otimes \mathcal{O}(1)\right)$.

Let $F=Z(\tilde{f}) \subset \mathbb{P}(V)$ be the subscheme of $\mathbb{P}(V)$ where $\tilde{f}$ vanishes. Denote by $Z \subset G$ the subscheme where $f$ drops rank, that is, the ideal of $Z$ is the ideal defined by the $n+1$ minors of size $n$ of $f$. Assume (a) that $f$ has generically maximal rank; (b) that it drops rank in codimension 2 (this is the expected codimension); and (c) that $Z$ is nonsingular. ${ }^{7}$ Then $F$ is the blow-up of $G$ along $Z$.

Proof The statement is local on $G$, so fix a point $P \in Z \subset G$ and a Zariski open neighbourhood $P \in U=\operatorname{Spec} A$ with trivializations $\left.V\right|_{U}=A^{n+1}$ and $\left.W\right|_{U}=A^{n}$. The morphism $\left.f\right|_{U}$ is given by an $n \times(n+1)$ matrix $M$ with entries in $A$. Because $Z$ is nonsingular, at least one of the $(n-1) \times(n-1)$ minors of $A$ is non-zero at $P$ and then, after changing trivializations and shrinking $U$ if necessary, we may assume that

$$
M=\left(\begin{array}{cccccc}
1 & 0 & \cdots & 0 & 0 & 0 \\
0 & 1 & \cdots & 0 & 0 & 0 \\
\vdots & \vdots & \ddots & \vdots & \vdots & \vdots \\
0 & 0 & \cdots & 1 & 0 & 0 \\
0 & 0 & \cdots & 0 & x & y
\end{array}\right) .
$$

It is clear that the ideal generated by the $n \times n$ minors of $M$ is the ideal generated by the two rightmost minors $x$ and $y$ (and, since $Z$ is nonsingular, $x$ and $y$ form part of a regular system of parameters at $P$ ). Denoting by $x_{0}, \ldots, x_{n}$ the dual basis of $V^{\star},\left.F\right|_{U}=F \cap \pi^{-1}(U) \subset \mathbb{P}\left(\left.V\right|_{U}\right) \cong U \times \mathbb{P}^{n}$ is given by the $n$ equations in $n+1$ variables

$$
M \cdot\left(\begin{array}{c}
x_{0} \\
\vdots \\
x_{n}
\end{array}\right)=0 .
$$

The first $n-1$ equations just say $x_{0}=\cdots=x_{n-2}=0$, while the last equation states that $\left.F\right|_{U}$ is the variety $\left(x x_{n-1}+y x_{n}=0\right) \subset U \times \mathbb{P}_{x_{n-1}, x_{n}}^{1}$, that is, $F$ is the blow-up of $Z \subset G$.

We will need the following well-known construction:

Lemma E.2 Let $G$ be a complex Lie group acting on a space $A, X=A / / G$ a geometric quotient and $\rho: G \rightarrow \mathrm{GL}_{r}(\mathbb{C})$ a complex representation. Then:

\footnotetext{
${ }^{7}$ The last assumption (c) is probably not necessary.
} 
(1) $\rho$ naturally induces a vector bundle $E=E(\rho)$ on $X$. Explicitly, $E(\rho)=$ $\left(A \times \mathbb{C}^{r}\right) / / G$, where $G$ acts as

$$
g:(a, v) \mapsto(g a, \rho(g) v) .
$$

(2) Let $F=\mathbb{P}(E)$ be the bundle of 1-dimensional subspaces of the vector bundle in (1). Then $F=\left(A \times \mathbb{C}^{r}\right) / /\left(G \times \mathbb{C}^{\times}\right)$, where $G$ acts as in (1), and $\mathbb{C}^{\times}$acts trivially on the first factor and by rescaling on the second factor.

(3) Let $F=\mathbb{P}(E)$ be as in (2). The tautological line bundle $\mathcal{O}(-1)$ on $F$ is induced as in (1) by the 1-dimensional representation of $G \times \mathbb{C}^{\times}$that is trivial on the first factor and standard on the second.

We will also need to know how to compute the quantum period of a product in terms of the quantum periods of the factors.

Proposition E.3 (The small $J$-function of a product) Let $X$ and $Y$ be smooth projective varieties over $\mathbb{C}$. Recall that there is a canonical isomorphism $H^{\bullet}(X \times Y ; \mathbb{Q}) \cong$ $H^{\bullet}(X ; \mathbb{Q}) \otimes H^{\bullet}(Y ; \mathbb{Q})$ and that $\Lambda_{X \times Y}$ is a completion of $\Lambda_{X} \otimes \Lambda_{Y}$. Let $\tau_{X} \in H^{2}(X)$ and $\tau_{Y} \in H^{2}(Y)$. Then

$$
J_{X \times Y}\left(\tau_{X} \otimes 1+1 \otimes \tau_{Y}\right)=J_{X}\left(\tau_{X}\right) \otimes J_{Y}\left(\tau_{Y}\right) .
$$

\section{Proof Combine}

- the differential equations [16, Equation 16] that characterize the $J$-function;

- the fact that the small quantum product $*_{\tau}, \tau \in H^{2}$, is uniquely determined by three-point Gromov-Witten invariants and the divisor equation;

- the product formula for Gromov-Witten invariants $[42 ; 6]$ relating three-point Gromov-Witten invariants of $X \times Y$ to those of $X$ and of $Y$.

Corollary E.4 (The quantum period of a product) Let $X$ and $Y$ be smooth projective varieties over $\mathbb{C}$. Then

$$
G_{X \times Y}(t)=G_{X}(t) G_{Y}(t)
$$

\section{Notation for Grassmannians}

We denote by $\mathrm{Gr}=\operatorname{Gr}(r, n)$ the manifold of $r$-dimensional vector subspaces of $\mathbb{C}^{n}$. Notation for the universal sequence is

$$
0 \rightarrow S \rightarrow \mathbb{C}^{n} \rightarrow Q \rightarrow 0,
$$


where $S$ is the rank- $r$ universal bundle of subspaces and $Q$ is the rank- $n-r$ universal bundle of quotients. If $\lambda=\left(\lambda_{1} \geq \lambda_{2} \geq \cdots\right)$ is a partition or Young diagram, we denote by $Z_{\lambda} \subset \mathrm{Gr}$ the Schubert variety corresponding to $\lambda$ and by $\sigma_{\lambda} \in H^{\bullet}(\mathrm{Gr} ; \mathbb{Z})$ its class in cohomology. It is well known that $c_{i}\left(S^{\star}\right)=\sigma_{1^{i}}$ and $c_{i}(Q)=\sigma_{i}$ for $i=1,2,3, \ldots$

We will need:

- The Pieri formula: if $\lambda$ is a partition and $k \geq 0$ an integer then

$$
\sigma_{\lambda} \cdot \sigma_{k}=\sum_{\begin{array}{c}
\mu \geq \lambda \\
\text { adds } k \text { boxes, } \\
\text { no two in a column }
\end{array}} \sigma_{\mu} .
$$

- The following elementary facts for $\operatorname{Gr}(2,5)$ :

- The Plücker embedding sends the Schubert variety

$$
Z_{2}=\left\{W \mid W \cap\left\langle e_{0}, e_{1}\right\rangle \neq\{0\}\right\}
$$

to the subset of $\mathbb{P}^{9}$ defined by the equations $z_{23}=z_{24}=z_{34}=0$ and

$$
\operatorname{rk}\left(\begin{array}{ccc}
z_{02} & z_{03} & z_{04} \\
z_{12} & z_{13} & z_{14}
\end{array}\right)<2
$$

- The Plücker embedding sends the Schubert variety

$$
Z_{1,1}=\left\{W \mid W \subset\left\langle e_{0}, e_{1}, e_{2}, e_{3}\right\rangle\right\} \cong \operatorname{Gr}(2,4)
$$

to a nonsingular quadric.

\section{F The abelian/non-abelian correspondence}

Our other main tool for computing quantum periods is the abelian/non-abelian correspondence of Ciocan-Fontanine, Kim and Sabbah [9]. This expresses genus-zero Gromov-Witten invariants (or twisted Gromov-Witten invariants) of $X / / G$, where $G$ is a complex reductive Lie group and $X$ is a smooth projective variety, in terms of genus-zero Gromov-Witten invariants (or twisted Gromov-Witten invariants) of $X / / T$, where $T$ is a maximal torus in $G$. The computations for $X / / T$ are typically much easier - the methods of Sections C-D often apply, for example — so the abelian/nonabelian correspondence is a powerful tool for calculations. Ten of the seventeen smooth Fano 3-folds of Picard rank 1 are toric varieties or toric complete intersections and thus can be treated using the methods of Sections C-D; the following theorem allows a uniform treatment of the remaining seven cases. 
Theorem F.1 Let Gr denote the Grassmannian $\operatorname{Gr}(r, n)$ of $r$-dimensional subspaces of $\mathbb{C}^{n}$, let $S \rightarrow$ Gr denote the universal bundle of subspaces, and let $E \rightarrow$ Gr denote the vector bundle

$E=\left(\operatorname{det} S^{\star}\right)^{\oplus a} \oplus\left(\operatorname{det} S^{\star} \otimes \operatorname{det} S^{\star}\right)^{\oplus b} \oplus\left(S^{\star} \otimes \operatorname{det} S^{\star}\right)^{\oplus c}$

$\oplus\left(S \otimes \operatorname{det} S^{\star}\right)^{\oplus d} \oplus\left(\bigwedge^{2} S^{\star}\right)^{\oplus e}$.

Let $X$ be the subvariety of $\mathrm{Gr}$ cut out by a generic section of $E$ and suppose that

$$
k=a+2 b+(r+1) c+(r-1) d+(r-1) e-n
$$

is strictly negative. Consider the cohomology algebra $H^{\bullet}\left(\left(\mathbb{P}^{n-1}\right)^{r} ; \mathbb{Q}\right)$. Let $p_{i}$ in $H^{2}\left(\left(\mathbb{P}^{n-1}\right)^{r}\right)$ for $1 \leq i \leq r$ denote the first Chern class of $\pi_{i}^{\star} \mathcal{O}(1)$, where $\pi_{i}:\left(\mathbb{P}^{n-1}\right)^{r} \rightarrow \mathbb{P}^{n-1}$ is projection to the $i^{\text {th }}$ factor of the product. Let $p_{1 \cdots r}=$ $p_{1}+\cdots+p_{r}$ and, for $\left(l_{1}, \ldots, l_{r}\right) \in \mathbb{Z}^{r}$, let $|l|=l_{1}+\cdots+l_{r}$. Let

$\Gamma_{l_{1}, \ldots, l_{r}}=\left(\prod_{k=1}^{|l|}\left(p_{1 \ldots r}+k\right)\right)^{a}\left(\prod_{k=1}^{2|l|}\left(2 p_{1 \cdots r}+k\right)\right)^{b}\left(\prod_{j=1}^{r} \prod_{k=1}^{|l|+l_{j}}\left(p_{1 \cdots r}+p_{j}+k\right)\right)^{c}$

$$
\times\left(\prod_{j=1}^{r} \prod_{k=1}^{|l|-l_{j}}\left(p_{1 \cdots r}-p_{j}+k\right)\right)^{d}\left(\prod_{i=1}^{r-1} \prod_{j=i+1}^{r} \prod_{k=1}^{l_{i}+l_{j}}\left(p_{i}+p_{j}+k\right)\right)^{e}
$$

and let

$$
\Omega=\prod_{i=1}^{r-1} \prod_{j=i+1}^{r}\left(p_{j}-p_{i}\right)
$$

The element

$$
\sum_{l_{1}=0}^{\infty} \cdots \sum_{l_{r}=0}^{\infty} \frac{(-1)^{|l|(r-1)} t^{-k|l|} \Gamma_{l_{1}, \ldots, l_{r}}}{\prod_{j=1}^{r} \prod_{k=1}^{l_{r}}\left(p_{j}+k\right)^{n}} \prod_{i=1}^{r}\left(p_{j=i+1}-p_{i}+\left(l_{j}-l_{i}\right)\right)
$$

of $H^{\bullet}\left(\left(\mathbb{P}^{n-1}\right)^{r} ; \mathbb{Q}\right) \otimes \mathbb{Q} \llbracket t \rrbracket$ is divisible by $\Omega$. Let $I_{\mathrm{tw}}(t)$ be the scalar-valued function obtained by dividing (11) by $\Omega$ and taking the component along $H^{0}\left(\left(\mathbb{P}^{n-1}\right)^{r} ; \mathbb{Q}\right)$. Then the quantum period $G_{X}$ of $X$ satisfies

$$
G_{X}(t)=e^{\alpha t} I_{\mathrm{tw}}(t),
$$

where $\alpha$ is the unique rational number such that the right-hand side has the form $1+O\left(t^{2}\right)$.

Proof The expression (11) is divisible by $\Omega$ because it is totally antisymmetric in $p_{1}, \ldots, p_{r}$. We know a priori that $G_{X}(t)=1+O\left(t^{2}\right)$, so if there exists $\alpha \in \mathbb{Q}$ such that $G_{X}(t)=e^{\alpha t} I_{\mathrm{tw}}(t)$ then this $\alpha$ is uniquely determined by the condition 
$e^{\alpha t} I_{\mathrm{tw}}(t)=1+O\left(t^{2}\right)$. For the rest we use the abelian/non-abelian correspondence. Consider the situation as in [9, Section 3.1] with

- the space that is denoted by $X$ in [9] set equal to $A=\mathbb{C}^{r n}$, regarded as the space of $r \times n$ matrices;

- $G=\mathrm{GL}_{r}(\mathbb{C})$, acting on $A$ by left-multiplication;

- $T=\left(\mathbb{C}^{\times}\right)^{r}$, the diagonal torus in $G$;

- the group that is denoted by $S$ in [9] set equal to the trivial group;

- $\mathcal{V}$ equal to the representation $\left(\operatorname{det} V_{\text {std }}\right)^{\oplus a} \oplus\left(\operatorname{det} V_{\text {std }} \otimes \operatorname{det} V_{\text {std }}\right)^{\oplus b} \oplus\left(V_{\text {std }} \otimes \operatorname{det} V_{\text {std }}\right)^{\oplus c}$

$$
\oplus\left(V_{\text {std }}^{\star} \otimes \operatorname{det} V_{\text {std }}\right)^{\oplus d} \oplus\left(\bigwedge^{2} V_{\text {std }}\right)^{\oplus e}
$$

where $V_{\text {std }}$ is the standard representation of $G$.

Then $A / / G$ is the Grassmannian $\mathrm{Gr}=\operatorname{Gr}(r, n)$ and $A / / T$ is $\left(\mathbb{P}^{n-1}\right)^{r}$. The Weyl group $W=S_{r}$ permutes the $r$ factors of the product $\left(\mathbb{P}^{n-1}\right)^{r}$. The representation $\mathcal{V}$ induces the vector bundle $\mathcal{V}_{G}=E$ over $A / / G=\mathrm{Gr}$ and the representation $\mathcal{V}$ induces the vector bundle

$$
\begin{aligned}
\mathcal{V}_{T}= & (\mathcal{O}(1,1, \ldots, 1))^{\oplus a} \oplus(\mathcal{O}(2,2, \ldots, 2))^{\oplus b} \oplus\left(\bigoplus_{j=1}^{r} \mathcal{O}(1,1, \ldots, 1) \otimes \pi_{j}^{\star} \mathcal{O}(1)\right)^{\oplus c} \\
& \oplus\left(\bigoplus_{j=1}^{r} \mathcal{O}(1,1, \ldots, 1) \otimes \pi_{j}^{\star} \mathcal{O}(-1)\right)^{\oplus d} \oplus\left(\bigoplus_{i=1}^{r-1} \bigoplus_{j=i+1}^{r} \pi_{i}^{\star} \mathcal{O}(1) \otimes \pi_{j}^{\star} \mathcal{O}(1)\right)^{\oplus e}
\end{aligned}
$$

over $A / / T=\left(\mathbb{P}^{n-1}\right)^{r}$.

We fix a lift of $H^{\bullet}(A / / G ; \mathbb{Q})$ to $H^{\bullet}(A / / T, \mathbb{Q})^{W}$ in the sense of $[9$, Section 3]; there are many possible choices for such a lift and the precise choice made will be unimportant in what follows. The lift allows us to regard $H^{\bullet}(A / / G ; \mathbb{Q})$ as a subspace of $H^{\bullet}(A / / T, \mathbb{Q})^{W}$, which maps isomorphically to the Weyl-anti-invariant part $H^{\bullet}(A / / T, \mathbb{Q})^{a}$ of $H^{\bullet}(A / / T, \mathbb{Q})$ via

$$
H^{\bullet}(A / / T, \mathbb{Q})^{W} \stackrel{\cup \Omega}{\longrightarrow} H^{\bullet}(A / / T, \mathbb{Q})^{a} .
$$

We compute the quantum period of $X$ by computing the $J$-function of $\mathrm{Gr}=A / / G$ twisted [16] by the Euler class and the bundle $\mathcal{V}_{G}$, using the abelian/non-abelian correspondence [9].

We begin by computing the $J$-function of $A / / T$ twisted by the Euler class and the bundle $\mathcal{V}_{T}$. As in Section D.1, and as in [9], consider the bundles $\mathcal{V}_{T}$ and $\mathcal{V}_{G}$ 
equipped with the canonical $\mathbb{C}^{\times}$-action that rotates fibres and acts trivially on the base. We will compute the twisted $J$-function $J_{\boldsymbol{e}, \mathcal{V}_{T}}$ of $A / / T$ using the quantum Lefschetz theorem; $J_{\boldsymbol{e}, \mathcal{V}_{T}}$ was defined in (6) above and is the restriction to the locus $\tau \in H^{0}(A / / T) \oplus H^{2}(A / / T)$ of what was denoted by $J_{\mathcal{V}_{T} \times \mathbb{C}^{\times}}(\tau)$ in [9]. The toric variety $A / / T$ is Fano, and Theorem C.1 gives

$$
J_{A / / T}(\tau)=e^{\tau / z} \sum_{l_{1}=0}^{\infty} \cdots \sum_{l_{r}=0}^{\infty} \frac{Q_{1}^{l_{1}} \cdots Q_{r}^{l_{r}} e^{l_{1} \tau_{1}} \cdots e^{l_{r} \tau_{r}}}{\prod_{j=1}^{r} \prod_{k=1}^{l_{j}}\left(p_{j}+k z\right)^{n}},
$$

where $\tau=\tau_{1} p_{1}+\cdots+\tau_{r} p_{r}$ and we have identified the group ring $\mathbb{Q}\left[H_{2}(A / / T ; \mathbb{Z})\right]$ with $\mathbb{Q}\left[Q_{1}, \ldots, Q_{r}\right]$ via the $\mathbb{Q}$-linear map that sends $Q^{\beta}$ to $Q_{1}^{\left\langle\beta, p_{1}\right\rangle} \cdots Q_{r}^{\left\langle\beta, p_{r}\right\rangle}$. Each line bundle summand in $\mathcal{V}_{T}$ is nef, and the condition $k<0$ ensures that $c_{1}(A / / T)-c_{1}\left(\mathcal{V}_{T}\right)$ is ample, so Theorem D.3 gives

$$
\begin{aligned}
& J_{\boldsymbol{e}, \mathcal{V}_{T}}(\tau)=e^{c\left(Q_{1} e^{\tau_{1}+\cdots+Q_{r}} e^{\tau_{r}}\right) / z} e^{\tau / z} \\
& \times \sum_{l_{1}=0}^{\infty} \cdots \sum_{l_{r}=0}^{\infty} \frac{Q_{1}^{l_{1}} \cdots Q_{r}^{l_{r}} e^{l_{1} \tau_{1}} \cdots e^{l_{r} \tau_{r}} \Gamma_{l_{1}, \ldots, l_{r}}(\lambda, z)}{\prod_{j=1}^{r} \prod_{k=1}^{l_{j}}\left(p_{j}+k z\right)^{n}}
\end{aligned}
$$

for some rational number $c$, where

$$
\begin{aligned}
\Gamma_{l_{1}, \ldots, l_{r}}(\lambda, z)=( & \left.\prod_{k=1}^{|l|}\left(\lambda+p_{1 \ldots r}+k z\right)\right)^{a}\left(\prod_{k=1}^{2|l|}\left(\lambda+2 p_{1 \ldots r}+k z\right)\right)^{b} \\
& \times\left(\prod_{j=1}^{r} \prod_{k=1}^{|l|+l_{j}}\left(\lambda+p_{1} \ldots r+p_{j}+k z\right)\right)^{c}\left(\prod_{j=1}^{r} \prod_{k=1}^{|l|-l_{j}}\left(\lambda+p_{1 \ldots r}-p_{j}+k z\right)\right)^{d} \\
& \times\left(\prod_{i=1}^{r-1} \prod_{j=i+1}^{r} \prod_{k=1}^{l_{i}+l_{j}}\left(\lambda+p_{i}+p_{j}+k z\right)\right)^{e} .
\end{aligned}
$$

The prefactor $e^{c\left(Q_{1} e^{\tau} 1+\cdots+Q_{r} e^{\tau_{r}}\right) / z}$ in (12) comes from the prefactor $e^{-C(\tau) / z}$ in Theorem D.3.

Consider now $A / / G$ and a point $t \in H^{\bullet}(A / / G)$. In [9, Section 6.1] the authors consider the lift $\tilde{J}_{\mathcal{V}_{G}}^{S \times \mathbb{C}^{\times}}(t)$ of their twisted $J$-function $J_{\mathcal{V}_{G}}^{S \times \mathbb{C}^{\times}}(t)$ determined by a choice of lift

$$
H^{\bullet}(A / / G ; \mathbb{Q}) \rightarrow H^{\bullet}(A / / T, \mathbb{Q})^{W} .
$$

We restrict to the locus $t \in H^{0}(A / / G ; \mathbb{Q}) \oplus H^{2}(A / / G ; \mathbb{Q})$, considering the lift

$$
\tilde{J}_{\boldsymbol{e}, \mathcal{V}_{G}}(t):=\tilde{J}_{\mathcal{V}_{G} \times \mathbb{C}^{\times}}(t), \quad t \in H^{0}(A / / G ; \mathbb{Q}) \oplus H^{2}(A / / G ; \mathbb{Q}),
$$


of our twisted $J$-function $J_{e}, \mathcal{V}_{G}$ determined by our choice of lift

$$
H^{\bullet}(A / / G ; \mathbb{Q}) \rightarrow H^{\bullet}(A / / T, \mathbb{Q})^{W} .
$$

Let $p$ be the ample generator for $H^{2}(A / / G ; \mathbb{Z}) \cong \mathbb{Z}$ and identify the group ring $\mathbb{Q}\left[H_{2}(A / / G ; \mathbb{Z})\right]$ with $\mathbb{Q}[q]$ via the $\mathbb{Q}$-linear map which sends $Q^{\beta}$ to $q^{\langle\beta, p\rangle}$. Theorems 4.1.1 and 6.1.2 in [9] imply that

$$
\tilde{J}_{\boldsymbol{e}, \mathcal{V}_{G}}(\theta(t)) \cup \Omega=\left[\left(\prod_{i=1}^{r-1} \prod_{j=i+1}^{r}\left(z \frac{\partial}{\partial \tau_{j}}-z \frac{\partial}{\partial \tau_{i}}\right)\right) J_{\boldsymbol{e}, \mathcal{V}_{T}}(\tau)\right]_{\tau=t, Q_{1}=\cdots=Q_{r}=(-1)^{r-1} q}
$$

for some ${ }^{8}$ function $\theta: H^{2}(A / / G ; \mathbb{Q}) \rightarrow H^{\bullet}\left(A / / G ; \Lambda_{A / / G}\right)$ such that $\theta(0)=c^{\prime} q$ is in $H^{0}(A / / G ; \mathbb{Q}) \otimes \Lambda_{A / / G}$. Setting $t=0$ gives

$\tilde{J}_{\boldsymbol{e}, \mathcal{V}_{G}}\left(c^{\prime} q\right) \cup \Omega$

$$
=e^{ \pm c r q / z} \sum_{l_{1}=0}^{\infty} \cdots \sum_{l_{r}=0}^{\infty} \frac{(-1)^{|l|(r-1)} q^{|l|} \Gamma_{l_{1}, \ldots, l_{r}}(\lambda, z)}{\prod_{j=1}^{r} \prod_{k=1}^{l_{j}}\left(p_{j}+k z\right)^{n}} \prod_{i=1}^{r-1} \prod_{j=i+1}^{r}\left(p_{j}-p_{i}+\left(l_{j}-l_{i}\right) z\right) .
$$

The string equation gives

$$
\tilde{J}_{\boldsymbol{e}, \mathcal{V}_{G}}\left(c^{\prime} q\right)=e^{c^{\prime} q / z} \tilde{J}_{\boldsymbol{e}, \mathcal{V}_{G}}(0)
$$

and therefore

$$
\begin{aligned}
& \tilde{J}_{\boldsymbol{e}, \mathcal{V}_{G}}(0) \cup \Omega \\
& =e^{\alpha q / z} \sum_{l_{1}=0}^{\infty} \cdots \sum_{l_{r}=0}^{\infty} \frac{(-1)^{|l|(r-1)} q^{|l|} \Gamma_{l_{1}, \ldots, l_{r}}(\lambda, z)}{\prod_{j=1}^{r} \prod_{k=1}^{l_{j}}\left(p_{j}+k z\right)^{n}} \prod_{i=1}^{r-1} \prod_{j=i+1}^{r}\left(p_{j}-p_{i}+\left(l_{j}-l_{i}\right) z\right),
\end{aligned}
$$

where $\alpha=-c^{\prime} \pm c r$. Note that if $k<-1$ then $\alpha=0$, for in that case both $c$ and $c^{\prime}$ are zero. Note also that $\Gamma_{l_{1}, \ldots, l_{r}}(0,1)$ coincides with what was denoted $\Gamma_{l_{1}, \ldots, l_{r}}$ in the statement of the theorem.

We saw in Example D.8 how to extract the quantum period $G_{X}$ from the twisted $J$-function $J_{\boldsymbol{e}, \mathcal{V}_{G}}(0)$ : we take the non-equivariant limit $\lambda \rightarrow 0$, extract the component along the unit class $1 \in H^{\bullet}(A / / G ; \mathbb{Q})$, set $z=1$ and set $Q^{\beta}=t^{\left\langle\beta,-K_{X}\right\rangle}$. Thus we consider the right-hand side of (13), take the non-equivariant limit, extract the coefficient of $\Omega$, set $z=1$ and set $q=t^{-k}$. The theorem follows.

${ }^{8}$ The map $\theta$ here is the inverse to the map denoted by $\varphi$ in [9]; it is grading-preserving, where cohomology classes have their usual degree and $q$ has degree $-2 k$. Furthermore, $\theta$ is the identity map modulo $q$. It follows that $\theta(0)=c^{\prime} q \in H^{0}(A / / G ; \mathbb{Q}) \otimes \Lambda_{A / / G}$ for some $c^{\prime} \in \mathbb{Q}$ and that $c^{\prime}=0$ whenever $k<-1$. 


\section{G Fano manifolds of dimension 1 and 2}

As a warm-up exercise, and because we will need some of these results in the $3-$ dimensional calculation, we now compute the quantum periods for all Fano manifolds of dimension 1 and 2.

Example G.1 There is a unique Fano manifold of dimension 1: the projective line $\mathbb{P}^{1}$. This is the toric variety with weight data

\section{1}

and nef cone given by the non-negative half-line in $\mathbb{R}$. Corollary C.2 gives

$$
G_{\mathbb{P}^{1}}(t)=\sum_{d=0}^{\infty} \frac{t^{2 d}}{(d !)^{2}} .
$$

\section{Del Pezzo surfaces}

There are 10 deformation families of Fano manifolds of dimension 2: these are the del Pezzo surfaces. It is well known that, up to deformation,

- there is a unique smooth Fano surface of degree 9, being the projective plane $\mathbb{P}^{2}$;

- there are two smooth Fano surfaces of degree 8, being the Hirzebruch surface $\mathbb{F}_{1}$ and the product of projective lines $\mathbb{P}^{1} \times \mathbb{P}^{1}$;

- there is a unique deformation class of smooth Fano surfaces $S_{d}$ of degree $d$, $1 \leq d \leq 7$.

Given this, it is easy to see that the del Pezzo surfaces can be constructed, and their quantum periods calculated, as follows.

Example G.2 The del Pezzo surface $\mathbb{P}^{2}$ is the toric variety with weight data

$$
111
$$

and nef cone equal to the non-negative half-line. Corollary C.2 gives

$$
G_{\mathbb{P}^{2}}(t)=\sum_{d=0}^{\infty} \frac{t^{3 d}}{(d !)^{3}} .
$$

Example G.3 The del Pezzo surface $\mathbb{P}^{1} \times \mathbb{P}^{1}$ is the toric variety with weight data

$$
\begin{aligned}
& \begin{array}{lllll}
1 & 1 & 0 & 0 & L
\end{array} \\
& \begin{array}{llllll}
0 & 0 & 1 & 1 & M
\end{array}
\end{aligned}
$$


and nef cone equal to $\langle L, M\rangle$. (Here and henceforth, $\left\langle L_{1}, \ldots, L_{k}\right\rangle$ denotes the cone spanned by $L_{1}, \ldots, L_{k}$.) Corollary C.2 gives

$$
G_{\mathbb{P}^{1} \times \mathbb{P}^{1}}(t)=\sum_{l=0}^{\infty} \sum_{m=0}^{\infty} \frac{t^{2 l+2 m}}{(l !)^{2}(m !)^{2}} .
$$

Example G.4 The del Pezzo surface $\mathbb{F}_{1}$ is the toric variety with weight data

$$
\begin{array}{rrrrr}
1 & 1 & -1 & 0 & L \\
0 & 0 & 1 & 1 & M
\end{array}
$$

and nef cone equal to $\langle L, M\rangle$. Corollary C.2 gives

$$
G_{\mathbb{F}_{1}}(t)=\sum_{l=0}^{\infty} \sum_{m=l}^{\infty} \frac{t^{l+2 m}}{(l !)^{2}(m-l) ! m !} .
$$

Example G.5 The del Pezzo surface $S_{7}$ is the toric variety with weight data

$$
\begin{array}{rrrrrc}
1 & 0 & 1 & -1 & 0 & L \\
0 & 1 & 1 & 0 & -1 & M \\
0 & 0 & -1 & 1 & 1 & N
\end{array}
$$

and nef cone equal to $\langle L, M, N\rangle$. Corollary C.2 gives

$$
G_{S_{7}}(t)=\sum_{l=0}^{\infty} \sum_{m=0}^{\infty} \sum_{n=\max (l, m)}^{l+m} \frac{t^{l+m+n}}{l ! m !(l+m-n) !(n-l) !(n-m) !} .
$$

Example G.6 The del Pezzo surface $S_{6}$ is the toric variety with weight data

$$
\begin{array}{rrrrrrr}
1 & 0 & 0 & 0 & 1 & -1 & A \\
0 & 1 & 0 & 0 & 1 & 0 & B \\
0 & 0 & 1 & 0 & 0 & 1 & C \\
0 & 0 & 0 & 1 & -1 & 1 & D
\end{array}
$$

and nef cone equal to $\langle A+B, B+C, C+D, A+B+C, B+C+D\rangle$. Corollary C.2 gives

$$
G_{S_{6}}(t)=\sum_{a=0}^{\infty} \sum_{b=0}^{\infty} \sum_{c=0}^{\infty} \sum_{d=\max (a-c, 0)}^{a+b} \frac{t^{a+2 b+2 c+d}}{a ! b ! c ! d !(a+b-d) !(c+d-a) !} .
$$

Example G.7 The del Pezzo surface $S_{5}$ is a hypersurface of bidegree $(1,2)$ in $\mathbb{P}^{1} \times \mathbb{P}^{2}$. The ambient space $\mathbb{P}^{1} \times \mathbb{P}^{2}$ is the toric variety with weight data

$$
\begin{aligned}
& \begin{array}{lllllll}
1 & 1 & 0 & 0 & 0 & L
\end{array} \\
& \begin{array}{lllllll}
0 & 0 & 1 & 1 & 1 & M
\end{array}
\end{aligned}
$$


and nef cone equal to $\langle L, M\rangle$, and $S_{5}$ is a member of $|L+2 M|$ on $\mathbb{P}^{1} \times \mathbb{P}^{2}$. Corollary D.5 gives

$$
G_{S_{5}}(t)=e^{-3 t} \sum_{l=0}^{\infty} \sum_{m=0}^{\infty} t^{l+m} \frac{(l+2 m) !}{(l !)^{2}(m !)^{3}} .
$$

Example G.8 A complete intersection of type $(2,2)$ in $\mathbb{P}^{4}$ is a del Pezzo surface $S_{4}$. Proposition D.9 gives

$$
G_{S_{4}}(t)=e^{-4 t} \sum_{d=0}^{\infty} t^{d} \frac{(2 d) !(2 d) !}{(d !)^{5}} .
$$

Example G.9 A cubic surface in $\mathbb{P}^{3}$ is a del Pezzo surface $S_{3}$. Proposition D.9 gives

$$
G_{S_{3}}(t)=e^{-6 t} \sum_{d=0}^{\infty} t^{d} \frac{(3 d) !}{(d !)^{4}} .
$$

Example G.10 A quartic surface in $\mathbb{P}(1,1,1,2)$ is a del Pezzo surface $S_{2}$. Proposition D.9 gives

$$
G_{S_{2}}(t)=e^{-12 t} \sum_{d=0}^{\infty} t^{d} \frac{(4 d) !}{(d !)^{3}(2 d) !} .
$$

Example G.11 A sextic surface in $\mathbb{P}(1,1,2,3)$ is a del Pezzo surface $S_{1}$. Proposition D.9 gives

$$
G_{S_{1}}(t)=e^{-60 t} \sum_{d=0}^{\infty} t^{d} \frac{(6 d) !}{(d !)^{2}(2 d) !(3 d) !} .
$$

\section{H Notation for 3-dimensional Fano manifolds}

We fix notation for 3-dimensional Fano manifolds as follows:

- $\mathbb{P}^{3}$ denotes 3-dimensional complex projective space.

- $Q^{3}$ denotes a quadric hypersurface in $\mathbb{P}^{4}$.

- $\quad V_{k}$ denotes the 3-dimensional Fano manifold of Picard rank 1, Fano index 1 and degree $k$.

- $\quad B_{k}$ denotes the 3-dimensional Fano manifold of Picard rank 1, Fano index 2 and degree $8 k$. 
- $\mathrm{MM}_{\rho-k}$ denotes the $k^{\text {th }}$ entry in the Mori-Mukai list [53] of 3-dimensional Fano manifolds of Picard rank $\rho$, with the exception of the case $\rho=4$, where we place the $13^{\text {th }}$ entry in Mori and Mukai's rank-4 list [53, pages 48-49] in between the first and second elements of that list. This reordering ensures that, for each $\rho$, the sequence $\mathrm{MM}_{\rho-1}, \mathrm{MM}_{\rho-2}, \mathrm{MM}_{\rho-3}, \ldots$ is in order of increasing degree.

\section{The Fano manifold $\mathbb{P}^{3}$}

Iskovskikh classification This is case 1 in [37, Table 6.5].

Construction The Fano toric variety $X$ with weight data

\section{$\begin{array}{lllll}1 & 1 & 1 & 1 & L\end{array}$}

and Nef $X$ spanned by $L$.

The quantum period Corollary C.2 yields

and regularizing gives

$$
G_{X}(t)=\sum_{d=0}^{\infty} \frac{t^{4 d}}{(d !)^{4}}
$$

$$
\widehat{G}_{X}(t)=1+24 t^{4}+2520 t^{8}+369600 t^{12}+\cdots .
$$

Minkowski period sequence 1

\section{The Fano manifold $Q^{3}$}

Iskovskikh classification This is case 2 in [37, Table 6.5].

Construction A divisor $X$ of degree 2 on $F=\mathbb{P}^{4}$.

The quantum period The toric variety $F$ has weight data

$$
\begin{array}{llllll}
1 & 1 & 1 & 1 & 1 & L
\end{array}
$$

and Nef $F=\langle L\rangle$. We have:

- $F$ is a Fano variety.

- $\quad X \sim 2 L$ is ample.

- $-\left(K_{F}+X\right) \sim 3 L$ is ample. 
Corollary D.5 yields

$$
G_{X}(t)=\sum_{d=0}^{\infty} t^{3 d} \frac{(2 d) !}{(d !)^{5}}
$$

and regularizing gives

$$
\widehat{G}_{X}(t)=1+12 t^{3}+540 t^{6}+33600 t^{9}+2425500 t^{12}+\cdots .
$$

Minkowski period sequence 3

\section{The Fano manifold $B_{1}$}

Iskovskikh classification This is case 3 in [37, Table 6.5].

Construction A sextic hypersurface $X$ in $\mathbb{P}(1,1,1,2,3)$.

The quantum period Proposition D.9 yields

$$
G_{X}(t)=\sum_{d=0}^{\infty} t^{2 d} \frac{(6 d) !}{(d !)^{3}(2 d) !(3 d) !}
$$

and regularizing gives

$$
\begin{aligned}
\widehat{G}_{X}(t)=1+120 t^{2}+83160 t^{4}+81681600 t^{6}+93699005400 t^{8} \\
+117386113965120 t^{10}+\cdots .
\end{aligned}
$$

Minkowski period sequence None. Note that the anticanonical line bundle of $B_{1}$ is not very ample.

\section{The Fano manifold $B_{2}$}

Iskovskikh classification This is case 4 in [37, Table 6.5].

Construction A quartic hypersurface $X$ in $\mathbb{P}(1,1,1,1,2)$.

The quantum period Proposition D.9 yields

$$
G_{X}(t)=\sum_{d=0}^{\infty} t^{2 d} \frac{(4 d) !}{(d !)^{4}(2 d) !}
$$


and regularizing gives

$$
\widehat{G}_{X}(t)=1+24 t^{2}+2520 t^{4}+369600 t^{6}+63063000 t^{8}+11732745024 t^{10}+\cdots .
$$

Minkowski period sequence 140

\section{The Fano manifold $B_{3}$}

Iskovskikh classification This is case 5 in [37, Table 6.5].

Construction A divisor $X$ of degree 3 on $F=\mathbb{P}^{4}$.

The quantum period The toric variety $F$ has weight data

$$
\begin{array}{llllll}
1 & 1 & 1 & 1 & 1 & L
\end{array}
$$

and Nef $F=\langle L\rangle$. We have:

- $F$ is a Fano variety.

- $\quad X \sim 3 L$ is ample.

- $-\left(K_{F}+X\right) \sim 2 L$ is ample.

Corollary D.5 yields

$$
G_{X}(t)=\sum_{d=0}^{\infty} t^{2 d} \frac{(3 d) !}{(d !)^{5}}
$$

and regularizing gives

$$
\widehat{G}_{X}(t)=1+12 t^{2}+540 t^{4}+33600 t^{6}+2425500 t^{8}+190702512 t^{10}+\cdots .
$$

Minkowski period sequence 106

\section{The Fano manifold $B_{4}$}

Iskovskikh classification This is case 6 in [37, Table 6.5].

Construction A codimension- 2 complete intersection $X$ of type $(2 L) \cap(2 L)$ in the toric variety $F=\mathbb{P}^{5}$. 
The quantum period The toric variety $F$ has weight data

$$
\begin{array}{lllllll}
1 & 1 & 1 & 1 & 1 & 1 & L
\end{array}
$$

and Nef $F=\langle L\rangle$. We have:

- $F$ is a Fano variety.

- $X$ is the complete intersection of two ample divisors on $F$.

- $-\left(K_{F}+\Lambda\right) \sim 2 L$ is ample.

Corollary D.5 yields

and regularizing gives

$$
G_{X}(t)=\sum_{d=0}^{\infty} t^{2 d} \frac{(2 d) !(2 d) !}{(d !)^{6}}
$$

$$
\widehat{G}_{X}(t)=1+8 t^{2}+216 t^{4}+8000 t^{6}+343000 t^{8}+16003008 t^{10}+\cdots .
$$

Minkowski period sequence 75

\section{The Fano manifold $B_{5}$}

Iskovskikh classification This is case 7 in [37, Table 6.5].

Construction A complete intersection $X$ in $\operatorname{Gr}(2,5)$ cut out by a section of $\mathcal{O}(1)^{\oplus 3}$, where $\mathcal{O}(1)$ is the pullback of $\mathcal{O}(1)$ on projective space under the Plücker embedding.

The quantum period The line bundle $\mathcal{O}(1)$ is the ample generator of $\operatorname{Pic}(\operatorname{Gr}(2,5))$, hence $\mathcal{O}(1)$ coincides with $\operatorname{det} S^{\star}$, where $S$ is the universal bundle of subspaces on $\operatorname{Gr}(2,5)$. We apply Theorem F.1 with $a=3$ and $b=c=d=e=0$, obtaining

$$
G_{X}(t)=\sum_{l=0}^{\infty} \sum_{m=0}^{\infty}(-1)^{l+m} t^{2 l+2 m} \frac{((l+m) !)^{3}}{(l !)^{5}(m !)^{5}}\left(1-5(m-l) H_{m}\right),
$$

where $H_{m}$ is the $m^{\text {th }}$ harmonic number. Regularizing yields

$$
\widehat{G}_{X}(t)=1+6 t^{2}+114 t^{4}+2940 t^{6}+87570 t^{8}+2835756 t^{10}+\cdots .
$$

Minkowski period sequence 46

\section{The Fano manifold $V_{2}$}

Iskovskikh classification This is case 8 in [37, Table 6.5]. 
Construction A sextic hypersurface $X$ in $\mathbb{P}(1,1,1,1,3)$.

The quantum period Proposition D.9 yields

$$
G_{X}(t)=e^{-120 t} \sum_{d=0}^{\infty} t^{d} \frac{(6 d) !}{(d !)^{4}(3 d) !}
$$

and regularizing gives

$$
\begin{gathered}
\widehat{G}_{X}(t)=1+68760 t^{2}+55200000 t^{3}+61054781400 t^{4}+71591389125120 t^{5} \\
+88808827978814400 t^{6}+114426010259814758400 t^{7} \\
+151686694219076253783000 t^{8} \\
+205548259807393951744128000 t^{9}+\cdots .
\end{gathered}
$$

Minkowski period sequence None. Note that the anticanonical line bundle of $V_{2}$ is not very ample.

\section{The Fano manifold $V_{4}$}

Iskovskikh classification This is cases 9 and 10 in [37, Table 6.5]. These cases are deformation equivalent: they can both be described as complete intersections of type $(2,4)$ in $\mathbb{P}(1,1,1,1,1,2)$.

Construction A divisor $X$ of degree 4 on $F=\mathbb{P}^{4}$.

The quantum period The toric variety $F$ has weight data

$$
\begin{array}{llllll}
1 & 1 & 1 & 1 & 1 & L
\end{array}
$$

and Nef $F=\langle L\rangle$. We have:

- $F$ is a Fano variety.

- $X \sim 4 L$ is ample.

- $-\left(K_{F}+X\right) \sim L$ is ample.

Corollary D.5 yields

$$
G_{X}(t)=e^{-24 t} \sum_{d=0}^{\infty} t^{d} \frac{(4 d) !}{(d !)^{5}}
$$


and regularizing gives

$$
\begin{aligned}
\widehat{G}_{X}(t)=1+1944 t^{2}+ & 215808 t^{3}+35295192 t^{4}+5977566720 t^{5} \\
+ & 1073491139520 t^{6}+199954313717760 t^{7} \\
& +38302652395770840 t^{8}+7497487505353251840 t^{9}+\cdots .
\end{aligned}
$$

Minkowski period sequence 165

\section{The Fano manifold $V_{6}$}

Iskovskikh classification This is case 11 in [37, Table 6.5].

Construction A codimension- 2 complete intersection $X$ of type $(2 L) \cap(3 L)$ in the toric variety $F=\mathbb{P}^{5}$.

The quantum period The toric variety $F$ has weight data

$$
\begin{array}{llllllll}
1 & 1 & 1 & 1 & 1 & 1 & L
\end{array}
$$

and Nef $F=\langle L\rangle$. We have:

- $F$ is a Fano variety.

- $X$ is the complete intersection of two ample divisors.

- $-\left(K_{F}+\Lambda\right) \sim L$ is ample.

Corollary D.5 yields

and regularizing gives

$$
G_{X}(t)=e^{-12 t} \sum_{d=0}^{\infty} t^{d} \frac{(2 d) !(3 d) !}{(d !)^{6}}
$$

$$
\begin{aligned}
\widehat{G}_{X}(t)=1+396 t^{2} & +17616 t^{3}+1217052 t^{4}+85220640 t^{5} \\
+ & 6349812480 t^{6}+490029523200 t^{7} \\
+ & 38883641777820 t^{8}+3152020367254080 t^{9}+\cdots .
\end{aligned}
$$

Minkowski period sequence 164

\section{The Fano manifold $V_{8}$}

Iskovskikh classification This is case 12 in [37, Table 6.5]. 
Construction A codimension- 3 complete intersection $X$ of type $(2 L) \cap(2 L) \cap(2 L)$ in the toric variety $F=\mathbb{P}^{6}$.

The quantum period The toric variety $F$ has weight data

$$
\begin{array}{lllllllll}
1 & 1 & 1 & 1 & 1 & 1 & 1 & L
\end{array}
$$

and Nef $F=\langle L\rangle$. We have:

- $F$ is a Fano variety.

- $X$ is the complete intersection of three ample divisors.

- $-\left(K_{F}+\Lambda\right) \sim L$ is ample.

Corollary D.5 yields

and regularizing gives

$$
G_{X}(t)=e^{-8 t} \sum_{d=0}^{\infty} t^{d} \frac{(2 d) !(2 d) !(2 d) !}{(d !)^{7}}
$$

$$
\begin{aligned}
\widehat{G}_{X}(t)=1+152 t^{2}+3840 t^{3}+157656 t^{4}+6428160 t^{5}+280064960 t^{6} \\
+12618762240 t^{7}+584579486680 t^{8}+27660007173120 t^{9}+\cdots .
\end{aligned}
$$

Minkowski period sequence 163

\section{The Fano manifold $V_{10}$}

Iskovskikh classification This is case 13 in [37, Table 6.5].

Construction A complete intersection $X$ in $\operatorname{Gr}(2,5)$, cut out by a section of

$$
\mathcal{O}(1)^{\oplus 2} \oplus \mathcal{O}(2)
$$

where $\mathcal{O}(1)$ is the pullback of $\mathcal{O}(1)$ on projective space under the Plücker embedding.

The quantum period We apply Theorem F.1 with $a=2, b=1$ and $c=d=e=0$. This yields

$$
G_{X}(t)=e^{-6 t} \sum_{l=0}^{\infty} \sum_{m=0}^{\infty}(-1)^{l+m} t^{l+m} \frac{((l+m) !)^{2}(2 l+2 m) !}{(l !)^{5}(m !)^{5}}\left(1-5(m-l) H_{m}\right),
$$

where $H_{m}$ is the $m^{\text {th }}$ harmonic number. Regularizing gives

$$
\begin{aligned}
\widehat{G}_{X}(t)=1+78 t^{2}+ & 1320 t^{3}+37746 t^{4}+1051920 t^{5}+31464780 t^{6} \\
+ & 971757360 t^{7}+30859805970 t^{8}+1000739433120 t^{9}+\cdots .
\end{aligned}
$$




\section{Minkowski period sequence 160}

\section{The Fano manifold $V_{12}$}

Iskovskikh classification This is case 14 in [37, Table 6.5].

Construction A subvariety $X$ of $\operatorname{Gr}(2,5)$ cut out by a generic section of $\left(S^{\star} \otimes \operatorname{det} S^{\star}\right) \oplus \operatorname{det} S^{\star}$,

where $S$ is the universal bundle of subspaces on $\operatorname{Gr}(2,5)$.

A remark on the construction Mukai [58] is devoted to this case and it is shown there that $X$ is a complete intersection of 7 hyperplane sections of the (10-dimensional) orthogonal Grassmannian $\operatorname{OGr}(5,10)$ in its spinor embedding in $\mathbb{P}^{15}$. This model contains $X$ as a linear section and, perhaps more important, is the largest hyperplane "un-section" of $X$. Our construction, on the other hand, is better suited for the fast calculation of the quantum period of $X$.

Write $V=\mathbb{C}^{5}$; in what follows, for ease of notation, we denote by $\mathcal{O}(1)$ the line bundle $\operatorname{det} S^{\star}$ on $\operatorname{Gr}(2, V)=\operatorname{Gr}(2,5)$. Let $\Sigma \subset \operatorname{Gr}(2, V)$ be the vanishing locus of a general section $s$ of the vector bundle $S^{\star} \otimes \mathcal{O}(1)$. Below we sketch a general construction of a natural linear embedding $\Sigma \subset \mathrm{OGr}(5,10)$; this shows that our construction and Mukai's construction coincide. To compute the quantum period of $X$, however, we need rather less. Gromov-Witten invariants are deformation-invariant so, since there is a unique deformation family of manifolds of type $V_{12}$ [36; 37], it suffices to show that our construction gives a smooth member of this family. In other words, it suffices to prove that $\Sigma$ is a rank-1 Fano 4 -fold of Fano index 2 - hence coindex 3 in Mukai's terminology — and degree 12 .

The Picard rank of $\Sigma$ is 1 by Sommese's theorem - see Lazarsfeld [46, Theorem 7.1.1] — and, from the exact sequence

$$
\left.\left.0 \rightarrow T_{\Sigma} \rightarrow T_{\mathrm{Gr}(2,5)}\right|_{\Sigma} \rightarrow S^{\star} \otimes \mathcal{O}(1)\right|_{\Sigma} \rightarrow 0
$$

we get that

$$
-K_{\Sigma}=\left.\left(-K_{G r(2,5)} \otimes \bigwedge^{2}(S \otimes \mathcal{O}(-1))\right)\right|_{\Sigma}=\mathcal{O}_{\Sigma}(2) .
$$

That is, $\Sigma$ is a Fano 4 -fold of Fano index 2. It remains to show that $\Sigma$ has degree 12; this is a small calculation in Schubert calculus,

$$
[\Sigma]=c_{2}\left(S^{\star} \otimes \operatorname{det} S^{\star}\right)=\sigma_{1,1}+2 \sigma_{1}^{2}=3 \sigma_{1,1}+2 \sigma_{2}
$$


and, therefore,

$$
\operatorname{deg} \Sigma=[\Sigma] \sigma_{1}^{4}=\sigma_{1,1} \sigma_{1}^{4}+2 \sigma_{1}^{5}=2+10=12 .
$$

We next sketch the promised construction of a linear embedding $\Sigma \subset \operatorname{OGr}(5,10)$. The first task is to construct a rank- 5 vector bundle on $\Sigma$; the bundle that will be the pull-back of the tautological sub-bundle of $\operatorname{OGr}(5,10)$.

We claim that $\operatorname{Ext}_{\Sigma}^{1}\left(S^{\star}, Q\right)=\mathbb{C}$ and take $E$ to be the unique nontrivial extension. To calculate this Ext group consider the Koszul resolution of $\mathcal{O}_{\Sigma}$,

$$
0 \rightarrow \mathcal{O}(-3) \rightarrow S \otimes \mathcal{O}(-1) \rightarrow \mathcal{O}_{\operatorname{Gr}(2, V)} \rightarrow \mathcal{O}_{\Sigma} \rightarrow 0 .
$$

Tensoring by $S \otimes Q$ and using $H^{1}(\operatorname{Gr}(2, V) ; S \otimes Q)=H^{2}(\operatorname{Gr}(2, V) ; S \otimes Q)=\{0\}$ and $H^{2}(\operatorname{Gr}(2, V) ; S \otimes Q \otimes \mathcal{O}(-3))=H^{3}(\operatorname{Gr}(2, V) ; S \otimes Q \otimes \mathcal{O}(-3))=\{0\}$ (both due to the Borel-Weil-Bott theorem) we get

$$
\operatorname{Ext}_{\Sigma}^{1}\left(S^{\star}, Q\right)=H^{1}(\Sigma ; S \otimes Q)=H^{2}(\operatorname{Gr}(2, V) ; S \otimes Q \otimes S \otimes \mathcal{O}(-1))=\mathbb{C},
$$

again by Borel-Weil-Bott.

As anticipated, denote now by $E$ the unique nontrivial rank- 5 extension,

$$
0 \rightarrow Q \rightarrow E \rightarrow S^{\star} \rightarrow 0
$$

The bundle $E$ fits into a natural self-dual "diagram of 9":

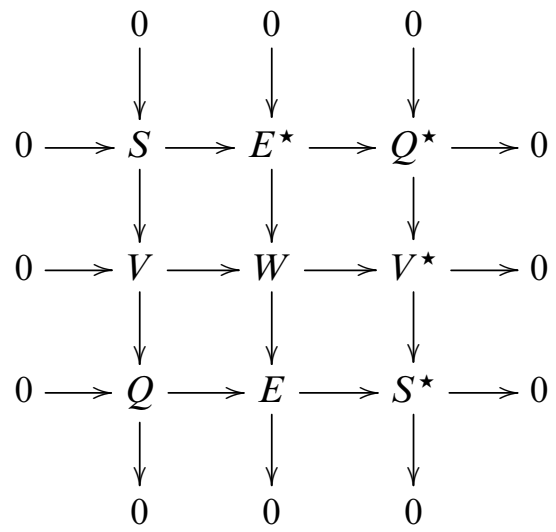

where $W=V \oplus V^{\star}$. The diagram makes it clear that $E \subset V \oplus V^{\star}$ is isotropic when $V \oplus V^{\star}$ is equipped with the canonical nondegenerate symmetric bilinear form. Thus $E$ induces a map $\Sigma \rightarrow \operatorname{OGr}\left(5, V \oplus V^{\star}\right)$. 
The quantum period We apply Theorem F.1 with $a=c=1$ and $b=d=e=0$. This yields

$$
\begin{aligned}
G_{X}(t)=e^{-5 t} \sum_{l, m \geq 0}(-t)^{l+m} \sum_{l=0}^{\infty} \sum_{m=0}^{\infty} \frac{(l+m) !(2 l+m) !(l+2 m) !}{(l !)^{5}(m !)^{5}} \\
\times\left(1+(m-l)\left(H_{2 l+m}+2 H_{l+2 m}-5 H_{m}\right)\right),
\end{aligned}
$$

where $H_{k}$ denotes the $k^{\text {th }}$ harmonic number. Regularizing gives

$$
\begin{aligned}
\widehat{G}_{X}(t)=1+48 t^{2}+600 t^{3}+13176 t^{4}+ & 276480 t^{5}+6259800 t^{6}+146064240 t^{7} \\
& +3505282200 t^{8}+85882130880 t^{9}+\cdots .
\end{aligned}
$$

Minkowski period sequence 150

\section{The Fano manifold $V_{14}$}

Iskovskikh classification This is case 15 in [37, Table 6.5].

Construction A complete intersection $X$ in $\operatorname{Gr}(2,6)$, cut out by a section of $\mathcal{O}(1)^{\oplus 5}$, where $\mathcal{O}(1)$ is the pullback of $\mathcal{O}(1)$ on projective space under the Plücker embedding $[57 ; 30 ; 31]$.

The quantum period We apply Theorem F.1 with $a=5$ and $b=c=d=e=0$. This yields

$$
G_{X}(t)=e^{-4 t} \sum_{l, m \geq 0}(-1)^{l+m} t^{l+m} \frac{((l+m) !)^{5}}{(l !)^{6}(m !)^{6}}\left(1-6(m-l) H_{m}\right),
$$

where $H_{m}$ is the $m^{\text {th }}$ harmonic number. Regularizing gives

$$
\begin{aligned}
\widehat{G}_{X}(t)=1+32 t^{2}+312 t^{3}+5520 t^{4}+91680 t^{5}+1651640 t^{6}+30604560 t^{7} \\
+583436560 t^{8}+11352768000 t^{9}+\cdots .
\end{aligned}
$$

Minkowski period sequence 147

\section{The Fano manifold $V_{16}$}

Iskovskikh classification This is case 16 in [37, Table 6.5]. 
Construction The vanishing locus $X$ of a general section of the vector bundle

$$
\bigwedge^{2} S^{\star} \oplus\left(\operatorname{det} S^{\star}\right)^{\oplus 3}
$$

on $\operatorname{Gr}(3,6)$.

A remark on the construction Mukai [60] is devoted to this case and it is shown there that $X$ is a complete intersection of 3 hyperplane sections of the (6-dimensional) symplectic Grassmannian $\operatorname{SpGr}(3,6)$ of complex Lagrangian 3-dimensional subspaces $W \subset \mathbb{C}^{6}$, where $\mathbb{C}^{6}$ is equipped with the standard symplectic form $\omega \in \wedge^{2} \mathbb{C}^{6 \star}$, in the Plücker embedding inherited from $\operatorname{Gr}(3,6)$. Indeed, the natural surjection $\bigwedge^{2} \mathbb{C}^{6 \star} \rightarrow \wedge^{2} S^{\star}$ induces an isomorphism

$$
H^{0}\left(\operatorname{Gr}(3,6) ; \bigwedge^{2} \mathbb{C}^{6 \star}\right) \cong H^{0}\left(\operatorname{Gr}(3,6) ; \bigwedge^{2} S^{\star}\right)
$$

that allows us to view $\omega$ as an element of $H^{0}\left(\operatorname{Gr}(3,6) ; \bigwedge^{2} S^{\star}\right)$ with zero locus $\operatorname{SpGr}(3,6)$. Thus the construction given above coincides with that given by Mukai [60].

The quantum period We apply Theorem F.1 with $a=3, b=c=d=0$ and $e=1$. This yields

$G_{X}(t)=1+12 t^{2}+32 t^{3}+121 t^{4}+336 t^{5}+\frac{2548}{3} t^{6}+1888 t^{7}+\frac{60481}{16} t^{8}+\frac{185350}{27} t^{9}+\cdots$.

Regularizing gives

$$
\begin{aligned}
\widehat{G}_{X}(t)=1+24 t^{2}+192 t^{3}+2904 t^{4}+40320 t^{5}+611520 t^{6}+9515520 t^{7} \\
+152412120 t^{8}+2491104000 t^{9}+\cdots .
\end{aligned}
$$

Minkowski period sequence 143

\section{The Fano manifold $V_{18}$}

Iskovskikh classification This is case 17 in [37, Table 6.5].

Construction The vanishing locus $X$ of a general section of the vector bundle

$$
\left(S \otimes \operatorname{det} S^{\star}\right) \oplus\left(\operatorname{det} S^{\star}\right)^{\oplus 2}
$$

on $\operatorname{Gr}(5,7)$. 
A remark on the construction The paper [61] is devoted to this case and it is shown there that $X$ is a complete intersection of 2 hyperplane sections of a (5-dimensional) homogeneous space $\Sigma=G_{2} / P$ for the exceptional Lie group $G_{2}$. It is not hard to argue from first principles that $\Sigma$ is the vanishing locus of a general section of $S^{\star} \otimes \operatorname{det} S^{\star}$. We sketch this here, assuming that the reader is acquainted with basic facts about the geometry of the Lie group $G_{2}$. Fix a 7-dimensional complex vector space $V=\mathbb{C}^{7}$ and a 3 -form $\varphi \in \wedge^{3} V^{\star}$ in the generic $\mathrm{GL}_{7}(\mathbb{C})$-orbit; we may take

$$
\varphi=d x^{124}+d x^{235}+d x^{346}+d x^{457}+d x^{561}+d x^{672}+d x^{713},
$$

where $d x^{i j k}=d x^{i} \wedge d x^{j} \wedge d x^{k}$. Then

$$
\Sigma=\left\{W \in \operatorname{Gr}(2, V) \mid \varphi\left(w_{1}, w_{2}, \cdot\right) \equiv 0 \text { for all } w_{1}, w_{2} \in W\right\} .
$$

As usual denote by $0 \rightarrow S \rightarrow V \rightarrow Q \rightarrow 0$ the tautological sequence on $\operatorname{Gr}(2, V)$. Note that $\mathrm{rk} S^{\star}=2$, hence $\wedge^{3} S^{\star}=0$, and therefore there is a natural homomorphism $\bigwedge^{3} V^{\star} \rightarrow Q^{\star} \otimes\left(\bigwedge^{2} S^{\star}\right)$. This homomorphism allows us to

- view $\varphi$ as an element $s_{\varphi} \in H^{0}\left(\operatorname{Gr}(2,7) ; Q^{\star} \otimes \operatorname{det} S^{\star}\right)$; and

- identify $\Sigma$ with $Z\left(s_{\varphi}\right)$.

Finally, we get our construction upon identifying $\operatorname{Gr}(2, V)$ with $\operatorname{Gr}\left(5, V^{\star}\right)$.

The quantum period We apply Theorem F.1 with $a=2, d=1$ and $b=c=e=0$. This yields

$G_{X}(t)=1+9 t^{2}+20 t^{3}+\frac{261}{4} t^{4}+153 t^{5}+\frac{1317}{4} t^{6}+621 t^{7}+\frac{67581}{64} t^{8}+\frac{351641}{216} t^{9}+\cdots$.

Regularizing gives

$$
\begin{aligned}
\widehat{G}_{X}(t)=1+18 t^{2}+120 t^{3}+1566 t^{4}+18360 t^{5} & +237060 t^{6}+3129840 t^{7} \\
& +42576030 t^{8}+590756880 t^{9}+\cdots .
\end{aligned}
$$

Minkowski period sequence 124

\section{The Fano manifold $V_{22}$}

Iskovskikh classification This is case 18 in [37, Table 6.5].

Construction The vanishing locus $X$ of a general section of the vector bundle $\left(S \otimes \operatorname{det} S^{\star}\right)^{\oplus 3}$

on $\operatorname{Gr}(3,7)$ (cf $[56 ; 59])$. 
The quantum period We apply Theorem F.1 with $d=3$ and $a=b=c=e=0$. This yields

$$
G_{X}(t)=1+6 t^{2}+10 t^{3}+\frac{53}{2} t^{4}+48 t^{5}+\frac{977}{12} t^{6}+120 t^{7}+\frac{5117}{32} t^{8}+\frac{5210}{27} t^{9}+\cdots .
$$

Regularizing gives

$$
\begin{aligned}
\widehat{G}_{X}(t)=1+12 t^{2}+60 t^{3}+636 t^{4}+ & 5760 t^{5}+58620 t^{6} \\
& +604800 t^{7}+6447420 t^{8}+70022400 t^{9}+\cdots .
\end{aligned}
$$

Minkowski period sequence 113

\section{The Fano manifold $M_{2-1}$}

Mori-Mukai construction The blow-up of $B_{1}$ with centre an elliptic curve which is the intersection of two members of $\left|-\frac{1}{2} K_{B_{1}}\right|$.

Our construction A divisor $X$ of bidegree $(1,1)$ in the product $\mathbb{P}^{1} \times B_{1}$.

The two constructions coincide Apply Lemma E.1 with $V=\mathcal{O}_{B_{1}} \oplus \mathcal{O}_{B_{1}}, W=$ $-\frac{1}{2} K_{B_{1}}$ and $f: V \rightarrow W$ the map given by the two sections of $-\frac{1}{2} K_{B_{1}}$ that define the elliptic curve.

The quantum period Combining Example G.1, the calculation for $B_{1}$ and Corollary E.4, we have

$$
G_{\mathbb{P}^{1} \times B_{1}}(t)=\sum_{l=0}^{\infty} \sum_{m=0}^{\infty} t^{2 l+2 m} \frac{(6 m) !}{(l !)^{2}(m !)^{3}(2 m) !(3 m) !} .
$$

Applying Remark D.6 yields

$$
G_{X}(t)=e^{-61 t} \sum_{l=0}^{\infty} \sum_{m=0}^{\infty} t^{l+m} \frac{(6 m) !(l+m) !}{(l !)^{2}(m !)^{3}(2 m) !(3 m) !}
$$

and regularizing gives

$$
\begin{aligned}
\widehat{G}_{X}(t)=1+ & 10380 t^{2}+2082840 t^{3}+650599740 t^{4}+199351017360 t^{5} \\
+ & 64604751907800 t^{6}+21521865311226000 t^{7} \\
+ & 7344504146141322300 t^{8}+2554251417295177437600 t^{9}+\cdots .
\end{aligned}
$$

Minkowski period sequence None. Note that the anticanonical line bundle of $X$ is not very ample. 


\section{The Fano manifold $\mathrm{MM}_{2-2}$}

Mori-Mukai construction A double cover of $\mathbb{P}^{1} \times \mathbb{P}^{2}$ branched along a divisor of bidegree $(2,4)$.

Our construction A member $X$ of $|2 L+4 M|$ in the toric variety $F$ with weight data

\begin{tabular}{ccccccc}
$x_{0}$ & $x_{1}$ & $y_{0}$ & $y_{1}$ & $y_{2}$ & $w$ & \\
\cline { 1 - 5 } 1 & 1 & 0 & 0 & 0 & 1 & $L$ \\
0 & 0 & 1 & 1 & 1 & 2 & $M$
\end{tabular}

and Nef $F=\langle L, L+2 M\rangle$. We have:

- $-K_{F}=3 L+5 M$ is ample, that is, $F$ is a smooth Fano orbifold. ${ }^{9}$

- $X \sim 2 L+4 M$ is nef.

- $-\left(K_{F}+X\right) \sim L+M$ is ample.

The two constructions coincide Consider the defining equation of $X$ to be $w^{2}=f_{2,4}$, where $f_{2,4}$ is a bihomogeneous polynomial of degrees 2 in $x_{0}$ and $x_{1}$ and 4 in $y_{0}, y_{1}$ and $y_{2}$. Let $p: F \rightarrow \mathbb{P}^{1} \times \mathbb{P}^{2}$ be the rational map which sends (contravariantly) the homogeneous co-ordinate functions $\left[x_{0}, x_{1}, y_{0}, y_{1}, y_{2}\right]$ on $\mathbb{P}_{x_{0}, x_{1}}^{1} \times \mathbb{P}_{y_{0}, y_{1}, y_{2}}^{2}$ to $\left[x_{0}, x_{1}, y_{0}, y_{1}, y_{2}\right]$. The restriction of $p$ to $X$ is a morphism, which exhibits $X$ as a double cover of $\mathbb{P}^{1} \times \mathbb{P}^{2}$ branched over the locus $\left(f_{2,4}=0\right) \subset \mathbb{P}_{x_{0}, x_{1}}^{1} \times \mathbb{P}_{y_{0}, y_{1}, y_{2}}^{2}$.

Remarks on our construction Next we make some comments on the geometry of $X$ and the embedding $X \subset F$ that are not logically necessary for the computation of the quantum period; this subsection can safely be skipped by the impatient reader. In particular we explain why in this case $\operatorname{Nef} F$ is a proper subset of $\operatorname{Nef} X$. The toric variety $F$ is defined by the requirement that $\operatorname{Nef} F=\langle L, L+2 M\rangle$; the unstable locus is $\left(x_{0}=x_{1}=0\right) \cup\left(y_{0}=y_{1}=y_{2}=w=0\right)$ and

$$
F=\left[\left(\mathbb{C}^{\times}\right)^{2} \times\left(\mathbb{C}^{\times}\right)^{4} / \mathbb{T}^{2}\right] .
$$

Note that $F$ is itself a Fano variety - or, more precisely, a smooth Fano orbifold — and $X$ is a nef divisor on $F$ such that $-\left(K_{F}+X\right)$ is ample, so the given model is well adapted for computing the quantum cohomology of $X$ via quantum Lefschetz. The linear system $|L|=\left|x_{0}, x_{1}\right|$ defines a morphism $f: F \rightarrow \mathbb{P}_{x_{0}, x_{1}}^{1}$ with fibre the weighted

${ }^{9}$ By "smooth orbifold", we mean "smooth Deligne-Mumford stack over $\mathbb{C}$ ". Excellent introductions to Deligne-Mumford stacks can be found in [19; 72, Appendix]; note that in the latter reference DeligneMumford stacks are called "algebraic stacks". By "smooth Fano orbifold", we mean "smooth orbifold such that the coarse moduli space is a Fano variety". 
projective space $\mathbb{P}(1,1,1,2)$; the restriction $\left.f\right|_{X}: X \rightarrow \mathbb{P}^{1}$ is one of the two extremal contractions of $X$. On the other hand, the linear system $|M|=\left|y_{0}, y_{1}, y_{2}\right|$ is not basepoint-free on $F$ : the base locus is a section $C$ of the morphism $f$. When restricted to $X$, however, this linear system is free and it defines the "other" extremal contraction $X \rightarrow \mathbb{P}^{2}$. In particular, we see that $\langle L, L+2 M\rangle=\operatorname{Nef} F \subsetneq \operatorname{Nef} X=\langle L, M\rangle$. How can we see the rest of Nef $X$ ?

Let us denote by $F^{\prime}$ the toric variety corresponding to the "other" chamber, so that Nef $F^{\prime}=\langle L+2 M, M\rangle$ and the unstable locus is $\left(y_{0}=y_{1}=y_{2}=0\right) \cup\left(x_{0}=x_{1}=w=0\right)$. Note that $F^{\prime}$ is the flip of $F$ along the curve $C=\left(y_{0}=y_{1}=y_{2}=0\right) \subset F . X$ is a member of $|2 L+4 M|$, a nef linear system on $F^{\prime}$, but $-\left(K_{F^{\prime}}+X\right)$ is not nef on $F^{\prime}$ and so this construction of $X$ is not well adapted for computing the quantum cohomology of $X$ via quantum Lefschetz. Nevertheless, Nef $X=\operatorname{Nef} F+\operatorname{Nef} F^{\prime}$, so we need $F^{\prime}$ to see all of $\operatorname{Nef} X$. The linear system $\left|y_{0}, y_{1}, y_{2}\right|$ is free on $F^{\prime}$ and it defines an extremal contraction $g: F^{\prime} \rightarrow \mathbb{P}^{2}$ with fibre $\mathbb{P}^{2}$; this also gives the missing extremal contraction of $X$.

The quantum period If we assume a mirror theorem for toric orbifolds in the form [35, Conjecture 4.3] then we can apply the quantum Lefschetz theorem for orbifolds [13], exactly as in Proposition D.9, to obtain

Regularizing gives

$$
G_{X}(t)=e^{-14 t} \sum_{l=0}^{\infty} \sum_{m=0}^{\infty} t^{l+m} \frac{(2 l+4 m) !}{(l !)^{2}(m !)^{3}(l+2 m) !} .
$$

$$
\begin{aligned}
\widehat{G}_{X}(t)=1+470 t^{2} & +21216 t^{3}+1562778 t^{4} \\
+ & 114717120 t^{5}+9003183140 t^{6}+731280419520 t^{7} \\
+ & 61092935052730 t^{8}+5214279501137280 t^{9}+\cdots .
\end{aligned}
$$

Minkowski period sequence None. Note that the anticanonical line bundle of $X$ is not very ample.

The quantum period, alternative construction There is as yet no proof of $[35$, Conjecture 4.3] in the literature, so we give an alternative calculation of the quantum period for $X$. This uses a different model of $X$, as a member of $|2 N|$ in the toric variety $F$ with weight data

\begin{tabular}{cccccccc}
$x_{0}$ & $x_{1}$ & $y_{0}$ & $y_{1}$ & $y_{2}$ & $w$ & $z$ & \\
\cline { 1 - 6 } 1 & 1 & 0 & 0 & 0 & -1 & 0 & $L$ \\
0 & 0 & 1 & 1 & 1 & -2 & 0 & $M$ \\
0 & 0 & 0 & 0 & 0 & 1 & 1 & $N$
\end{tabular}


and Nef $F=\langle L, M, N\rangle$. The variety $F$ is the projective bundle

$$
\mathbb{P}\left(\mathcal{O}_{\mathbb{P}^{1} \times \mathbb{P}^{2}}(-1,-2) \oplus \mathcal{O}_{\mathbb{P}^{1} \times \mathbb{P}^{2}}\right)
$$

over $\mathbb{P}^{2}$. Let $p: F \rightarrow \mathbb{P}^{1} \times \mathbb{P}^{2}$ be the projection map and consider the defining equation of $X$ to be

$$
z^{2}-w^{2} f_{2,4}=0
$$

where $f_{2,4}$ is a bihomogeneous polynomial of degrees 2 in $x_{0}$ and $x_{1}$ and 4 in $y_{0}, y_{1}$ and $y_{2}$. The restriction $\left.p\right|_{X}: X \rightarrow \mathbb{P}^{1} \times \mathbb{P}^{2}$ exhibits $X$ as a double cover of $\mathbb{P}^{1} \times \mathbb{P}^{2}$ branched over the locus $\left(f_{2,4}=0\right) \subset \mathbb{P}_{x_{0}, x_{1}}^{1} \times \mathbb{P}_{y_{0}, y_{1}, y_{2}}^{2}$.

We now compute the quantum period of $X$. Let $p_{1}, p_{2}, p_{3} \in H^{\bullet}(F ; \mathbb{Z})$ denote the first Chern classes of $L, M$ and $N$, respectively; these classes form a basis for $H^{2}(F ; \mathbb{Z})$. Write $\tau \in H^{2}(F ; \mathbb{Q})$ as $\tau=\tau_{1} p_{1}+\tau_{2} p_{2}+\tau_{3} p_{3}$ and identify the group ring $\mathbb{Q}\left[H_{2}(F ; \mathbb{Z})\right]$ with the polynomial ring $\mathbb{Q}\left[Q_{1}, Q_{2}, Q_{3}\right]$ via the $\mathbb{Q}$-linear map that sends the element $Q^{\beta} \in \mathbb{Q}\left[H_{2}(F ; \mathbb{Z})\right]$ to $Q_{1}^{\left\langle\beta, p_{1}\right\rangle} Q_{2}^{\left\langle\beta, p_{2}\right\rangle} Q_{3}^{\left\langle\beta, p_{3}\right\rangle}$. The toric variety $F$ is Fano; Theorem C.1 gives

$$
\begin{array}{r}
J_{F}(\tau)=e^{\tau / z} \sum_{l, m, n \geq 0} \frac{Q_{1}^{l} Q_{2}^{m} Q_{3}^{n} e^{l \tau_{1}} e^{m \tau_{2}} e^{n \tau_{3}}}{\prod_{k=1}^{l}\left(p_{1}+k z\right)^{2} \prod_{k=1}^{m}\left(p_{2}+k z\right)^{3} \prod_{k=1}^{n}\left(p_{3}+k z\right)} \\
\times \frac{\prod_{k=-\infty}^{0}\left(p_{3}-p_{1}-2 p_{2}+k z\right)}{\prod_{k=-\infty}^{n-l-2 m}\left(p_{3}-p_{1}-2 p_{2}+k z\right)}
\end{array}
$$

and hence

$$
\begin{array}{r}
I_{e, E}(\tau)=e^{\tau / z} \sum_{l, m, n \geq 0} \frac{Q_{1}^{l} Q_{2}^{m} Q_{3}^{n} e^{l \tau_{1}} e^{m \tau_{2}} e^{n \tau_{3}} \prod_{k=1}^{2 n}\left(\lambda+2 p_{3}+k z\right)}{\prod_{k=1}^{l}\left(p_{1}+k z\right)^{2} \prod_{k=1}^{m}\left(p_{2}+k z\right)^{3} \prod_{k=1}^{n}\left(p_{3}+k z\right)} \\
\times \frac{\prod_{k=-\infty}^{0}\left(p_{3}-p_{1}-2 p_{2}+k z\right)}{\prod_{k=-\infty}^{n-l-2 m}\left(p_{3}-p_{1}-2 p_{2}+k z\right)}
\end{array}
$$

We have

$$
I_{\boldsymbol{e}, E}(\tau)=A(\tau)+B(\tau) z^{-1}+O\left(z^{-2}\right),
$$

where

$$
A(\tau)=\sum_{n=0}^{\infty} Q_{3}^{n} e^{n \tau_{3}} \frac{(2 n) !}{(n !)^{2}}=\left(1-4 Q_{3} e^{\tau_{3}}\right)^{-\frac{1}{2}}
$$

$$
\begin{aligned}
B(\tau)=\sum_{n=1}^{\infty} Q_{1} e^{\tau_{1}} & Q_{3}^{n} e^{n \tau_{3}} \frac{(2 n) !}{n !(n-1) !}+\sum_{n=2}^{\infty} Q_{2} e^{\tau_{2}} Q_{3}^{n} e^{n \tau_{3}} \frac{(2 n) !}{n !(n-2) !} \\
& +\sum_{n=0}^{\infty} Q_{3}^{n} e^{n \tau_{3}} \frac{(2 n) !}{(n !)^{2}}\left(\left(\lambda+2 p_{3}\right) H_{2 n}-p_{3} H_{n}-\left(p_{3}-p_{1}-2 p_{2}\right) H_{n}\right)
\end{aligned}
$$


and $H_{m}$ is the $m^{\text {th }}$ harmonic number. In the notation of Corollary D.4, we have

$$
\begin{aligned}
A(\tau)= & \left(1-4 Q_{3} e^{\tau_{3}}\right)^{-\frac{1}{2}} \\
B^{\prime}(\tau)= & \sum_{n=1}^{\infty} Q_{1} e^{\tau_{1}} Q_{3}^{n} e^{n \tau_{3}} \frac{(2 n) !}{n !(n-1) !}+\sum_{n=2}^{\infty} Q_{2} e^{\tau_{2}} Q_{3}^{n} e^{n \tau_{3}} \frac{(2 n) !}{n !(n-2) !} \\
& \quad+\sum_{n=0}^{\infty} Q_{3}^{n} e^{n \tau_{3}} \frac{(2 n) !}{(n !)^{2}}\left(p_{3}\left(2 H_{2 n}-H_{n}\right)-\left(p_{3}-p_{1}-2 p_{2}\right) H_{n}\right) \\
=2 Q_{1} e^{\tau_{1}} Q_{3} e^{\tau_{3}}\left(1-4 Q_{3} e^{\tau_{3}}\right)^{-\frac{3}{2}}+12 Q_{2} e^{\tau_{2}} Q_{3}^{2} e^{2 \tau_{3}}\left(1-4 Q_{3} e^{\tau_{3}}\right)^{-\frac{5}{2}} & \quad-p_{3}\left(1-4 Q_{3} e^{\tau_{3}}\right)^{-\frac{1}{2}} \log \left(1-4 Q_{3} e^{\tau_{3}}\right) \\
& \quad-\left(p_{3}-p_{1}-2 p_{2}\right) \sum_{n=0}^{\infty} Q_{3}^{n} e^{n \tau_{3}} \frac{(2 n) !}{(n !)^{2}} H_{n} .
\end{aligned}
$$

Corollary D.4 gives

$$
J_{Y, X}(\theta(\tau))=\left(1-4 Q_{3} e^{\tau_{3}}\right)^{\frac{1}{2}} I_{Y, X}(\tau),
$$

where

$$
\begin{aligned}
& \theta(\tau)=\tau+ \frac{2 Q_{1} e^{\tau_{1}} Q_{3} e^{\tau_{3}}}{1-4 Q_{3} e^{\tau_{3}}}+\frac{12 Q_{2} e^{\tau_{2}} Q_{3}^{2} e^{2 \tau_{3}}}{\left(1-4 Q_{3} e^{\tau_{3}}\right)^{2}} \\
& \quad-p_{3} \log \left(1-4 Q_{3} e^{\tau_{3}}\right)-\left(p_{3}-p_{1}-2 p_{2}\right) F, \\
& F=\left(1-4 Q_{3} e^{\tau_{3}}\right)^{\frac{1}{2}} \sum_{n=0}^{\infty} Q_{3}^{n} e^{n \tau_{3}} \frac{(2 n) !}{(n !)^{2}} H_{n}
\end{aligned}
$$

and

$$
\begin{array}{r}
I_{Y, X}(\tau)=e^{\tau / z} \sum_{l, m, n \geq 0} \frac{Q_{1}^{l} Q_{2}^{m} Q_{3}^{n} e^{l \tau_{1}} e^{m \tau_{2}} e^{n \tau_{3}} \prod_{k=1}^{2 n}\left(2 p_{3}+k z\right)}{\prod_{k=1}^{l}\left(p_{1}+k z\right)^{2} \prod_{k=1}^{m}\left(p_{2}+k z\right)^{3} \prod_{k=1}^{n}\left(p_{3}+k z\right)} \\
\times \frac{\prod_{k=-\infty}^{0}\left(p_{3}-p_{1}-2 p_{2}+k z\right)}{\prod_{k=-\infty}^{n-l-2 m}\left(p_{3}-p_{1}-2 p_{2}+k z\right)} .
\end{array}
$$

From (8), we have that

$$
j_{\star} J_{X}\left(j^{\star} \theta(\tau)\right)=2 p_{3}\left(1-4 Q_{3} e^{\tau_{3}}\right)^{1 / 2} I_{Y, X}(\tau),
$$

where $j: X \rightarrow F$ is the inclusion map and equality holds after applying the map of coefficient rings $\Lambda_{X} \rightarrow \Lambda_{F}$ induced by $j$. Note that $j^{\star}\left(p_{3}-p_{1}-2 p_{2}\right)=0$; this reflects the fact that $X$ is disjoint from the divisor $w=0$. Consider the classes $p_{1}^{\prime}=j^{\star} p_{1}$ and $p_{2}^{\prime}=j^{\star} p_{2}$. These form a basis for $H^{2}(X)$, and we identify the group ring $\mathbb{Q}\left[H_{2}(X ; \mathbb{Z})\right]$ with the polynomial ring $\mathbb{Q}\left[q_{1}, q_{2}\right]$ via the $\mathbb{Q}$-linear map that sends the element $Q^{\beta} \in \mathbb{Q}\left[H_{2}(F ; \mathbb{Z})\right]$ to $q_{1}^{\left\langle\beta, p_{1}^{\prime}\right\rangle} q_{2}^{\left\langle\beta, p_{2}^{\prime}\right\rangle}$. The map $\Lambda_{X} \rightarrow \Lambda_{F}$ 
induced by $j$ sends $q_{1}$ to $Q_{1} Q_{3}$ and $q_{2}$ to $Q_{2} Q_{3}^{2}$. We have

$$
\begin{aligned}
j^{\star} \theta(\tau)=\left(\tau_{1}+\tau_{3}\right) p_{1}^{\prime}+\left(\tau_{2}+2 \tau_{3}\right) p_{2}^{\prime}+\frac{2 Q_{1} e^{\tau_{1}} Q_{3} e^{\tau_{3}}}{1-4 Q_{3} e^{\tau_{3}}}+\frac{12 Q_{2} e^{\tau_{2}} Q_{3}^{2} e^{2 \tau_{3}}}{\left(1-4 Q_{3} e^{\tau_{3}}\right)^{2}} \\
-\left(p_{1}^{\prime}+2 p_{2}^{\prime}\right) \log \left(1-4 Q_{3} e^{\tau_{3}}\right)
\end{aligned}
$$

and thus, from (3)

$$
\begin{aligned}
& J_{X}\left(j^{\star} \theta(\tau)\right) \\
& =\exp \left(\left(\frac{2 Q_{1} e^{\tau_{1}} Q_{3} e^{\tau_{3}}}{1-4 Q_{3} e^{\tau_{3}}}+\frac{12 Q_{2} e^{\tau_{2}} Q_{3}^{2} e^{2 \tau_{3}}}{\left(1-4 Q_{3} e^{\tau_{3}}\right)^{2}}\right) z^{-1}\right) \\
& \quad \times\left. J_{X}\left(\left(\tau_{1}+\tau_{3}\right) p_{1}^{\prime}+\left(\tau_{2}+2 \tau_{3}\right) p_{2}^{\prime}\right)\right|_{Q_{1}=\frac{Q_{1}}{1-4 Q_{3} e^{\tau_{3}}}, Q_{2}=\frac{Q_{2}}{\left(1-4 Q_{3} e^{\left.\tau_{3}\right)^{2}}\right.}}
\end{aligned}
$$

Making the inverse change of variables $Q_{1}=Q_{1}\left(1-4 Q_{3} e^{\tau_{3}}\right), Q_{2}=Q_{2}\left(1-4 Q_{3} e^{\tau_{3}}\right)^{2}$, we see that ${ }^{10}$

$$
\begin{aligned}
j_{\star} J_{X}(0)=e^{-\left(2 Q_{1} Q_{3}+12 Q_{2} Q_{3}^{2}\right) / z} & \times\left. 2 p_{3}\left(1-4 Q_{3}\right)^{\frac{1}{2}} I_{Y, X}(0)\right|_{Q_{1}=Q_{1}\left(1-4 Q_{3}\right), Q_{2}=Q_{2}\left(1-4 Q_{3}\right)^{2}} .
\end{aligned}
$$

Recall that the quantum period $G_{X}$ is obtained from the component of $J_{X}(0)$ along the unit class $1 \in H^{\bullet}(X ; \mathbb{Q})$ by setting $z=1$ and $Q^{\beta}=t^{\left\langle\beta,-K_{X}\right\rangle}$. To obtain $G_{X}$, therefore, we need to extract the coefficient of $2 p_{3}$ on the right-hand side of (15) and set $z=1$,

$$
Q_{1} Q_{2}=t \quad \text { and } \quad Q_{1} Q_{3}^{2}=t
$$

(this amounts to setting $q_{1}=q_{2}=t$ and then applying the map of coefficient rings $\Lambda_{X} \rightarrow \Lambda_{F}$ induced by the inclusion $\left.j\right)$. Observe that $p_{3}\left(p_{3}-p_{1}-2 p_{2}\right)=0$ in $H^{\bullet}(F)$. Taking the coefficient of $2 p_{3}$ on the right-hand side of (15) and setting $z=1$ thus gives

$$
\begin{gathered}
e^{-\left(2 Q_{1} Q_{3}+12 Q_{2} Q_{3}^{2}\right)} \sum_{l=0}^{\infty} \sum_{m=0}^{\infty} \sum_{n=l+2 m}^{\infty} Q_{1}^{l} Q_{2}^{m} Q_{3}^{n}\left(1-4 Q_{3}\right)^{l+2 m+\frac{1}{2}} \\
\times \frac{(2 n) !}{(l !)^{2}(m !)^{3} n !(n-l-2 m) !} \\
=e^{-\left(2 Q_{1} Q_{3}+12 Q_{2} Q_{3}^{2}\right)} \sum_{l=0}^{\infty} \sum_{m=0}^{\infty} \frac{Q_{1}^{l} Q_{2}^{m} Q_{3}^{l+2 m}\left(1-4 Q_{3}\right)^{l+2 m+\frac{1}{2}}}{(l !)^{2}(m !)^{3}} \\
\times \sum_{n=l+2 m}^{\infty} Q_{3}^{n-l-2 m} \frac{(2 n) !}{n !(n-l-2 m) !}
\end{gathered}
$$

${ }^{10}$ The right-hand side of (15) depends on $Q_{1}, Q_{2}$ and $Q_{3}$ only through the products $Q_{1} Q_{3}$ and $Q_{2} Q_{3}^{2}$, but this is not manifest from the formula. We will see it explicitly below for the coefficient of $2 p_{3}$ in (15). 


$$
\begin{aligned}
& =e^{-\left(2 Q_{1} Q_{3}+12 Q_{2} Q_{3}^{2}\right)} \sum_{l=0}^{\infty} \sum_{m=0}^{\infty} \frac{Q_{1}^{l} Q_{2}^{m} Q_{3}^{l+2 m}\left(1-4 Q_{3}\right)^{l+2 m+\frac{1}{2}}}{(l !)^{2}(m !)^{3}} \times\left(\frac{d}{d Q_{3}}\right)^{l+2 m}\left(1-4 Q_{3}\right)^{-\frac{1}{2}} \\
& =e^{-\left(2 Q_{1} Q_{3}+12 Q_{2} Q_{3}^{2}\right)} \sum_{l=0}^{\infty} \sum_{m=0}^{\infty} Q_{1}^{l} Q_{2}^{m} Q_{3}^{l+2 m} \frac{(2 l+4 m) !}{(l !)^{2}(m !)^{3}(l+2 m) !} .
\end{aligned}
$$

Setting $Q_{1} Q_{3}=t, Q_{1} Q_{3}^{2}=t$ yields

$$
G_{X}(t)=e^{-14 t} \sum_{l=0}^{\infty} \sum_{m=0}^{\infty} t^{l+m} \frac{(2 l+4 m) !}{(l !)^{2}(m !)^{3}(l+2 m) !}
$$

and regularizing gives (14), as before.

\section{The Fano manifold $\mathrm{MM}_{2-3}$}

Mori-Mukai construction The blow-up of $B_{2}$ with centre an elliptic curve that is the intersection of two members of $\left|-\frac{1}{2} K_{B_{2}}\right|$.

Our construction A divisor $X$ of bidegree $(1,1)$ in the product $\mathbb{P}^{1} \times B_{2}$.

The two constructions coincide Apply Lemma E.1 with $V=\mathcal{O}_{B_{2}} \oplus \mathcal{O}_{B_{2}}, W=$ $-\frac{1}{2} K_{B_{2}}$ and $f: V \rightarrow W$ the map given by the two sections of $-\frac{1}{2} K_{B_{2}}$ that define the elliptic curve.

The quantum period Combining Example G.1, the calculation for $B_{2}$ and Corollary E.4, we have

$$
G_{\mathbb{P}^{1} \times B_{2}}(t)=\sum_{l=0}^{\infty} \sum_{m=0}^{\infty} t^{2 l+2 m} \frac{(4 m) !}{(l !)^{2}(m !)^{4}(2 m) !} .
$$

Applying Remark D.6 yields

$$
G_{X}(t)=e^{-13 t} \sum_{l=0}^{\infty} \sum_{m=0}^{\infty} t^{l+m} \frac{(4 m) !(l+m) !}{(l !)^{2}(m !)^{4}(2 m) !}
$$

and regularizing gives

$$
\begin{aligned}
\widehat{G}_{X}(t)=1+ & 300 t^{2}+8472 t^{3}+438588 t^{4}+21183120 t^{5}+1115221080 t^{6} \\
& +60512230800 t^{7}+3385779824700 t^{8}+193681282922400 t^{9}+\cdots .
\end{aligned}
$$


Minkowski period sequence None. Note that the anticanonical line bundle of $X$ is not very ample.

\section{The Fano manifold $\mathrm{MM}_{2-4}$}

Mori-Mukai construction The blow-up of $\mathbb{P}^{3}$ with centre an intersection of two cubics.

Our construction A member $X$ of $|L+3 M|$ in the toric variety $F=\mathbb{P}^{1} \times \mathbb{P}^{3}$.

The two constructions coincide Apply Lemma E.1 with $V=\mathcal{O}_{\mathbb{P}^{3}} \oplus \mathcal{O}_{\mathbb{P}} 3, W=$ $\mathcal{O}_{\mathbb{P}^{3}}(3)$ and $f: V \rightarrow W$ given by the two cubics that define the centre of the blow-up.

The quantum period The toric variety $F$ has weight data

$$
\begin{aligned}
& \begin{array}{llllllll}
1 & 1 & 0 & 0 & 0 & 0 & L
\end{array} \\
& \begin{array}{llllllll}
0 & 0 & 1 & 1 & 1 & 1 & M
\end{array}
\end{aligned}
$$

and Nef $F=\langle L, M\rangle$. We have:

- $F$ is a Fano variety.

- $X \sim L+3 M$ is ample.

- $-\left(K_{F}+X\right) \sim L+M$ is ample.

Corollary D.5 yields

$$
G_{X}(t)=e^{-7 t} \sum_{l=0}^{\infty} \sum_{m=0}^{\infty} t^{l+m} \frac{(l+3 m) !}{(l !)^{2}(m !)^{4}}
$$

and regularizing gives

$$
\begin{aligned}
\widehat{G}_{X}(t)=1+90 t^{2}+ & 1518 t^{3}+46086 t^{4}+1327320 t^{5}+41383350 t^{6} \\
+ & 1329442380 t^{7}+43944315030 t^{8}+1483208104560 t^{9}+\cdots .
\end{aligned}
$$

Minkowski period sequence 161

\section{The Fano manifold $\mathrm{MM}_{2-5}$}

Mori-Mukai construction The blow-up of $B_{3}$ with centre a plane cubic on it. 
Our construction A member $X$ of $|3 M|$ in the toric variety $F$ with weight data

\begin{tabular}{ccccccc}
$s_{0}$ & $s_{1}$ & $x$ & $x_{2}$ & $x_{3}$ & $x_{4}$ & \\
\cline { 1 - 5 } 1 & 1 & -1 & 0 & 0 & 0 & \\
0 & 0 & 1 & 1 & 1 & 1 & $M$
\end{tabular}

and Nef $F=\langle L, M\rangle$. We have:

- $-K_{F}=L+4 M$ is ample, that is, $F$ is a Fano variety.

- $\quad X \sim 3 M$ is nef.

- $-\left(K_{F}+X\right) \sim L+M$ is ample.

The two constructions coincide The notation makes it clear that $s_{0}$ and $s_{1}$ are sections of $L ; x s_{0}, x s_{1}, x_{2}, x_{3}$ and $x_{4}$ are sections of $M$; and $F$ is a scroll over $\mathbb{P}^{1}$ with fibre $\mathbb{P}^{3}$. The morphism $F \rightarrow \mathbb{P}^{4}$ that sends (contravariantly) the homogeneous co-ordinate functions $\left[x_{0}, \ldots, x_{4}\right]$ to $\left[x s_{0}, x s_{1}, x_{2}, x_{3}, x_{4}\right]$ is the blowup along $x_{0}=x_{1}=0$.

The quantum period Corollary D.5 yields

and regularizing gives

$$
G_{X}(t)=e^{-6 t} \sum_{l=0}^{\infty} \sum_{m=l}^{\infty} t^{l+m} \frac{(3 m) !}{(l !)^{2}(m-l) !(m !)^{3}}
$$

$$
\begin{aligned}
& \widehat{G}_{X}(t)=1+66 t^{2}+816 t^{3}+20214 t^{4}+449640 t^{5}+11050500 t^{6} \\
&+ 278336520 t^{7}+7229175030 t^{8}+191680807920 t^{9}+\cdots .
\end{aligned}
$$

Minkowski period sequence 158

\section{The Fano manifold $\mathrm{MM}_{2-6}$}

Mori-Mukai construction Either

(a) a divisor of bidegree $(2,2)$ on $\mathbb{P}^{2} \times \mathbb{P}^{2}$; or

(b) a double cover of $W \subset \mathbb{P}^{2} \times \mathbb{P}^{2}$ (the divisor of bidegree $(1,1)$ on $\mathbb{P}^{2} \times \mathbb{P}^{2}$ ) whose branch locus is a member of $\left|-K_{W}\right|$.

Our construction A member $X$ of $|2 L+2 M|$ in the toric variety $F=\mathbb{P}^{2} \times \mathbb{P}^{2}$.

The two constructions coincide Obvious. 
The quantum period This is Example D.7. We have

$$
\begin{aligned}
\widehat{G}_{X}(t)=1+44 t^{2}+528 t^{3} & +11292 t^{4}+228000 t^{5}+4999040 t^{6} \\
& +112654080 t^{7}+2613620380 t^{8}+61885803840 t^{9}+\cdots .
\end{aligned}
$$

Minkowski period sequence 149

\section{The Fano manifold $\mathrm{MM}_{2-7}$}

Mori-Mukai construction The blow-up of a quadric 3 -fold $Q \subset \mathbb{P}^{4}$ with centre the intersection of two members of $\left|\mathcal{O}_{Q}(2)\right|$.

Our construction A codimension-2 complete intersection $X$ of type

$$
(2 M) \cap(L+2 M)
$$

on the toric variety $F=\mathbb{P}^{1} \times \mathbb{P}^{4}$.

The two constructions coincide Apply Lemma E.1 with $V=\mathcal{O}_{Q} \oplus \mathcal{O}_{Q}, W=\mathcal{O}_{Q}(2)$ and $f: V \rightarrow W$ given by the two sections of $\mathcal{O}_{Q}(2)$ that define the centre of the blowup. This shows that $X$ is a divisor of bidegree $(1,2)$ on $\mathbb{P}^{1} \times Q$ or, in other words, a complete intersection of type $(2 M) \cap(L+2 M)$ on $\mathbb{P}^{1} \times \mathbb{P}^{4}$.

The quantum period The toric variety $F$ has weight data

$$
\begin{aligned}
& \begin{array}{lllllllll}
1 & 1 & 0 & 0 & 0 & 0 & 0 & L
\end{array} \\
& \begin{array}{lllllllll}
0 & 0 & 1 & 1 & 1 & 1 & 1 & M
\end{array}
\end{aligned}
$$

and Nef $F=\langle L, M\rangle$. We have:

- $F$ is a Fano variety.

- $X$ is the complete intersection of two nef divisors on $F$.

- $-\left(K_{F}+\Lambda\right) \sim L+M$ is ample.

Corollary D.5 yields

and regularizing gives

$$
G_{X}(t)=e^{-5 t} \sum_{l=0}^{\infty} \sum_{m=0}^{\infty} t^{l+m} \frac{(2 m) !(l+2 m) !}{(l !)^{2}(m !)^{5}}
$$

$$
\begin{aligned}
\widehat{G}_{X}(t)=1+36 t^{2}+348 t^{3} & +6516 t^{4}+110880 t^{5}+2069820 t^{6} \\
& +39606000 t^{7}+780530100 t^{8}+15697106880 t^{9}+\cdots .
\end{aligned}
$$




\section{Minkowski period sequence 148}

\section{The Fano manifold $\mathrm{MM}_{2-8}$}

\section{Mori-Mukai construction}

(i) A double cover of $B_{7}$ (the blow-up of $\mathbb{P}^{3}$ at a point) with branch locus a member $B$ of $\left|-K_{B_{7}}\right|$ such that $B \cap D$ is nonsingular, where $D$ is the exceptional divisor of the blow-up $B_{7} \rightarrow \mathbb{P}^{3}$.

(ii) A specialization of (a) where $B \cap D$ is reduced but singular.

Our construction A member $X$ of $|2 L+2 M|$ in the toric variety $F$ with weight data

\begin{tabular}{ccccccc}
$s_{0}$ & $s_{1}$ & $s_{2}$ & $x$ & $x_{3}$ & $w$ & \\
\cline { 1 - 5 } 1 & 1 & 1 & -1 & 0 & 1 & $L$ \\
0 & 0 & 0 & 1 & 1 & 1 & $M$
\end{tabular}

and Nef $F=\langle L, L+M\rangle$. We have:

- $-K_{F}=3(L+M)$ is nef and big but not ample, so that $F$ is not a Fano variety.

- $\quad X \sim 2(L+M)$ is nef.

- $-\left(K_{F}+X\right) \sim L+M$ is nef and big but not ample.

The two constructions coincide Consider the equation of $X$ in the form

$$
w^{2}=x_{3}^{2} a_{2}+x_{3} x b_{3}+x^{2} c_{4},
$$

where $a_{2}, b_{3}$, and $c_{4}$ are generic homogeneous polynomials in $s_{0}, s_{1}$ and $s_{2}$ of degrees 2,3 and 4 , respectively. The locus $(w=0) \subset F$ is a copy of $B_{7}$ and the branch locus meets the exceptional divisor $D=(x=w=0) \cong \mathbb{P}^{2}$ in a nonsingular conic.

Remarks on the birational geometry of $\boldsymbol{X}$ Next we make a few comments on the geometry of $X$ and the embedding $X \subset F$ that are not logically necessary for the computation of the quantum period. The discussion is similar to the discussion of $\mathrm{MM}_{2-2}$ above; it in particular shows that $X$ is a Fano variety, which is not immediately clear from our construction.

The secondary fan manifestly has three maximal cones. By definition, Nef $F=$ $\langle L, L+M\rangle$. The irrelevant ideal is $\left(w s_{i}, x_{3} s_{i}, x s_{i}\right)$ and the unstable locus is

$$
\left(s_{0}=s_{1}=s_{2}=0\right) \cup\left(w=x=x_{3}=0\right) .
$$


The linear system $|L|=\left|s_{0}, s_{1}, s_{2}\right|$ defines a morphism $f: F \rightarrow \mathbb{P}^{2}$ with fibre $\mathbb{P}^{2}$ and $\left.f\right|_{X}$ is a conic bundle (in particular, an extremal contraction in the Mori category). The linear system $|L+M|=\left|w, x_{3} s_{i}, x s_{i} s_{j}\right|$ gives a flopping contraction of $\Pi=$ $\left(x=x_{3}=0\right) \cong \mathbb{P}^{2}$ with normal bundle $\mathcal{O}(-1) \oplus \mathcal{O}(-2)$. Note, however, that $X \cap \Pi=\varnothing$ : this contraction maps $X$ isomorphically onto its image.

Denote by $F^{\prime}$ the toric variety such that Nef $F^{\prime}=\langle L+M, M\rangle$. The irrelevant ideal is

$$
\left(x_{3} w, x w, x_{3} s_{i}, x s_{i}\right)
$$

and the unstable locus is

$$
\left(x=x_{3}=0\right) \cup\left(s_{0}=s_{1}=s_{2}=w=0\right) .
$$

The linear system $|L+M|$ defines the flop of $F$. On the other hand, $|M|$ defines a contraction $g: F \rightarrow \mathbb{P}(1,1,1,1,2)$ which sends (contravariantly) the homogeneous co-ordinate functions $\left[x_{0}, x_{1}, x_{2}, x_{3}, y\right]$ on $\mathbb{P}(1,1,1,1,2)$ to $\left[s_{0} x, s_{1} x, s_{2} x, x_{3}, w x\right]$. The restriction $\left.g\right|_{X}$ maps $X$ to the variety $Y$ with equation

$$
y^{2}=x_{3}^{2} a_{2}\left(x_{0}, x_{1}, x_{2}\right)+x_{3} b_{3}\left(x_{0}, x_{1}, x_{2}\right)+c_{4}\left(x_{0}, x_{1}, x_{2}\right),
$$

so $Y$ is the double cover of $\mathbb{P}^{3}$ branched along a general quartic surface $B$ with an ordinary node at $(0,0,0,1)$ and $\left.g\right|_{X}: X \rightarrow Y$ is an extremal divisorial contraction contracting $X \cap(x=0)=\left(w^{2}=x_{3}^{2} a_{2}\left(s_{0}, s_{1}, s_{2}\right)\right) \cong \mathbb{P}^{1} \times \mathbb{P}^{1}$ to the node just mentioned.

It follows from the preceding discussion that $\operatorname{Nef} X=\operatorname{Nef} F+\operatorname{Nef} F^{\prime}=\langle L, M\rangle$; in particular, therefore, $X$ is Fano.

Finally the chamber $\langle M, M-L\rangle$ is "hollow", that is, taking the GIT quotient with respect to a stability condition from the interior of this chamber leads to a rank-1 toric variety.

The quantum period Let $p_{1}, p_{2} \in H^{\bullet}(F ; \mathbb{Z})$ denote the first Chern classes of $L$ and $L \otimes M$, respectively; these classes form a basis for $H^{2}(F ; \mathbb{Z})$. Write $\tau \in H^{2}(F ; \mathbb{Q})$ as $\tau=\tau_{1} p_{1}+\tau_{2} p_{2}$ and identify the group ring $\mathbb{Q}\left[H_{2}(F ; \mathbb{Z})\right]$ with the polynomial ring $\mathbb{Q}\left[Q_{1}, Q_{2}\right]$ via the $\mathbb{Q}$-linear map that sends the element $Q^{\beta} \in \mathbb{Q}\left[H_{2}(F ; \mathbb{Z})\right]$ to $Q_{1}^{\left\langle\beta, p_{1}\right\rangle} Q_{2}^{\left\langle\beta, p_{2}\right\rangle}$. We have

$$
\begin{gathered}
I_{F}(\tau)=e^{\tau / z} \sum_{l, m \geq 0} \frac{Q_{1}^{l} Q_{2}^{m} e^{l \tau_{1}} e^{m \tau_{2}}}{\prod_{k=1}^{l}\left(p_{1}+k z\right)^{3} \prod_{k=1}^{m}\left(p_{2}+k z\right)} \\
\times \frac{\prod_{k=-\infty}^{0}\left(p_{2}-p_{1}+k z\right)}{\prod_{k=-\infty}^{m-l}\left(p_{2}-p_{1}+k z\right)} \frac{\prod_{k=-\infty}^{0}\left(p_{2}-2 p_{1}+k z\right)}{\prod_{k=-\infty}^{m-2 l}\left(p_{2}-2 p_{1}+k z\right)} \\
=1+\tau z^{-1}+O\left(z^{-2}\right) .
\end{gathered}
$$


Assumptions D.1 hold and, in the notation of Proposition D.2, we have $A(\tau)=1$ and $B(\tau)=\tau$. We now proceed exactly as in the proof of Corollary D.5, obtaining

$$
\begin{array}{r}
I_{F, X}(\tau)=e^{\tau / z} \sum_{l, m \geq 0} \frac{Q_{1}^{l} Q_{2}^{m} e^{l \tau_{1}} e^{m \tau_{2}} \prod_{k=1}^{2 l+2 m}\left(2 p_{1}+2 p_{2}+k z\right)}{\prod_{k=1}^{l}\left(p_{1}+k z\right)^{3} \prod_{k=1}^{m}\left(p_{2}+k z\right)} \\
\times \frac{\prod_{k=-\infty}^{0}\left(p_{2}-p_{1}+k z\right)}{\prod_{k=-\infty}^{m-l}\left(p_{2}-p_{1}+k z\right)} \frac{\prod_{k=-\infty}^{0}\left(p_{2}-2 p_{1}+k z\right)}{\prod_{k=-\infty}^{m-2 l}\left(p_{2}-2 p_{1}+k z\right)}
\end{array}
$$

and

$$
G_{X}(t)=e^{-2 t} \sum_{l=0}^{\infty} \sum_{m=2 l}^{\infty} t^{m} \frac{(2 m) !}{(l !)^{3} m !(m-l) !(m-2 l) !} .
$$

Regularizing gives

$$
\begin{aligned}
\widehat{G}_{X}(t)=1+26 t^{2}+216 t^{3}+ & 3582 t^{4}+54480 t^{5}+874700 t^{6} \\
& +15000720 t^{7}+256965310 t^{8}+4576672800 t^{9}+\cdots .
\end{aligned}
$$

Minkowski period sequence 144

\section{The Fano manifold $M_{2-9}$}

Mori-Mukai construction The blow-up of $\mathbb{P}^{3}$ with centre a curve $\Gamma$ of degree 7 and genus 5 that is an intersection of cubics.

Our construction A codimension-2 complete intersection $X$ of type

$$
(L+M) \cap(2 L+M)
$$

in the toric variety $F=\mathbb{P}^{3} \times \mathbb{P}^{2}$.

The two constructions coincide The curve $\Gamma$ is cut out by the equations:

$$
\operatorname{rk}\left(\begin{array}{ccc}
l_{0} & l_{1} & l_{2} \\
q_{0} & q_{1} & q_{2}
\end{array}\right)<2
$$

where the $l_{i}$ are linear forms and the $q_{j}$ are quadratic forms. Lemma E.1 implies that $X$ is the complete intersection given by the two equations

$$
\left\{\begin{aligned}
l_{0} y_{0}+l_{1} y_{1}+l_{2} y_{2} & =0, \\
q_{0} y_{0}+q_{1} y_{1}+q_{2} y_{2} & =0,
\end{aligned}\right.
$$

in $\mathbb{P}^{3} \times \mathbb{P}^{2}$, where the first factor has co-ordinates $x_{0}, x_{1}, x_{2}, x_{3}$ and the second factor has co-ordinates $y_{0}, y_{1}, y_{2}$. In other words, $X$ is a complete intersection of type $(L+M) \cap(2 L+M)$ in $\mathbb{P}^{3} \times \mathbb{P}^{2}$. 
The quantum period The toric variety $F$ has weight data

$$
\begin{array}{cccccccc}
1 & 1 & 1 & 1 & 0 & 0 & 0 & \\
0 & 0 & 0 & 0 & 1 & 1 & 1 & M
\end{array}
$$

and Nef $F=\langle L, M\rangle$. We have:

- $F$ is a Fano variety.

- $X$ is the complete intersection of two ample divisors on $F$.

- $-\left(K_{F}+\Lambda\right) \sim L+M$ is ample.

Corollary D.5 yields

$$
G_{X}(t)=e^{-3 t} \sum_{l=0}^{\infty} \sum_{m=0}^{\infty} t^{l+m} \frac{(l+m) !(2 l+m) !}{(l !)^{4}(m !)^{3}}
$$

and regularizing gives

$$
\begin{aligned}
\widehat{G}_{X}(t)=1+22 t^{2}+174 t^{3}+ & 2514 t^{4}+34200 t^{5}+501070 t^{6} \\
+ & 7586880 t^{7}+117858370 t^{8}+1870811040 t^{9}+\cdots .
\end{aligned}
$$

Minkowski period sequence 139

\section{The Fano manifold $M_{2-10}$}

Mori-Mukai construction The blow-up of $B_{4} \subset \mathbb{P}^{5}$ with centre an elliptic curve that is an intersection of two hyperplane sections.

Our construction A codimension- 2 complete intersection $X$ of type $(2 M) \cap(2 M)$ in the toric variety $F$ with weight data

\begin{tabular}{cccccccc}
$s_{0}$ & $s_{1}$ & $x$ & $x_{2}$ & $x_{3}$ & $x_{4}$ & $x_{5}$ & \\
\cline { 1 - 5 } 1 & 1 & -1 & 0 & 0 & 0 & 0 & $L$ \\
0 & 0 & 1 & 1 & 1 & 1 & 1 & $M$
\end{tabular}

and Nef $F=\langle L, M\rangle$. We have:

- $-K_{F}=L+5 M$ is ample, that is, $F$ is a Fano variety.

- $X$ is the complete intersection of two nef divisors on $F$.

- $-\left(K_{F}+\Lambda\right) \sim L+M$ is ample. 
The two constructions coincide The notation makes it clear that $s_{0}$ and $s_{1}$ are sections of $L ; x s_{0}, x s_{1}, x_{2}, x_{3}, x_{4}$ and $x_{5}$ are sections of $M$; and $F$ is a scroll over $\mathbb{P}^{1}$ with fibre $\mathbb{P}^{4}$. The morphism $F \rightarrow \mathbb{P}^{4}$ that sends (contravariantly) the homogeneous coordinate functions $\left[x_{0}, x_{1}, x_{2}, x_{3}, x_{4}, x_{5}\right]$ to $\left[x s_{0}, x s_{1}, x_{2}, x_{3}, x_{4}, x_{5}\right]$ is the blow-up along $\left(x_{0}=x_{1}=0\right) \subset \mathbb{P}^{4}$.

The quantum period Corollary D.5 yields

and regularizing gives

$$
G_{X}(t)=e^{-4 t} \sum_{l=0}^{\infty} \sum_{m=l}^{\infty} t^{l+m} \frac{(2 m) !(2 m) !}{(l !)^{2}(m-l) !(m !)^{4}}
$$

$$
\begin{aligned}
\widehat{G}_{X}(t)=1+28 t^{2}+216 t^{3}+ & 3516 t^{4}+49680 t^{5}+783640 t^{6} \\
& +12594960 t^{7}+208898620 t^{8}+3533634720 t^{9}+\cdots .
\end{aligned}
$$

Minkowski period sequence 145

\section{The Fano manifold $\mathrm{MM}_{2-11}$}

Mori-Mukai construction The blow-up of $B_{3} \subset \mathbb{P}^{4}$ with centre a line on it.

Our construction A member $X$ of $|L+2 M|$ in the toric variety $F$ with weight data

\begin{tabular}{ccccccc}
$s_{0}$ & $s_{1}$ & $s_{2}$ & $x$ & $x_{3}$ & $x_{4}$ & \\
\cline { 1 - 5 } & 1 & 1 & -1 & 0 & 0 & $L$ \\
0 & 0 & 0 & 1 & 1 & 1 & $M$
\end{tabular}

and Nef $F=\langle L, M\rangle$. We have:

- $-K_{F}=2 L+3 M$ is ample, that is, $F$ is a Fano variety.

- $X \sim L+2 M$ is ample.

- $-\left(K_{F}+X\right) \sim L+M$ is ample.

The two constructions coincide The notation makes it clear that $s_{0}, s_{1}$ and $s_{2}$ are sections of $L ; x s_{0}, x s_{1}, x s_{2}, x_{3}$ and $x_{4}$ are sections of $M$; and $F$ is a scroll over $\mathbb{P}^{2}$ with fibre $\mathbb{P}^{2}$. The morphism $F \rightarrow \mathbb{P}^{4}$ that sends (contravariantly) the homogeneous coordinate functions $\left[x_{0}, x_{1}, x_{2}, x_{3}, x_{4}\right]$ to $\left[x s_{0}, x s_{1}, x s_{2}, x_{3}, x_{4}\right]$ is the blow-up along the line $\ell=\left(x_{0}=x_{1}=x_{2}=0\right) \subset \mathbb{P}^{4}$. We construct $X$ as the proper transform of a general cubic $B_{3} \subset \mathbb{P}^{4}$ containing the line $\ell$. This $B_{3}$ has an equation of the form

$$
x_{0} A+x_{1} B+x_{2} C=0,
$$


where $A, B$ and $C$ are homogeneous quadratic polynomials in the variables $x_{0}, \ldots, x_{4}$. Thus $X$ is given in $F$ by the equation:

$s_{0} A\left(s_{0} x, s_{1} x, s_{2} x, x_{3}, x_{4}\right)+s_{1} B\left(s_{0} x, s_{1} x, s_{2} x, x_{3}, x_{4}\right)+s_{2} C\left(s_{0} x, s_{1} x, s_{2} x, x_{3}, x_{4}\right)=0$

The quantum period Corollary D.5 yields

and regularizing gives

$$
G_{X}(t)=e^{-2 t} \sum_{l=0}^{\infty} \sum_{m=l}^{\infty} t^{l+m} \frac{(l+2 m) !}{(l !)^{3}(m-l) !(m !)^{2}}
$$

$$
\begin{aligned}
\widehat{G}_{X}(t)=1+14 t^{2}+108 t^{3}+1074 t^{4}+13440 t^{5}+154760 t^{6} \\
+1951320 t^{7}+24999730 t^{8}+325321920 t^{9}+\cdots .
\end{aligned}
$$

\section{Minkowski period sequence 120}

\section{The Fano manifold $\mathrm{MM}_{2-12}$}

Mori-Mukai construction The blow-up of $\mathbb{P}^{3}$ with centre a curve $\Gamma$ of degree 6 and genus 3 that is an intersection of cubics.

Our construction A codimension- 3 complete intersection $X$ of type

$$
(L+M) \cap(L+M) \cap(L+M)
$$

in the toric variety $F=\mathbb{P}^{3} \times \mathbb{P}^{3}$.

The two constructions coincide The curve $\Gamma \subset \mathbb{P}_{x_{0}, x_{1}, x_{3}}^{3}$ is given by the condition

$$
\mathrm{rk}\left(\begin{array}{llll}
l_{00} & l_{01} & l_{02} & l_{03} \\
l_{10} & l_{11} & l_{12} & l_{13} \\
l_{20} & l_{21} & l_{22} & l_{23}
\end{array}\right)<3
$$

where the $l_{i j}$ are linear forms in $x_{0}, \ldots, x_{3}$. Lemma E. 1 implies that $X$ is a codimension- 3 complete intersection in $\mathbb{P}_{x_{0}, x_{1}, x_{2}, x_{3}}^{3} \times \mathbb{P}_{y_{0}, y_{1}, y_{2}, y_{3}}^{3}$ given by the three equations

$$
\left\{\begin{array}{l}
l_{00} y_{0}+l_{01} y_{1}+l_{02} y_{2}+l_{03} y_{3}=0 \\
l_{10} y_{0}+l_{11} y_{1}+l_{12} y_{2}+l_{13} y_{3}=0 \\
l_{20} y_{0}+l_{21} y_{1}+l_{22} y_{2}+l_{23} y_{3}=0 .
\end{array}\right.
$$

In other words, $X$ is a complete intersection of type $(L+M) \cap(L+M) \cap(L+M)$ in $\mathbb{P}^{3} \times \mathbb{P}^{3}$. An equivalent description of this variety was given by Qureshi [67, Proposition 6.4.1]. 
The quantum period The toric variety $F$ has weight data

$$
\begin{array}{ccccccccc}
1 & 1 & 1 & 1 & 0 & 0 & 0 & 0 & L \\
0 & 0 & 0 & 0 & 1 & 1 & 1 & 1 & M
\end{array}
$$

and Nef $F=\langle L, M\rangle$. We have that:

- $F$ is a Fano variety.

- $X$ is the complete intersection of three ample divisors on $F$.

- $-\left(K_{F}+\Lambda\right) \sim L+M$ is ample.

Corollary D.5 yields

and regularizing gives

$$
G_{X}(t)=e^{-2 t} \sum_{l=0}^{\infty} \sum_{m=0}^{\infty} t^{l+m} \frac{((l+m) !)^{3}}{(l !)^{4}(m !)^{4}}
$$

$$
\begin{aligned}
\widehat{G}_{X}(t)=1+14 t^{2}+72 t^{3}+882 t^{4} & +8400 t^{5}+95180 t^{6} \\
& +1060080 t^{7}+12389650 t^{8}+146472480 t^{9}+\cdots .
\end{aligned}
$$

Minkowski period sequence 118

\section{The Fano manifold $\mathrm{MM}_{2-13}$}

Mori-Mukai construction The blow-up of a quadric 3 -fold $Q \subset \mathbb{P}^{4}$ with centre a curve $\Gamma$ of degree 6 and genus 2 .

Our construction A codimension- 3 complete intersection $X$ of type

$$
(L+M) \cap(L+M) \cap(2 M)
$$

in the toric variety $F=\mathbb{P}^{2} \times \mathbb{P}^{4}$.

The two constructions coincide Let $\left[s_{0}, s_{1}, y\right]$ be homogeneous co-ordinates on $\mathbb{P}(1,1,3)$. We have that $\Gamma=\mathbb{P}(1,1,3) \cap Q$, where the embedding $\mathbb{P}(1,1,3) \hookrightarrow \mathbb{P}^{4}$ sends (contravariantly) the homogeneous co-ordinate functions

$$
\left[x_{0}, \ldots, x_{4}\right] \mapsto\left[s_{0}^{3}, s_{0}^{2} s_{1}, s_{0} s_{1}^{2}, s_{1}^{3}, y\right] .
$$

Thus $\mathbb{P}(1,1,3) \subset \mathbb{P}^{4}$ is given by the condition

$$
\operatorname{rk}\left(\begin{array}{lll}
x_{0} & x_{1} & x_{2} \\
x_{1} & x_{2} & x_{3}
\end{array}\right)<2
$$


By Lemma E.1, the blow-up $G$ of $\mathbb{P}^{4}$ along $\mathbb{P}(1,1,3)$ is the complete intersection in $\mathbb{P}_{y_{0}, \ldots, y_{2}}^{2} \times \mathbb{P}_{x_{0}, \ldots, x_{4}}^{4}$ cut out by the equations

$$
\left\{\begin{array}{l}
x_{0} y_{0}-x_{1} y_{1}+x_{2} y_{2}=0 \\
x_{1} y_{0}-x_{2} y_{1}+x_{3} y_{2}=0
\end{array}\right.
$$

Our Fano variety $X$ is the complete intersection of $G$ with a quadric $q\left(x_{0}, x_{1}, x_{2}, x_{3}, x_{4}\right)$. Thus $X$ is a complete intersection of type $(L+M) \cap(L+M) \cap(2 M)$ in $\mathbb{P}^{2} \times \mathbb{P}^{4}$.

The quantum period The toric variety $F$ has weight data

$$
\begin{array}{lllllllll}
1 & 1 & 1 & 0 & 0 & 0 & 0 & 0 & \\
0 & 0 & 0 & 1 & 1 & 1 & 1 & 1 & M
\end{array}
$$

and Nef $F=\langle L, M\rangle$. We have that:

- $F$ is a Fano variety.

- $X$ is the complete intersection of three nef divisors on $F$.

- $-\left(K_{F}+\Lambda\right) \sim L+M$ is ample.

Corollary D.5 yields

and regularizing gives

$$
G_{X}(t)=e^{-3 t} \sum_{l=0}^{\infty} \sum_{m=0}^{\infty} t^{l+m} \frac{(2 m) !((l+m) !)^{2}}{(l !)^{3}(m !)^{5}}
$$

$$
\begin{aligned}
\widehat{G}_{X}(t)=1+14 t^{2}+84 t^{3}+930 t^{4} & +9720 t^{5}+108680 t^{6} \\
& +1259160 t^{7}+14951650 t^{8}+181377840 t^{9}+\cdots .
\end{aligned}
$$

Minkowski period sequence 119

\section{The Fano manifold $\mathrm{MM}_{2-14}$}

Mori-Mukai construction The blow-up of $B_{5} \subset \mathbb{P}^{6}$ with centre an elliptic curve that is an intersection of two hyperplane sections.

Our construction A divisor ${ }^{11} X$ of bidegree $(1,1)$ on $B_{5} \times \mathbb{P}^{1}$.

${ }^{11}$ This is one of six cases of families of rank-2 Fano 3-folds $\left(\mathrm{MM}_{2-14}, \mathrm{MM}_{2-17}, \mathrm{MM}_{2-20}\right.$, $\left.\mathrm{MM}_{2-21}, \mathrm{MM}_{2-22}, \mathrm{MM}_{2-26}\right)$ where the generic member is not a complete intersection in a toric variety. Of these, four $\left(\mathrm{MM}_{2-14}, \mathrm{MM}_{2-20}, \mathrm{MM}_{2-22}, \mathrm{MM}_{2-26}\right)$ are blow-ups of $B_{5}$ along a curve: a complete intersection, a twisted cubic, a conic and a line. Fano 3-folds in families $\mathrm{MM}_{2-17}$ and $\mathrm{MM}_{2-21}$ are blow-ups of a quadric 3-fold. 
The two constructions coincide Let $\left[x_{0}, \ldots, x_{6}\right]$ be homogeneous co-ordinates on $\mathbb{P}^{6}$ and let $F \rightarrow \mathbb{P}^{6}$ be the blow-up in the complete intersection $\left(x_{0}=x_{1}=0\right)$. Our Fano variety $X$ is the proper transform of $B_{5} \subset \mathbb{P}^{6}$ under the blow-up. Applying Lemma E.1 with $V=\mathcal{O}_{\mathbb{P}^{6}} \oplus \mathcal{O}_{\mathbb{P}^{6}}, W=\mathcal{O}_{\mathbb{P}^{6}}(1)$ and $f: V \rightarrow W$ the map given by $\left(x_{0}, x_{1}\right)$ shows that $F$ is the subvariety of $\mathbb{P}_{x_{0}, \ldots, x_{6}}^{6} \times \mathbb{P}_{y_{0}, y_{1}}^{1}$ given by the equation $x_{0} y_{0}+x_{1} y_{1}=0$.

The quantum period Combining Example G.1, the calculation for $B_{5}$ and Corollary E.4, we have

$$
G_{B_{5} \times \mathbb{P}^{1}}(t)=\sum_{l=0}^{\infty} \sum_{m=0}^{\infty} \sum_{n=0}^{\infty}(-1)^{l+m} t^{2 l+2 m+2 n} \frac{((l+m) !)^{3}}{(l !)^{5}(m !)^{5}(n !)^{2}}\left(1-5(m-l) H_{m}\right),
$$

where $H_{m}$ is the $m^{\text {th }}$ harmonic number. Applying Remark D.6 yields

$G_{X}(t)=e^{-4 t} \sum_{l=0}^{\infty} \sum_{m=0}^{\infty} \sum_{n=0}^{\infty}(-1)^{l+m} t^{l+m+n} \frac{(l+m+n) !((l+m) !)^{3}}{(l !)^{5}(m !)^{5}(n !)^{2}}\left(1-5(m-l) H_{m}\right)$

and regularizing gives

$$
\begin{aligned}
\widehat{G}_{X}(t)=1+16 t^{2}+90 t^{3}+1104 t^{4}+11460 t^{5}+133990 t^{6} \\
+1588860 t^{7}+19463920 t^{8}+242996040 t^{9}+\cdots .
\end{aligned}
$$

Minkowski period sequence 122

\section{The Fano manifold $\mathbf{M M}_{2-15}$}

Mori-Mukai construction The blow-up of $\mathbb{P}^{3}$ with centre the intersection of a quadric $A$ and a cubic $B$.

Our construction A member $X$ of $|2 L+M|$ in the toric variety $F$ with weight data

\begin{tabular}{rrrrrrr}
$s_{0}$ & $s_{1}$ & $s_{2}$ & $s_{3}$ & $x$ & $x_{4}$ & \\
\cline { 1 - 5 } & 1 & 1 & 1 & -1 & 0 & $L$ \\
0 & 0 & 0 & 0 & 1 & 1 & $M$
\end{tabular}

and Nef $F=\langle L, M\rangle$. We have:

- $-K_{F}=3 L+2 M$ is ample, that is, $F$ is a Fano variety.

- $X \sim 2 L+M$ is ample.

- $-\left(K_{F}+X\right) \sim L+M$ is ample. 
The two constructions coincide Apply Lemma E.1 with $V=\mathcal{O}_{\mathbb{P}^{3}}(-1) \oplus \mathcal{O}_{\mathbb{P}^{3}}$, $W=\mathcal{O}_{\mathbb{P}^{3}}(2)$ and $f: V \rightarrow W$ the map given by the matrix $(B A)$.

The quantum period Corollary D.5 yields

and regularizing gives

$$
G_{X}(t)=e^{-t} \sum_{l=0}^{\infty} \sum_{m=l}^{\infty} t^{l+m} \frac{(2 l+m) !}{(l !)^{4}(m-l) ! m !}
$$

$$
\begin{aligned}
\widehat{G}_{X}(t)=1+12 t^{2}+36 t^{3}+564 t^{4}+ & 3600 t^{5}+41700 t^{6} \\
& +360360 t^{7}+3839220 t^{8}+37749600 t^{9}+\cdots .
\end{aligned}
$$

Minkowski period sequence 109

\section{The Fano manifold $\mathrm{MM}_{2-16}$}

Mori-Mukai construction The blow-up of $B_{4} \subset \mathbb{P}^{5}$ with centre a conic on it.

Our construction A codimension- 2 complete intersection $X$ of type $(L+M) \cap(2 M)$ in the toric variety $F$ with weight data

\begin{tabular}{cccccccc}
$s_{0}$ & $s_{1}$ & $s_{2}$ & $x$ & $x_{3}$ & $x_{4}$ & $x_{5}$ & \\
\cline { 1 - 5 } & 1 & 1 & -1 & 0 & 0 & 0 & $L$ \\
0 & 0 & 0 & 1 & 1 & 1 & 1 & $M$
\end{tabular}

and Nef $F=\langle L, M\rangle$. We have:

- $-K_{F}=2 L+4 M$ is ample, that is $F$ is a Fano variety.

- $X$ is the complete intersection of two nef divisors on $F$.

- $-\left(K_{F}+\Lambda\right) \sim L+M$ is ample.

The two constructions coincide The morphism $F \rightarrow \mathbb{P}^{5}$ that sends (contravariantly) the homogeneous co-ordinate functions $\left[x_{0}, x_{1}, \ldots, x_{5}\right]$ to $\left[s_{0} x, s_{1} x, s_{2} x, x_{3}, x_{4}, x_{5}\right]$ blows up the plane $\Pi=\left(x_{0}=x_{1}=x_{2}=0\right)$. We realise $X$ as the complete intersection of the proper transform of a quadric containing $\Pi$ and a generic quadric.

The quantum period Corollary D.5 yields

$$
G_{X}(t)=e^{-2 t} \sum_{l=0}^{\infty} \sum_{m=l}^{\infty} t^{l+m} \frac{(l+m) !(2 m) !}{(l !)^{3}(m-l) !(m !)^{3}}
$$


and regularizing gives

$$
\begin{aligned}
\widehat{G}_{X}(t)=1+10 t^{2}+60 t^{3}+510 t^{4}+ & 4920 t^{5}+47080 t^{6} \\
& +473760 t^{7}+4908190 t^{8}+51641520 t^{9}+\cdots .
\end{aligned}
$$

Minkowski period sequence 104

\section{The Fano manifold $\mathbf{M M}_{2-17}$}

Mori-Mukai construction The blow-up of a quadric 3-fold $Q \subset \mathbb{P}^{4}$ with centre an elliptic curve $\Gamma$ of degree 5 on it.

Our construction The vanishing locus $X$ of a general section of the vector bundle

$$
\left(S^{\star} \otimes \mathcal{O}_{\mathbb{P}^{3}}(1)\right) \oplus\left(\operatorname{det} S^{\star} \otimes \mathcal{O}_{\mathbb{P}^{3}}(1)\right) \oplus\left(\operatorname{det} S^{\star} \otimes \mathcal{O}_{\mathbb{P}^{3}}\right)
$$

on the key variety $F=\operatorname{Gr}(2,4) \times \mathbb{P}^{3}$, where $S$ is the universal bundle of subspaces on $\operatorname{Gr}(2,4)$.

The two constructions coincide First consider $\operatorname{Gr}(2,4)$ with tautological rank- 2 subbundle $S \subset \mathbb{C}^{4}$ : it is well known that the vanishing locus $Z=Z(s)$ of a general section $s \in \Gamma(\operatorname{Gr}(2,4) ; E)$, where

$$
E=S^{\star} \otimes \operatorname{det} S^{\star}
$$

is a del Pezzo surface of degree 5. Indeed, this can be shown as follows: the adjunction formula immediately implies that $-K_{Z}=-\left.\left(K_{X} \otimes \operatorname{det} E\right)\right|_{Z}=\operatorname{det} S^{\star}$ is ample, that is, $Z$ is a del Pezzo surface, and a small exercise in Schubert calculus shows that $K_{Z}^{2}=5$.

Next we blow up $Z \subset \operatorname{Gr}(2,4)$. Consider the $\mathbb{P}^{1}$-bundle $p: \mathbb{P}\left(E^{\star}\right) \rightarrow \operatorname{Gr}(2,4)$ of lines in $E^{\star}$ : under $p^{\star} E \rightarrow \mathcal{O}(1)$ we can identify $s \in \Gamma(\operatorname{Gr}(2,4) ; E)=\Gamma\left(\mathbb{P}\left(E^{\star}\right), \mathcal{O}(1)\right)$ with a section $\tilde{s}$ of $\mathcal{O}(1)$ on $\mathbb{P}\left(E^{\star}\right)$ and, by Lemma E.1,

$$
p: Y=Z(\tilde{s}) \subset \mathbb{P}\left(E^{\star}\right) \rightarrow \operatorname{Gr}(2,4) \quad \text { blows up } Z=Z(s) \subset \operatorname{Gr}(2,4) .
$$

Next, identify:

- $\mathbb{P}\left(E^{\star}\right)=\mathbb{P}(S \otimes \operatorname{det} S)$ with $\mathbb{P}(S)$. Write $V=\mathbb{C}^{4}$ with basis $e_{0}, \ldots, e_{3}$ and note that the tautological sequence

$$
0 \rightarrow S \rightarrow V \rightarrow Q \rightarrow 0
$$

on $\operatorname{Gr}(2,4)$ identifies $V^{\star}$ with $\Gamma\left(\operatorname{Gr}(2,4) ; S^{\star}\right)$. In this notation, we can now also identify

$$
\mathbb{P}(S)=Z(\sigma) \subset \operatorname{Gr}(2, V) \times \mathbb{P}\left(V^{\star}\right)
$$


where $\sigma=e_{0} x_{0}+\cdots e_{3} x_{3} \in \Gamma\left(\operatorname{Gr}(2, V) \times \mathbb{P}\left(V^{\star}\right) ; S^{\star} \otimes \mathcal{O}(1)\right)$ is a general section.

- The line bundle $\mathcal{O}(1)$ on $\mathbb{P}\left(E^{\star}\right)$ with the line bundle $\operatorname{det} S^{\star}(1)$ on $\mathbb{P}(S)$ and $\tilde{s}$ with a section that, abusing notation, we still denote by $\tilde{s}$ :

$$
\tilde{s} \in \Gamma\left(\mathbb{P}(S) ; \operatorname{det} S^{\star}(1)\right) .
$$

Combining all of the above we identify the blow-up $Y$ of a del Pezzo surface of degree $5, Z \subset \operatorname{Gr}(2,4)$, with the vanishing locus of a general section $(\sigma, \tilde{s})$ of the bundle

$$
\left(S^{\star} \otimes \mathcal{O}_{\mathbb{P}^{3}}(1)\right) \oplus\left(\operatorname{det} S^{\star} \otimes \mathcal{O}_{\mathbb{P}^{3}}(1)\right)
$$

on $\operatorname{Gr}(2,4) \times \mathbb{P}^{3}$. It follows easily from this that our construction and the Mori-Mukai construction coincide.

Abelianization Consider $\operatorname{Gr}(2,4)$ as the geometric quotient $\mathbb{C}^{8} / / \mathrm{GL}_{2}(\mathbb{C})$, where we regard $\mathbb{C}^{8}$ as the space $M(2,4)$ of $2 \times 4$ complex matrices and $\mathrm{GL}_{2}(\mathbb{C})$ acts by multiplication on the left. The universal bundle $S$ of subspaces on $\operatorname{Gr}(2,4)$ is the bundle on $\mathbb{C}^{8} / / \mathrm{GL}_{2}(\mathbb{C})$ determined by $V_{\text {std }}^{\star}$, where $V_{\text {std }}$ is the standard representation of $\mathrm{GL}_{2}(\mathbb{C})$. Consider the situation as in $[9$, Section 3.1] with

- the space that is denoted by $X$ in [9] set equal to $A=\mathbb{C}^{12}$, regarded as the space of pairs

$$
\left\{(M, w) \mid M \text { is a } 2 \times 4 \text { complex matrix and } w \in \mathbb{C}^{4} \text { is a vector }\right\} ;
$$

- $G=\mathrm{GL}_{2}(\mathbb{C}) \times \mathbb{C}^{\times}$, acting on $A$ as

$$
(g, \lambda):(M, w) \mapsto(g M, \lambda w) ;
$$

- $T=\left(\mathbb{C}^{\times}\right)^{3}$, the diagonal subtorus in $G$;

- the group that is denoted by $S$ in [9] set equal to the trivial group;

- $\mathcal{V}$ equal to the representation of $G$ given by

$$
\left(V_{\text {std }} \otimes V_{\text {std }}\right) \oplus\left(\operatorname{det} V_{\text {std }} \otimes V_{\text {std }}\right) \oplus \operatorname{det} V_{\text {std }} \otimes V_{\text {triv }},
$$

where $V_{\text {triv }}$ is the trivial 1-dimensional representation of $\mathbb{C}^{\times}$.

It is clear that $A / / G=F$, whereas $A / / T=\mathbb{P}^{3} \times \mathbb{P}^{3} \times \mathbb{P}^{3}$. The non-trivial element in the Weyl group $W=\mathbb{Z} / 2 \mathbb{Z}$ permutes the first and second factors in the product $\mathbb{P}^{3} \times \mathbb{P}^{3} \times \mathbb{P}^{3}$. The representation $\mathcal{V}$ induces the vector bundle $\mathcal{V}_{G}=E$ over $F$, whereas the representation $\mathcal{V}$ induces the vector bundle

$$
\mathcal{V}_{T}=\mathcal{O}(1,0,1) \oplus \mathcal{O}(0,1,1) \oplus \mathcal{O}(1,1,1) \oplus \mathcal{O}(1,1,0)
$$

over $A / / T$. 
The abelian/non-abelian correspondence Let $p_{i} \in H^{2}(A / / T ; \mathbb{Q}), 1 \leq i \leq 3$, denote the first Chern class of $\pi_{i}^{\star} \mathcal{O}_{\mathbb{P}^{3}}(1)$, where $\pi_{i}: A / / T \rightarrow \mathbb{P}^{3}$ is projection to the $i^{\text {th }}$ factor of the product $A / / T=\mathbb{P}^{3} \times \mathbb{P}^{3} \times \mathbb{P}^{3}$. Set $\Omega=\left(p_{2}-p_{1}\right)$. We fix a lift of $H^{\bullet}(A / / G ; \mathbb{Q})$ to $H^{\bullet}(A / / T, \mathbb{Q})^{W}$ in the sense of [9, Section 3]. As in the proof of Theorem F.1 there are many possible choices for such a lift and the precise choice made will be unimportant in what follows. The lift allows us to regard $H^{\bullet}(A / / G ; \mathbb{Q})$ as a subspace of $H^{\bullet}(A / / T, \mathbb{Q})^{W}$, which maps isomorphically to the Weyl-anti-invariant part $H^{\bullet}(A / / T, \mathbb{Q})^{a}$ of $H^{\bullet}(A / / T, \mathbb{Q})$ via

$$
H^{\bullet}(A / / T, \mathbb{Q})^{W} \stackrel{\cup \Omega}{\longrightarrow} H^{\bullet}(A / / T, \mathbb{Q})^{a} .
$$

We compute the quantum period of $X$ by computing the $J$-function of $F=A / / G$ twisted [16] by the Euler class and the bundle $\mathcal{V}_{G}$, using the abelian/non-abelian correspondence [9]. An alternative method of calculation has been given by Andrew Strangeway [69].

We first compute the $J$-function of $A / / T$ twisted by the Euler class and the bundle $\mathcal{V}_{T}$. As in the proof of Theorem F.1, consider the bundles $\mathcal{V}_{T}$ and $\mathcal{V}_{G}$ equipped with the canonical $\mathbb{C}^{\times}$-action that rotates fibres and acts trivially on the base, and consider the twisted $J$-function $J_{\boldsymbol{e}, \mathcal{V}_{T}}$ of $A / / T . J_{\boldsymbol{e}, \mathcal{V}_{T}}$ was defined in (6) above, and is the restriction to the locus $\tau \in H^{0}(A / / T) \oplus H^{2}(A / / T)$ of what was denoted by $J_{\mathcal{V}_{T}}^{S \times \mathbb{C}^{\times}}(\tau)$ in [9]. The toric variety $A / / T=\mathbb{P}^{3} \times \mathbb{P}^{3} \times \mathbb{P}^{3}$ is Fano and Theorem C.1 gives

$$
J_{A / / T}(\tau)=e^{\tau / z} \sum_{l_{1}=0}^{\infty} \sum_{l_{2}=0}^{\infty} \sum_{l_{3}=0}^{\infty} \frac{Q_{1}^{l_{1}} Q_{2}^{l_{2}} Q_{3}^{l_{3}} e^{l_{1} \tau_{1}} e^{l_{2} \tau_{2}} e^{l_{3} \tau_{3}}}{\prod_{j=1}^{3} \prod_{k=1}^{l_{j}}\left(p_{j}+k z\right)^{4}},
$$

where $\tau=\tau_{1} p_{1}+\tau_{2} p_{2}+\tau_{3} p_{3}$ and we have identified the group ring $\mathbb{Q}\left[H_{2}(A / / T ; \mathbb{Z})\right]$ with $\mathbb{Q}\left[Q_{1}, Q_{2}, Q_{3}\right]$ via the $\mathbb{Q}$-linear map that sends $Q^{\beta}$ to $Q_{1}^{\left\langle\beta, p_{1}\right\rangle} Q_{2}^{\left\langle\beta, p_{2}\right\rangle} Q_{3}^{\left\langle\beta, p_{3}\right\rangle}$. Each line bundle summand in $\mathcal{V}_{T}$ is nef and $c_{1}(A / / T)-c_{1}\left(\mathcal{V}_{T}\right)$ is ample, so Theorem D.3 gives

$$
\begin{aligned}
& J_{\boldsymbol{e}, \mathcal{V}_{T}}(\tau)=e^{-\left(Q_{1} e^{\tau_{1}}+Q_{2} e^{\tau_{2}}+Q_{3} e^{\tau_{3}}\right) / z} e^{\tau / z} \\
& Q_{1}^{l_{1}} Q_{2}^{l_{2}} Q_{3}^{l_{3}} e^{l_{1} \tau_{1}} e^{l_{2} \tau_{2}} e^{l_{3} \tau_{3}} \\
& \times \sum_{l_{1}=0}^{\infty} \sum_{l_{2}=0}^{\infty} \sum_{l_{3}=0}^{\infty} \frac{\times \prod_{1 \leq i<j \leq 3} \prod_{k=1}^{l_{i}+l_{j}}\left(\lambda+p_{i}+p_{j}+k z\right)}{\prod_{j=1}^{3} \prod_{k=1}^{l_{j}}\left(p_{j}+k z\right)^{4}} \\
& l_{1}+l_{2}+l_{3} \\
& \times \prod_{k=1}\left(\lambda+p_{1}+p_{2}+p_{3}+k z\right) \text {. } \\
& k=1
\end{aligned}
$$

Consider now $F=A / / G=\operatorname{Gr}(2,4) \times \mathbb{P}^{3}$ and a point $t \in H^{\bullet}(F)$. Let $\epsilon_{1} \in H^{2}(F ; \mathbb{Q})$ be the pullback to $F$ (under projection to the first factor) of the ample generator 
of $H^{2}(\mathrm{Gr}(2,4))$ and let $\epsilon_{2} \in H^{2}(F ; \mathbb{Q})$ be the pullback to $F$ (under projection to the second factor) of the ample generator of $H^{2}\left(\mathbb{P}^{3}\right)$. Identify the group ring $\mathbb{Q}\left[H_{2}(F ; \mathbb{Z})\right]$ with $\mathbb{Q}\left[q_{1}, q_{2}\right]$ via the $\mathbb{Q}$-linear map which sends $Q^{\beta}$ to $q_{1}^{\left\langle\beta, \epsilon_{1}\right\rangle} q_{2}^{\left\langle\beta, \epsilon_{2}\right\rangle}$. In [9, Section 6.1] the authors consider the lift $\tilde{J}_{\mathcal{V}_{G} \times \mathbb{C}^{\times}}(t)$ of their twisted $J$-function $J_{\mathcal{V}_{G}}^{S \times \mathbb{C}^{\times}}(t)$ determined by a choice of lift $H^{\bullet}(A / / G ; \mathbb{Q}) \rightarrow H^{\bullet}(A / / T, \mathbb{Q})^{W}$. We restrict to the locus $t \in H^{0}(A / / G ; \mathbb{Q}) \oplus H^{2}(A / / G ; \mathbb{Q})$, considering the lift

$$
\tilde{J}_{\boldsymbol{e}, \mathcal{V}_{G}}(t):=\tilde{J}_{\mathcal{V}_{G} \times \mathbb{C}^{\times}}(t), \quad t \in H^{0}(A / / G ; \mathbb{Q}) \oplus H^{2}(A / / G ; \mathbb{Q}),
$$

of our twisted $J$-function $J_{\boldsymbol{e}}, \mathcal{V}_{G}$ determined by our choice of lift

$$
H^{\bullet}(A / / G ; \mathbb{Q}) \rightarrow H^{\bullet}(A / / T, \mathbb{Q})^{W} .
$$

Theorems 4.1.1 and 6.1.2 in [9] imply that

$$
\tilde{J}_{\boldsymbol{e}, \mathcal{V}_{G}}(\theta(t)) \cup \Omega=\left[\left(z \frac{\partial}{\partial \tau_{2}}-z \frac{\partial}{\partial \tau_{1}}\right) J_{\boldsymbol{e}, \mathcal{V}_{T}}(\tau)\right]_{\tau=t, Q_{1}=Q_{2}=-q_{1}, Q_{3}=q_{2}}
$$

for some ${ }^{12}$ function $\theta: H^{2}(A / / G ; \mathbb{Q}) \rightarrow H^{\bullet}\left(A / / G ; \Lambda_{A / / G}\right)$ such that $\theta(0)$ is in $H^{0}(A / / G ; \mathbb{Q}) \otimes \Lambda_{A / / G}$. Setting $t=0$ gives

$$
\begin{aligned}
& \tilde{J}_{\boldsymbol{e}, \mathcal{V}_{G}}(\theta(0)) \cup \Omega \\
& =e^{-\left(2 q_{1}+q_{2}\right) / z} \sum_{l_{1}=0}^{\infty} \sum_{l_{2}=0}^{\infty} \sum_{l_{3}=0}^{\infty} \frac{(-1)^{l_{1}+l_{2}} q_{1}^{l_{1}+l_{2}} q_{2}^{l_{3}}}{\times \prod_{1 \leq i<j \leq 3} \prod_{k=1}^{l_{i}+l_{j}}\left(\lambda+p_{i}+p_{j}+k z\right)} \\
& \prod_{j=1}^{3} \prod_{k=1}^{l_{j}}\left(p_{j}+k z\right)^{4} \\
& \quad \times\left(\prod_{k=1}^{l_{1}+l_{2}+l_{3}}\left(\lambda+p_{1}+p_{2}+p_{3}+k z\right)\right)\left(p_{2}-p_{1}+\left(l_{2}-l_{1}\right) z\right) .
\end{aligned}
$$

The left-hand side here takes the form

$$
\left(p_{2}-p_{1}\right)\left(1+\theta(0) z^{-1}+O\left(z^{-2}\right)\right)
$$

whereas the right-hand side is

$$
\left(p_{2}-p_{1}\right)\left(1-q_{1} z^{-1}+O\left(z^{-2}\right)\right) \text {. }
$$

${ }^{12}$ As in Theorem F.1, the map $\theta$ is grading-preserving and satisfies $\theta \equiv$ id modulo $q_{1}, q_{2}$. We will need only that $\theta(0) \in H^{0}(A / / G ; \mathbb{Q}) \otimes \Lambda_{A / / G}$, however, and we will see this explicitly below. 
We conclude that $\theta(0)=-q_{1}$ and hence, via the string equation, that

$$
\begin{aligned}
& \tilde{J}_{\boldsymbol{e}, \mathcal{V}_{G}}(0) \cup \Omega \\
& =e^{-\left(q_{1}+q_{2}\right) / z} \sum_{l_{1}=0}^{\infty} \sum_{l_{2}=0}^{\infty} \sum_{l_{3}=0}^{\infty} \frac{(-1)^{l_{1}+l_{2}} q_{1}^{l_{1}+l_{2}} q_{2}^{l_{3}}}{\times \prod_{1 \leq i<j \leq 3} \prod_{k=1}^{l_{i}+l_{j}}\left(\lambda+p_{i}+p_{j}\right.} \\
& \prod_{j=1}^{3} \prod_{k=1}^{l_{j}}\left(p_{j}+k z\right)^{4} \\
& \quad \times\left(\prod_{k=1}^{l_{1}+l_{2}+l_{3}}\left(\lambda+p_{1}+p_{2}+p_{3}+k z\right)\right)\left(p_{2}-p_{1}+\left(l_{2}-l_{1}\right) z\right) .
\end{aligned}
$$

We saw in Example D.8 how to extract the quantum period $G_{X}$ from the twisted $J$-function $J_{\boldsymbol{e}, \mathcal{V}_{G}}(0)$ : we take the non-equivariant limit $\lambda \rightarrow 0$, extract the component along the unit class $1 \in H^{\bullet}(A / / G ; \mathbb{Q})$, set $z=1$ and set $Q^{\beta}=t^{\left\langle\beta,-K_{X}\right\rangle}$. Thus we consider the right-hand side of (20), take the non-equivariant limit, extract the coefficient of $\Omega$ and set $z=1$ and $q_{1}=q_{2}=t$. This yields

$$
\begin{array}{r}
G_{X}(t)=e^{-2 t} \sum_{l_{1}=0}^{\infty} \sum_{l_{2}=0}^{\infty} \sum_{l_{3}=0}^{\infty}(-1)^{l_{1}+l_{2}} t^{l_{1}+l_{2}+l_{3}} \frac{\left(l_{1}+l_{2}\right) !\left(l_{1}+l_{3}\right) !\left(l_{2}+l_{3}\right) !\left(l_{1}+l_{2}+l_{3}\right) !}{\left(l_{1} !\right)^{4}\left(l_{2} !\right)^{4}\left(l_{3} !\right)^{4}} \\
\times\left(1+\left(l_{2}-l_{1}\right)\left(H_{l_{2}+l_{3}}-4 H_{l_{2}}\right)\right),
\end{array}
$$

where $H_{k}$ is the $k^{\text {th }}$ harmonic number. Regularizing gives

$$
\begin{aligned}
\widehat{G}_{X}(t)=1+10 t^{2}+42 t^{3}+414 t^{4}+ & 3300 t^{5}+29890 t^{6} \\
& +275940 t^{7}+2608270 t^{8}+25305000 t^{9}+\cdots .
\end{aligned}
$$

Minkowski period sequence 101

\section{The Fano manifold $\mathrm{MM}_{2-18}$}

Mori-Mukai construction A double cover of $\mathbb{P}^{1} \times \mathbb{P}^{2}$ with branch locus a divisor of bidegree $(2,2)$.

Our construction A member $X$ of $|2 L+2 M|$ in the toric variety $F$ with weight data

\begin{tabular}{ccccccc}
$x_{0}$ & $x_{1}$ & $x_{2}$ & $y_{0}$ & $y_{1}$ & $w$ & \\
\cline { 1 - 5 } & 1 & 1 & 0 & 0 & 1 & $L$ \\
0 & 0 & 0 & 1 & 1 & 1 & $M$
\end{tabular}

and Nef $F=\langle L, L+M\rangle$. We have:

- $-K_{F}=4 L+3 M$ is ample, that is $F$ is a Fano variety. 
- $\quad X \sim 2 L+2 M$ is nef.

- $-\left(K_{F}+X\right) \sim 2 L+M$ is ample.

The two constructions coincide The defining equation of $X$ is

$$
w^{2}=f_{2,2}\left(x_{0}, x_{1}, x_{2} ; y_{0}, y_{1}\right)
$$

and so the morphism $X \rightarrow \mathbb{P}^{2} \times \mathbb{P}^{1}$ which sends the point $\left[x_{0}: x_{1}: x_{2}: y_{0}: y_{1}: w\right]$ of $X$ to the point $\left[x_{0}: x_{1}: x_{2}: y_{0}: y_{1}\right]$ of $\mathbb{P}^{2} \times \mathbb{P}^{1}$ exhibits $X$ as a double cover of $\mathbb{P}^{2} \times \mathbb{P}^{1}$ branched over a divisor of bidegree $(2,2)$.

The quantum period Corollary D.5 yields

$$
G_{X}(t)=e^{-2 t} \sum_{l=0}^{\infty} \sum_{m=0}^{\infty} t^{2 l+m} \frac{(2 l+2 m) !}{(l !)^{3}(m !)^{2}(l+m) !}
$$

and regularizing gives

$$
\begin{aligned}
\widehat{G}_{X}(t)=1+6 t^{2}+48 t^{3}+282 t^{4}+ & 2400 t^{5}+22020 t^{6} \\
& +184800 t^{7}+1684410 t^{8}+15798720 t^{9}+\cdots .
\end{aligned}
$$

Minkowski period sequence 74

\section{The Fano manifold $M_{2-19}$}

Mori-Mukai construction The blow-up of $B_{4} \subset \mathbb{P}^{5}$ with centre a line on it.

Our construction A codimension-2 complete intersection $X$ of type

$$
(L+M) \cap(L+M)
$$

in the toric variety $F$ with weight data

\begin{tabular}{cccccccc}
$s_{0}$ & $s_{1}$ & $s_{2}$ & $s_{3}$ & $x$ & $x_{4}$ & $x_{5}$ & \\
\cline { 1 - 6 } 1 & 1 & 1 & 1 & -1 & 0 & 0 & $L$ \\
0 & 0 & 0 & 0 & 1 & 1 & 1 & $M$
\end{tabular}

and Nef $F=\langle L, M\rangle$. We have:

- $-K_{F}=3 L+3 M$ is ample, that is, $F$ is a Fano variety.

- $X$ is the complete intersection of two ample divisors on $F$.

- $-\left(K_{F}+\Lambda\right) \sim L+M$ is ample. 
The two constructions coincide The morphism $F \rightarrow \mathbb{P}^{5}$ that sends (contravariantly) the homogeneous co-ordinate functions $\left[x_{0}, \ldots, x_{5}\right]$ to $\left[x s_{0}, \ldots, x s_{3}, x_{4}, x_{5}\right]$ blows up the line $\left(x_{0}=\cdots=x_{3}=0\right)$ in $\mathbb{P}^{5}$. Now take the proper transform of a $B_{4}$ containing this line.

The quantum period Corollary D.5 yields

and regularizing gives

$$
G_{X}(t)=e^{-t} \sum_{l=0}^{\infty} \sum_{m=l}^{\infty} t^{l+m} \frac{(l+m) !(l+m) !}{(l !)^{4}(m-l) !(m !)^{2}}
$$

$$
\begin{aligned}
\widehat{G}_{X}(t)=1+8 t^{2}+30 t^{3}+240 t^{4}+1920 t^{5}+13490 t^{6} \\
+121800 t^{7}+953680 t^{8}+8465520 t^{9}+\cdots .
\end{aligned}
$$

Minkowski period sequence 86

\section{The Fano manifold $\mathbf{M M}_{2-20}$}

Mori-Mukai construction The blow-up of $B_{5} \subset \mathbb{P}^{6}$ with centre a twisted cubic on it.

Our construction The vanishing locus $X$ of a general section of the vector bundle

$$
E=\left(S^{\star} \otimes \mathcal{O}_{\mathbb{P}^{2}}(1)\right) \oplus\left(\operatorname{det} S^{\star} \otimes \mathcal{O}_{\mathbb{P}^{2}}\right)^{\oplus 3}
$$

on the key variety $F=\operatorname{Gr}(2,5) \times \mathbb{P}^{2}$, where $S$ is the universal bundle of subspaces on $\operatorname{Gr}(2,5)$.

The two constructions coincide Consider $\mathbb{C}^{5}$ with basis $e_{0}, \ldots, e_{4}$. Let $M(2,5)^{\times}$ denote the space of $2 \times 5$ complex matrices of full rank. As is customary we represent a point $W$ in $\operatorname{Gr}\left(2, \mathbb{C}^{5}\right)$ by a matrix

$$
\left(\begin{array}{lllll}
a_{0} & a_{1} & a_{2} & a_{3} & a_{4} \\
b_{0} & b_{1} & b_{2} & b_{3} & b_{4}
\end{array}\right) \in M(2,5)^{\times}
$$

up to the action of $\mathrm{GL}_{2}(\mathbb{C})$ from the left. A basis element $e_{i}$ of $\mathbb{C}^{5}$ gives a section of the rank-2 vector bundle $S^{\star}$ that evaluates as

$$
e_{i}(W)=\left(\begin{array}{l}
a_{i} \\
b_{i}
\end{array}\right)
$$

Consider now the section

$$
s=e_{0} x_{0}+e_{1} x_{1}+e_{2} x_{2} \in \Gamma\left(\operatorname{Gr}(2,5) \times \mathbb{P}^{2} ; S^{\star} \otimes \mathcal{O}(1)\right) .
$$


Let $Y \subset \operatorname{Gr}(2,5) \times \mathbb{P}^{2}$ be the vanishing locus of $s$ and let $p: Y \rightarrow \operatorname{Gr}(2,5)$ be the projection. $Y$ consists of pairs $(W, x) \in M(2,5)^{\times} \times \mathbb{P}^{2}$ such that $x=\left(x_{0}, x_{1}, x_{2}\right)$ is a solution of the system

$$
W \cdot\left(\begin{array}{c}
x_{0} \\
x_{1} \\
x_{2} \\
0 \\
0
\end{array}\right)=0
$$

that is, $p: Y \rightarrow \operatorname{Gr}(2,5)$ blows up the locus $Z \subset \operatorname{Gr}(2,5)$ consisting of those $W$ such that

$$
\operatorname{rk}\left(\begin{array}{lll}
a_{0} & a_{1} & a_{2} \\
b_{0} & b_{1} & b_{2}
\end{array}\right)<2
$$

In Plücker co-ordinates

$$
x_{i j}=\operatorname{det}\left(\begin{array}{ll}
a_{i} & a_{j} \\
b_{i} & b_{j}
\end{array}\right)
$$

this is the locus where $x_{01}=x_{02}=x_{12}=0$. Thus, $Z$ is the cubic scroll defined by

$$
x_{01}=x_{02}=x_{12}=0 \quad \text { and } \quad \operatorname{rk}\left(\begin{array}{lll}
x_{03} & x_{13} & x_{14} \\
x_{04} & x_{14} & x_{24}
\end{array}\right)<2 .
$$

Intersecting with 3 more hyperplane sections in the Plücker embedding, we get the blow-up of $B_{5}$ along a twisted cubic.

Abelianization Consider $\operatorname{Gr}(2,5)$ as the geometric quotient $\mathbb{C}^{10} / / \mathrm{GL}_{2}(\mathbb{C})$, where we regard $\mathbb{C}^{10}$ as the space $M(2,5)$ of $2 \times 5$ complex matrices and $\mathrm{GL}_{2}(\mathbb{C})$ acts by multiplication on the left. The universal bundle $S$ of subspaces on $\operatorname{Gr}(2,5)$ is the bundle on $\mathbb{C}^{10} / / \mathrm{GL}_{2}(\mathbb{C})$ determined by $V_{\text {std }}^{\star}$, where $V_{\text {std }}$ is the standard representation of $\mathrm{GL}_{5}(\mathbb{C})$. Consider the situation as in $[9$, Section 3.1] with

- the space that is denoted by $X$ in [9] set equal to $A=\mathbb{C}^{13}$, regarded as the space of pairs

$$
\left\{(M, w) \mid M \text { is a } 2 \times 5 \text { complex matrix and } w \in \mathbb{C}^{3} \text { is a vector }\right\} ;
$$

- $G=\mathrm{GL}_{2}(\mathbb{C}) \times \mathbb{C}^{\times}$, acting on $A$ as

$$
(g, \lambda):(M, w) \mapsto(g M, \lambda w) ;
$$

- $T=\left(\mathbb{C}^{\times}\right)^{3}$, the diagonal subtorus in $G$;

- the group that is denoted by $S$ in [9] set equal to the trivial group; 
- $\mathcal{V}$ equal to the representation of $G$ given by

$$
\left(V_{\text {std }} \otimes V_{\text {std }}\right) \oplus\left(\operatorname{det} V_{\text {std }} \otimes V_{\text {triv }}\right)^{\oplus 3} \text {, }
$$

where $V_{\text {triv }}$ is the trivial 1 -dimensional representation of $\mathbb{C}^{\times}$.

It is clear that $A / / G=F$, whereas $A / / T=\mathbb{P}^{4} \times \mathbb{P}^{4} \times \mathbb{P}^{2}$. The Weyl group $W=\mathbb{Z} / 2 \mathbb{Z}$ permutes the first and second factors of the product $\mathbb{P}^{4} \times \mathbb{P}^{4} \times \mathbb{P}^{2}$. The representation $\mathcal{V}$ induces the vector bundle $\mathcal{V}_{G}=E$ over $F$, whereas the representation $\mathcal{V}$ induces the vector bundle

$$
\mathcal{V}_{T}=\mathcal{O}(1,0,1) \oplus \mathcal{O}(0,1,1) \oplus \mathcal{O}(1,1,0)^{\oplus 3}
$$

over $A / / T$.

The abelian/non-abelian correspondence We proceed exactly as for $\mathrm{MM}_{2-17}$, replacing:

- $\mathbb{P}^{3} \times \mathbb{P}^{3} \times \mathbb{P}^{3}$ by $\mathbb{P}^{4} \times \mathbb{P}^{4} \times \mathbb{P}^{2}$, throughout.

- Equation (16) by

$$
\begin{aligned}
& J_{A / / T}(\tau) \\
& =e^{\tau / z} \sum_{l_{1}=0}^{\infty} \sum_{l_{2}=0}^{\infty} \sum_{l_{3}=0}^{\infty} \frac{Q_{1}^{l_{1}} Q_{2}^{l_{2}} Q_{3}^{l_{3}} e^{l_{1} \tau_{1}} e^{l_{2} \tau_{2}} e^{l_{3} \tau_{3}}}{\prod_{k=1}^{l_{1}}\left(p_{1}+k z\right)^{5} \prod_{k=1}^{l_{2}}\left(p_{2}+k z\right)^{5} \prod_{k=1}^{l_{3}}\left(p_{3}+k z\right)^{3}}
\end{aligned}
$$

- Equation (17) by

$$
\begin{aligned}
J_{\boldsymbol{e}, \mathcal{V}_{T}}(\tau)=e^{-\left(Q_{1} e^{\tau_{1}}+Q_{2} e^{\tau_{2}}+Q_{3} e^{\tau_{3}}\right) / z} e^{\tau / z} & \\
& \times \sum_{l_{1}=0}^{\infty} \sum_{l_{2}=0}^{\infty} \sum_{l_{3}=0}^{\infty} \frac{Q_{1}^{l_{1}} Q_{2}^{l_{2}} Q_{3}^{l_{3}} e^{l_{1} \tau_{1}} e^{l_{2} \tau_{2}} e^{l_{3} \tau_{3}}}{\times \prod_{k=1}^{l_{1}+l_{2}}\left(\lambda+p_{1}+p_{2}+k z\right)^{3}} \\
& \times \prod_{k=1}^{l_{1} l_{1}\left(p_{1}+k z\right)^{5} \prod_{k=1}^{l_{2}}\left(p_{2}+k z\right)^{5}} \times \prod_{k=1}^{l_{3}}\left(p_{3}+k z\right)^{3} \\
& \left.\times p_{1}+p_{3}+k z\right) \prod_{k=1}^{l_{2}+l_{3}}\left(\lambda+p_{2}+p_{3}+k z\right) .
\end{aligned}
$$

- $\operatorname{Gr}(2,4) \times \mathbb{P}^{3}$ by $\operatorname{Gr}(2,5) \times \mathbb{P}^{2}$, throughout. 
- Equation (18) by

$$
\begin{aligned}
& \tilde{J}_{\boldsymbol{e}, \mathcal{V}_{G}}(\theta(0)) \cup \Omega e^{-\left(2 q_{1}+q_{2}\right) / z} \sum_{l_{1}=0}^{\infty} \sum_{l_{2}=0}^{\infty} \sum_{l_{3}=0}^{\infty} \frac{(-1)^{l_{1}+l_{2}} q_{1}^{l_{1}+l_{2}} q_{2}^{l_{3}}}{\prod_{k=1}^{l_{1}}\left(p_{1}+k z\right)^{5} \prod_{k=1}^{l_{2}}\left(p_{2}+k z\right)^{5}} \\
& \times \prod_{k=1}^{l_{3}}\left(p_{3}+k z\right)^{3}
\end{aligned}
$$

- Equation (19) by

$$
\left(p_{2}-p_{1}\right)\left(1+O\left(z^{-2}\right)\right) \text {. }
$$

- The conclusion $\theta(0)=-q_{1}$ by $\theta(0)=0$ and (20) by

$$
\begin{aligned}
& \tilde{J}_{\boldsymbol{e}, \mathcal{V}_{G}}(0) \cup \Omega \\
& =e^{-\left(2 q_{1}+q_{2}\right) / z} \sum_{l_{1}=0}^{\infty} \sum_{l_{2}=0}^{\infty} \sum_{l_{3}=0}^{\infty} \frac{(-1)^{l_{1}+l_{2}} q_{1}^{l_{1}+l_{2}} q_{2}^{l_{3}}}{\prod_{k=1}^{l_{1}}\left(p_{1}+k z\right)^{5} \prod_{k=1}^{l_{2}}\left(p_{2}+k z\right)^{5}} \\
& \times \prod_{k=1}^{l_{3}}\left(p_{3}+k z\right)^{3} \\
& \quad \times \prod_{k=1}^{l_{1}+l_{3}}\left(\lambda+p_{1}+p_{3}+k z\right) \prod_{k=1}^{l_{2}+l_{3}}\left(\lambda+p_{2}+p_{3}+k z\right)\left(p_{2}-p_{1}+\left(l_{2}-l_{1}\right) z\right) .
\end{aligned}
$$

This yields

$$
\begin{array}{r}
G_{X}(t)=e^{-3 t} \sum_{l_{1}=0}^{\infty} \sum_{l_{2}=0}^{\infty} \sum_{l_{3}=0}^{\infty}(-1)^{l_{1}+l_{2}} t^{l_{1}+l_{2}+l_{3}} \frac{\left(\left(l_{1}+l_{2}\right) !\right)^{3}\left(l_{1}+l_{3}\right) !\left(l_{2}+l_{3}\right) !}{\left(l_{1} !\right)^{5}\left(l_{2} !\right)^{5}\left(l_{3} !\right)^{3}} \\
\times\left(1+\left(l_{2}-l_{1}\right)\left(H_{l_{2}+l_{3}}-5 H_{l_{2}}\right)\right),
\end{array}
$$

where $H_{k}$ is the $k^{\text {th }}$ harmonic number. Regularizing gives

$$
\begin{aligned}
\widehat{G}_{X}(t)=1+8 t^{2}+36 t^{3}+288 t^{4}+ & 2220 t^{5}+18260 t^{6} \\
& +154560 t^{7}+1348480 t^{8}+11977560 t^{9}+\cdots .
\end{aligned}
$$

Minkowski period sequence 87 


\section{The Fano manifold $M_{2-21}$}

Mori-Mukai construction The blow-up of a quadric 3 -fold $Q \subset \mathbb{P}^{4}$ with centre a rational normal curve of degree 4 on it.

Our construction The vanishing locus $X$ of a general section of the vector bundle

$$
E=\left(S^{\star} \otimes \mathcal{O}_{\mathbb{P}^{4}}(1)\right)^{\oplus 2} \oplus\left(\operatorname{det} S^{\star} \otimes \mathcal{O}_{\mathbb{P}^{4}}\right)
$$

on the key variety $F=\operatorname{Gr}(2,4) \times \mathbb{P}^{4}$, where $S$ is the universal bundle of subspaces on $\operatorname{Gr}(2,4)$.

The two constructions coincide Consider $\mathbb{C}^{4}$ with basis $e_{0}, \ldots, e_{3}$. Let $M(2,4)^{\times}$ denote the space of $2 \times 4$ complex matrices of full rank and represent a point $W$ in $\operatorname{Gr}\left(2, \mathbb{C}^{4}\right)$ by

$$
W=\left(\begin{array}{llll}
a_{0} & a_{1} & a_{2} & a_{3} \\
b_{0} & b_{1} & b_{2} & b_{3}
\end{array}\right) \in M(2,4)^{\times}
$$

up to the action of $\mathrm{GL}_{2}(\mathbb{C})$ from the left. A basis element $e_{i}, 0 \leq i \leq 3$, of $\mathbb{C}^{4}$ gives a section of the rank-2 vector bundle $S^{\star}$ that evaluates as

$$
e_{i}(W)=\left(\begin{array}{l}
a_{i} \\
b_{i}
\end{array}\right) \text {. }
$$

Let $x_{0}, \ldots, x_{4}$ be homogeneous co-ordinates on $\mathbb{P}^{4}$ and consider the two sections

$$
s_{1}=e_{0} x_{0}+e_{1} x_{1}+e_{2} x_{2}+e_{3} x_{3} \quad \text { and } s_{2}=e_{0} x_{1}+e_{1} x_{2}+e_{2} x_{3}+e_{3} x_{4}
$$

in $\Gamma\left(\operatorname{Gr}(2,4) \times \mathbb{P}^{4} ; S^{\star} \otimes \mathcal{O}(1)\right)$. Let $Y \subset \operatorname{Gr}(2,4) \times \mathbb{P}^{4}$ denote the locus on which $s_{1}$ and $s_{2}$ both vanish, and let $p: Y \rightarrow \operatorname{Gr}(2,4)$ and $q: Y \rightarrow \mathbb{P}^{4}$ denote the projections to the two factors of $\operatorname{Gr}(2,4) \times \mathbb{P}^{4}$. The locus $Y$ consists of pairs $(W, x) \in M(2,4)^{\times} \times \mathbb{P}^{4}$ such that

$$
W \subset \operatorname{Ker}\left(\begin{array}{ll}
x_{0} & x_{1} \\
x_{1} & x_{2} \\
x_{2} & x_{3} \\
x_{3} & x_{4}
\end{array}\right) \text {. }
$$

It follows that $q: Y \rightarrow \mathbb{P}^{4}$ blows up the locus $Z$ given by the condition

$$
\operatorname{rk}\left(\begin{array}{llll}
x_{0} & x_{1} & x_{2} & x_{3} \\
x_{1} & x_{2} & x_{3} & x_{4}
\end{array}\right)<2
$$

that is, the rational normal curve. Intersecting with $p^{\star}(H)$, where $H \in\left|\operatorname{det} S^{\star}\right|$, gives the proper transform of a quadric 3 -fold containing $Z$. 
Abelianization Consider $\operatorname{Gr}(2,4)$ as the geometric quotient $\mathbb{C}^{8} / / \mathrm{GL}_{2}(\mathbb{C})$, where we regard $\mathbb{C}^{8}$ as the space $M(2,4) \times$ of $2 \times 4$ complex matrices and $\mathrm{GL}_{2}(\mathbb{C})$ acts by multiplication on the left. The universal bundle $S$ of subspaces on $\operatorname{Gr}(2,4)$ is the bundle on $\mathbb{C}^{8} / / \mathrm{GL}_{2}(\mathbb{C})$ determined by $V_{\text {std }}^{\star}$, where $V_{\text {std }}$ is the standard representation of $\mathrm{GL}_{2}(\mathbb{C})$. Consider the situation as in $[9$, Section 3.1] with

- the space that is denoted by $X$ in [9] set equal to $A=\mathbb{C}^{13}$, regarded as the space of pairs

$\left\{(M, w) \mid M\right.$ is a $2 \times 4$ complex matrix and $w \in \mathbb{C}^{5}$ is a vector $\} ;$

- $G=\mathrm{GL}_{2}(\mathbb{C}) \times \mathbb{C}^{\times}$, acting on $A$ as

$$
(g, \lambda):(M, w) \mapsto(g M, \lambda w) ;
$$

- $T=\left(\mathbb{C}^{\times}\right)^{3}$, the diagonal subtorus in $G$;

- the group that is denoted by $S$ in [9] set equal to the trivial group;

- $\mathcal{V}$ equal to the representation of $G=\mathrm{GL}_{2}(\mathbb{C}) \times \mathbb{C}^{\times}$given by

$$
\left(V_{\text {std }} \otimes V_{\text {std }}\right)^{\oplus 2} \oplus\left(\operatorname{det} V_{\text {std }} \otimes V_{\text {triv }}\right),
$$

where $V_{\text {triv }}$ is the trivial 1 -dimensional representation of $\mathbb{C}^{\times}$.

It is clear that $A / / G=F$, whereas $A / / T=\mathbb{P}^{3} \times \mathbb{P}^{3} \times \mathbb{P}^{4}$. The Weyl group $W=\mathbb{Z} / 2 \mathbb{Z}$ permutes the first and second factors of the product $\mathbb{P}^{3} \times \mathbb{P}^{3} \times \mathbb{P}^{4}$. The representation $\mathcal{V}$ induces the vector bundle $\mathcal{V}_{G}=E$ over $F$, whereas the representation $\mathcal{V}$ induces the vector bundle

$$
\mathcal{V}_{T}=\mathcal{O}(1,0,1)^{\oplus 2} \oplus \mathcal{O}(0,1,1)^{\oplus 2} \oplus \mathcal{O}(1,1,0)
$$

over $A / / T=\mathbb{P}^{3} \times \mathbb{P}^{3} \times \mathbb{P}^{4}$.

The abelian/non-abelian correspondence Again we proceed as for $\mathrm{MM}_{2-17}$, replacing:

- $\mathbb{P}^{3} \times \mathbb{P}^{3} \times \mathbb{P}^{3}$ by $\mathbb{P}^{3} \times \mathbb{P}^{3} \times \mathbb{P}^{4}$, throughout.

- Equation (16) by

$$
\begin{aligned}
& J_{A / / T}(\tau) \\
& =e^{\tau / z} \sum_{l_{1}=0}^{\infty} \sum_{l_{2}=0}^{\infty} \sum_{l_{3}=0}^{\infty} \frac{Q_{1}^{l_{1}} Q_{2}^{l_{2}} Q_{3}^{l_{3}} e^{l_{1} \tau_{1}} e^{l_{2} \tau_{2}} e^{l_{3} \tau_{3}}}{\prod_{k=1}^{l_{1}}\left(p_{1}+k z\right)^{4} \prod_{k=1}^{l_{2}}\left(p_{2}+k z\right)^{4} \prod_{k=1}^{l_{3}}\left(p_{3}+k z\right)^{5}} .
\end{aligned}
$$


- Equation (17) by

$$
\begin{aligned}
J_{\boldsymbol{e}, \mathcal{V}_{T}}(\tau)=e^{-\left(Q_{1} e^{\tau_{1}}+Q_{2} e^{\tau_{2}}+Q_{3} e^{\tau_{3}}\right) / z} e^{\tau / z} & \\
& \times \sum_{l_{1}=0}^{\infty} \sum_{l_{2}=0}^{\infty} \sum_{l_{3}=0}^{\infty} \frac{Q_{1}^{l_{1}} Q_{2}^{l_{2}} Q_{3}^{l_{3}} e^{l_{1} \tau_{1}} e^{l_{2} \tau_{2}} e^{l_{3} \tau_{3}}}{\times \prod_{k=1}^{l_{1}+l_{2}}\left(\lambda+p_{1}+p_{2}+k z\right)} \\
& \times \prod_{k=1}^{l_{1}\left(p_{1}+k z\right)^{4} \prod_{k=1}^{l_{2}}\left(p_{2}+k z\right)^{4}} \times \quad \times \prod_{k=1}^{l_{3}}\left(p_{3}+k z\right)^{5} \\
& \\
& \times \prod_{k=1}^{l_{1}+l_{3}}\left(\lambda+p_{1}+p_{3}+k z\right)^{2} \prod_{k=1}^{l_{2}+l_{3}}\left(\lambda+p_{2}+p_{3}+k z\right)^{2} .
\end{aligned}
$$

- $\operatorname{Gr}(2,4) \times \mathbb{P}^{3}$ by $\operatorname{Gr}(2,4) \times \mathbb{P}^{4}$, throughout.

- Equation (18) by

$$
\begin{aligned}
& \tilde{J}_{\boldsymbol{e}, \mathcal{V}_{G}}(\theta(0)) \cup \Omega \\
& (-1)^{l_{1}+l_{2}} q_{1}^{l_{1}+l_{2}} q_{2}^{l_{3}} \\
& =e^{-\left(2 q_{1}+q_{2}\right) / z} \sum_{l_{1}=0}^{\infty} \sum_{l_{2}=0}^{\infty} \sum_{l_{3}=0}^{\infty} \frac{\times \prod_{k=1}^{l_{1}+l_{2}}\left(\lambda+p_{1}+p_{2}+k z\right)}{\prod_{k=1}^{l_{1}}\left(p_{1}+k z\right)^{4} \prod_{k=1}^{l_{2}}\left(p_{2}+k z\right)^{4}} \\
& \times \prod_{k=1}^{l_{3}}\left(p_{3}+k z\right)^{5} \\
& \times \prod_{k=1}^{l_{1}+l_{3}}\left(\lambda+p_{1}+p_{3}+k z\right)^{2} \prod_{k=1}^{l_{2}+l_{3}}\left(\lambda+p_{2}+p_{3}+k z\right)^{2}\left(p_{2}-p_{1}+\left(l_{2}-l_{1}\right) z\right) .
\end{aligned}
$$

- Equation (19) by

$$
\left(p_{2}-p_{1}\right)\left(1-2 q_{1} z^{-1}+O\left(z^{-2}\right)\right) .
$$

- The conclusion $\theta(0)=-q_{1}$ by $\theta(0)=-2 q_{1}$, and (20) by

$$
\begin{aligned}
& \tilde{J}_{\boldsymbol{e}, \mathcal{V}_{G}}(0) \cup \Omega \\
& \begin{array}{r}
=e^{-q_{2} / z} \sum_{l_{1}=0}^{\infty} \sum_{l_{2}=0}^{\infty} \sum_{l_{3}=0}^{\infty} \frac{(-1)^{l_{1}+l_{2}} q_{1}^{l_{1}+l_{2}} q_{2}^{l_{3}} \prod_{k=1}^{l_{1}+l_{2}}\left(\lambda+p_{1}+p_{2}+k z\right)}{\prod_{k=1}^{l_{1}}\left(p_{1}+k z\right)^{4} \prod_{k=1}^{l_{2}}\left(p_{2}+k z\right)^{4}} \\
\times \prod_{k=1}^{l_{3}}\left(p_{3}+k z\right)^{5}
\end{array} \\
& \times \prod_{k=1}^{l_{1}+l_{3}}\left(\lambda+p_{1}+p_{3}+k z\right)^{2} \\
& \times \prod_{k=1}^{l_{2}+l_{3}}\left(\lambda+p_{2}+p_{3}+k z\right)^{2}\left(p_{2}-p_{1}+\left(l_{2}-l_{1}\right) z\right) .
\end{aligned}
$$


This yields

$$
\begin{array}{r}
G_{X}(t)=e^{-t} \sum_{l_{1}=0}^{\infty} \sum_{l_{2}=0}^{\infty} \sum_{l_{3}=0}^{\infty}(-1)^{l_{1}+l_{2}} t^{l_{1}+l_{2}+l_{3}} \frac{\left(l_{1}+l_{2}\right) !\left(\left(l_{1}+l_{3}\right) !\right)^{2}\left(\left(l_{2}+l_{3}\right) !\right)^{2}}{\left(l_{1} !\right)^{4}\left(l_{2} !\right)^{4}\left(l_{3} !\right)^{5}} \\
\times\left(1+\left(l_{2}-l_{1}\right)\left(2 H_{l_{2}+l_{3}}-4 H_{l_{2}}\right)\right),
\end{array}
$$

where $H_{k}$ is the $k^{\text {th }}$ harmonic number. Regularizing gives

$$
\begin{aligned}
\widehat{G}_{X}(t)=1+8 t^{2}+24 t^{3}+240 t^{4}+1440 t^{5} & +11960 t^{6} \\
& +89040 t^{7}+731920 t^{8}+5913600 t^{9}+\cdots .
\end{aligned}
$$

\section{Minkowski period sequence 84}

\section{The Fano manifold $\mathrm{MM}_{2-22}$}

Mori-Mukai construction The blow-up of $B_{5} \subset \mathbb{P}^{6}$ with centre a conic on it.

Our construction A complete intersection $X$ of type $L \cap M \cap M \cap M$ in the flag manifold $\mathrm{Fl}=\mathrm{Fl}\left(1,2 ; \mathbb{C}^{5}\right)$, where $p: \mathrm{Fl} \rightarrow \mathbb{P}^{4}$ and $q: \mathrm{Fl} \rightarrow \mathrm{Gr}=\mathrm{Gr}(2,5)$ are the natural projections, $L=p^{\star} \mathcal{O}(1), M=q^{\star} \operatorname{det} S^{\star}$ and $S$ is the universal bundle of subspaces on Gr.

The two constructions coincide Note that $\mathrm{Fl}=\mathbb{P}(S)$ is the projectivization of the universal bundle $S$ of subspaces on $\mathrm{Gr}$. On Fl we have a natural surjection of vector bundles:

$$
q^{\star} S^{\star} \rightarrow L \quad \text { inducing } \quad H^{0}\left(\mathrm{Fl}, q^{\star} S^{\star}\right) \cong H^{0}(\mathrm{Fl}, L)
$$

Let $s \in H^{0}(\mathrm{Fl}, L)$ be a general section and $Y$ be the locus $(s=0) \subset \mathrm{Fl}$. It is clear that $q: Y \rightarrow$ Gr blows up $Z=(\tilde{s}=0) \subset \mathrm{Gr}$, where $\tilde{s}$ "is" $s$, now thought of as an element of $H^{0}\left(\mathrm{Gr}, S^{\star}\right)$. We are done, as $Z=Z_{1,1}$ maps to a quadric under the Plücker embedding.

Abelianization Consider the situation as in [9, Section 3.1] with

- the space that is denoted by $X$ in [9] set equal to $A=\mathbb{C}^{12}$, regarded as the space of pairs

$$
\left\{(v, w) \mid v \in \mathbb{C}^{2} \text { is a row vector and } w \text { is a } 2 \times 5 \text { complex matrix }\right\} ;
$$

- $G=\mathbb{C}^{\times} \times \mathrm{GL}_{2}(\mathbb{C})$, acting on $A$ as

$$
(\lambda, g):(v, w) \mapsto\left(\lambda v g^{-1}, g w\right) ;
$$


- $T=\left(\mathbb{C}^{\times}\right)^{3}$, the diagonal subtorus in $G$;

- the group that is denoted by $S$ in [9] set equal to the trivial group;

- $\mathcal{V}$ equal to the representation of $G$ given by the direct sum of one copy of the standard representation of the first factor $\mathbb{C}^{\times}$and three copies of the determinant of the standard representation of the second factor $\mathrm{GL}_{2}(\mathbb{C})$.

Then $A / / G$ is the flag manifold $\mathrm{Fl}=\mathrm{Fl}\left(1,2 ; \mathbb{C}^{5}\right)$, whereas $A / / T$ is the toric variety with weight data

$\begin{array}{rrrrrrrrrrrrr}1 & 1 & 1 & 1 & 1 & 0 & 0 & 0 & 0 & 0 & -1 & 0 & L_{1} \\ 0 & 0 & 0 & 0 & 0 & 1 & 1 & 1 & 1 & 1 & 0 & -1 & L_{2} \\ 0 & 0 & 0 & 0 & 0 & 0 & 0 & 0 & 0 & 0 & 1 & 1 & H\end{array}$

and Nef $=\left\langle L_{1}, L_{2}, H\right\rangle$; that is, $A / / T$ is the projective bundle $\mathbb{P}(\mathcal{O}(-1,0) \oplus \mathcal{O}(0,-1))$ over $\mathbb{P}^{4} \times \mathbb{P}^{4}$. The non-trivial element of the Weyl group $W=\mathbb{Z} / 2 \mathbb{Z}$ exchanges the two factors of $\mathbb{P}^{4} \times \mathbb{P}^{4}$. The representation $\mathcal{V}$ induces the vector bundle $\mathcal{V}_{G}=L \oplus M^{\oplus 3}$ over $A / / G=\mathrm{Fl}$, whereas the representation $\mathcal{V}$ induces the vector bundle $\mathcal{V}_{T}=$ $H \oplus\left(L_{1}+L_{2}\right)^{\oplus 3}$ over $A / / T$.

The abelian/non-abelian correspondence Let $p_{1}, p_{2}, p_{3} \in H^{2}(A / / T ; \mathbb{Q})$ denote the first Chern classes of the line bundles $L_{1}, L_{2}$ and $H$, respectively. We fix a lift of $H^{\bullet}(A / / G ; \mathbb{Q})$ to $H^{\bullet}(A / / T, \mathbb{Q})^{W}$ in the sense of $[9$, Section 3]; there are many possible choices for such a lift and the precise choice made will be unimportant in what follows. The lift allows us to regard $H^{\bullet}(A / / G ; \mathbb{Q})$ as a subspace of $H^{\bullet}(A / / T, \mathbb{Q})^{W}$, which maps isomorphically to the Weyl-anti-invariant part $H^{\bullet}(A / / T, \mathbb{Q})^{a}$ of $H^{\bullet}(A / / T, \mathbb{Q})$ via

$$
H^{\bullet}(A / / T, \mathbb{Q})^{W} \stackrel{\cup\left(p_{2}-p_{1}\right)}{\longrightarrow} H^{\bullet}(A / / T, \mathbb{Q})^{a} .
$$

We compute the quantum period of $X$ by computing the $J$-function of $\mathrm{Fl}=A / / G$ twisted [16] by the Euler class and the bundle $\mathcal{V}_{G}$, using the abelian/non-abelian correspondence [9].

Our first step is to compute the $J$-function of $A / / T$ twisted by the Euler class and the bundle $\mathcal{V}_{T}$. As in Section D.1, and as in [9], consider the bundles $\mathcal{V}_{T}$ and $\mathcal{V}_{G}$ equipped with the canonical $\mathbb{C}^{\times}$-action that rotates fibres and acts trivially on the base. We will compute the twisted $J$-function $J_{\boldsymbol{e}, \mathcal{V}_{T}}$ of $A / / T$ using the quantum Lefschetz theorem; $J_{\boldsymbol{e}}, \nu_{T}$ was defined in (6) above and is the restriction to the locus $\tau \in H^{0}(A / / T) \oplus H^{2}(A / / T)$ of what was denoted by $J_{\mathcal{V}_{T} \times \mathbb{C}^{\times}}(\tau)$ in [9]. The toric 
variety $A / / T$ is Fano, so Theorem C.1 gives

$$
\begin{array}{r}
J_{A / / T}(\tau)=e^{\tau / z} \sum_{l, m, n \geq 0} \frac{Q_{1}^{l} Q_{2}^{m} Q_{3}^{n} e^{l \tau_{1}} e^{m \tau_{2}} e^{m \tau_{3}}}{\prod_{k=1}^{l}\left(p_{1}+k z\right)^{5} \prod_{k=1}^{m}\left(p_{2}+k z\right)^{5}} \frac{\prod_{k=-\infty}^{0}\left(p_{3}-p_{1}+k z\right)}{\prod_{k=-\infty}^{n-l}\left(p_{3}-p_{1}+k z\right)} \\
\times \frac{\prod_{k=-\infty}^{0}\left(p_{3}-p_{2}+k z\right)}{\prod_{k=-\infty}^{n-m}\left(p_{3}-p_{2}+k z\right)},
\end{array}
$$

where $\tau=\tau_{1} p_{1}+\tau_{2} p_{2}+\tau_{3} p_{3}$ and we have identified the group ring $\mathbb{Q}\left[H_{2}(A / / T ; \mathbb{Z})\right]$ with $\mathbb{Q}\left[Q_{1}, Q_{2}, Q_{3}\right]$ via the $\mathbb{Q}$-linear map that sends $Q^{\beta}$ to $Q_{1}^{\left\langle\beta, p_{1}\right\rangle} Q_{2}^{\left\langle\beta, p_{2}\right\rangle} Q_{3}^{\left\langle\beta, p_{3}\right\rangle}$. The line bundles $L_{1}, L_{2}$ and $H$ are nef, and $c_{1}(A / / T)-c_{1}\left(\mathcal{V}_{T}\right)$ is ample, so Theorem D.3 gives

$$
\begin{aligned}
J_{\boldsymbol{e}, \mathcal{V}_{T}}(\tau)=e^{-Q_{3} e^{\tau} 3 / z} e^{\tau / z} \sum_{l, m, n \geq 0} Q_{1}^{l} & Q_{2}^{m} Q_{3}^{n} e^{l \tau_{1}} e^{m \tau_{2}} e^{m \tau_{3}} \\
& \times \frac{\prod_{k=1}^{n}\left(\lambda+p_{3}+k z\right) \prod_{k=1}^{l+m}\left(\lambda+p_{1}+p_{2}+k z\right)^{3}}{\prod_{k=1}^{l}\left(p_{1}+k z\right)^{5} \prod_{k=1}^{m}\left(p_{2}+k z\right)^{5}} \\
& \times \frac{\prod_{k=-\infty}^{0}\left(p_{3}-p_{1}+k z\right)}{\prod_{k=-\infty}^{n-l}\left(p_{3}-p_{1}+k z\right)} \frac{\prod_{k=-\infty}^{0}\left(p_{3}-p_{2}+k z\right)}{\prod_{k=-\infty}^{n-m}\left(p_{3}-p_{2}+k z\right)} .
\end{aligned}
$$

Consider now $F=A / / G=\mathrm{Fl}$ and a point $t \in H^{\bullet}(F)$. Recall that $\mathrm{Fl}=\mathbb{P}(S)$ is the projectivization of the universal bundle $S$ of subspaces on Gr. Let $\epsilon_{1} \in H^{2}(F ; \mathbb{Q})$ be the pullback to $F$ (under the projection map $q: \mathrm{Fl} \rightarrow \mathrm{Gr}$ ) of the ample generator of $H^{2}(\mathrm{Gr}$ ) and let $\epsilon_{2} \in H^{2}(F ; \mathbb{Q})$ be the first Chern class of $\mathcal{O}_{\mathbb{P}(S)}(1)$. Identify the group ring $\mathbb{Q}\left[H_{2}(F ; \mathbb{Z})\right]$ with $\mathbb{Q}\left[q_{1}, q_{2}\right]$ via the $\mathbb{Q}$-linear map which sends $Q^{\beta}$ to $q_{1}^{\left\langle\beta, \epsilon_{1}\right\rangle} q_{2}^{\left\langle\beta, \epsilon_{2}\right\rangle}$. In [9, Section 6.1] the authors consider the lift $\tilde{J}_{\mathcal{V}_{G}}^{S \times \mathbb{C}^{\times}}(t)$ of their twisted $J$-function $J_{\mathcal{V}_{G}}^{S \times \mathbb{C}^{\times}}(t)$ determined by a choice of lift $H^{\bullet}(A / / G ; \mathbb{Q}) \rightarrow H^{\bullet}(A / / T, \mathbb{Q})^{W}$. We restrict to the locus $t \in H^{0}(A / / G ; \mathbb{Q}) \oplus H^{2}(A / / G ; \mathbb{Q})$, considering the lift

$$
\tilde{J}_{\boldsymbol{e}, \mathcal{V}_{G}}(t):=\tilde{J}_{\mathcal{V}_{G}}^{S \times \mathbb{C}^{\times}}(t), \quad t \in H^{0}(A / / G ; \mathbb{Q}) \oplus H^{2}(A / / G ; \mathbb{Q})
$$

of our twisted $J$-function $J_{\boldsymbol{e}, \mathcal{V}_{G}}$ determined by our choice of lift

$$
H^{\bullet}(A / / G ; \mathbb{Q}) \rightarrow H^{\bullet}(A / / T, \mathbb{Q})^{W}
$$

Theorems 4.1.1 and 6.1.2 in [9] imply that

$$
\tilde{J}_{\boldsymbol{e}, \mathcal{V}_{G}}(\theta(t)) \cup\left(p_{2}-p_{1}\right)=\left[\left(z \frac{\partial}{\partial \tau_{2}}-z \frac{\partial}{\partial \tau_{1}}\right) J_{\boldsymbol{e}, \mathcal{V}_{T}}(\tau)\right]_{\tau=t, Q_{1}=Q_{2}=-q_{1}, Q_{3}=q_{2}}
$$


for some ${ }^{13}$ function $\theta: H^{2}(A / / G ; \mathbb{Q}) \rightarrow H^{\bullet}\left(A / / G ; \Lambda_{G}\right)$. Setting $t=0$ gives $\tilde{J}_{\boldsymbol{e}, \mathcal{V}_{G}}(\theta(0)) \cup\left(p_{2}-p_{1}\right)$

$$
\begin{gathered}
=e^{-q_{2} / z} \sum_{l, m, n \geq 0}(-1)^{l+m} q_{1}^{l+m} q_{2}^{n} \frac{\prod_{k=1}^{n}\left(\lambda+p_{3}+k z\right) \prod_{k=1}^{l+m}\left(\lambda+p_{1}+p_{2}+k z\right)^{3}}{\prod_{k=1}^{l}\left(p_{1}+k z\right)^{5} \prod_{k=1}^{m}\left(p_{2}+k z\right)^{5}} \\
\times \frac{\prod_{k=-\infty}^{0}\left(p_{3}-p_{1}+k z\right)}{\prod_{k=-\infty}^{n-l}\left(p_{3}-p_{1}+k z\right)} \frac{\prod_{k=-\infty}^{0}\left(p_{3}-p_{2}+k z\right)}{\prod_{k=-\infty}^{n-m}\left(p_{3}-p_{2}+k z\right)} \\
\times\left(p_{2}-p_{1}+(m-l) z\right) .
\end{gathered}
$$

For symmetry reasons the right-hand side here is divisible by $p_{2}-p_{1}$; it takes the form

$$
\left(p_{2}-p_{1}\right)\left(1+q_{1} z^{-1}+O\left(z^{-2}\right)\right),
$$

whereas

$$
\tilde{J}_{\boldsymbol{e}, \mathcal{V}_{G}}(\theta(0)) \cup\left(p_{2}-p_{1}\right)=\left(p_{2}-p_{1}\right)\left(1+\theta(0) z^{-1}+O\left(z^{-2}\right)\right) .
$$

We conclude that $\theta(0)=q_{1}$ and hence, via the string equation, that

$$
J_{\boldsymbol{e}, \mathcal{V}_{G}}(\theta(0))=e^{q_{1} / z} J_{\boldsymbol{e}, \mathcal{V}_{G}}(0) .
$$

Thus

$$
\begin{aligned}
& \tilde{J}_{\boldsymbol{e}, \mathcal{V}_{G}}(0) \cup\left(p_{2}-p_{1}\right) \\
& =e^{-\left(q_{1}+q_{2}\right) / z} \sum_{l, m, n \geq 0}(-1)^{l+m} q_{1}^{l+m} q_{2}^{n} \\
& \times \frac{\prod_{k=1}^{n}\left(\lambda+p_{3}+k z\right) \prod_{k=1}^{l+m}\left(\lambda+p_{1}+p_{2}+k z\right)^{3}}{\prod_{k=1}^{l}\left(p_{1}+k z\right)^{5} \prod_{k=1}^{m}\left(p_{2}+k z\right)^{5}} \\
& \times \frac{\prod_{k=-\infty}^{0}\left(p_{3}-p_{1}+k z\right)}{\prod_{k=-\infty}^{n-l}\left(p_{3}-p_{1}+k z\right)} \frac{\prod_{k=-\infty}^{0}\left(p_{3}-p_{2}+k z\right)}{\prod_{k=-\infty}^{n-m}\left(p_{3}-p_{2}+k z\right)} \\
& \times\left(p_{2}-p_{1}+(m-l) z\right) \text {. }
\end{aligned}
$$

We saw in Example D.8 how to extract the quantum period $G_{X}$ from the twisted $J$-function $J_{e}, \mathcal{V}_{G}(0)$ : we take the non-equivariant limit, extract the component along the unit class $1 \in H^{\bullet}(A / / G ; \mathbb{Q})$ and set $z=1$ and $Q^{\beta}=t^{\left\langle\beta,-K_{X}\right\rangle}$. Thus, we consider the right-hand side of (21), take the non-equivariant limit, extract the coefficient of

${ }^{13}$ In fact the mirror map $\theta$ takes values in $H^{0}\left(A / / G ; \Lambda_{G}\right) \oplus H^{2}\left(A / / G ; \Lambda_{G}\right)$. This follows from homogeneity considerations, as in the proof of Proposition D.2. We will see explicitly that $\theta(0) \in H^{0} \oplus H^{2}$. 
$p_{2}-p_{1}$ and set $z=1$ and $q_{1}=q_{2}=t$, obtaining

$$
\begin{aligned}
G_{X}(t)=e^{-2 t} \sum_{l=0}^{\infty} \sum_{m=0}^{\infty} \sum_{n=\max (l, m)}^{\infty}(-1)^{l+m} \frac{n !((l+m) !)^{3}}{(l !)^{5}(m !)^{5}(n-l) !(n-m) !} t^{l+m+n} \\
+e^{-2 t} \sum_{l=0}^{\infty} \sum_{m=l+1}^{\infty} \sum_{n=m}^{\infty}(-1)^{l+m} \frac{n !((l+m) !)^{3}(m-l)}{(l !)^{5}(m !)^{5}(n-l) !(n-m) !} \\
\quad \times\left(5 H_{l}-5 H_{m}+H_{n-m}-H_{n-l}\right) t^{l+m+n} \\
+e^{-2 t} \sum_{l=0}^{\infty} \sum_{m=0}^{\infty} \sum_{n=l}^{m-1}(-1)^{l+n} \frac{n !((l+m) !)^{3}(m-l)(m-n-1) !}{(l !)^{5}(m !)^{5}(n-l) !} t^{l+m+n} .
\end{aligned}
$$

Regularizing yields

$$
\begin{aligned}
\widehat{G}_{X}(t)=1+6 t^{2}+24 t^{3}+138 t^{4}+1080 t^{5}+6540 t^{6} \\
+50400 t^{7}+362250 t^{8}+2713200 t^{9}+\cdots .
\end{aligned}
$$

Minkowski period sequence 69

\section{The Fano manifold $\mathrm{MM}_{2-23}$}

Mori-Mukai construction The blow-up of a quadric 3-fold $Q \subset \mathbb{P}^{4}$ with centre an intersection of $A \in\left|\mathcal{O}_{Q}(1)\right|$ and $B \in\left|\mathcal{O}_{Q}(2)\right|$ such that either

(a) $A$ is nonsingular, or

(b) $A$ is singular.

Our construction A codimension- 2 complete intersection $X$ of type $(L+M) \cap(2 L)$ in the toric variety $F$ with weight data

\begin{tabular}{cccccccc}
$s_{0}$ & $s_{1}$ & $s_{2}$ & $s_{3}$ & $s_{4}$ & $x$ & $x_{5}$ & \\
\cline { 1 - 6 } 1 & 1 & 1 & 1 & 1 & -1 & 0 & $L$ \\
0 & 0 & 0 & 0 & 0 & 1 & 1 & $M$
\end{tabular}

and Nef $F=\langle L, M\rangle$. We have:

- $-K_{F}=4 L+2 M$ is ample, that is $F$ is a Fano variety.

- $X$ is the intersection of two nef divisors on $F$.

- $-\left(K_{F}+\Lambda\right) \sim L+M$ is ample. 
The two constructions coincide Apply Lemma E.1 with $V=\mathcal{O}_{Q}(-1) \oplus \mathcal{O}_{Q}, W=$ $\mathcal{O}_{Q}(1)$ and $f: V \rightarrow W$ given by the matrix $(B A)$. This exhibits $X$ as a member of $\left|\pi^{\star} W(1)\right|$ on $\mathbb{P}(V)$ or, in other words, as a complete intersection of type $(L+M) \cap(2 L)$ on the toric variety $F$.

The quantum period Corollary D.5 yields

$$
G_{X}(t)=e^{-t} \sum_{l=0}^{\infty} \sum_{m=l}^{\infty} t^{l+m} \frac{(l+m) !(2 l) !}{(l !)^{5}(m-l) ! m !}
$$

and regularizing gives

$$
\begin{aligned}
\widehat{G}_{X}(t)=1+8 t^{2}+12 t^{3}+216 t^{4}+720 t^{5} & +8540 t^{6} \\
& +42000 t^{7}+410200 t^{8}+2503200 t^{9}+\cdots .
\end{aligned}
$$

Minkowski period sequence 78

\section{The Fano manifold $\mathrm{MM}_{2-24}$}

Mori-Mukai construction A divisor of bidegree $(1,2)$ on $\mathbb{P}^{2} \times \mathbb{P}^{2}$.

Our construction A member $X$ of $|L+2 M|$ in the toric variety $F=\mathbb{P}^{2} \times \mathbb{P}^{2}$.

The two constructions coincide Obvious.

The quantum period The toric variety $F$ has weight data

$$
\begin{aligned}
& \begin{array}{llllllll}
1 & 1 & 1 & 0 & 0 & 0 & L
\end{array} \\
& \begin{array}{llllllll}
0 & 0 & 0 & 1 & 1 & 1 & M
\end{array}
\end{aligned}
$$

and Nef $F=\langle L, M\rangle$. We have:

- $F$ is a Fano variety.

- $\quad X \sim L+2 M$ is ample.

- $-\left(K_{F}+X\right) \sim 2 L+M$ is ample.

Corollary D.5 yields

$$
G_{X}(t)=e^{-2 t} \sum_{l=0}^{\infty} \sum_{m=0}^{\infty} t^{2 l+m} \frac{(l+2 m) !}{(l !)^{3}(m !)^{3}}
$$


and regularizing gives

$$
\begin{aligned}
\widehat{G}_{X}(t)=1+4 t^{2}+24 t^{3}+132 t^{4}+780 t^{5} & +5800 t^{6} \\
& +40320 t^{7}+283780 t^{8}+2105880 t^{9}+\cdots .
\end{aligned}
$$

Minkowski period sequence 44

\section{The Fano manifold $\mathrm{MM}_{2-25}$}

Mori-Mukai construction The blow up of $\mathbb{P}^{3}$ with centre an elliptic curve that is an intersection of two quadrics.

Our construction A member $X$ of $|L+2 M|$ in the toric variety $F=\mathbb{P}^{1} \times \mathbb{P}^{3}$.

The two constructions coincide Apply Lemma E.1 with $V=\mathcal{O}_{\mathbb{P}^{3}} \oplus \mathcal{O}_{\mathbb{P}^{3}}, W=$ $\mathcal{O}_{\mathbb{P}^{3}}(2)$ and $f: V \rightarrow W$ the map given by the two quadrics that define the elliptic curve.

The quantum period The toric variety $F$ has weight data

$$
\begin{aligned}
& \begin{array}{llllllll}
1 & 1 & 0 & 0 & 0 & 0 & L
\end{array} \\
& \begin{array}{llllllll}
0 & 0 & 1 & 1 & 1 & 1 & M
\end{array}
\end{aligned}
$$

and Nef $F=\langle L, M\rangle$. We have:

- $F$ is a Fano variety.

- $X \sim L+2 M$ is ample.

- $-\left(K_{F}+X\right) \sim L+2 M$ is ample.

Corollary D.5 yields

and regularizing gives

$$
G_{X}(t)=e^{-t} \sum_{l=0}^{\infty} \sum_{m=0}^{\infty} t^{l+2 m} \frac{(l+2 m) !}{(l !)^{2}(m !)^{4}}
$$

$$
\begin{aligned}
\widehat{G}_{X}(t)=1+4 t^{2}+24 t^{3}+60 t^{4}+720 t^{5} & +3640 t^{6} \\
& +21840 t^{7}+175420 t^{8}+1024800 t^{9}+\cdots .
\end{aligned}
$$

Minkowski period sequence 43 


\section{The Fano manifold $M_{2-26}$}

Mori-Mukai construction The blow-up of $B_{5} \subset \mathbb{P}^{6}$ with centre a line on it.

Our construction Let $S$ be the universal bundle of subspaces over $\operatorname{Gr}=\operatorname{Gr}(2,4)$ and let $E$ be the rank-3 vector bundle $E=\mathbb{C} \oplus S^{\star}$ on Gr. Let $q: \mathbb{P}(E) \rightarrow$ Gr denote the projection. Then $X$ is the vanishing locus of a general section of

$$
q^{\star} \operatorname{det} S^{\star} \oplus\left(\left(q^{\star} \operatorname{det} S^{\star}\right) \otimes \mathcal{O}_{\mathbb{P}(E)}(1)\right)^{\oplus 2}
$$

on the key variety $F=\mathbb{P}(E)$.

The two constructions coincide Write $V=\mathbb{C}^{5}$ with basis $e_{0}, \ldots, e_{4}$ and write $\mathbb{C}^{4}=V / \mathbb{C} e_{0}$. Consider Gr as the Grassmannian of 2-dimensional subspaces of this $\mathbb{C}^{4}$. There is an exact sequence

$$
0 \rightarrow T \rightarrow q^{\star} E^{\star} \rightarrow \mathcal{O}_{\mathbb{P}(E)}(1) \rightarrow 0
$$

on $F=\mathbb{P}(E)$, where $T$ is a rank-2 vector bundle.

First we construct a morphism $p: F \rightarrow \operatorname{Gr}(2, V)=\operatorname{Gr}\left(2, \mathbb{C}^{5}\right)$. Let $U$ denote the universal bundle of subspaces on $\operatorname{Gr}(2,5)$. The morphism $p$ arises, by the universal property of $\operatorname{Gr}\left(2, \mathbb{C}^{5}\right)$, from the inclusion

$$
T \subset q^{\star} E^{\star}=\mathbb{C} \oplus q^{\star} S \subset \mathbb{C} \oplus q^{\star} \mathbb{C}^{4}=\mathbb{C} e_{0} \oplus \mathbb{C}^{4}=\mathbb{C}^{5},
$$

ie there is a unique $p: F \rightarrow \operatorname{Gr}\left(2, \mathbb{C}^{5}\right)$ such that $S=p^{\star} U$.

Next we claim that the morphism $p: F \rightarrow \operatorname{Gr}(2,5)$ that we just constructed is the blow-up of $\operatorname{Gr}(2,5)$ along the locus

$$
Z=\left\{W_{2} \subset \mathbb{C}^{5} \mid e_{0} \in W_{2}\right\}
$$

of 2-dimensional vector subspaces that contain $e_{0}$. Denote by $\pi: \mathbb{C}^{5} \rightarrow \mathbb{C}^{5} / \mathbb{C} e_{0}$ the natural projection. Indeed, for $W_{2} \in \operatorname{Gr}(2,5)$, either

- $e_{0} \notin W_{2}$, in which case $\pi\left(W_{2}\right)=V_{2} \subset \mathbb{C}^{4}$ is a 2-dimensional subspace and $p$ is an isomorphism above $W_{2}$, or

- $e_{0} \in W_{2}$, in which case $\pi\left(W_{2}\right)$ is a 1 -dimensional subspace and

$$
q\left(p^{-1} W_{2}\right)=\left\{V_{2} \in \operatorname{Gr}(2,4) \mid \pi\left(W_{2}\right) \subset V_{2}\right\} .
$$

The statement follows easily from the claim just shown. Indeed, on the one hand $Z \cong \mathbb{P}^{3}$ and the Plücker embedding of $\operatorname{Gr}(2,5)$ embeds $Z$ linearly in $\mathbb{P}^{9}$. In other 
words, $p: F \rightarrow \operatorname{Gr}(2,5)$ is the blow up of $\operatorname{Gr}(2,5) \subset \mathbb{P}^{9}$ along a $\mathbb{P}^{3} \subset \operatorname{Gr}(2,5)$. On the other hand, the rational map

$$
q p^{-1}: \operatorname{Gr}(2,5) \rightarrow \operatorname{Gr}(2,4) \subset \mathbb{P}^{5},
$$

where $\operatorname{Gr}(2,4) \subset \mathbb{P}^{5}$ is the Plücker embedding of $\operatorname{Gr}(2,4)$, is the map corresponding to the linear system of hyperplane sections of $\operatorname{Gr}(2,5) \subset \mathbb{P}^{9}$ — in its Plücker embedding that contain $Z$.

In other words, let $Y \subset \operatorname{Gr}(2,4)$ be a general hyperplane section and $H_{1}, H_{2} \subset \operatorname{Gr}(2,5)$ be two general hyperplane sections of $\operatorname{Gr}(2,5)$; then

$$
p: q^{-1}(Y) \cap p^{-1}\left(H_{1} \cap H_{2}\right) \rightarrow p q^{-1}(Y) \cap H_{1} \cap H_{2}
$$

is the blow-up of $B_{5}=p q^{-1}(Y) \cap H_{1} \cap H_{2} \subset \operatorname{Gr}(2,5)$ along the line $Z \cap B_{5}$.

Abelianization Consider the situation as in [9, Section 3.1] with

- the space that is denoted by $X$ in [9] set equal to $A=\mathbb{C}^{11}$, regarded as the space of pairs

$\left\{(v, w) \mid v\right.$ is a $2 \times 4$ complex matrix and $w \in \mathbb{C}^{3}$ is a column vector $\}$;

- $G=\mathrm{GL}_{2}(\mathbb{C}) \times \mathbb{C}^{\star}$, acting on $A$ as

$$
(g, \lambda):(v, w) \mapsto(g v, \lambda \rho(g) w),
$$

where $\mathrm{GL}_{2}(\mathbb{C})$ acts by left multiplication on $M(2,4)$ and $\rho=\rho_{\text {std }} \oplus 0$ is the direct sum of a copy of the standard representation of $\mathrm{GL}_{2}(\mathbb{C})$ and a copy of the trivial representation;

- $T=\left(\mathbb{C}^{\times}\right)^{3}$, the diagonal subtorus in $G$;

- the group that is denoted by $S$ in [9] set equal to the trivial group;

- $\mathcal{V}$ equal to the representation of $G$ given by

$$
\psi \oplus\left(\chi_{3} \otimes \psi\right)^{\oplus 2},
$$

where $\psi: G \rightarrow \mathbb{C}^{\times}$is det $\rho_{\text {std }}$ on the first factor and trivial on the second factor, and $\chi_{3}: G \rightarrow \mathbb{C}^{\times}$is trivial on the first factor and the identity on the second factor.

Then, by Lemma E.2, $A / / G$ is the key variety $F=\mathbb{P}(E)$ introduced above, whereas $A / / T$ is the toric variety with weight data

$$
\begin{array}{llllllllllll}
1 & 1 & 1 & 1 & 0 & 0 & 0 & 0 & 1 & 0 & 0 & L_{1} \\
0 & 0 & 0 & 0 & 1 & 1 & 1 & 1 & 0 & 1 & 0 & L_{2} \\
0 & 0 & 0 & 0 & 0 & 0 & 0 & 0 & 1 & 1 & 1 & L_{3}
\end{array}
$$


and Nef $=\left\langle L_{1}, L_{2}, L_{1}+L_{2}+L_{3}\right\rangle$, that is, $A / / T$ is the projective bundle

$$
\mathbb{P}(\mathcal{O}(-1,0) \oplus \mathcal{O}(0,-1) \oplus \mathcal{O}(-1,-1))
$$

over $\mathbb{P}^{3} \times \mathbb{P}^{3}$. The Weyl group $W=\mathbb{Z} / 2 \mathbb{Z}$ exchanges the first and second factors of $\mathbb{P}^{3} \times \mathbb{P}^{3}$, that is, it exchanges the first set of four co-ordinates with the second set of four co-ordinates in the table giving the weight data. The representation $\mathcal{V}$ induces the vector bundle $q^{\star} \operatorname{det} S^{\star} \oplus\left(\left(q^{\star} \operatorname{det} S^{\star}\right)(1)\right)^{\oplus 2}$ over $A / / G=F$, whereas the representation $\mathcal{V}$ induces the vector bundle

$$
\left(L_{1}+L_{2}\right) \oplus\left(L_{1}+L_{2}+L_{3}\right)^{\oplus 2}
$$

on $A / / T$.

The abelian/non-abelian correspondence Let $p_{1}, p_{2}, p_{3} \in H^{2}(A / / T ; \mathbb{Q})$ denote the first Chern classes of the line bundles $L_{1}, L_{2}$ and $L_{1} \otimes L_{2} \otimes L_{3}$, respectively. We fix a lift of $H^{\bullet}(A / / G ; \mathbb{Q})$ to $H^{\bullet}(A / / T, \mathbb{Q})^{W}$ in the sense of [9, Section 3]; as before there are many possible choices for such a lift and the precise choice made will be unimportant in what follows. The lift allows us to regard $H^{\bullet}(A / / G ; \mathbb{Q})$ as a subspace of $H^{\bullet}(A / / T, \mathbb{Q})^{W}$, which maps isomorphically to the Weyl-anti-invariant part $H^{\bullet}(A / / T, \mathbb{Q})^{a}$ of $H^{\bullet}(A / / T, \mathbb{Q})$ via

$$
H^{\bullet}(A / / T, \mathbb{Q})^{W} \stackrel{\cup\left(p_{2}-p_{1}\right)}{\longrightarrow} H^{\bullet}(A / / T, \mathbb{Q})^{a} .
$$

We compute the quantum period of $X$ by computing the $J$-function of $\mathrm{Fl}=A / / G$ twisted [16] by the Euler class and the bundle $\mathcal{V}_{G}$, using the abelian/non-abelian correspondence [9].

We begin by computing the $J$-function of $A / / T$ twisted by the Euler class and the bundle $\mathcal{V}_{T}$. Consider the bundles $\mathcal{V}_{T}$ and $\mathcal{V}_{G}$ equipped with the canonical $\mathbb{C}^{\times}$-action that rotates fibres and acts trivially on the base. We will compute the twisted $J$-function $J_{\boldsymbol{e}, \mathcal{V}_{T}}$ of $A / / T$ using the quantum Lefschetz theorem; $J_{\boldsymbol{e}, \mathcal{V}_{T}}$ was defined in (6) above and is the restriction to the locus $\tau \in H^{0}(A / / T) \oplus H^{2}(A / / T)$ of what was denoted by $J_{\mathcal{V}_{T}}^{S \times \mathbb{C}^{\times}}(\tau)$ in [9]. The toric variety $A / / T$ is Fano, so Theorem C.1 gives

$$
\begin{array}{r}
J_{A / / T}(\tau)=e^{\tau / z} \sum_{l, m, n \geq 0} \frac{Q_{1}^{l} Q_{2}^{m} Q_{3}^{n} e^{l \tau_{1}} e^{m \tau_{2}} e^{m \tau_{3}}}{\prod_{k=1}^{l}\left(p_{1}+k z\right)^{4} \prod_{k=1}^{m}\left(p_{2}+k z\right)^{4}} \frac{\prod_{k=-\infty}^{0}\left(p_{3}-p_{2}+k z\right)}{\prod_{k=-\infty}^{n-m}\left(p_{3}-p_{2}+k z\right)} \\
\times \frac{\prod_{k=-\infty}^{0}\left(p_{3}-p_{1}+k z\right)}{\prod_{k=-\infty}^{n-l}\left(p_{3}-p_{1}+k z\right)} \frac{\prod_{k=-\infty}^{0}\left(p_{3}-p_{1}-p_{2}+k z\right)}{\prod_{k=-\infty}^{n-l-m}\left(p_{3}-p_{1}-p_{2}+k z\right)},
\end{array}
$$

where $\tau=\tau_{1} p_{1}+\tau_{2} p_{2}+\tau_{3} p_{3}$ and we have identified the group ring $\mathbb{Q}\left[H_{2}(A / / T ; \mathbb{Z})\right]$ with $\mathbb{Q}\left[Q_{1}, Q_{2}, Q_{3}\right]$ via the $\mathbb{Q}$-linear map that sends $Q^{\beta}$ to $Q_{1}^{\left\langle\beta, p_{1}\right\rangle} Q_{2}^{\left\langle\beta, p_{2}\right\rangle} Q_{3}^{\left\langle\beta, p_{3}\right\rangle}$. 
The line bundles $L_{1}+L_{2}$ and $L_{1} \otimes L_{2} \otimes L_{3}$ are nef, and $c_{1}(A / / T)-c_{1}\left(\mathcal{V}_{T}\right)$ is ample, so Theorem D.3 gives

$$
\begin{aligned}
J_{\boldsymbol{e}, \mathcal{V}_{T}}(\tau)=e^{-Q_{3} e^{\tau} 3 / z} e^{\tau / z} \sum_{l, m, n \geq 0} Q_{1}^{l} & Q_{2}^{m} Q_{3}^{n} e^{l \tau_{1}} e^{m \tau_{2}} e^{m \tau_{3}} \\
& \times \frac{\prod_{k=1}^{l+m}\left(\lambda+p_{1}+p_{2}+k z\right) \prod_{k=1}^{n}\left(\lambda+p_{3}+k z\right)^{2}}{\prod_{k=1}^{l}\left(p_{1}+k z\right)^{4} \prod_{k=1}^{m}\left(p_{2}+k z\right)^{4}} \\
& \times \frac{\prod_{k=-\infty}^{0}\left(p_{3}-p_{2}+k z\right)}{\prod_{k=-\infty}^{n-m}\left(p_{3}-p_{1}+k z\right)} \\
& \times \frac{\prod_{k=-\infty}^{0}\left(p_{3}-p_{2}+k z\right)}{\prod_{k=-\infty}^{n-l}\left(p_{3}-p_{1}+k z\right)} \\
\prod_{k=-\infty}^{n-l-m}\left(p_{3}-p_{1}-p_{2}+k z\right) &
\end{aligned}
$$

Consider now $F=A / / G=\mathbb{P}(E)$ and a point $t \in H^{\bullet}(F)$. Let $\epsilon_{1} \in H^{2}(F ; \mathbb{Q})$ be the pullback to $F$ (under the projection map $q: \mathbb{P}(E) \rightarrow \operatorname{Gr}(2,4)$ ) of the ample generator of $H^{2}(\operatorname{Gr}(2,4))$, and let $\epsilon_{2} \in H^{2}(F ; \mathbb{Q})$ be the first Chern class of $\left(q^{\star} \operatorname{det} S^{\star}\right) \otimes \mathcal{O}_{\mathbb{P}(E)}(1)$. Identify the group ring $\mathbb{Q}\left[H_{2}(F ; \mathbb{Z})\right]$ with $\mathbb{Q}\left[q_{1}, q_{2}\right]$ via the $\mathbb{Q}$-linear map which sends $Q^{\beta}$ to $q_{1}^{\left\langle\beta, \epsilon_{1}\right\rangle} q_{2}^{\left\langle\beta, \epsilon_{2}\right\rangle}$. In $[9$, Section 6.1] the authors consider the lift $\tilde{J}_{\mathcal{V}_{G}}^{S \times \mathbb{C}^{\times}}(t)$ of their twisted $J$-function $J_{\mathcal{V}_{G}}^{S \times \mathbb{C}^{\times}}(t)$ determined by a choice of lift $H^{\bullet}(A / / G ; \mathbb{Q}) \rightarrow H^{\bullet}(A / / T, \mathbb{Q})^{W}$. We restrict to the locus $t \in H^{0}(A / / G ; \mathbb{Q}) \oplus H^{2}(A / / G ; \mathbb{Q})$, considering the lift $\tilde{J}_{\boldsymbol{e}, \mathcal{V}_{G}}(t):=\tilde{J}_{\mathcal{V}_{G} \times \mathbb{C}^{\times}}(t)$, $t \in H^{0}(A / / G ; \mathbb{Q}) \oplus H^{2}(A / / G ; \mathbb{Q})$, of our twisted $J$-function $J_{\boldsymbol{e}}, \mathcal{V}_{G}$ determined by our choice of lift $H^{\bullet}(A / / G ; \mathbb{Q}) \rightarrow H^{\bullet}(A / / T, \mathbb{Q})^{W}$. Theorems 4.1.1 and 6.1.2 in [9] imply that

$$
\tilde{J}_{\boldsymbol{e}, \mathcal{V}_{G}}(\theta(t)) \cup\left(p_{2}-p_{1}\right)=\left[\left(z \frac{\partial}{\partial \tau_{2}}-z \frac{\partial}{\partial \tau_{1}}\right) J_{\boldsymbol{e}, \mathcal{V}_{T}}(\tau)\right]_{\tau=t, Q_{1}=Q_{2}=-q_{1}, Q_{3}=q_{2}}
$$

for some ${ }^{14}$ function $\theta: H^{2}(A / / G ; \mathbb{Q}) \rightarrow H^{\bullet}\left(A / / G ; \Lambda_{A / / G}\right)$. Setting $t=0$ gives $\tilde{J}_{\boldsymbol{e}, \mathcal{V}_{G}}(\theta(0)) \cup\left(p_{2}-p_{1}\right)$

$$
\begin{gathered}
e^{-q_{2} / z} \sum_{l, m, n \geq 0}(-1)^{l+m} q_{1}^{l+m} q_{2}^{n} \frac{\prod_{k=1}^{l+m}\left(\lambda+p_{1}+p_{2}+k z\right) \prod_{k=1}^{n}\left(\lambda+p_{3}+k z\right)^{2}}{\prod_{k=1}^{l}\left(p_{1}+k z\right)^{4} \prod_{k=1}^{m}\left(p_{2}+k z\right)^{4}} \\
\times \frac{\prod_{k=-\infty}^{0}\left(p_{3}-p_{2}+k z\right)}{\prod_{k=-\infty}^{n-m}\left(p_{3}-p_{2}+k z\right)} \frac{\prod_{k=-\infty}^{0}\left(p_{3}-p_{1}+k z\right)}{\prod_{k=-\infty}^{n-l}\left(p_{3}-p_{1}+k z\right)} \\
\times \frac{\prod_{k=-\infty}^{0}\left(p_{3}-p_{1}-p_{2}+k z\right)}{\prod_{k=-\infty}^{n-l-m}\left(p_{3}-p_{1}-p_{2}+k z\right)}\left(p_{2}-p_{1}+(m-l) z\right) .
\end{gathered}
$$

${ }^{14}$ As in Theorem F.1 and note 12, the map $\theta$ is grading-preserving and satisfies $\theta \equiv$ id modulo $q_{1}, q_{2}$. 
The left-hand side here takes the form

$$
\left(p_{2}-p_{1}\right)\left(1+\theta(0) z^{-1}+O\left(z^{-2}\right)\right),
$$

whereas the right-hand side is

$$
\left(p_{2}-p_{1}\right)\left(1+O\left(z^{-2}\right)\right)
$$

and therefore $\theta(0)=0$. Thus

$$
\begin{aligned}
& \tilde{J}_{\boldsymbol{e}, \mathcal{V}_{G}}(0) \cup\left(p_{2}-p_{1}\right) \\
& =e^{-q_{2} / z} \sum_{l, m, n \geq 0}(-1)^{l+m} q_{1}^{l+m} q_{2}^{n} \frac{\prod_{k=1}^{l+m}\left(\lambda+p_{1}+p_{2}+k z\right)}{\prod_{k=1}^{l}\left(p_{1}+k z\right)^{4}} \\
& \times \frac{\prod_{k=1}^{n}\left(\lambda+p_{3}+k z\right)^{2}}{\prod_{k=1}^{m}\left(p_{2}+k z\right)^{4}} \frac{\prod_{k=-\infty}^{0}\left(p_{3}-p_{2}+k z\right)}{\prod_{k=-\infty}^{n-m}\left(p_{3}-p_{2}+k z\right)} \\
& \times \frac{\prod_{k=-\infty}^{0}\left(p_{3}-p_{1}+k z\right)}{\prod_{k=-\infty}^{n-l}\left(p_{3}-p_{1}+k z\right)} \frac{\prod_{k=-\infty}^{0}\left(p_{3}-p_{1}-p_{2}+k z\right)}{\prod_{k=-\infty}^{n-l-m}\left(p_{3}-p_{1}-p_{2}+k z\right)} \\
& \times\left(p_{2}-p_{1}+(m-l) z\right) \text {. }
\end{aligned}
$$

We saw in Example D.8 how to extract the quantum period $G_{X}$ from the twisted $J$-function $J_{\boldsymbol{e}, \mathcal{V}_{G}}(0)$ : we take the non-equivariant limit $\lambda \rightarrow 0$, extract the component along the unit class $1 \in H^{\bullet}(A / / G ; \mathbb{Q})$, set $z=1$, and set $Q^{\beta}=t^{\left\langle\beta,-K_{X}\right\rangle}$. Thus we consider the right-hand side of (22), take the non-equivariant limit, extract the coefficient of $p_{2}-p_{1}$ and set $z=1$ and $q_{1}=q_{2}=t$. This yields

$$
\begin{array}{r}
G_{X}(t)=e^{-t} \sum_{l=0}^{\infty} \sum_{m=0}^{\infty} \sum_{n=l+m}^{\infty}(-1)^{l+m} t^{l+m+n} \frac{(l+m) !(n !)^{2}}{(l !)^{4}(m !)^{4}(n-m) !(n-l) !(n-l-m) !} \\
\times\left(1+(m-l)\left(H_{n-m}-4 H_{m}\right)\right),
\end{array}
$$

where $H_{k}$ is the $k^{\text {th }}$ harmonic number. Regularizing gives

$\widehat{G}_{X}(t)=1+6 t^{2}+12 t^{3}+114 t^{4}+540 t^{5}+3480 t^{6}+22680 t^{7}+137970 t^{8}+978600 t^{9}+\cdots$.

Minkowski period sequence 58

\section{The Fano manifold $M_{2-27}$}

Mori-Mukai construction The blow up of $\mathbb{P}^{3}$ with centre a twisted cubic. 
Our construction A codimension-2 complete intersection $X$ of type

$$
(L+M) \cap(L+M)
$$

in the toric variety $F=\mathbb{P}^{3} \times \mathbb{P}^{2}$.

The two constructions coincide The twisted cubic in $\mathbb{P}^{3}$ with co-ordinates $x_{0}, x_{1}$, $x_{2}, x_{3}$ is given by the condition

$$
\operatorname{rk}\left(\begin{array}{lll}
x_{0} & x_{1} & x_{2} \\
x_{1} & x_{2} & x_{3}
\end{array}\right)<2
$$

Applying Lemma E.1 with $V=\mathcal{O}_{\mathbb{P}^{3}}^{\oplus 3}, W=\mathcal{O}_{\mathbb{P}^{3}}(1)^{\oplus 2}$ and the map $f: V \rightarrow W$ given by

$$
\left(\begin{array}{lll}
x_{0} & x_{1} & x_{2} \\
x_{1} & x_{2} & x_{3}
\end{array}\right)
$$

we see that $X$ is cut out of $\mathbb{P}(V)$ by a section of $\pi^{\star} W \otimes \mathcal{O}_{\mathbb{P}(E)}(1)$. In other words, $X$ is a complete intersection in $\mathbb{P}^{3} \times \mathbb{P}^{2}$ of type $(L+M) \cap(L+M)$.

The quantum period The toric variety $F$ has weight data

$$
\begin{array}{llllllll}
1 & 1 & 1 & 1 & 0 & 0 & 0 & \\
0 & 0 & 0 & 0 & 1 & 1 & 1 & M
\end{array}
$$

and Nef $F=\langle L, M\rangle$. We have that:

- $F$ is a Fano variety.

- $X$ is the intersection of two ample divisors on $F$.

- $-\left(K_{F}+\Lambda\right) \sim 2 L+M$ is ample.

Corollary D.5 yields

and regularizing gives

$$
G_{X}(t)=e^{-t} \sum_{l=0}^{\infty} \sum_{m=0}^{\infty} t^{2 l+m} \frac{(l+m) !(l+m) !}{(l !)^{4}(m !)^{3}}
$$

$\widehat{G}_{X}(t)=1+2 t^{2}+18 t^{3}+30 t^{4}+240 t^{5}+1730 t^{6}+5880 t^{7}+41230 t^{8}+262080 t^{9}+\cdots$.

Minkowski period sequence 19

\section{The Fano manifold $\mathrm{MM}_{2-28}$}

Mori-Mukai construction The blow-up of $\mathbb{P}^{3}$ with centre a plane cubic. 
Our construction A member $X$ of $|L+M|$ in the toric variety $F$ with weight data

\begin{tabular}{ccccccc}
$s_{0}$ & $s_{1}$ & $s_{2}$ & $s_{3}$ & $x$ & $y$ & \\
\hline 1 & 1 & 1 & 1 & -2 & 0 & $L$ \\
0 & 0 & 0 & 0 & 1 & 1 & $M$
\end{tabular}

and Nef $F=\langle L, M\rangle$. We have:

- $-K_{F}=2 L+2 M$ is ample, that is, $F$ is a Fano variety.

- $X \sim L+M$ is ample.

- $-\left(K_{F}+X\right) \sim L+M$ is ample.

The two constructions coincide Suppose that the centre of the blow-up is defined by the simultaneous vanishing of $A$ and $B$, where $A$ is a member of $\mathcal{O}_{\mathbb{P}^{3}}(3)$ and $B$ is a member of $\mathcal{O}_{\mathbb{P}^{3}}(1)$. Apply Lemma E.1 with $V=\mathcal{O}_{\mathbb{P}^{3}}(-2) \oplus \mathcal{O}_{\mathbb{P}^{3}}, W=\mathcal{O}_{\mathbb{P}^{3}}(1)$ and the map $f: V \rightarrow W$ given by $(A B)$.

The quantum period Corollary D.5 yields

and regularizing gives

$$
G_{X}(t)=e^{-t} \sum_{l=0}^{\infty} \sum_{m=2 l}^{\infty} t^{l+m} \frac{(l+m) !}{(l !)^{4}(m-2 l) ! m !}
$$

$$
\widehat{G}_{X}(t)=1+18 t^{3}+24 t^{4}+1350 t^{6}+3780 t^{7}+2520 t^{8}+141120 t^{9}+\cdots .
$$

Minkowski period sequence 5

\section{The Fano manifold $M_{2-29}$}

Mori-Mukai construction The blow-up of a quadric 3-fold $Q \subset \mathbb{P}^{3}$ with centre a conic on it.

Our construction A member $X$ of $|2 M|$ in the toric variety $F$ with weight data

\begin{tabular}{ccccccc}
$s_{0}$ & $s_{1}$ & $x$ & $x_{2}$ & $x_{3}$ & $x_{4}$ & \\
\cline { 1 - 5 } 1 & 1 & -1 & 0 & 0 & 0 & $L$ \\
0 & 0 & 1 & 1 & 1 & 1 & $M$
\end{tabular}

and Nef $F=\langle L, M\rangle$. We have:

- $-K_{F}=L+4 M$ is ample, that is, $F$ is a Fano variety.

- $X \sim 2 M$ is nef and big.

- $-\left(K_{F}+X\right) \sim L+2 M$ is ample. 
The two constructions coincide The morphism $F \rightarrow \mathbb{P}^{4}$ that sends (contravariantly) the homogeneous co-ordinate functions $\left[x_{0}, \ldots, x_{4}\right]$ to $\left[x s_{0}, x s_{1}, x_{2}, x_{3}, x_{4}\right]$ blows up the plane $\left(x_{0}=x_{1}=0\right)$ in $\mathbb{P}^{4}$. Thus a generic member of $|2 M|$ on $F$ is the blow-up of a quadric 3 -fold with centre a conic on it.

The quantum period Corollary D.5 yields

and regularizing gives

$$
G_{X}(t)=\sum_{l=0}^{\infty} \sum_{m=l}^{\infty} t^{l+2 m} \frac{(2 m) !}{(l !)^{2}(m-l) !(m !)^{3}}
$$

$\widehat{G}_{X}(t)=1+4 t^{2}+12 t^{3}+36 t^{4}+360 t^{5}+940 t^{6}+8400 t^{7}+38500 t^{8}+210000 t^{9}+\cdots$.

Minkowski period sequence 35

\section{The Fano manifold $\mathrm{MM}_{2-30}$}

Mori-Mukai construction The blow-up of $\mathbb{P}^{3}$ with centre a conic.

Our construction A member $X$ of $|L+M|$ in the toric variety $F$ with weight data

\begin{tabular}{ccccccc}
$s_{0}$ & $s_{1}$ & $s_{2}$ & $s_{3}$ & $x$ & $x_{4}$ & \\
\cline { 1 - 5 } & 1 & 1 & 1 & -1 & 0 & $L$ \\
0 & 0 & 0 & 0 & 1 & 1 & $M$
\end{tabular}

and Nef $F=\langle L, M\rangle$. We have:

- $-K_{F}=3 L+2 M$ is ample, that is, $F$ is a Fano variety.

- $X \sim L+M$ is ample.

- $-\left(K_{F}+X\right) \sim 2 L+M$ is ample.

The two constructions coincide Suppose that the centre of the blow-up is defined by the simultaneous vanishing of $A$ and $B$, where $A$ is a member of $\mathcal{O}_{\mathbb{P}^{3}}(2)$ and $B$ is a member of $\mathcal{O}_{\mathbb{P}^{3}}(1)$. Apply Lemma E.1 with $V=\mathcal{O}_{\mathbb{P}^{3}}(-1) \oplus \mathcal{O}_{\mathbb{P}^{3}}, W=\mathcal{O}_{\mathbb{P}^{3}}(1)$ and the map $f: V \rightarrow W$ given by $(A B)$.

The quantum period Corollary D.5 yields

and regularizing gives

$$
G_{X}(t)=e^{-t} \sum_{l=0}^{\infty} \sum_{m=l}^{\infty} t^{2 l+m} \frac{(l+m) !}{(l !)^{4}(m-l) ! m !}
$$

$$
\widehat{G}_{X}(t)=1+12 t^{3}+24 t^{4}+540 t^{6}+2520 t^{7}+2520 t^{8}+33600 t^{9}+\cdots .
$$




\section{Minkowski period sequence 4}

\section{The Fano manifold $\mathrm{MM}_{2-31}$}

Mori-Mukai construction The blow-up of a quadric 3 -fold $Q \subset \mathbb{P}^{4}$ with centre a line on it.

Our construction A member $X$ of $|L+M|$ in the toric variety $F$ with weight data

\begin{tabular}{ccccccc}
$s_{0}$ & $s_{1}$ & $s_{2}$ & $x$ & $x_{3}$ & $x_{4}$ & \\
\cline { 1 - 5 } 1 & 1 & 1 & -1 & 0 & 0 & $L$ \\
0 & 0 & 0 & 1 & 1 & 1 & $M$
\end{tabular}

and Nef $F=\langle L, M\rangle$. We have:

- $-K_{F}=2 L+3 M$ is ample, that is, $F$ is a Fano variety.

- $X \sim L+M$ is ample.

- $-\left(K_{F}+X\right) \sim L+2 M$ is ample.

The two constructions coincide The morphism $F \rightarrow \mathbb{P}^{4}$ that sends (contravariantly) the homogeneous co-ordinate functions $\left[x_{0}, \ldots, x_{4}\right]$ to $\left[x s_{0}, x s_{1}, x s_{2}, x_{3}, x_{4}\right]$ blows up the line $\left(x_{0}=x_{1}=x_{2}=0\right)$ in $\mathbb{P}^{4}$, and $X$ is the proper transform of a quadric containing this line.

The quantum period Corollary D.5 yields

and regularizing gives

$$
G_{X}(t)=\sum_{l=0}^{\infty} \sum_{m=l}^{\infty} t^{l+2 m} \frac{(l+m) !}{(l !)^{3}(m-l) !(m !)^{2}}
$$

$\widehat{G}_{X}(t)=1+2 t^{2}+12 t^{3}+6 t^{4}+180 t^{5}+560 t^{6}+1680 t^{7}+16870 t^{8}+46200 t^{9}+\cdots$.

Minkowski period sequence 15

\section{The Fano manifold $M_{2-32}$ (also known as $W$ )}

Mori-Mukai construction The divisor $W$ of bidegree $(1,1)$ on $\mathbb{P}^{2} \times \mathbb{P}^{2}$.

Our construction A member $X$ of $|L+M|$ on the toric variety $F=\mathbb{P}^{2} \times \mathbb{P}^{2}$. 
The two constructions coincide Obvious.

The quantum period The toric variety $F$ has weight data

$$
\begin{array}{rrrrrrr}
1 & 1 & 1 & 0 & 0 & 0 & \\
0 & 0 & 0 & 1 & 1 & 1 & M
\end{array}
$$

and Nef $F=\langle L, M\rangle$. We have that:

- $F$ is a Fano variety.

- $X \sim L+M$ is ample.

- $-\left(K_{F}+X\right) \sim 2 L+2 M$ is ample.

Corollary D.5 yields

and regularizing gives

$$
G_{X}(t)=\sum_{l=0}^{\infty} \sum_{m=0}^{\infty} t^{2 l+2 m} \frac{(l+m) !}{(l !)^{3}(m !)^{3}}
$$

$$
\widehat{G}_{X}(t)=1+4 t^{2}+60 t^{4}+1120 t^{6}+24220 t^{8}+567504 t^{10}+\cdots .
$$

Minkowski period sequence 24

\section{The Fano manifold $M_{2-33}$}

Mori-Mukai construction The blow-up of $\mathbb{P}^{3}$ with centre a line.

Our construction The toric Fano variety $X$ with weight data

\begin{tabular}{cccccc}
$s_{0}$ & $s_{1}$ & $x$ & $x_{2}$ & $x_{3}$ & \\
\cline { 1 - 4 } 1 & 1 & -1 & 0 & 0 & $L$ \\
0 & 0 & 1 & 1 & 1 & $M$
\end{tabular}

and $\operatorname{Nef} X=\langle L, M\rangle$.

The two constructions coincide The blow-up $X \rightarrow \mathbb{P}^{3}$ sends (contravariantly) the homogeneous co-ordinate functions $\left[x_{0}, x_{1}, x_{2}, x_{3}\right]$ to $\left[x s_{0}, x s_{1}, x_{2}, x_{3}\right]$.

The quantum period Corollary C.2 yields

and regularizing gives

$$
G_{X}(t)=\sum_{l=0}^{\infty} \sum_{m=l}^{\infty} \frac{t^{l+3 m}}{(l !)^{2}(m-l) !(m !)^{2}}
$$

$$
\widehat{G}_{X}(t)=1+6 t^{3}+24 t^{4}+90 t^{6}+1260 t^{7}+2520 t^{8}+1680 t^{9}+\cdots .
$$


Minkowski period sequence 2

\section{The Fano manifold $\mathrm{MM}_{2-34}$}

Mori-Mukai construction $\mathbb{P}^{1} \times \mathbb{P}^{2}$.

Our construction $\mathbb{P}^{1} \times \mathbb{P}^{2}$.

The quantum period $X=\mathbb{P}^{1} \times \mathbb{P}^{2}$ is the toric Fano variety with weight data

$$
\begin{aligned}
& \begin{array}{lllllll}
1 & 1 & 0 & 0 & 0 & L
\end{array} \\
& \begin{array}{lllllll}
0 & 0 & 1 & 1 & 1 & M
\end{array}
\end{aligned}
$$

and Nef $X=\langle L, M\rangle$. Corollary C.2 yields

$$
G_{X}(t)=\sum_{l=0}^{\infty} \sum_{m=0}^{\infty} \frac{t^{2 l+3 m}}{(l !)^{2}(m !)^{3}}
$$

and regularizing gives

$\widehat{G}_{X}(t)=1+2 t^{2}+6 t^{3}+6 t^{4}+120 t^{5}+110 t^{6}+1260 t^{7}+5110 t^{8}+11760 t^{9}+\cdots$.

Minkowski period sequence 10

\section{The Fano manifold $M_{2-35}$ (also known as $B_{7}$ )}

Mori-Mukai construction $\quad B_{7}$, the blow-up of $\mathbb{P}^{3}$ at a point; equivalently, the $\mathbb{P}^{1}$ bundle $\mathbb{P}(\mathcal{O}+\mathcal{O}(1))$ over $\mathbb{P}^{2}$.

Our construction The toric Fano variety $X$ with weight data

\begin{tabular}{cccccc}
$s_{0}$ & $s_{1}$ & $s_{2}$ & $x$ & $x_{3}$ & \\
\cline { 1 - 4 } & 1 & 1 & -1 & 0 & $L$ \\
0 & 0 & 0 & 1 & 1 & $M$
\end{tabular}

and $\operatorname{Nef} X=\langle L, M\rangle$.

The two constructions coincide The blow-up $X \rightarrow \mathbb{P}^{3}$ sends (contravariantly) the homogeneous co-ordinate functions $\left[x_{0}, x_{1}, x_{2}, x_{3}\right]$ to $\left[x s_{0}, x s_{1}, x s_{2}, x_{3}\right]$. 
The quantum period Corollary C.2 yields

and regularizing gives

$$
G_{X}(t)=\sum_{l=0}^{\infty} \sum_{m=l}^{\infty} \frac{t^{2 l+2 m}}{(l !)^{3}(m-l) ! m !}
$$

$$
\widehat{G}_{X}(t)=1+2 t^{2}+30 t^{4}+380 t^{6}+5950 t^{8}+101052 t^{10}+\cdots .
$$

Minkowski period sequence 7

\section{The Fano manifold $\mathrm{MM}_{2-36}$}

Mori-Mukai construction The blow-up of the Veronese cone $W_{4} \subset \mathbb{P}^{6}$ with centre the vertex; equivalently, the $\mathbb{P}^{1}$-bundle $\mathbb{P}(\mathcal{O} \oplus \mathcal{O}(2))$ over $\mathbb{P}^{2}$.

Our construction The toric Fano variety $X$ with weight data

\begin{tabular}{cccccc}
$s_{0}$ & $s_{1}$ & $s_{2}$ & $x$ & $y$ & \\
\cline { 1 - 4 } 1 & 1 & 1 & -2 & 0 & $L$ \\
0 & 0 & 0 & 1 & 1 & $M$
\end{tabular}

and $\operatorname{Nef} F=\langle L, M\rangle$.

The two constructions coincide Obvious.

The quantum period Corollary C.2 yields

and regularizing gives

$$
G_{X}(t)=\sum_{l=0}^{\infty} \sum_{m=2 l}^{\infty} \frac{t^{l+2 m}}{(l !)^{3}(m-2 l) ! m !}
$$

$$
\widehat{G}_{X}(t)=1+2 t^{2}+6 t^{4}+60 t^{5}+20 t^{6}+840 t^{7}+70 t^{8}+7560 t^{9}+\cdots .
$$

Minkowski period sequence 6

\section{The Fano manifold $\mathrm{MM}_{3-1}$}

Mori-Mukai construction A double cover of $\mathbb{P}^{1} \times \mathbb{P}^{1} \times \mathbb{P}^{1}$ branched along a divisor of tridegree $(2,2,2)$. 
Our construction A member $X$ of $|2 L+2 M+2 N|$ in the toric variety $F$ with weight data

\begin{tabular}{cccccccc}
$x_{0}$ & $x_{1}$ & $y_{0}$ & $y_{1}$ & $z_{0}$ & $z_{1}$ & $w$ & \\
\cline { 1 - 5 } 1 & 1 & 0 & 0 & 0 & 0 & 1 & \\
0 & 0 & 1 & 1 & 0 & 0 & 1 & $M$ \\
0 & 0 & 0 & 0 & 1 & 1 & 1 & $N$
\end{tabular}

and Nef $F=\langle L, M, L+M+N\rangle$. The secondary fan for $F$ has three maximal cones; the corresponding three toric varieties are isomorphic. It is easy to see that Nef $X=\langle L, M, N\rangle$. We have:

- $-K_{F}=3(L+M+N)$ is nef and big but not ample.

- $\quad X \sim 2(L+M+N)$ is nef and big but not ample.

- $-\left(K_{F}+X\right) \sim L+M+N$ is nef and big but not ample.

The two constructions coincide Consider the equation $w^{2}=f\left(x_{0}, x_{1}, y_{0}, y_{1}, z_{0}, z_{1}\right)$, where $f$ is a generic polynomial of degree 2 in $x_{0}$ and $x_{1}$, degree 2 in $y_{0}$ and $y_{1}$, and degree 2 in $z_{0}$ and $z_{1}$.

The quantum period Let $p_{1}, p_{2}, p_{3} \in H^{\bullet}(F ; \mathbb{Z})$ denote the first Chern classes of $L, M$ and $L \otimes M \otimes N$, respectively; these classes form a basis for $H^{2}(F ; \mathbb{Z})$. Write $\tau \in H^{2}(F ; \mathbb{Q})$ as $\tau=\tau_{1} p_{1}+\tau_{2} p_{2}+\tau_{3} p_{3}$ and identify the group ring $\mathbb{Q}\left[H_{2}(F ; \mathbb{Z})\right]$ with the polynomial ring $\mathbb{Q}\left[Q_{1}, Q_{2}, Q_{3}\right]$ via the $\mathbb{Q}$-linear map that sends the element $Q^{\beta} \in \mathbb{Q}\left[H_{2}(F ; \mathbb{Z})\right]$ to $Q_{1}^{\left\langle\beta, p_{1}\right\rangle} Q_{2}^{\left\langle\beta, p_{2}\right\rangle} Q_{3}^{\left\langle\beta, p_{3}\right\rangle}$. We have

$$
\begin{aligned}
I_{F}(\tau)=e^{\tau / z} \sum_{l, m, n \geq 0} \frac{Q_{1}^{l} Q_{2}^{m} Q_{3}^{n} e^{l \tau_{1}} e^{m \tau_{2}} e^{n \tau_{3}}}{\prod_{k=1}^{l}\left(p_{1}+k z\right)^{2} \prod_{k=1}^{m}\left(p_{2}+k z\right)^{2} \prod_{k=1}^{n}\left(p_{3}+k z\right)} & \times \frac{\prod_{k=-\infty}^{0}\left(p_{3}-p_{1}-p_{2}+k z\right)^{2}}{\prod_{k=-\infty}^{n-l-m}\left(p_{3}-p_{1}-p_{2}+k z\right)^{2}} \\
=1+\tau z^{-1}+O\left(z^{-2}\right) . &
\end{aligned}
$$

Theorem C.1 gives

$$
J_{F}(\tau)=I_{F}(\tau)
$$

and hence

$$
\begin{array}{r}
I_{e, E}(\tau)=e^{\tau / z} \sum_{l, m, n \geq 0} \frac{Q_{1}^{l} Q_{2}^{m} Q_{3}^{n} e^{l \tau_{1}} e^{m \tau_{2}} e^{n \tau_{3}} \prod_{k=1}^{2 n}\left(\lambda+2 p_{3}+k z\right)}{\prod_{k=1}^{l}\left(p_{1}+k z\right)^{2} \prod_{k=1}^{m}\left(p_{2}+k z\right)^{2} \prod_{k=1}^{n}\left(p_{3}+k z\right)} \\
\times \frac{\prod_{k=-\infty}^{0}\left(p_{3}-p_{1}-p_{2}+k z\right)^{2}}{\prod_{k=-\infty}^{n-l-m}\left(p_{3}-p_{1}-p_{2}+k z\right)^{2}}
\end{array}
$$


Since

$$
I_{\boldsymbol{e}, E}(\tau)=1+\left(\tau+2 Q_{3}+2 Q_{1} Q_{3}+2 Q_{2} Q_{3}\right) z^{-1}+O\left(z^{-2}\right),
$$

applying Theorem D.3 yields

$$
J_{\boldsymbol{e}, E}\left(\tau+2 Q_{3}+2 Q_{1} Q_{3}+2 Q_{2} Q_{3}\right)=I_{\boldsymbol{e}, E}(\tau) .
$$

The string equation now implies that

$$
J_{\boldsymbol{e}, E}(\tau)=e^{-\left(2 Q_{3}+2 Q_{1} Q_{3}+2 Q_{2} Q_{3}\right) / z} I_{\boldsymbol{e}, E}(\tau)
$$

and taking the non-equivariant limit $\lambda \rightarrow 0$ gives

$J_{F, X}(\tau)=e^{-\left(2 Q_{3}+2 Q_{1} Q_{3}+2 Q_{2} Q_{3}\right) / z} e^{\tau / z}$

$$
\begin{array}{r}
\times \sum_{l, m, n \geq 0} \frac{Q_{1}^{l} Q_{2}^{m} Q_{3}^{n} e^{l \tau_{1}} e^{m \tau_{2}} e^{n \tau_{3}} \prod_{k=1}^{2 n}\left(2 p_{3}+k z\right)}{\prod_{k=1}^{l}\left(p_{1}+k z\right)^{2} \prod_{k=1}^{m}\left(p_{2}+k z\right)^{2} \prod_{k=1}^{n}\left(p_{3}+k z\right)} \\
\times \frac{\prod_{k=-\infty}^{0}\left(p_{3}-p_{1}-p_{2}+k z\right)^{2}}{\prod_{k=-\infty}^{n-l-m}\left(p_{3}-p_{1}-p_{2}+k z\right)^{2}} .
\end{array}
$$

We now proceed exactly as in the proof of Corollary D.5, obtaining

$$
G_{X}(t)=e^{-6 t} \sum_{l=0}^{\infty} \sum_{m=0}^{\infty} \sum_{n=l+m}^{\infty} t^{n} \frac{(2 n) !}{(l !)^{2}(m !)^{2} n !((n-l-m) !)^{2}} .
$$

Regularizing gives

$$
\begin{aligned}
\widehat{G}_{X}(t)=1+54 t^{2}+672 t^{3}+15642 t^{4}+336960 t^{5}+7919460 t^{6} \\
+191177280 t^{7}+4751272890 t^{8}+120527514240 t^{9}+\cdots
\end{aligned}
$$

Minkowski period sequence 154

\section{The Fano manifold $\mathrm{MM}_{3-2}$}

Mori-Mukai construction A member of $\left|\iota^{\otimes 2} \otimes_{\mathcal{O}_{\mathbb{P}^{1} \times \mathbb{P}^{1}}} \mathcal{O}_{\mathbb{P}^{1} \times \mathbb{P}^{1}}(2,3)\right|$ on the $\mathbb{P}^{2}$ bundle

$$
\mathbb{P}\left(\mathcal{O}_{\mathbb{P}^{1} \times \mathbb{P}^{1}} \oplus \mathcal{O}_{\mathbb{P}^{1} \times \mathbb{P}^{1}}(-1,-1)^{\oplus 2}\right)
$$

over $\mathbb{P}^{1} \times \mathbb{P}^{1}$ such that $X \cap Y$ is irreducible, where $€$ is the tautological line bundle (that is, the fibrewise $\mathcal{O}(1)$ on the $\mathbb{P}^{2}$-bundle) and $Y$ is a member of $|\mathrm{E}|$. 


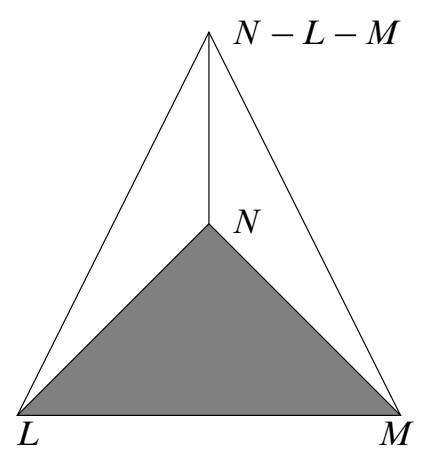

Figure 1: The $M$ secondary fan for $F$ in $\mathrm{MM}_{3-2}$.

Our construction A member $X$ of $|M+2 N|$ in the toric variety $F$ with weight data

\begin{tabular}{cccccccc}
$x_{0}$ & $x_{1}$ & $y_{0}$ & $y_{1}$ & $t$ & $t_{0}$ & $t_{1}$ & \\
\cline { 1 - 5 } 1 & 1 & 0 & 0 & -1 & 0 & 0 & $L$ \\
0 & 0 & 1 & 1 & -1 & 0 & 0 & $M$ \\
0 & 0 & 0 & 0 & 1 & 1 & 1 & $N$
\end{tabular}

and $\operatorname{Nef} F=\langle L, M, N\rangle$.

We have:

- $-K_{F}=L+M+3 N$ is ample, that is, $F$ is a Fano variety.

- $\quad X \sim M+2 N$ is nef and big.

- $-\left(K_{F}+X\right) \sim L+N$ is nef and big but not ample on $F$ (it is ample when restricted to $X$ ).

The two constructions coincide Mori and Mukai use different weight conventions to ours, so their construction exhibits $X$ as a member of $\left|2 L^{\prime}+3 M^{\prime}+2 N^{\prime}\right|$ in the toric variety with weight data

$$
\begin{aligned}
& \begin{array}{lllllllll}
1 & 1 & 0 & 0 & 0 & 1 & 1 & L^{\prime}
\end{array} \\
& \begin{array}{llllllllll}
0 & 0 & 1 & 1 & 0 & 1 & 1 & M^{\prime}
\end{array} \\
& \begin{array}{lllllllll}
0 & 0 & 0 & 0 & 1 & 1 & 1 & N^{\prime}
\end{array}
\end{aligned}
$$

and Nef $F=\left\langle L^{\prime}, M^{\prime}, L^{\prime}+M^{\prime}+N^{\prime}\right\rangle$. Changing basis yields our construction.

Remarks on our construction Note that the secondary fan for $F$ has three maximal cones, as in Figure 1.

The following table gives more detail about the irrelevant ideal, unstable locus and quotient variety corresponding to each of the maximal cones of the secondary fan: 


\begin{tabular}{cccc}
\hline Chamber & Irrelevant ideal & Unstable locus & $\mathbb{C}^{7} / / \omega^{T}$ \\
\hline$\langle L, M, N\rangle$ & $\left(x_{i} y_{j} t_{k}, x_{i} y_{j} t\right)\left(x_{0}=x_{1}=0\right) \cup\left(y_{0}=y_{1}=0\right) \cup\left(t=t_{0}=t_{1}=0\right)$ & $F$ \\
$\langle L, N, N-L-M\rangle$ & $\left(x_{i} t_{k} t, x_{i} y_{j} t\right)(t=0) \cup\left(x_{0}=x_{1}=0\right) \cup\left(y_{0}=y_{1}=t_{0}=t_{1}=0\right)$ & $G$ \\
$\langle M, N, N-L-M\rangle$ & $\left(y_{j} t_{k} t, x_{i} y_{j} t\right)(t=0) \cup\left(y_{0}=y_{1}=0\right) \cup\left(x_{0}=x_{1}=t_{0}=t_{1}=0\right)$ & $G^{\prime}$ \\
\hline
\end{tabular}

The shape of the unstable locus shows that the second and third maximal cones are "hollow", that is, taking the GIT quotient with respect to these stability conditions leads to toric varieties of Picard rank 2 . We discuss briefly the variety $G$, which is the most relevant for understanding the geometry of $X$. Since $t \neq 0$, we can use the $M$-torus to reduce to $t=1$ and eliminate $t$. We are left with the toric variety $G$ with weight data

\begin{tabular}{ccccccc}
$x_{0}$ & $x_{1}$ & $u_{0}$ & $u_{1}$ & $t_{0}$ & $t_{1}$ & \\
\hline 1 & 1 & -1 & -1 & 0 & 0 & $L^{\prime}$ \\
0 & 0 & 1 & 1 & 1 & 1 & $N^{\prime}$
\end{tabular}

and $\operatorname{Nef} G=\left\langle L^{\prime}, N^{\prime}\right\rangle$. The morphism $f: F \rightarrow G$ is given (contravariantly) by

$$
\left[x_{0}, x_{1}, u_{0}, u_{1}, t_{0}, t_{1}\right] \mapsto\left[x_{0}, x_{1}, t y_{0}, t y_{1}, t_{0}, t_{1}\right]
$$

and we have $L=f^{\star} L^{\prime}$ and $N=f^{\star} N^{\prime}$.

The divisor that Mori and Mukai denote by $Y$ is, in our notation,

$$
(t=0) \cong \mathbb{P}_{x_{0}, x_{1}}^{1} \times \mathbb{P}_{y_{0}, y_{1}}^{1} \times \mathbb{P}_{t_{0}, t_{1}}^{1} .
$$

The complete linear system $\left|-\left(K_{F}+X\right)\right|$ defines the morphism $f: F \rightarrow G$, which (a) contracts the divisor $Y$ to $\mathbb{P}_{x_{0}, x_{1}}^{1} \times \mathbb{P}_{t_{0}, t_{1}}^{1}$ and (b) is an isomorphism of $X$ to its image. Under $f: F \rightarrow G, X$ maps isomorphically to a member $X^{\prime}$ of $\left|-L^{\prime}+3 N^{\prime}\right|$ on $G$. This makes it clear that $X$ is Fano, because $-\left(K_{G}+X^{\prime}\right)=L^{\prime}+N^{\prime}$ is ample on $G$; however, because $X^{\prime}$ is not nef on $G$, this construction, economical though it is, is useless for calculating the quantum cohomology of $X$, as the convexity assumption on the bundle in quantum Lefschetz is not satisfied.

The quantum period This is Example D.8. We have

$$
\begin{aligned}
\widehat{G}_{X}(t)=1+58 t^{2}+600 t^{3} & +13182 t^{4}+247440 t^{5}+5212300 t^{6} \\
+ & 111835920 t^{7}+2480747710 t^{8}+56184565920 t^{9}+\cdots
\end{aligned}
$$




\section{The Fano manifold $\mathrm{MM}_{3-3}$}

Mori-Mukai construction A divisor of tridegree $(1,1,2)$ on $\mathbb{P}^{1} \times \mathbb{P}^{1} \times \mathbb{P}^{2}$.

Our construction A member $X$ of $|L+M+2 N|$ on the toric variety $F$ with weight data

$\begin{array}{rrrrrrrr}1 & 1 & 0 & 0 & 0 & 0 & 0 & L \\ 0 & 0 & 1 & 1 & 0 & 0 & 0 & M \\ 0 & 0 & 0 & 0 & 1 & 1 & 1 & N\end{array}$

and Nef $F=\langle L, M, N\rangle$.

The two constructions coincide Obvious.

The quantum period We have:

- $F$ is a Fano variety.

- $X \sim L+M+2 N$ is ample.

- $-\left(K_{F}+X\right) \sim L+M+N$ is ample.

Corollary D.5 yields

$$
G_{X}(t)=e^{-4 t} \sum_{l=0}^{\infty} \sum_{m=0}^{\infty} \sum_{n=0}^{\infty} t^{l+m+n} \frac{(l+m+2 n) !}{(l !)^{2}(m !)^{2}(n !)^{3}}
$$

and regularizing gives

$$
\begin{array}{rl}
\widehat{G}_{X}(t)=1+20 t^{2}+132 t^{3}+18 & 12 t^{4}+21720 t^{5}+289100 t^{6} \\
& +3927840 t^{7}+54999700 t^{8}+785606640 t^{9}+\cdots .
\end{array}
$$

Minkowski period sequence 135

\section{The Fano manifold $\mathrm{MM}_{3-4}$}

Mori-Mukai construction The blow-up of the variety $\mathrm{MM}_{2-18}$ with centre a smooth fibre of the composition

$$
\mathrm{MM}_{2-18} \stackrel{\text { double cover }}{\longrightarrow} \mathbb{P}^{2} \times \mathbb{P}^{1} \stackrel{\text { projection }}{\longrightarrow} \mathbb{P}^{2} .
$$




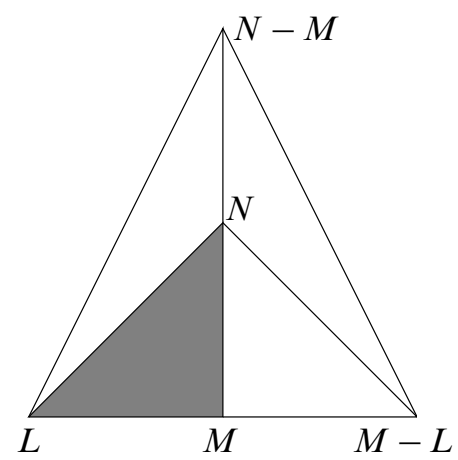

Figure 2: The secondary fan for $F$ in $\mathrm{MM}_{3-4}$.

Our construction A member $X$ of $|2 N|$ on the toric variety $F$ with weight data

\begin{tabular}{rrrrrrrl}
$t_{0}$ & $t_{1}$ & $x$ & $x_{2}$ & $y_{0}$ & $y_{1}$ & $z$ & \\
\cline { 1 - 6 } 1 & 1 & -1 & 0 & 0 & 0 & 0 & $L$ \\
0 & 0 & 1 & 1 & -1 & -1 & 0 & $M$ \\
0 & 0 & 0 & 0 & 1 & 1 & 1 & $N$
\end{tabular}

and Nef $F=\langle L, M, N\rangle$. The secondary fan has four maximal cones, as in Figure 2. We have:

- $-K_{F}=L+3 N$ is nef and big but not ample.

- $\quad X \sim 2 N$ is nef and big but not ample.

- $-\left(K_{F}+X\right) \sim L+N$ is nef and big but not ample.

The two constructions coincide Recall $^{15}$ that $\mathrm{MM}_{2-18}$ is a member of $|N|$ in the toric variety $G$ with weight data

\begin{tabular}{rrrrrrr}
$x_{0}$ & $x_{1}$ & $x_{2}$ & $y_{0}$ & $y_{1}$ & $z$ & \\
\cline { 1 - 4 } 1 & 1 & 1 & -1 & -1 & 0 & $M$ \\
0 & 0 & 0 & 1 & 1 & 1 & $N$
\end{tabular}

and Nef $G=\langle M, N\rangle$. The unstable locus is $\left(x_{0}=x_{1}=x_{2}=0\right) \cup\left(y_{0}=y_{1}=z=0\right)$. The linear system $|M|=\left|x_{0}, x_{1}, x_{2}\right|$ manifestly defines a morphism $G \rightarrow \mathbb{P}_{x_{0}, x_{1}, x_{2}}^{2}$ with fibre $\mathbb{P}^{2}$. If $F$ is the blow-up of $G$ along $\left(x_{0}=x_{1}=0\right)$ then $X$ is the proper transform of $\mathrm{MM}_{2-18}$. It is clear that $F$ is a toric variety with the weight data given above and that the morphism $F \rightarrow G$ is given by $x_{0}=x t_{0}, x_{1}=x t_{1}$.

${ }^{15}$ The description here differs from the weight data $\mathrm{MM}_{2-18}$ by a change of lattice basis and by relabelling of co-ordinates. 
The quantum period Let $p_{1}, p_{2}, p_{3} \in H^{\bullet}(F ; \mathbb{Z})$ denote the first Chern classes of $L, M$ and $N$, respectively; these classes form a basis for $H^{2}(F ; \mathbb{Z})$. Write $\tau \in H^{2}(F ; \mathbb{Q})$ as $\tau=\tau_{1} p_{1}+\tau_{2} p_{2}+\tau_{3} p_{3}$ and identify the group ring $\mathbb{Q}\left[H_{2}(F ; \mathbb{Z})\right]$ with the polynomial ring $\mathbb{Q}\left[Q_{1}, Q_{2}, Q_{3}\right]$ via the $\mathbb{Q}$-linear map that sends the element $Q^{\beta} \in \mathbb{Q}\left[H_{2}(F ; \mathbb{Z})\right]$ to $Q_{1}^{\left\langle\beta, p_{1}\right\rangle} Q_{2}^{\left\langle\beta, p_{2}\right\rangle} Q_{3}^{\left\langle\beta, p_{3}\right\rangle}$. We have

$$
\begin{aligned}
& I_{F}(\tau)=e^{\tau / z} \sum_{l, m, n \geq 0} \frac{Q_{1}^{l} Q_{2}^{m} Q_{3}^{n} e^{l \tau_{1}} e^{m \tau_{2}} e^{n \tau_{3}}}{\prod_{k=1}^{l}\left(p_{1}+k z\right)^{2} \prod_{k=1}^{m}\left(p_{2}+k z\right) \prod_{k=1}^{n}\left(p_{3}+k z\right)} \\
& \times \frac{\prod_{k=-\infty}^{0}\left(p_{2}-p_{1}+k z\right)}{\prod_{k=-\infty}^{m-l}\left(p_{2}-p_{1}+k z\right)} \frac{\prod_{k=-\infty}^{0}\left(p_{3}-p_{2}+k z\right)^{2}}{\prod_{k=-\infty}^{n-m}\left(p_{3}-p_{2}+k z\right)^{2}} .
\end{aligned}
$$

Since

$$
I_{F}(\tau)=1+\tau z^{-1}+O\left(z^{-2}\right),
$$

Theorem C.1 gives

$$
J_{F}(\tau)=I_{F}(\tau)
$$

We now proceed exactly as in the case of $\mathrm{MM}_{3-1}$, obtaining

$$
G_{X}(t)=e^{-4 t} \sum_{l=0}^{\infty} \sum_{n=0}^{\infty} \sum_{m=l}^{n} t^{l+n} \frac{(2 n) !}{(l !)^{2} m ! n !(m-l) !((n-m) !)^{2}} .
$$

Regularizing gives

$$
\begin{aligned}
\widehat{G}_{X}(t)=1+24 t^{2}+156 t^{3}+ & 2280 t^{4}+27960 t^{5}+387060 t^{6} \\
& +5450760 t^{7}+79246440 t^{8}+1175608560 t^{9}+\cdots .
\end{aligned}
$$

Minkowski period sequence 142

\section{The Fano manifold $\mathrm{MM}_{3-5}$}

Mori-Mukai construction The blow-up of $\mathbb{P}^{1} \times \mathbb{P}^{2}$ with centre a curve $C$ of bidegree $(5,2)$ such that the composition $C \hookrightarrow \mathbb{P}^{1} \times \mathbb{P}^{2} \rightarrow \mathbb{P}^{2}$ with projection to the second factor is an embedding.

Our construction A codimension-2 complete intersection $X$ of type

$$
(M+N) \cap(M+N)
$$


in the toric variety $F$ with weight data

\begin{tabular}{ccccccccc}
$t_{0}$ & $t_{1}$ & $y_{0}$ & $y_{1}$ & $y_{2}$ & $x$ & $x_{0}$ & $x_{1}$ & \\
\cline { 1 - 6 } 1 & 1 & 0 & 0 & 0 & -1 & 0 & 0 & $L$ \\
0 & 0 & 1 & 1 & 1 & -1 & 0 & 0 & $M$ \\
0 & 0 & 0 & 0 & 0 & 1 & 1 & 1 & $N$
\end{tabular}

and Nef $F=\langle L, M, N\rangle$. The secondary fan for $F$ is the same as for the toric variety in Section 55 (ie our ambient toric variety for $\mathrm{MM}_{3-2}$ ) and is in Figure 1. We have:

- $-K_{F}=L+2 M+3 N$ is ample, that is $F$ is a Fano variety.

- $X$ is complete intersection of two nef divisors on $F$.

- $-\left(K_{F}+\Lambda\right)=L+N$ is nef and big but not ample on $F$.

The two constructions coincide Apply Lemma E. 1 with $G=\mathbb{P}^{1} \times \mathbb{P}^{2}$ and

$$
\begin{aligned}
V & =\mathcal{O}_{\mathbb{P}^{1} \times \mathbb{P}^{2}}(-1,-1) \oplus \mathcal{O}_{\mathbb{P}^{1} \times \mathbb{P}^{2}} \oplus \mathcal{O}_{\mathbb{P}^{1} \times \mathbb{P}^{2},} \\
W & =\mathcal{O}_{\mathbb{P}^{1} \times \mathbb{P}^{2}}(0,1) \oplus \mathcal{O}_{\mathbb{P}^{1} \times \mathbb{P}^{2}}(0,1),
\end{aligned}
$$

with $f: V \rightarrow W$ given by the matrix

$$
\left(\begin{array}{lll}
t_{0} A_{2}(y) & y_{0} & y_{1} \\
t_{1} B_{2}(y) & y_{1} & y_{2}
\end{array}\right)
$$

where $\left[t_{0}: t_{1}\right]$ are homogeneous co-ordinates on $\mathbb{P}^{1}$ and $\left[y_{0}: y_{1}: y_{2}\right]$ are homogeneous co-ordinates on $\mathbb{P}^{2}$. This exhibits $X$ as the blow-up of $\mathbb{P}^{1} \times \mathbb{P}^{2}$ in the locus $Z$ defined by the condition

$$
\operatorname{rk}\left(\begin{array}{lll}
t_{0} A_{2}(y) & y_{0} & y_{1} \\
t_{1} B_{2}(y) & y_{1} & y_{2}
\end{array}\right)<2
$$

and it is easy to see that $C$ is described in this way. For instance, it is immediate that $Z$ projects isomorphically to a conic in $\mathbb{P}^{2}$ and that the projection to $\mathbb{P}^{1}$ has degree 5 .

The quantum period We proceed as in Example D.8. Let $p_{1}, p_{2}, p_{3} \in H^{\bullet}(F ; \mathbb{Z})$ denote the first Chern classes of $L, M$ and $N$, respectively; these classes form a basis for $H^{2}(F ; \mathbb{Z})$. Write $\tau \in H^{2}(F ; \mathbb{Q})$ as $\tau=\tau_{1} p_{1}+\tau_{2} p_{2}+\tau_{3} p_{3}$ and identify the group ring $\mathbb{Q}\left[H_{2}(F ; \mathbb{Z})\right]$ with the polynomial ring $\mathbb{Q}\left[Q_{1}, Q_{2}, Q_{3}\right]$ via the $\mathbb{Q}$-linear map that sends the element $Q^{\beta} \in \mathbb{Q}\left[H_{2}(F ; \mathbb{Z})\right]$ to $Q_{1}^{\left\langle\beta, p_{1}\right\rangle} Q_{2}^{\left\langle\beta, p_{2}\right\rangle} Q_{3}^{\left\langle\beta, p_{3}\right\rangle}$. Theorem C.1 gives

$$
\begin{array}{r}
J_{F}(\tau)=e^{\tau / z} \sum_{l, m, n \geq 0} \frac{Q_{1}^{l} Q_{2}^{m} Q_{3}^{n} e^{l \tau_{1}} e^{m \tau_{2}} e^{n \tau_{3}}}{\prod_{k=1}^{l}\left(p_{1}+k z\right)^{2} \prod_{k=1}^{m}\left(p_{2}+k z\right)^{3} \prod_{k=1}^{n}\left(p_{3}+k z\right)^{2}} \\
\times \frac{\prod_{k=-\infty}^{0}\left(p_{3}-p_{1}-p_{2}+k z\right)}{\prod_{k=-\infty}^{n-l-m}\left(p_{3}-p_{1}-p_{2}+k z\right)}
\end{array}
$$


and hence

$$
\begin{array}{r}
I_{\boldsymbol{e}, E}(\tau)=e^{\tau / z} \sum_{l, m, n \geq 0} \frac{Q_{1}^{l} Q_{2}^{m} Q_{3}^{n} e^{l \tau_{1}} e^{m \tau_{2}} e^{n \tau_{3}} \prod_{k=1}^{m+n}\left(\lambda+p_{2}+p_{3}+k z\right)^{2}}{\prod_{k=1}^{l}\left(p_{1}+k z\right)^{2} \prod_{k=1}^{m}\left(p_{2}+k z\right)^{3} \prod_{k=1}^{n}\left(p_{3}+k z\right)^{2}} \\
\times \frac{\prod_{k=-\infty}^{0}\left(p_{3}-p_{2}-p_{1}+k z\right)}{\prod_{k=-\infty}^{n-l-m}\left(p_{3}-p_{2}-p_{1}+k z\right)} .
\end{array}
$$

Note that

$$
I_{\boldsymbol{e}, E}(0)=A+B z^{-1}+O\left(z^{-2}\right),
$$

where

$$
\begin{aligned}
A=1 \quad \text { and } \quad B & =\left(Q_{3}+4 Q_{2} Q_{3}\right) 1+\left(p_{3}-p_{2}-p_{1}\right) \sum_{m>0} \frac{(-1)^{m-1} Q_{2}^{m}}{m} \\
& =Q_{3}\left(1+4 Q_{2}\right) 1+\left(p_{3}-p_{2}-p_{1}\right) \log \left(1+Q_{2}\right) .
\end{aligned}
$$

Arguing exactly as in Example D.8, we find that

$$
J_{\boldsymbol{e}, E}\left(\left(p_{3}-p_{2}-p_{1}\right) \log \left(1+Q_{2}\right)\right)=e^{-Q_{3}\left(1+4 Q_{2}\right) / z} I_{\boldsymbol{e}, E}(0)
$$

and

$$
\begin{aligned}
& J_{\boldsymbol{e}, E}\left(\left(p_{3}-p_{2}-p_{1}\right) \log \left(1+Q_{2}\right)\right) \\
& =e^{\left(p_{3}-p_{2}-p_{1}\right) \log \left(1+Q_{2}\right) / z}\left[J_{\boldsymbol{e}, E}(0)\right]_{Q_{1}}=\frac{Q_{1}}{1+Q_{2}}, Q_{2}=\frac{Q_{2}}{1+Q_{2}}, Q_{3}=Q_{3}\left(1+Q_{2}\right)
\end{aligned}
$$

Hence, using the inverse mirror map (9), we have

$$
\begin{aligned}
J_{\boldsymbol{e}, E}(0)= & {\left[e^{-\left(p_{3}-p_{2}-p_{1}\right) \log \left(1+Q_{2}\right) / z}\right.} \\
& \left.\quad \times J_{\boldsymbol{e}, E}\left(\left(p_{3}-p_{2}-p_{1}\right) \log \left(1+Q_{2}\right)\right)\right]_{Q_{1}=\frac{Q_{1}}{1-Q_{2}}, Q_{2}=\frac{Q_{2}}{1-Q_{2}}, Q_{3}=Q_{3}\left(1-Q_{2}\right)} \\
= & e^{\left(p_{3}-p_{2}-p_{1}\right) \log \left(1-Q_{2}\right) / z} \\
& \quad \times\left[e^{-Q_{3}\left(1+4 Q_{2}\right) / z} I_{\boldsymbol{e}, E}(0)\right]_{Q_{1}=\frac{Q_{1}}{1-Q_{2}}, Q_{2}=\frac{Q_{2}}{1-Q_{2}}, Q_{3}=Q_{3}\left(1-Q_{2}\right)}
\end{aligned}
$$

Taking the non-equivariant limit yields

$$
\begin{array}{r}
J_{F, X}(0)=e^{\left(p_{3}-p_{2}-p_{1}\right) \log \left(1-Q_{2}\right) / z} e^{-Q_{3}\left(1+3 Q_{2}\right)} \\
\times \sum_{l, m, n \geq 0} \frac{Q_{1}^{l} Q_{2}^{m} Q_{3}^{n}\left(1-Q_{2}\right)^{n-l-m} \prod_{k=1}^{m+n}\left(p_{2}+p_{3}+k z\right)^{2}}{\prod_{k=1}^{l}\left(p_{1}+k z\right)^{2} \prod_{k=1}^{m}\left(p_{2}+k z\right)^{3} \prod_{k=1}^{n}\left(p_{3}+k z\right)^{2}} \\
\times \frac{\prod_{k=-\infty}^{0}\left(p_{3}-p_{2}-p_{1}+k z\right)}{\prod_{k=-\infty}^{n-l-m}\left(p_{3}-p_{2}-p_{1}+k z\right)} .
\end{array}
$$


Recall that the quantum period $G_{X}$ is obtained from the component of $J_{X}(0)$ along the unit class $1 \in H^{\bullet}(X ; \mathbb{Q})$ by setting $z=1$ and $Q^{\beta}=t^{\left\langle\beta,-K_{X}\right\rangle}$. In view of (8), therefore, to obtain $G_{X}$ we extract the component of $J_{F, X}(0)$ along the unit class $1 \in H^{\bullet}(Y ; \mathbb{Q})$ and set $z=Q_{2}=1$ and $Q_{1}=Q_{3}=t$. This gives

Regularizing gives

$$
G_{X}(t)=e^{-4 t} \sum_{l=0}^{\infty} \sum_{m=0}^{\infty} t^{2 l+m} \frac{(l+2 m) !(l+2 m) !}{(l !)^{2}(m !)^{3}((l+m) !)^{2}} .
$$

$$
\begin{aligned}
\widehat{G}_{X}(t)=1+22 t^{2}+126 t^{3}+1722 t^{4}+ & 18780 t^{5}+236470 t^{6} \\
+ & 2998380 t^{7}+39440170 t^{8}+528743880 t^{9}+\cdots
\end{aligned}
$$

Minkowski period sequence 138

\section{The Fano manifold $\mathrm{MM}_{3-6}$}

Mori-Mukai construction The blow-up of $\mathbb{P}^{3}$ with centre a disjoint union of a line and an elliptic curve of degree 4 .

Our construction A member $X$ of $|2 M+N|$ in the toric variety with weight data

\begin{tabular}{cccccccc}
$s_{0}$ & $s_{1}$ & $x$ & $x_{2}$ & $x_{3}$ & $y_{0}$ & $y_{1}$ & \\
\cline { 1 - 6 } 1 & 1 & -1 & 0 & 0 & 0 & 0 & $L$ \\
0 & 0 & 1 & 1 & 1 & 0 & 0 & $M$ \\
0 & 0 & 0 & 0 & 0 & 1 & 1 & $N$
\end{tabular}

and Nef $F=\langle L, M, N\rangle$. The secondary fan for $F$ has two maximal cones, as in Figure 3.

We have:

- $-K_{F}=L+3 M+2 N$ is ample, that is, $F$ is a Fano variety.

- $X \sim 2 M+N$ is nef.

- $-\left(K_{F}+X\right) \sim L+M+N$ is ample.

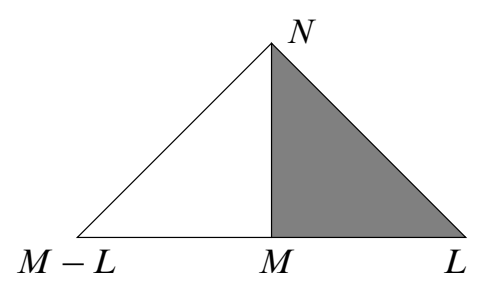

Figure 3: The secondary fan for $F$ in $\mathrm{MM}_{3-6}$. 
The two constructions coincide An elliptic curve $\Gamma \subset \mathbb{P}^{3}$ is a $(2,2)$-complete intersection in $\mathbb{P}^{3}$, so $X$ is constructed by applying Lemma E. 1 twice. In more detail, the equation of $X$ has the form

$$
y_{0} A\left(s_{0} x, s_{1} x, x_{2}, x_{3}\right)+y_{1} B\left(s_{0} x, s_{1} x, x_{2}, x_{3}\right)=0,
$$

where $A$ and $B$ are homogeneous quadratic polynomials in the variables $x_{0}=s_{0} x$, $x_{1}=s_{1} x, x_{2}$ and $x_{3}$. The obvious morphism $X \rightarrow \mathbb{P}_{x_{0}, x_{1}, x_{2}, x_{3}}^{3}$ blows up the line $x_{0}=x_{1}=0$ and the elliptic curve $A=B=0$.

The quantum period Corollary D.5 yields

and regularizing gives

$$
G_{X}(t)=e^{-3 t} \sum_{l=0}^{\infty} \sum_{n=0}^{\infty} \sum_{m=l}^{\infty} t^{l+m+n} \frac{(2 m+n) !}{(l !)^{2}(m-l) !(m !)^{2}(n !)^{2}}
$$

$$
\begin{aligned}
\widehat{G}_{X}(t)=1+14 t^{2}+66 t^{3}+762 t^{4}+ & 6960 t^{5}+73490 t^{6} \\
& +780360 t^{7}+8578570 t^{8}+96096000 t^{9}+\cdots .
\end{aligned}
$$

Minkowski period sequence 117

\section{The Fano manifold $\mathrm{MM}_{3-7}$}

Mori-Mukai construction The blow-up of $W \subset \mathbb{P}^{2} \times \mathbb{P}^{2}$ with centre an elliptic curve which is an intersection of two members of $\left|-\frac{1}{2} K_{W}\right|$. Here $W$ is a divisor of bidegree $(1,1)$ in $\mathbb{P}^{2} \times \mathbb{P}^{2}$.

Our construction A complete intersection $X$ of type $(M+N) \cap(L+M+N)$ in the toric variety $F=\mathbb{P}^{1} \times \mathbb{P}^{2} \times \mathbb{P}^{2}$.

The two constructions coincide Apply Lemma E.1.

The quantum period The toric variety $F$ has weight data

$\begin{array}{lllllllll}1 & 1 & 0 & 0 & 0 & 0 & 0 & 0 & L \\ 0 & 0 & 1 & 1 & 1 & 0 & 0 & 0 & M \\ 0 & 0 & 0 & 0 & 0 & 1 & 1 & 1 & N\end{array}$

and Nef $F=\langle L, M, N\rangle$. We have:

- $F$ is a Fano variety.

- $X$ is the complete intersection of two nef divisors on $F$. 
- $-\left(K_{F}+\Lambda\right)=L+M+N$ is ample on $F$.

Corollary D.5 yields

and regularizing gives

$$
G_{X}(t)=e^{-3 t} \sum_{l=0}^{\infty} \sum_{m=0}^{\infty} \sum_{n=0}^{\infty} t^{l+m+n} \frac{(l+m+n) !(m+n) !}{(l !)^{2}(m !)^{3}(n !)^{3}}
$$

$$
\begin{aligned}
\widehat{G}_{X}(t)=1+10 t^{2}+48 t^{3}+438 t^{4}+ & 3720 t^{5}+33940 t^{6} \\
& +320040 t^{7}+3096310 t^{8}+30581040 t^{9}+\cdots .
\end{aligned}
$$

Minkowski period sequence 103

\section{The Fano manifold $\mathrm{MM}_{3-8}$}

Mori-Mukai construction A member of the linear system $\left|p_{1}^{\star} g^{\star} \mathcal{O}(1) \otimes p_{2}^{\star} \mathcal{O}(2)\right|$ on $\mathbb{F}_{1} \times \mathbb{P}^{2}$, where $p_{i}(i=1,2)$ is the projection to the $i^{\text {th }}$ factor and $g: \mathbb{F}_{1} \rightarrow \mathbb{P}^{2}$ is the blow-up.

Our construction A member $X$ of $|M+2 N|$ in the toric variety $F$ with weight data

\begin{tabular}{cccccccc}
$s_{0}$ & $s_{1}$ & $x$ & $x_{2}$ & $y_{0}$ & $y_{1}$ & $y_{2}$ & \\
\cline { 1 - 6 } 1 & 1 & -1 & 0 & 0 & 0 & 0 & $L$ \\
0 & 0 & 1 & 1 & 0 & 0 & 0 & $M$ \\
0 & 0 & 0 & 0 & 1 & 1 & 1 & $N$
\end{tabular}

and Nef $F=\langle L, M, N\rangle$. The secondary fan for $F$ is the same as for the toric variety in Section 59 (ie our ambient toric variety for $\mathrm{MM}_{3-6}$ ) and is in Figure 3. We have:

- $-K_{F}=L+2 M+3 N$ is ample, that is, $F$ is a Fano variety.

- $\quad X \sim M+2 N$ is nef.

- $-\left(K_{F}+X\right) \sim L+M+N$ is ample.

The two constructions coincide Obvious.

The quantum period Corollary D.5 yields

and regularizing gives

$$
G_{X}(t)=e^{-3 t} \sum_{l=0}^{\infty} \sum_{m=l}^{\infty} \sum_{n=0}^{\infty} t^{l+m+n} \frac{(m+2 n) !}{(l !)^{2}(m-l) ! m !(n !)^{3}}
$$

$$
\begin{aligned}
\widehat{G}_{X}(t)=1+12 t^{2}+54 t^{3}+540 t^{4}+ & 4620 t^{5}+43770 t^{6} \\
& +425880 t^{7}+4256700 t^{8}+43462440 t^{9}+\cdots .
\end{aligned}
$$




\section{Minkowski period sequence 112}

\section{The Fano manifold $\mathrm{MM}_{3-9}$}

Mori-Mukai construction The blow-up of the cone $W_{4} \subset \mathbb{P}^{6}$ over the Veronese surface $R_{4} \subset \mathbb{P}^{5}$ with centre a disjoint union of the vertex and a quartic in $R_{4} \cong \mathbb{P}^{2}$.

Our construction A member $X$ of $|2 M|$ in the toric variety $F$ with weight data

\begin{tabular}{ccccccc}
$s_{0}$ & $s_{1}$ & $s_{2}$ & $x$ & $y_{0}$ & $y_{1}$ & \\
\cline { 1 - 5 } 1 & 1 & 1 & -2 & 0 & 0 & $L$ \\
0 & 0 & 0 & 1 & 1 & 1 & $M$
\end{tabular}

and Nef $F=\langle L, M\rangle$.

We have:

- $-K_{F}=L+3 M$ is ample, so $F$ is a Fano variety.

- $X \sim 2 M$ is nef.

- $-\left(K_{F}+X\right) \sim L+M$ is ample.

The two constructions coincide The variety $X$ is cut out by

$$
y_{0} y_{1}+x^{2} A_{4}\left(s_{0}, s_{1}, s_{2}\right)=0,
$$

where $A_{4}$ is a generic homogeneous polynomial of degree 4 in $s_{0}, s_{1}$ and $s_{2}$. Note the morphisms $\pi: F \rightarrow \mathbb{P}^{2}$, given by the linear system $|L|$, and $f: F \rightarrow \mathbb{P}(1,1,1,2,2)$, given (contravariantly) by $\left[x_{0}, x_{1}, x_{2}, y_{0}, y_{1}\right] \mapsto\left[s_{0} \sqrt{x}, s_{1} \sqrt{x}, s_{2} \sqrt{x}, y_{0}, y_{1}\right]$. The exceptional set of $f$ is the divisor $E=(x=0)=\mathbb{P}_{s_{0}, s_{1}, s_{2}}^{2} \times \mathbb{P}_{y_{0}, y_{1}}^{1}$ that maps to $\mathbb{P}_{y_{0}, y_{1}}^{1} \subset \mathbb{P}(1,1,1,2,2)$. Note that $E \cap X$ is $t w o$ copies of $\mathbb{P}^{2}$, one above $\left[y_{0}: y_{1}\right]=[1: 0]$ and one above $\left[y_{0}: y_{1}\right]=[0: 1]$. This explains how $X$ has rank 3 when $F$ has rank 2 . To see that our construction coincides with the construction of Mori and Mukai, set $W=f(X)$, note that

$$
W=\left(y_{0} y_{1}+A_{4}\left(x_{0}, x_{1}, x_{2}\right)=0\right) \subset \mathbb{P}(1,1,1,2,2)
$$

and note that the morphism $f: X \rightarrow W$ contracts one copy of $\mathbb{P}^{2}$, with normal bundle $\mathcal{O}(-2)$, to each of the two singular points $W \cap \mathbb{P}_{y_{0}, y_{1}}^{1}$. Consider the rational projection

$$
g: \mathbb{P}(1,1,1,2,2) \rightarrow \mathbb{P}(1,1,1,2)_{x_{0}, x_{1}, x_{2}, y_{0}}
$$

which omits the homogeneous co-ordinate $y_{1}$. It is clear that $\left.g\right|_{W}: W \rightarrow \mathbb{P}(1,1,1,2)$ extends to a morphism after blowing up the singular point $[0: 0: 0: 0: 1] \in W$, and that this morphism contracts the surface $\left(y_{0}=A_{4}\left(x_{0}, x_{1}, x_{2}\right)=0\right) \subset W$ to the curve $\left(y_{0}=A_{4}\left(x_{0}, x_{1}, x_{2}\right)=0\right) \subset \mathbb{P}(1,1,1,2)$. 
The quantum period Corollary D.5 yields

and regularizing gives

$$
G_{X}(t)=e^{-2 t} \sum_{l=0}^{\infty} \sum_{m=2 l}^{\infty} t^{l+m} \frac{(2 m) !}{(l !)^{3}(m-2 l) !(m !)^{2}}
$$

$$
\begin{aligned}
\widehat{G}_{X}(t)=1+2 t^{2}+36 t^{3}+198 t^{4}+840 t^{5} & +9200 t^{6} \\
& +79800 t^{7}+520870 t^{8}+4289040 t^{9}+\cdots .
\end{aligned}
$$

Minkowski period sequence 22

\section{The Fano manifold $M_{3-10}$}

Mori-Mukai construction The blow-up of a quadric 3-fold $Q \subset \mathbb{P}^{4}$ with centre a disjoint union of two conics on it.

Our construction A member $X$ of $|2 N|$ in the toric variety $F$ with weight data

\begin{tabular}{rrrrrrrr}
$s_{0}$ & $s_{1}$ & $t_{2}$ & $t_{3}$ & $x$ & $y$ & $x_{4}$ & \\
\cline { 1 - 6 } 1 & 1 & 0 & 0 & -1 & 0 & 0 & $L$ \\
0 & 0 & 1 & 1 & 0 & -1 & 0 & $M$ \\
0 & 0 & 0 & 0 & 1 & 1 & 1 & $N$
\end{tabular}

and Nef $F=\langle L, M, N\rangle$. The secondary fan for $F$ has 4 maximal cones, as in Figure 4. We have:

- $-K_{F}=L+M+3 N$ is ample, so that $F$ is a Fano variety.

- $\quad X \sim 2 N$ is nef.

- $-\left(K_{F}+X\right) \sim L+M+N$ is ample.

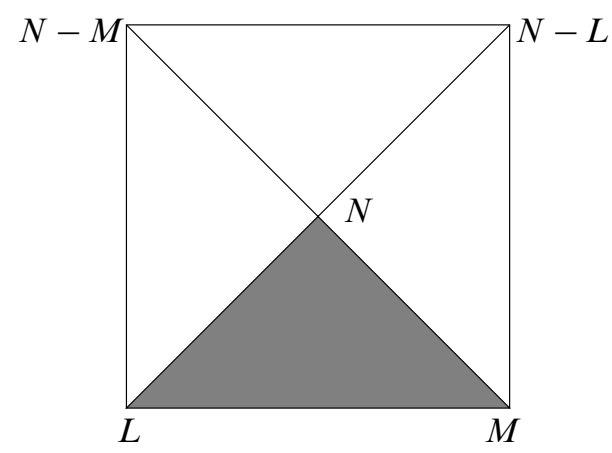

Figure 4: The secondary fan for $F$ in $\mathrm{MM}_{3-10}$. 
The two constructions coincide We take $Q$ to be the locus $x_{0} x_{1}+x_{2} x_{3}+x_{4}^{2}=0$ in $\mathbb{P}_{x_{0}, x_{1}, x_{2}, x_{3}, x_{4}}^{4}$ and take the conics to be cut out of $Q$ by the two complete intersections $\left(x_{0}=x_{1}=0\right)$ and $\left(x_{2}=x_{3}=0\right)$; note that the intersection of these two planes misses $Q$. The morphism $F \rightarrow \mathbb{P}^{4}$ given (contravariantly) by

$$
\left[x_{0}: x_{1}: x_{2}: x_{3}: x_{4}\right] \mapsto\left[s_{0} x: s_{1} x: t_{2} y: t_{3} y: x_{4}\right]
$$

blows up the planes $\left(x_{0}=x_{1}=0\right)$ and $\left(x_{2}=x_{3}=0\right)$. Taking the proper transform of $Q$ yields $X$.

The quantum period Corollary D.5 yields

$$
G_{X}(t)=e^{-2 t} \sum_{l=0}^{\infty} \sum_{m=0}^{\infty} \sum_{n=\max (l, m)}^{\infty} t^{l+m+n} \frac{(2 n) !}{(l !)^{2}(m !)^{2} n !(n-l) !(n-m) !}
$$

and regularizing gives

$$
\begin{aligned}
\widehat{G}_{X}(t)=1+10 t^{2}+36 t^{3}+366 t^{4}+ & 2640 t^{5}+23320 t^{6} \\
& +200760 t^{7}+1815310 t^{8}+16611840 t^{9}+\cdots .
\end{aligned}
$$

Minkowski period sequence 99

\section{The Fano manifold $M_{3-11}$}

Mori-Mukai construction The blow-up of $B_{7}\left(\right.$ see $\left.\mathrm{MM}_{2-35}\right)$ with centre an elliptic curve that is the intersection of two members of $\left|-\frac{1}{2} K_{B_{7}}\right|$.

Our construction A member $X$ of $|L+M+N|$ in the toric variety $F$ with weight data

\begin{tabular}{cccrcccc}
$s_{0}$ & $s_{1}$ & $s_{2}$ & $x$ & $x_{3}$ & $y_{0}$ & $y_{1}$ & \\
\cline { 1 - 6 } 1 & 1 & 1 & -1 & 0 & 0 & 0 & $L$ \\
0 & 0 & 0 & 1 & 1 & 0 & 0 & $M$ \\
0 & 0 & 0 & 0 & 0 & 1 & 1 & $N$
\end{tabular}

and Nef $F=\langle L, M, N\rangle$. In other words, $F \cong B_{7} \times \mathbb{P}^{1}$. The secondary fan of $F$ is the same as that of the toric variety in Section 59 (ie our ambient toric variety for $\mathrm{MM}_{3-6}$ ) and is shown in Figure 3 .

We have:

- $-K_{F}=2 L+2 M+2 N$ is ample, so $F$ is a Fano variety.

- $X \sim L+M+N$ is ample.

- $-\left(K_{F}+X\right) \sim L+M+N$ is ample. 
The two constructions coincide Recall that $B_{7}\left(\mathrm{MM}_{2-35}\right)$ is the toric variety with weight data

\begin{tabular}{rrrrrr}
$s_{0}$ & $s_{1}$ & $s_{2}$ & $x$ & $x_{3}$ & \\
\cline { 1 - 4 } 1 & 1 & 1 & -1 & 0 & $L$ \\
0 & 0 & 0 & 1 & 1 & $M$
\end{tabular}

and Nef $B_{7}=\langle L, M\rangle$. Now apply Lemma E.1 with $V=\mathcal{O}_{B_{7}} \oplus \mathcal{O}_{B_{7}}, W=-\frac{1}{2} K_{B_{7}}$ and $f: V \rightarrow W$ given by $(A B)$, where $A$ and $B$ are the sections of $-\frac{1}{2} K_{B_{7}}$ that define the centre of the blow-up.

The quantum period Corollary D.5 yields

and regularizing gives

$$
G_{X}(t)=e^{-2 t} \sum_{l=0}^{\infty} \sum_{n=0}^{\infty} \sum_{m=l}^{\infty} t^{l+m+n} \frac{(l+m+n) !}{(l !)^{3}(m-l) ! m !(n !)^{2}}
$$

$$
\begin{aligned}
\widehat{G}_{X}(t)=1+6 t^{2}+30 t^{3}+186 t^{4} & +1380 t^{5}+10230 t^{6} \\
& +78540 t^{7}+620970 t^{8}+5020680 t^{9}+\cdots .
\end{aligned}
$$

Minkowski period sequence 72

\section{The Fano manifold $\mathrm{MM}_{3-12}$}

Mori-Mukai construction The blow-up of $\mathbb{P}^{3}$ with centre a disjoint union of a line and a twisted cubic.

Our construction A codimension-2 complete intersection $X$ of type

$$
(M+N) \cap(M+N)
$$

in the toric variety $F$ with weight data

\begin{tabular}{ccccccccc}
$s_{0}$ & $s_{3}$ & $x$ & $x_{1}$ & $x_{2}$ & $y_{0}$ & $y_{1}$ & $y_{2}$ & \\
\cline { 1 - 7 } 1 & 1 & -1 & 0 & 0 & 0 & 0 & 0 & $L$ \\
0 & 0 & 1 & 1 & 1 & 0 & 0 & 0 & $M$ \\
0 & 0 & 0 & 0 & 0 & 1 & 1 & 1 & $N$
\end{tabular}

and Nef $F=\langle L, M, N\rangle$. The secondary fan of $F$ is the same as of the toric variety in Section 59 (ie our ambient toric variety for $\mathrm{MM}_{3-6}$ ) and is in Figure 3. We have:

- $-K_{F}=L+3 M+3 N$ is ample, so $F$ is a Fano variety.

- $X$ is the complete intersection of two nef divisors on $F$.

- $-\left(K_{F}+\Lambda\right) \sim L+M+N$ is ample. 
The two constructions coincide The twisted cubic $\Gamma$ is cut out of $\mathbb{P}_{x_{0}, \ldots, x_{3}}^{3}$ by the equations

$$
\operatorname{rk}\left(\begin{array}{lll}
x_{0} & x_{1} & x_{2} \\
x_{1} & x_{2} & x_{3}
\end{array}\right)<2
$$

By Lemma E. 1 the blow up of $\mathbb{P}^{3}$ along $\Gamma$ is cut out of $\mathbb{P}_{x_{0}, \ldots, x_{3}}^{3} \times \mathbb{P}_{y_{0}, y_{1}, y_{2}}^{2}$ by the equations

$$
\left(\begin{array}{lll}
x_{0} & x_{1} & x_{2} \\
x_{1} & x_{2} & x_{3}
\end{array}\right) \cdot\left(\begin{array}{l}
y_{0} \\
y_{1} \\
y_{2}
\end{array}\right)=0
$$

Observe that $\Gamma$ is disjoint from the line $\left(x_{0}=x_{3}=0\right)$. We therefore blow up $\mathbb{P}_{x_{0}, \ldots, x_{3}}^{3} \times \mathbb{P}_{y_{0}, y_{1}, y_{2}}^{2}$ along the locus $x_{0}=x_{3}=0$, obtaining the toric variety $F$. The equations defining $X$ inside $F$ are

$$
\left(\begin{array}{ccc}
s_{0} x & x_{1} & x_{2} \\
x_{1} & x_{2} & s_{3} x
\end{array}\right) \cdot\left(\begin{array}{l}
y_{0} \\
y_{1} \\
y_{2}
\end{array}\right)=0
$$

and so $X$ is a complete intersection of type $(M+N) \cap(M+N)$.

The quantum period Corollary D.5 yields

$$
G_{X}(t)=e^{-2 t} \sum_{l=0}^{\infty} \sum_{n=0}^{\infty} \sum_{m=l}^{\infty} t^{l+m+n} \frac{(m+n) !(m+n) !}{(l !)^{2}(m-l) !(m !)^{2}(n !)^{3}}
$$

and regularizing gives

$$
\begin{aligned}
\widehat{G}_{X}(t)=1+8 t^{2}+30 t^{3}+240 t^{4}+1740 t^{5}+13130 t^{6} \\
+106680 t^{7}+862960 t^{8}+7248360 t^{9}+\cdots .
\end{aligned}
$$

Minkowski period sequence 85

\section{The Fano manifold $M_{3-13}$}

Mori-Mukai construction The blow-up of $W \subset \mathbb{P}^{2} \times \mathbb{P}^{2}$ with centre a curve $C$ of bidegree $(2,2)$ on it such that $C \hookrightarrow W \rightarrow \mathbb{P}^{2} \times \mathbb{P}^{2} \stackrel{p_{i}}{\longrightarrow} \mathbb{P}^{2}$ is an embedding for $i=1,2$. Here $W$ is a divisor of bidegree $(1,1)$ in $\mathbb{P}^{2} \times \mathbb{P}^{2}$ and $p_{i}: \mathbb{P}^{2} \times \mathbb{P}^{2} \rightarrow \mathbb{P}^{2}$ is projection to the $i^{\text {th }}$ factor.

Our construction A codimension- 3 complete intersection $X$ of type

$$
(L+M) \cap(L+N) \cap(M+N)
$$

in $\mathbb{P}^{2} \times \mathbb{P}^{2} \times \mathbb{P}^{2}$. 
The two constructions coincide First choose co-ordinates $x_{0}, x_{1}, x_{2}, y_{0}, y_{1}, y_{2}$ on $\mathbb{P}^{2} \times \mathbb{P}^{2}$ such that the curve $C$ is contained in the surface $\Sigma$ given by the condition

$$
\operatorname{rk}\left(\begin{array}{lll}
x_{0} & x_{1} & x_{2} \\
y_{0} & y_{1} & y_{2}
\end{array}\right)<2
$$

Note that $\Sigma$ is just $\mathbb{P}^{2}$ embedded diagonally in $\mathbb{P}^{2} \times \mathbb{P}^{2}$. In these co-ordinates, $W_{1,1}=\left\{f_{1,1}(x, y)=0\right\}$, where $f_{1,1} \in \Gamma\left(\mathbb{P}^{2} \times \mathbb{P}^{2} ; \mathcal{O}(1,1)\right)$ is a general section and $C=\Sigma \cdot W_{1,1}$. By Lemma E.1, $X$ is given by the equations

$$
\left\{\begin{aligned}
x_{0} z_{0}+x_{1} z_{1}+x_{2} z_{2} & =0 \\
y_{0} z_{0}+y_{1} z_{1}+y_{2} z_{2} & =0 \\
f_{1,1}(x, y) & =0
\end{aligned}\right.
$$

in $\mathbb{P}_{x_{0}, x_{1}, x_{2}}^{2} \times \mathbb{P}_{y_{0}, y_{1}, y_{2}}^{2} \times \mathbb{P}_{z_{0}, z_{1}, z_{2}}^{2}$.

The quantum period $F=\mathbb{P}^{2} \times \mathbb{P}^{2} \times \mathbb{P}^{2}$ is the toric variety with weight data

$$
\begin{array}{llllllllll}
1 & 1 & 1 & 0 & 0 & 0 & 0 & 0 & 0 & L \\
0 & 0 & 0 & 1 & 1 & 1 & 0 & 0 & 0 & M \\
0 & 0 & 0 & 0 & 0 & 0 & 1 & 1 & 1 & N
\end{array}
$$

and Nef $F=\langle L, M, N\rangle$. We have:

- $F$ is a Fano variety.

- $X$ is the complete intersection of three nef divisors on $F$.

- $-\left(K_{F}+\Lambda\right) \sim L+M+N$ is ample.

Corollary D.5 yields

$$
G_{X}(t)=e^{-3 t} \sum_{l=0}^{\infty} \sum_{m=0}^{\infty} \sum_{n=0}^{\infty} t^{l+m+n} \frac{(l+m) !(l+n) !(m+n) !}{(l !)^{3}(m !)^{3}(n !)^{3}}
$$

and regularizing gives

$$
\begin{aligned}
\widehat{G}_{X}(t)=1+6 t^{2}+24 t^{3}+162 t^{4} & +1080 t^{5} \\
& +7620 t^{6}+55440 t^{7}+415170 t^{8}+3166800 t^{9}+\cdots .
\end{aligned}
$$

Minkowski period sequence 70

\section{The Fano manifold $M_{3-14}$}

Mori-Mukai construction The blow-up of $\mathbb{P}^{3}$ with centre a union of a cubic in a plane $S$ and a point not in $S$. 


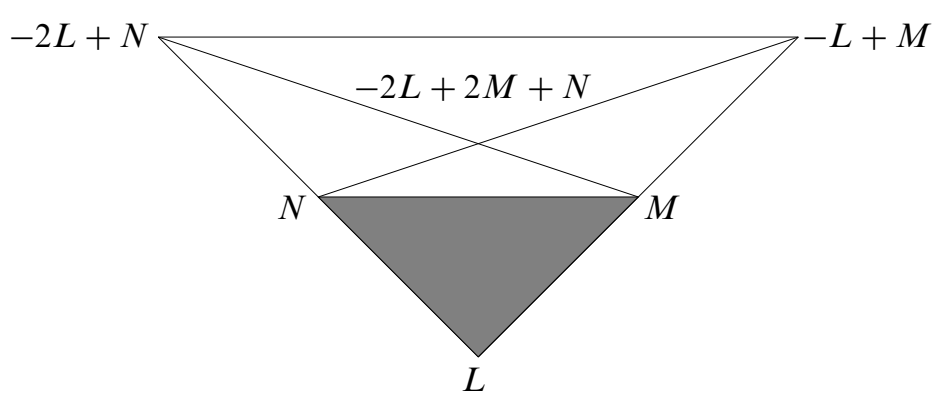

Figure 5: The secondary fan for $F$ in $\mathrm{MM}_{3-14}$.

Our construction A member $X$ of $|M+N|$ in the toric variety $F$ with weight data

\begin{tabular}{rrrrrrrr}
$s_{0}$ & $s_{1}$ & $s_{2}$ & $x$ & $x_{3}$ & $u$ & $v$ & \\
\cline { 1 - 5 } 1 & 1 & 1 & -1 & 0 & -2 & 0 & $L$ \\
0 & 0 & 0 & 1 & 1 & 0 & 0 & $M$ \\
0 & 0 & 0 & 0 & 0 & 1 & 1 & $N$
\end{tabular}

and Nef $F=\langle L, M, N\rangle$. The secondary fan of $F$ is shown in Figure 5.

We have:

- $-K_{F}=2 M+2 N$ is nef and big but not ample.

- $X \sim M+N$ is nef and big but not ample.

- $-\left(K_{F}+X\right) \sim M+N$ is nef and big but not ample.

The two constructions coincide The variety $X$ is cut out by

$$
v x_{3}+u x A_{3}\left(s_{0}, s_{1}, s_{2}\right)=0 .
$$

Note the obvious morphism $\pi: F \rightarrow B_{7}$ with fibre $\mathbb{P}_{u, v}^{1}$, where $B_{7}$ is the toric variety with weight data

\begin{tabular}{cccccc}
$s_{0}$ & $s_{1}$ & $s_{2}$ & $x$ & $x_{3}$ & \\
\hline 1 & 1 & 1 & -1 & 0 & $L$ \\
0 & 0 & 0 & 1 & 1 & $M$
\end{tabular}

and Nef $B_{7}=\langle L, M\rangle$. (The weight data and co-ordinates for $B_{7}$ here are exactly as in Section 52.) The birational morphism $B_{7} \rightarrow \mathbb{P}^{3}$ given (contravariantly) by $\left[x_{0}, \ldots, x_{3}\right] \mapsto\left[s_{0} x, s_{1} x, s_{2} x, x_{3}\right]$ identifies $B_{7}$ with the blow-up of $\mathbb{P}^{3}$ at the point $[0: 0: 0: 1]$. The equation defining $X$ is of degree 1 in $\mathbb{P}_{u, v}^{1}$; it follows that the morphism $\left.\pi\right|_{X}: X \rightarrow B_{7}$ is birational and blows up the $\operatorname{locus}^{16}\left(x_{3}=A_{3}\left(s_{0}, s_{1}, s_{2}\right)=0\right) \subset B_{7}$.

${ }^{16}$ With our choice of stability condition for $F,\left(x_{3}=x=0\right) \subset \mathbb{C}^{7}$ is part of the unstable locus. 
The quantum period Let $p_{1}, p_{2}, p_{3} \in H^{\bullet}(F ; \mathbb{Z})$ denote the first Chern classes of $L, M$ and $N$, respectively; these classes form a basis for $H^{2}(F ; \mathbb{Z})$. Write $\tau \in H^{2}(F ; \mathbb{Q})$ as $\tau=\tau_{1} p_{1}+\tau_{2} p_{2}+\tau_{3} p_{3}$ and identify the group ring $\mathbb{Q}\left[H_{2}(F ; \mathbb{Z})\right]$ with the polynomial ring $\mathbb{Q}\left[Q_{1}, Q_{2}, Q_{3}\right]$ via the $\mathbb{Q}$-linear map that sends the element $Q^{\beta} \in \mathbb{Q}\left[H_{2}(F ; \mathbb{Z})\right]$ to $Q_{1}^{\left\langle\beta, p_{1}\right\rangle} Q_{2}^{\left\langle\beta, p_{2}\right\rangle} Q_{3}^{\left\langle\beta, p_{3}\right\rangle}$. We have

$$
\begin{aligned}
& I_{F}(\tau)=e^{\tau / z} \sum_{l, m, n \geq 0} \frac{Q_{1}^{l} Q_{2}^{m} Q_{3}^{n} e^{l \tau_{1}} e^{m \tau_{2}} e^{n \tau_{3}}}{\prod_{k=1}^{l}\left(p_{1}+k z\right)^{3} \prod_{k=1}^{m}\left(p_{2}+k z\right) \prod_{k=1}^{n}\left(p_{3}+k z\right)} \\
& \times \frac{\prod_{k=-\infty}^{0}\left(p_{2}-p_{1}+k z\right)}{\prod_{k=-\infty}^{m-l}\left(p_{2}-p_{1}+k z\right)} \frac{\prod_{k=-\infty}^{0}\left(p_{3}-2 p_{1}+k z\right)^{2}}{\prod_{k=-\infty}^{n-2 l}\left(p_{3}-2 p_{1}+k z\right)^{2}} .
\end{aligned}
$$

Since

$$
I_{F}(\tau)=1+\tau z^{-1}+O\left(z^{-2}\right)
$$

Theorem C.1 gives

$$
J_{F}(\tau)=I_{F}(\tau) .
$$

We now proceed exactly as in the case of $\mathrm{MM}_{3-1}$, obtaining:

Regularizing gives

$$
G_{X}(t)=e^{-2 t} \sum_{l=0}^{\infty} \sum_{m=l}^{\infty} \sum_{n=2 l}^{\infty} t^{m+n} \frac{(m+n) !}{(l !)^{3} m ! n !(m-l) !(n-2 l) !}
$$

$\widehat{G}_{X}(t)=1+2 t^{2}+18 t^{3}+102 t^{4}+420 t^{5}+2810 t^{6}+21000 t^{7}+129430 t^{8}+813960 t^{9}+\cdots$.

\section{Minkowski period sequence 21}

\section{The Fano manifold $M_{3-15}$}

Mori-Mukai construction The blow-up of a quadric 3 -fold $Q \subset \mathbb{P}^{4}$ with centre a disjoint union of a line and a conic on it.

Our construction A member $X$ of $|L+N|$ in a toric variety $F$ with weight data

\begin{tabular}{rrrrrrrr}
$s_{0}$ & $s_{1}$ & $s_{2}$ & $t_{3}$ & $t_{4}$ & $y$ & $z$ & \\
\cline { 1 - 6 } 1 & 1 & 1 & 0 & 0 & -1 & 0 & $L$ \\
0 & 0 & 0 & 1 & 1 & 0 & -1 & $M$ \\
0 & 0 & 0 & 0 & 0 & 1 & 1 & $N$
\end{tabular}

and Nef $F=\langle L, M, N\rangle$. The secondary fan for $F$ is the same as that for the toric variety in Section 63 (ie our ambient toric variety for $\mathrm{MM}_{3-10}$ ) and is shown in Figure 4. We have: 
- $-K_{F}=2 L+M+2 N$ is ample, that is, $F$ is a Fano variety.

- $X \sim N+L$ is nef.

- $-\left(K_{F}+X\right) \sim L+M+N$ is ample on $F$.

The two constructions coincide The morphism $F \rightarrow \mathbb{P}^{4}$ given (contravariantly) by

$$
\left[x_{0}, x_{1}, x_{2}, x_{3}, x_{4}\right] \mapsto\left[s_{0} y, s_{1} y, s_{2} y, t_{3} z, t_{4} z\right]
$$

is the blow-up of $\mathbb{P}^{2}$ along the disjoint union of the line $\left(x_{0}=x_{1}=x_{2}=0\right)$ and the plane $\left(x_{3}=x_{4}=0\right) . X$ is the proper transform of the (nonsingular) quadric defined by the equation:

$$
x_{0}^{2}+x_{1} x_{3}+x_{2} x_{4}=0
$$

Note that this quadric contains the line $x_{0}=x_{1}=x_{2}=0$.

The quantum period Corollary D.5 yields

$$
G_{X}(t)=e^{-t} \sum_{l=0}^{\infty} \sum_{m=0}^{\infty} \sum_{n=\max (l, m)}^{\infty} t^{l+m+n} \frac{(l+n) !}{(l !)^{3}(m !)^{2}(n-l) !(n-m) !}
$$

and regularizing gives

$$
\begin{aligned}
\widehat{G}_{X}(t)=1+6 t^{2}+18 t^{3}+138 t^{4}+780 t^{5} & +5370 t^{6} \\
& +36120 t^{7}+253050 t^{8}+1811880 t^{9}+\cdots .
\end{aligned}
$$

Minkowski period sequence 67

\section{The Fano manifold $\mathrm{MM}_{3-16}$}

Mori-Mukai construction The blow-up of $B_{7}\left(\mathrm{MM}_{2-35}\right)$ with centre the strict transform of a twisted cubic passing through the centre of the blow-up $B_{7} \rightarrow \mathbb{P}^{3}$.

Our construction A complete intersection $X$ of type $N \cap N$ in the toric variety $F$ with weight data

\begin{tabular}{rrrrrrrrr}
$s_{1}$ & $s_{2}$ & $s_{3}$ & $x$ & $x_{0}$ & $y_{0}$ & $y_{1}$ & $y_{2}$ & \\
\cline { 1 - 3 } & 1 & 1 & -1 & 0 & 0 & -1 & -1 & \\
0 & 0 & 0 & 1 & 1 & -1 & 0 & 0 & $M$ \\
0 & 0 & 0 & 0 & 0 & 1 & 1 & 1 & $N$
\end{tabular}

and Nef $F=\langle L, M, N\rangle$. The secondary fan for $F$ is shown schematically in Figure 6 . We have: 


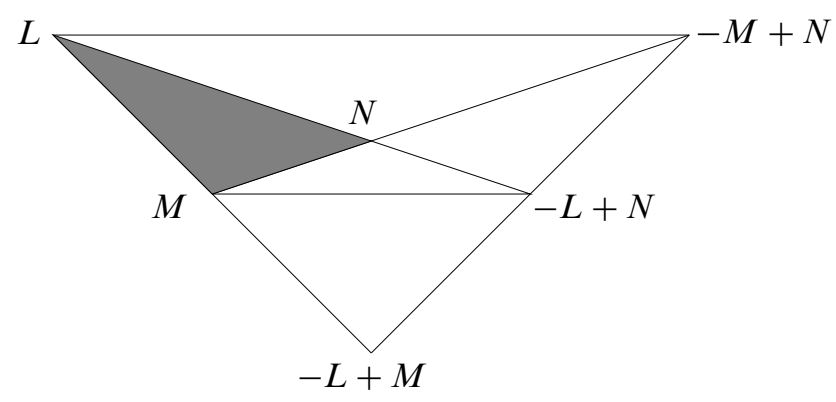

Figure 6: The secondary fan for $F$ in $\mathrm{MM}_{3-16}$.

- $-K_{F}=M+3 N$ is nef and big but not ample.

- $\quad X$ is the complete intersection of two nef divisors on $F$.

- $-\left(K_{F}+X\right) \sim M+N$ is nef and big but not ample on $F$.

The two constructions coincide Consider the rational normal curve

$$
\Gamma=\left\{\operatorname{rk}\left(\begin{array}{lll}
x_{0} & x_{1} & x_{2} \\
x_{1} & x_{2} & x_{3}
\end{array}\right)<2\right\}
$$

in $\mathbb{P}_{x_{0}, x_{1}, x_{2}, x_{3}}^{3}$ and note that $P=[1: 0: 0: 0]$ lies on $\Gamma$. Recall that $B_{7}$ is the toric variety with weight data

\begin{tabular}{cccccc}
$s_{1}$ & $s_{2}$ & $s_{3}$ & $x$ & $x_{0}$ & \\
\cline { 1 - 4 } 1 & 1 & 1 & -1 & 0 & $L$ \\
0 & 0 & 0 & 1 & 1 & $M$
\end{tabular}

and Nef $B_{7}=\langle L, M\rangle$, and that the blow-up morphism $B_{7} \rightarrow \mathbb{P}^{3}$ is given (contravariantly) by $\left[x_{0}, x_{1}, x_{2}, x_{3}\right] \mapsto\left[x_{0}, s_{1} x, s_{2} x, s_{3} x\right]$. The proper transform of the curve $\Gamma$ is the curve $\Gamma^{\prime}$ defined by the condition:

$$
\operatorname{rk}\left(\begin{array}{ccc}
x_{0} & s_{1} & s_{2} \\
x s_{1} & s_{2} & s_{3}
\end{array}\right)<2
$$

Now apply Lemma E.1 with $G=B_{7}, V=M^{-1} \oplus L^{-1} \oplus L^{-1}, W=\mathcal{O}_{G} \oplus \mathcal{O}_{G}$ and the map $f: V \rightarrow W$ given by the matrix:

$$
\left(\begin{array}{ccc}
x_{0} & s_{1} & s_{2} \\
x s_{1} & s_{2} & s_{3}
\end{array}\right)
$$


The quantum period Let $p_{1}, p_{2}, p_{3} \in H^{\bullet}(F ; \mathbb{Z})$ denote the first Chern classes of $L, M$ and $N$, respectively; these classes form a basis for $H^{2}(F ; \mathbb{Z})$. Write $\tau \in H^{2}(F ; \mathbb{Q})$ as $\tau=\tau_{1} p_{1}+\tau_{2} p_{2}+\tau_{3} p_{3}$ and identify the group ring $\mathbb{Q}\left[H_{2}(F ; \mathbb{Z})\right]$ with the polynomial ring $\mathbb{Q}\left[Q_{1}, Q_{2}, Q_{3}\right]$ via the $\mathbb{Q}$-linear map that sends the element $Q^{\beta} \in \mathbb{Q}\left[H_{2}(F ; \mathbb{Z})\right]$ to $Q_{1}^{\left\langle\beta, p_{1}\right\rangle} Q_{2}^{\left\langle\beta, p_{2}\right\rangle} Q_{3}^{\left\langle\beta, p_{3}\right\rangle}$. We have

$$
\begin{array}{r}
I_{F}(\tau)=e^{\tau / z} \sum_{l, m, n \geq 0} \frac{Q_{1}^{l} Q_{2}^{m} Q_{3}^{n} e^{l \tau_{1}} e^{m \tau_{2}} e^{n \tau_{3}}}{\prod_{k=1}^{l}\left(p_{1}+k z\right)^{3} \prod_{k=1}^{m}\left(p_{2}+k z\right)} \frac{\prod_{k=-\infty}^{0}\left(p_{2}-p_{1}+k z\right)}{\prod_{k=-\infty}^{m-l}\left(p_{2}-p_{1}+k z\right)} \\
\times \frac{\prod_{k=-\infty}^{0}\left(p_{3}-p_{2}+k z\right)}{\prod_{k=-\infty}^{n-m}\left(p_{3}-p_{1}+k z\right)^{2}} \\
\prod_{k=-\infty}^{n-l}\left(p_{3}-p_{2}+k z\left(p_{3}-p_{1}+k z\right)^{2}\right.
\end{array}
$$

and, since $I_{F}(\tau)=1+\tau z^{-1}+O\left(z^{-2}\right)$, Theorem C.1 gives

$$
J_{F}(\tau)=I_{F}(\tau) .
$$

We now proceed exactly as in the case of $\mathrm{MM}_{3-1}$, obtaining

$$
G_{X}(t)=e^{-t} \sum_{l=0}^{\infty} \sum_{n=l}^{\infty} \sum_{m=l}^{n} t^{m+n} \frac{n ! n !}{(l !)^{3} m r(m-l) !(n-m) !((n-l) !)^{2}} .
$$

Regularizing gives

$\widehat{G}_{X}(t)=1+4 t^{2}+18 t^{3}+84 t^{4}+540 t^{5}+3190 t^{6}+20160 t^{7}+130900 t^{8}+859320 t^{9}+\cdots$.

Minkowski period sequence 42

\section{The Fano manifold $\mathrm{MM}_{3-17}$}

Mori-Mukai construction A nonsingular divisor on $\mathbb{P}^{1} \times \mathbb{P}^{1} \times \mathbb{P}^{2}$ of tridegree $(1,1,1)$.

Our construction A member $X$ of $|L+M+N|$ on the toric variety $F$ with weight data

$$
\begin{array}{rrrrrrrr}
1 & 1 & 0 & 0 & 0 & 0 & 0 & L \\
0 & 0 & 1 & 1 & 0 & 0 & 0 & M \\
0 & 0 & 0 & 0 & 1 & 1 & 1 & N
\end{array}
$$

and Nef $F=\langle L, M, N\rangle$.

The two constructions coincide Obvious. 
The quantum period Corollary D.5 yields

$$
G_{X}(t)=e^{-2 t} \sum_{l=0}^{\infty} \sum_{m=0}^{\infty} \sum_{n=0}^{\infty} t^{l+m+2 n} \frac{(l+m+n) !}{(l !)^{2}(m !)^{2}(n !)^{3}}
$$

and regularizing gives

$$
\widehat{G}_{X}(t)=1+4 t^{2}+12 t^{3}+84 t^{4}+360 t^{5}+2380 t^{6}+13440 t^{7}+83860 t^{8}+512400 t^{9}+\cdots .
$$

Minkowski period sequence 39

\section{The Fano manifold $\mathrm{MM}_{3-18}$}

Mori-Mukai construction The blow-up of $\mathbb{P}^{3}$ with centre the disjoint union of a line and a conic.

Our construction A member $X$ of $|M+N|$ on the toric variety $F$ with weight data

\begin{tabular}{rrrrrrrl}
$s_{0}$ & $s_{1}$ & $x$ & $x_{2}$ & $x_{3}$ & $y_{0}$ & $y_{1}$ & \\
\cline { 1 - 3 } 1 & 1 & -1 & 0 & 0 & 0 & 0 & $L$ \\
0 & 0 & 1 & 1 & 1 & -1 & 0 & $M$ \\
0 & 0 & 0 & 0 & 0 & 1 & 1 & $N$
\end{tabular}

and Nef $F=\langle L, M, N\rangle$. The secondary fan of $F$ is the same as that of the toric variety in Section 57 (ie out ambient toric variety for $\mathrm{MM}_{3-4}$ ) and it is shown in Figure 2. We have:

- $-K_{F}=L+2 M+2 N$ ample, that is, $F$ is a Fano variety.

- $X \sim M+N$ is nef.

- $-\left(K_{F}+X\right) \sim L+M+N$ is ample.

The two constructions coincide We construct $X$, for example, as the blow-up of $\mathbb{P}_{x_{0}, x_{1}, x_{2}, x_{3}}^{3}$ along the (disjoint) union of the line $\left(x_{0}=x_{1}=0\right)$ and the conic $\left(x_{0} x_{1}+x_{2}^{2}=x_{3}=0\right)$. Thus $X$ is given in $F$ by the equation

$$
y_{0}\left(s_{0} s_{1} x^{2}+x_{2}^{2}\right)+y_{1} x_{3}=0,
$$

where the morphism $F \rightarrow \mathbb{P}^{3}$ is given (contravariantly) by

$$
\left[x_{0}, x_{1}, x_{2}, x_{3}\right] \mapsto\left[s_{0} x, s_{1} x, x_{2}, x_{3}\right] .
$$


The quantum period Corollary D.5 yields

$$
G_{X}(t)=e^{-t} \sum_{l=0}^{\infty} \sum_{m=l}^{\infty} \sum_{n=m}^{\infty} t^{l+m+n} \frac{(m+n) !}{(l !)^{2}(m-l) !(m !)^{2}(n-m) ! n !}
$$

and regularizing gives

$\widehat{G}_{X}(t)=1+4 t^{2}+18 t^{3}+60 t^{4}+480 t^{5}+2470 t^{6}+14280 t^{7}+94780 t^{8}+564480 t^{9}+\cdots$.

Minkowski period sequence 41

\section{The Fano manifold $\mathrm{MM}_{3-19}$}

Mori-Mukai construction The blow-up of a quadric 3-fold $Q \subset \mathbb{P}^{4}$ with centre two points $P_{1}$ and $P_{2}$ on it which are not collinear.

Our construction A member $X$ of $|2 M|$ in the rank- 2 toric variety $F$ with weight data

\begin{tabular}{rrrrrrr}
$s_{0}$ & $s_{1}$ & $s_{2}$ & $x$ & $x_{3}$ & $x_{4}$ & \\
\cline { 1 - 5 } & 1 & 1 & -1 & 0 & 0 & $L$ \\
0 & 0 & 0 & 1 & 1 & 1 & $M$
\end{tabular}

and Nef $F=\langle L, M\rangle$. We have:

- $-K_{F}=2 L+3 M$ is ample, that is, $F$ is a Fano variety.

- $\quad X \sim 2 M$ is nef.

- $-\left(K_{F}+X\right) \sim 2 L+M$ is ample.

The two constructions coincide $F$ is manifestly the blow-up of $\mathbb{P}_{x_{0}, x_{1}, x_{2}, x_{3}, x_{4}}^{4}$ along the line $\left(x_{0}=x_{1}=x_{2}=0\right)$, and $X$ is the strict transform of a general quadric.

The quantum period Corollary D.5 yields

and regularizing gives

$$
G_{X}(t)=e^{-2 t} \sum_{l=0}^{\infty} \sum_{m=l}^{\infty} t^{2 l+m} \frac{(2 m) !}{(l !)^{3}(m-l) !(m !)^{2}}
$$

$\widehat{G}_{X}(t)=1+2 t^{2}+12 t^{3}+54 t^{4}+240 t^{5}+1280 t^{6}+7560 t^{7}+42070 t^{8}+235200 t^{9}+\cdots$.

Minkowski period sequence 18 


\section{The Fano manifold $M_{3-20}$}

Mori-Mukai construction The blow-up of a quadric 3 -fold $Q \subset \mathbb{P}^{4}$ with centre two disjoint lines on it.

Our construction A member $X$ of $|L+M|$ in the toric variety $F$ with weight data

\begin{tabular}{rrrrrrrr}
$s_{0}$ & $s_{1}$ & $t_{2}$ & $t_{3}$ & $u_{4}$ & $x$ & $y$ & \\
\cline { 1 - 6 } 1 & 1 & 0 & 0 & 1 & -1 & 0 & $L$ \\
0 & 0 & 1 & 1 & 1 & 0 & -1 & $M$ \\
0 & 0 & 0 & 0 & -1 & 1 & 1 & $N$
\end{tabular}

and Nef $F=\langle L, M, N\rangle$. The secondary fan of $F$ is the same as that for $F$ in $\mathrm{MM}_{3-16}$; it is shown schematically in Figure 7.

We have:

- $-K_{F}=2 L+2 M+N$ is ample, that is, $F$ is a Fano variety.

- $\quad X \sim L+M$ is nef.

- $-\left(K_{F}+X\right) \sim L+M+N$ is ample.

The two constructions coincide We blow up the disjoint union of the two lines $\left(x_{2}=x_{3}=x_{4}=0\right)$ and $\left(x_{0}=x_{1}=x_{4}=0\right)$ in $\mathbb{P}_{x_{0}, x_{1}, x_{2}, x_{3}, x_{4}}^{4}$ and take $X$ to be the proper transform of the quadric $x_{0} x_{3}+x_{1} x_{2}+x_{4}^{2}=0$ constructed to contain the two lines. The morphism $F \rightarrow \mathbb{P}^{4}$ is given (contravariantly) by

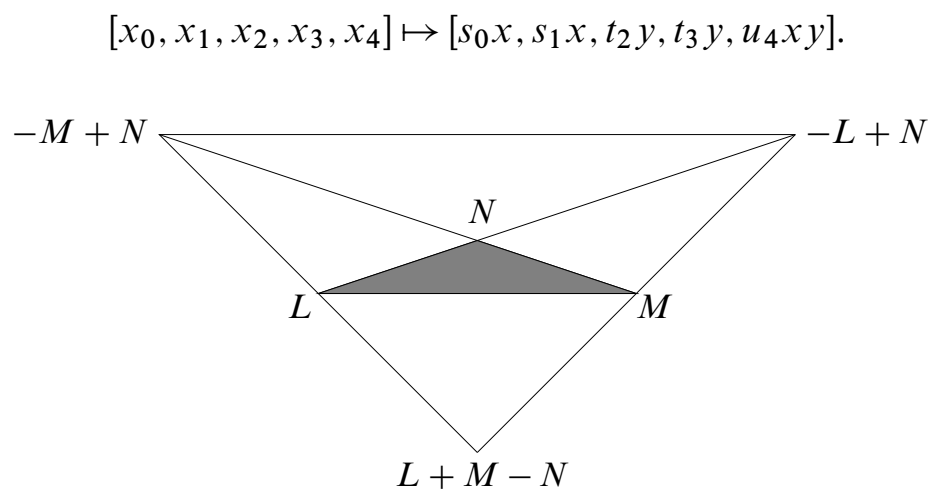

Figure 7: The secondary fan for $F$ in $\mathrm{MM}_{3-20}$. 
The quantum period Corollary D.5 yields

$$
G_{X}(t)=\sum_{l=0}^{\infty} \sum_{m=0}^{\infty} \sum_{n=\max (l, m)}^{l+m} t^{l+m+n} \frac{(l+m) !}{(l !)^{2}(m !)^{2}(l+m-n) !(n-l) !(n-m) !}
$$

and regularizing gives

$\widehat{G}_{X}(t)=1+4 t^{2}+12 t^{3}+60 t^{4}+360 t^{5}+1660 t^{6}+10920 t^{7}+57820 t^{8}+361200 t^{9}+\cdots$.

Minkowski period sequence 38

\section{The Fano manifold $M_{3-21}$}

Mori-Mukai construction The blow-up of $\mathbb{P}^{1} \times \mathbb{P}^{2}$ with centre a curve of bidegree $(2,1)$.

Our construction A member $X$ of $|M+N|$ on the toric variety $F$ with weight data

\begin{tabular}{cccccccc}
$x_{0}$ & $x_{1}$ & $y_{0}$ & $y_{1}$ & $y_{2}$ & $s$ & $t$ & \\
\cline { 1 - 6 } 1 & 1 & 0 & 0 & 0 & 0 & -1 & $L$ \\
0 & 0 & 1 & 1 & 1 & 0 & -1 & $M$ \\
0 & 0 & 0 & 0 & 0 & 1 & 1 & $N$
\end{tabular}

and Nef $F=\langle L, M, N\rangle$. The secondary fan of $F$ is the same as of the toric variety in Section 55 (ie our ambient toric variety for $\mathrm{MM}_{3-2}$ ) and is shown in Figure 1. We have:

- $-K_{F}=L+2 M+2 N$ is ample, that is, $F$ is a Fano variety.

- $X \sim M+N$ is nef.

- $-\left(K_{F}+X\right) \sim L+M+N$ is ample.

The two constructions coincide A complete intersection of type $(0,1) \cap(1,2)$ on $\mathbb{P}^{1} \times \mathbb{P}^{2}$ is a curve of bidegree (2,1). Apply Lemma E. 1 with $G=\mathbb{P}_{x_{0}, x_{1}}^{1} \times \mathbb{P}_{y_{0}, y_{1}, y_{2}}^{2}$, $V=\mathcal{O}_{\mathbb{P}^{1} \times \mathbb{P}^{2}} \oplus \mathcal{O}_{\mathbb{P}^{1} \times \mathbb{P}^{2}}(-1,-1), W=\mathcal{O}_{\mathbb{P}^{1} \times \mathbb{P}^{2}}(0,1)$ and $f: V \rightarrow W$ given by the matrix $\left(y_{0} x_{0} q_{0}+x_{1} q_{1}\right)$, where $q_{0}$ and $q_{1}$ are homogeneous quadratic polynomials in $y_{0}, y_{1}$ and $y_{2}$.

The quantum period Corollary D.5 yields

$$
G_{X}(t)=e^{-t} \sum_{l=0}^{\infty} \sum_{m=0}^{\infty} \sum_{n=l+m}^{\infty} t^{l+m+n} \frac{(m+n) !}{(l !)^{2}(m !)^{3} n !(n-l-m) !}
$$

and regularizing gives

$\widehat{G}_{X}(t)=1+6 t^{2}+6 t^{3}+114 t^{4}+240 t^{5}+3030 t^{6}+9660 t^{7}+95970 t^{8}+394800 t^{9}+\cdots$. 
Minkowski period sequence 49

\section{The Fano manifold $\mathrm{MM}_{3-22}$}

Mori-Mukai construction The blow-up of $\mathbb{P}^{1} \times \mathbb{P}^{2}$ with centre a conic in $t \times \mathbb{P}^{2}$ $\left(t \in \mathbb{P}^{1}\right)$.

Our construction A member $X$ of $|N|$ on the toric variety $F$ with weight data

\begin{tabular}{rrrrrrrl}
$x_{0}$ & $x_{1}$ & $y_{0}$ & $y_{1}$ & $y_{2}$ & $s$ & $t$ & \\
\cline { 1 - 5 } 1 & 1 & 0 & 0 & 0 & -1 & 0 & $L$ \\
0 & 0 & 1 & 1 & 1 & 0 & -2 & $M$ \\
0 & 0 & 0 & 0 & 0 & 1 & 1 & $N$
\end{tabular}

and Nef $F=\langle L, M, N\rangle$. The secondary fan of $F$ is similar to that of our ambient toric variety for $\mathrm{MM}_{3-10}$; cf Figure 4. We have:

- $-K_{F}=L+M+2 N$ is ample, that is, $F$ is a Fano variety.

- $\quad X \sim N$ is nef.

- $-\left(K_{F}+X\right) \sim L+M+N$ is ample.

The two constructions coincide Apply Lemma E.1 with $G=\mathbb{P}_{x_{0}, x_{1}}^{1} \times \mathbb{P}_{y_{0}, y_{1}, y_{2}}^{2}$, $V=\mathcal{O}_{\mathbb{P}^{1} \times \mathbb{P}^{2}}(-1,0) \oplus \mathcal{O}_{\mathbb{P}^{1} \times \mathbb{P}^{2}}(0,-2), W=\mathcal{O}_{\mathbb{P}^{1} \times \mathbb{P}^{2}}$ and $f: V \rightarrow W$ given by the matrix $\left(x_{0}-t x_{1} \quad y_{0} y_{2}-y_{1}^{2}\right)$.

The quantum period Corollary D.5 yields

$$
G_{X}(t)=e^{-t} \sum_{l=0}^{\infty} \sum_{m=0}^{\infty} \sum_{n=\max (l, 2 m)}^{\infty} t^{l+m+n} \frac{n !}{(l !)^{2}(m !)^{3}(n-l) !(n-2 m) !}
$$

and regularizing gives

$\widehat{G}_{X}(t)=1+2 t^{2}+6 t^{3}+54 t^{4}+180 t^{5}+830 t^{6}+4620 t^{7}+26950 t^{8}+140280 t^{9}+\cdots$.

Minkowski period sequence 13

\section{The Fano manifold $\mathrm{MM}_{3-23}$}

Mori-Mukai construction The blow-up of $B_{7}\left(\mathrm{MM}_{2-35}\right)$ with centre a conic passing through the centre of the blow-up $B_{7} \rightarrow \mathbb{P}^{3}$. 
Our construction A member $X$ of $|L+N|$ in the toric variety $F$ with weight data

\begin{tabular}{rrrrrrrr}
$s_{1}$ & $s_{2}$ & $s_{3}$ & $x$ & $x_{0}$ & $u$ & $v$ & \\
\cline { 1 - 3 } & 1 & 1 & -1 & 0 & 0 & 0 & $L$ \\
0 & 0 & 0 & 1 & 1 & -1 & 0 & $M$ \\
0 & 0 & 0 & 0 & 0 & 1 & 1 & $N$
\end{tabular}

and Nef $F=\langle L, M, N\rangle$. The secondary fan for $F$ is the same as that of the toric variety in Section 57 (ie our ambient toric variety for $\mathrm{MM}_{3-4}$ ) and is shown in Figure 2. We have:

- $-K_{F}=2 L+M+2 N$ is ample, that is, $F$ is a Fano variety.

- $X \sim L+N$ is nef.

- $-\left(K_{F}+X\right) \sim L+M+N$ is ample.

The two constructions coincide Consider the conic $\Gamma$ given by $\left(x_{3}=x_{0} x_{1}+x_{2}^{2}=0\right)$ in $\mathbb{P}_{x_{0}, \ldots, x_{3}}^{3}$ and note that $P=[1: 0: 0: 0]$ lies on $\Gamma$. Recall that $B_{7}$ is the toric variety with weight data

\begin{tabular}{rrrrrr}
$s_{1}$ & $s_{2}$ & $s_{3}$ & $x$ & $x_{0}$ & \\
\cline { 1 - 4 } & 1 & 1 & -1 & 0 & $L$ \\
0 & 0 & 0 & 1 & 1 & $M$
\end{tabular}

and Nef $B_{7}=\langle L, M\rangle$, and that the blow-up morphism $B_{7} \rightarrow \mathbb{P}^{3}$ is given (contravariantly) by $\left[x_{0}, x_{1}, x_{2}, x_{3}\right] \mapsto\left[x_{0}, s_{1} x, s_{2} x, s_{3} x\right]$. The proper transform of the curve $\Gamma$ is the curve $\Gamma^{\prime}$ defined by the equations

$$
s_{3}=x_{0} s_{1}+x s_{2}^{2}=0 .
$$

Now apply Lemma E.1 with $G=B_{7}, V=M^{-1} \oplus \mathcal{O}_{G}, W=L$ and the map $f: V \rightarrow W$ given by the matrix $\left(x_{0} s_{1}+x s_{2}^{2} s_{3}\right)$.

The quantum period Corollary D.5 yields

$$
G_{X}(t)=e^{-t} \sum_{l=0}^{\infty} \sum_{m=l}^{\infty} \sum_{n=m}^{\infty} t^{l+m+n} \frac{(l+n) !}{(l !)^{3}(m-l) ! m !(n-m) ! n !}
$$

and regularizing gives

$\widehat{G}_{X}(t)=1+2 t^{2}+12 t^{3}+30 t^{4}+180 t^{5}+920 t^{6}+4200 t^{7}+22750 t^{8}+121800 t^{9}+\cdots$. 


\section{The Fano manifold $\mathbf{M M}_{3-24}$}

Mori-Mukai construction The fibre product $W \times_{\mathbb{P}^{2}} \mathbb{F}_{1}$, where $W \rightarrow \mathbb{P}^{2}$ is a $\mathbb{P}^{1}$ bundle and $p: \mathbb{F}_{1} \rightarrow \mathbb{P}^{2}$ is the blow-up. Here $W\left(\mathrm{MM}_{2-32}\right)$ is a divisor of bidegree $(1,1)$ on $\mathbb{P}^{2} \times \mathbb{P}^{2}$.

Our construction A member $X$ of $|M+N|$ on the toric variety $\mathbb{F}_{1} \times \mathbb{P}^{2}$, where $M$ is the line bundle $p^{\star} \mathcal{O}(1)$ on $\mathbb{F}_{1}$ and $N=\mathcal{O}(1)$. In other words, $X$ is a member of $|M+N|$ on the toric variety $F$ with weight data

\begin{tabular}{cccccccc}
$s_{0}$ & $s_{1}$ & $x$ & $x_{2}$ & $y_{0}$ & $y_{1}$ & $y_{2}$ & \\
\cline { 1 - 6 } 1 & 1 & -1 & 0 & 0 & 0 & 0 & $L$ \\
0 & 0 & 1 & 1 & 0 & 0 & 0 & $M$ \\
0 & 0 & 0 & 0 & 1 & 1 & 1 & $N$
\end{tabular}

and Nef $F=\langle L, M, N\rangle$. We have:

- $-K_{F}=L+2 M+3 N$ is ample, that is, $F$ is a Fano variety.

- $\quad X \sim M+N$ is nef.

- $-\left(K_{F}+X\right) \sim L+M+2 N$ is ample.

The two constructions coincide First we show that $X$ is the blow-up of $\mathbb{P}^{1} \times \mathbb{P}^{2}$ along a curve of bidegree $(1,1)$. Note that $X$ is cut out of $\mathbb{P}_{x_{0}, x_{1}, x_{2}}^{2} \times \mathbb{P}_{y_{0}, y_{1}, y_{2}}^{2} \times \mathbb{P}_{s_{0}, s_{1}}^{1}$ by the equations

$$
\left\{\begin{aligned}
y_{0} x_{0}+y_{1} x_{1}+y_{2} x_{2} & =0 \\
s_{0} x_{0}+s_{1} x_{1} & =0
\end{aligned}\right.
$$

The first equation here cuts $W$ out of $\mathbb{P}_{x_{0}, x_{1}, x_{2}}^{2} \times \mathbb{P}_{y_{0}, y_{1}, y_{2}}^{2}$; the second equation cuts $\mathbb{F}_{1}$ out of $\mathbb{P}_{x_{0}, x_{1}, x_{2}}^{2} \times \mathbb{P}_{s_{0}, s_{1}}^{1}$, as it is the equation defining the blow-up of $\mathbb{P}^{2}$ at the point $[0: 0: 1]$. We now exhibit $X$ as the blow-up of a curve in $\mathbb{P}_{y_{0}, y_{1}, y_{2}}^{2} \times \mathbb{P}_{s_{0}, s_{1}}^{1}$. The projection to $\mathbb{P}_{y_{0}, y_{1}, y_{2}}^{2} \times \mathbb{P}_{s_{0}, s_{1}}^{1}$ is an isomorphism away from the locus where the matrix

$$
\left(\begin{array}{lll}
y_{0} & y_{1} & y_{2} \\
s_{0} & s_{1} & 0
\end{array}\right)
$$

drops rank. This locus is

$$
\left\{\begin{aligned}
y_{2} & =0 \\
y_{0} s_{1}-y_{1} s_{0} & =0
\end{aligned}\right.
$$

ie a curve in of bidegree $(1,1)$, as claimed. We can further simplify things by writing $X$ as a hypersurface in $\mathbb{F}_{1} \times \mathbb{P}^{2}$ : the two equations defining $X$ (given above) reduce to the single equation

$$
s_{0} x y_{0}+s_{1} x y_{1}+x_{2} y_{2}=0
$$

in $\mathbb{F}^{1} \times \mathbb{P}^{2}$. 
The quantum period Corollary D.5 yields

$$
G_{X}(t)=e^{-t} \sum_{l=0}^{\infty} \sum_{m=l}^{\infty} \sum_{n=0}^{\infty} t^{l+m+2 n} \frac{(m+n) !}{(l !)^{2}(m-l) ! m !(n !)^{3}}
$$

and regularizing gives

$\widehat{G}_{X}(t)=1+4 t^{2}+6 t^{3}+60 t^{4}+180 t^{5}+1210 t^{6}+5460 t^{7}+30940 t^{8}+165480 t^{9}+\cdots$.

Minkowski period sequence 31

\section{The Fano manifold $M_{3-25}$}

Mori-Mukai construction The blow-up of $\mathbb{P}^{3}$ with centre two disjoint lines; equivalently, ${ }^{17} \mathbb{P}(\mathcal{O}(1,0) \oplus \mathcal{O}(0,1))$ over $\mathbb{P}^{1} \times \mathbb{P}^{1}$.

Our construction The toric variety $X$ with weight data

\begin{tabular}{rrrrrrr}
$s_{0}$ & $s_{1}$ & $t_{2}$ & $t_{3}$ & $x$ & $y$ & \\
\cline { 1 - 5 } 1 & 1 & 0 & 0 & -1 & 0 & $L$ \\
0 & 0 & 1 & 1 & 0 & -1 & $M$ \\
0 & 0 & 0 & 0 & 1 & 1 & $N$
\end{tabular}

and $\operatorname{Nef} X=\langle L, M, N\rangle$.

The two constructions coincide The morphism $X \rightarrow \mathbb{P}^{3}$ that sends (contravariantly) the homogeneous co-ordinate functions $\left[x_{0}, x_{1}, x_{2}, x_{3}\right]$ to $\left[s_{0} x, s_{1} x, t_{2} y, t_{3} y\right]$ manifestly blows up the union of the line $\left(x_{0}=x_{1}=0\right)$ and the line $\left(x_{2}=x_{3}=0\right)$. These lines are disjoint.

The quantum period Corollary C.2 yields

$$
G_{X}(t)=\sum_{l=0}^{\infty} \sum_{m=0}^{\infty} \sum_{n=\max (l, m)}^{\infty} \frac{t^{l+m+2 n}}{(l !)^{2}(m !)^{2}(n-l) !(n-m) !}
$$

and regularizing gives

$\widehat{G}_{X}(t)=1+2 t^{2}+12 t^{3}+30 t^{4}+120 t^{5}+920 t^{6}+3360 t^{7}+16030 t^{8}+99120 t^{9}+\cdots$.

Minkowski period sequence 16

${ }^{17}$ Note that Mori and Mukai use different weight conventions for projective bundles than we do. 


\section{The Fano manifold $M_{3-26}$}

Mori-Mukai construction The blow-up of $\mathbb{P}^{3}$ with centre a disjoint union of a point and a line.

Our construction The toric variety $X$ with weight data

\begin{tabular}{rrrrrrr}
$s_{0}$ & $s_{1}$ & $t_{2}$ & $u_{3}$ & $x$ & $y$ & \\
\cline { 1 - 5 } 1 & 1 & 0 & 1 & -1 & 0 & $L$ \\
0 & 0 & 1 & 1 & 0 & -1 & $M$ \\
0 & 0 & 0 & -1 & 1 & 1 & $N$
\end{tabular}

and Nef $X=\langle L, M, N\rangle$. The secondary fan of $X$ is the same as that of the toric variety in $\mathrm{MM}_{3-20}$ and it is shown in Figure 7.

The two constructions coincide The morphism to $\mathbb{P}^{3}$ is given by the complete linear system $|N|$ on $X$; it sends (contravariantly) the homogeneous co-ordinates $\left[x_{0}, x_{1}, x_{2}, x_{3}\right]$ to $\left[s_{0} x, s_{1} x, t_{2} y, u_{3} x y\right]$. The divisor $(x=0) \subset X$ contracts to the point $[0: 0: 1: 0] \in \mathbb{P}^{3}$ and the divisor $(y=0) \subset X$ contracts to the line $\left(x_{2}=x_{3}=0\right) \subset \mathbb{P}^{3}$.

The quantum period Corollary C.2 yields

$$
G_{X}(t)=\sum_{l=0}^{\infty} \sum_{m=0}^{\infty} \sum_{n=\max (l, m)}^{l+m} \frac{t^{2 l+m+n}}{(l !)^{2} m !(l+m-n) !(n-l) !(n-m) !}
$$

and regularizing gives

$\widehat{G}_{X}(t)=1+2 t^{2}+6 t^{3}+30 t^{4}+120 t^{5}+470 t^{6}+2520 t^{7}+10990 t^{8}+57120 t^{9}+\cdots$.

Minkowski period sequence 12

\section{The Fano manifold $\mathrm{MM}_{3-27}$}

Mori-Mukai construction $\mathbb{P}^{1} \times \mathbb{P}^{1} \times \mathbb{P}^{1}$.

Our construction The toric variety $X$ with weight data

$\begin{array}{lllllll}1 & 1 & 0 & 0 & 0 & 0 & L \\ 0 & 0 & 1 & 1 & 0 & 0 & M \\ 0 & 0 & 0 & 0 & 1 & 1 & N\end{array}$

and Nef $X=\langle L, M, N\rangle$. 
The two constructions coincide Obvious.

The quantum period Corollary C.2 yields

and regularizing gives

$$
G_{X}(t)=\sum_{l=0}^{\infty} \sum_{m=0}^{\infty} \sum_{n=0}^{\infty} \frac{t^{2 l+2 m+2 n}}{(l !)^{2}(m !)^{2}(n !)^{2}}
$$

$$
\widehat{G}_{X}(t)=1+6 t^{2}+90 t^{4}+1860 t^{6}+44730 t^{8}+1172556 t^{10}+\cdots .
$$

Minkowski period sequence 45

\section{The Fano manifold $\mathrm{MM}_{3-28}$}

Mori-Mukai construction $\mathbb{P}^{1} \times \mathbb{F}_{1}$.

Our construction The toric variety $X$ with weight data

$\begin{array}{rrrrrrc}1 & 1 & 0 & 0 & 0 & 0 & L \\ 0 & 0 & 1 & 1 & -1 & 0 & M \\ 0 & 0 & 0 & 0 & 1 & 1 & N\end{array}$

and Nef $X=\langle L, M, N\rangle$.

The two constructions coincide Obvious.

The quantum period Corollary C.2 yields

and regularizing gives

$$
G_{X}(t)=\sum_{l=0}^{\infty} \sum_{m=0}^{\infty} \sum_{n=m}^{\infty} \frac{t^{2 l+m+2 n}}{(l !)^{2}(m !)^{2}(n-m) ! n !}
$$

$\widehat{G}_{X}(t)=1+4 t^{2}+6 t^{3}+36 t^{4}+180 t^{5}+490 t^{6}+4200 t^{7}+11620 t^{8}+89880 t^{9}+\cdots$.

Minkowski period sequence 28

\section{The Fano manifold $\mathrm{MM}_{3-29}$}

Mori-Mukai construction The blow-up of $B_{7}\left(\mathrm{MM}_{2-35}\right)$ with centre a line on the exceptional divisor $D \cong \mathbb{P}^{2}$ of the blow-up $B_{7} \rightarrow \mathbb{P}^{3}$. 


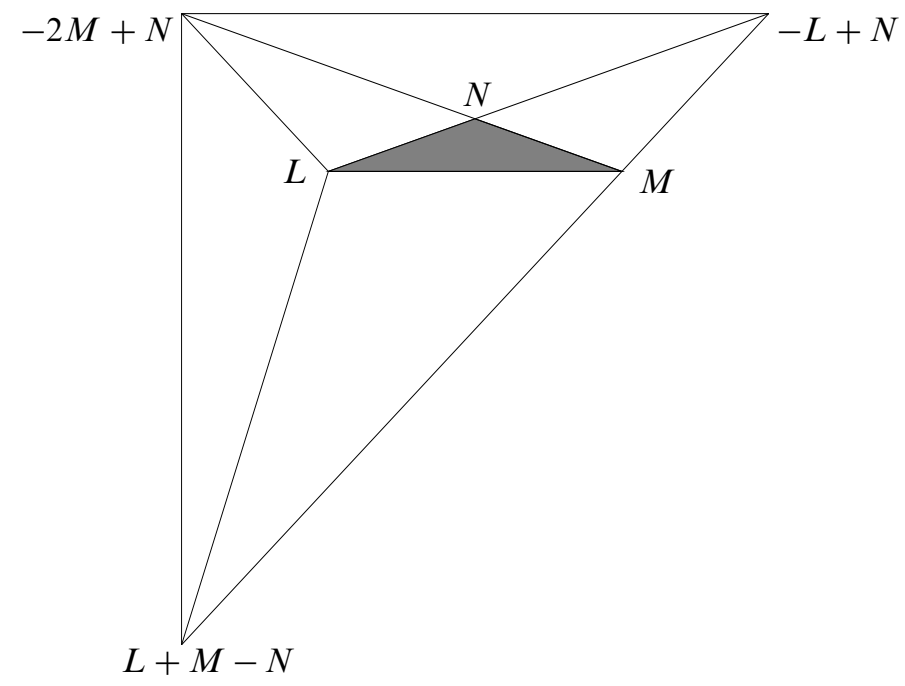

Figure 8: The secondary fan for $X$ in $\mathrm{MM}_{3-29}$.

Our construction The toric variety $X$ with weight data

\begin{tabular}{rrrrrrr}
$x_{0}$ & $s_{1}$ & $s_{2}$ & $t_{3}$ & $x$ & $y$ & \\
\cline { 1 - 3 } 1 & 0 & 0 & -1 & 0 & 1 & $L$ \\
0 & 1 & 1 & 0 & -2 & 1 & $M$ \\
0 & 0 & 0 & 1 & 1 & -1 & $N$
\end{tabular}

and Nef $X=\langle L, M, N\rangle$. The secondary fan of $X$ is shown schematically in Figure 8 .

The two constructions coincide The morphism $X \rightarrow \mathbb{P}^{3}$ sends (contravariantly) the homogeneous co-ordinate functions $\left[x_{0}, x_{1}, x_{2}, x_{3}\right]$ to $\left[x_{0}, s_{1} x y, s_{2} x y, t_{3} x y^{2}\right]$.

The quantum period Corollary C.2 yields

$$
G_{X}(t)=\sum_{l=0}^{\infty} \sum_{m=0}^{\infty} \sum_{n=\max (l, 2 m)}^{l+m} \frac{t^{l+m+n}}{l !(m !)^{2}(n-l) !(n-2 m) !(l+m-n) !}
$$

and regularizing gives

$$
\widehat{G}_{X}(t)=1+2 t^{2}+30 t^{4}+60 t^{5}+380 t^{6}+840 t^{7}+5950 t^{8}+22680 t^{9}+\cdots
$$

\section{Minkowski period sequence 8}




\section{The Fano manifold $\mathrm{MM}_{3-30}$}

Mori-Mukai construction The blow-up of $B_{7}\left(\mathrm{MM}_{2-35}\right)$ with centre the strict transform of a line passing through the centre of the blow-up $B_{7} \rightarrow \mathbb{P}^{3}$.

Our construction The toric variety $X$ with weight data

\begin{tabular}{rrrrrrr}
$t_{0}$ & $t_{1}$ & $x$ & $s_{2}$ & $y$ & $x_{3}$ & \\
\cline { 1 - 5 } 1 & 1 & -1 & 0 & 0 & 0 & $L$ \\
0 & 0 & 1 & 1 & -1 & 0 & $M$ \\
0 & 0 & 0 & 0 & 1 & 1 & $N$
\end{tabular}

The two constructions coincide The morphism $X \rightarrow \mathbb{P}^{3}$ sends (contravariantly) the homogeneous co-ordinate functions $\left[x_{0}, x_{1}, x_{2}, x_{3}\right]$ to $\left[t_{0} x y, t_{1} x y, s_{2} y, x_{3}\right]$.

The quantum period Corollary C.2 yields

and regularizing gives

$$
G_{X}(t)=\sum_{l=0}^{\infty} \sum_{m=l}^{\infty} \sum_{n=m}^{\infty} \frac{t^{l+m+2 n}}{(l !)^{2}(m-l) ! m !(n-m) ! n !}
$$

$\widehat{G}_{X}(t)=1+2 t^{2}+6 t^{3}+30 t^{4}+60 t^{5}+470 t^{6}+1680 t^{7}+7630 t^{8}+34440 t^{9}+\cdots$.

Minkowski period sequence 11

\section{The Fano manifold $M_{3-31}$}

Mori-Mukai construction The blow-up of the cone over a nonsingular quadric surface in $\mathbb{P}^{3}$ with centre the vertex; equivalently, the $\mathbb{P}^{1}$-bundle $\mathbb{P}(\mathcal{O} \oplus \mathcal{O}(1,1))$ over $\mathbb{P}^{1} \times \mathbb{P}^{1}$.

Our construction The toric variety $X$ with weight data

\begin{tabular}{rrrrrrr}
$s_{0}$ & $s_{1}$ & $t_{0}$ & $t_{1}$ & $x$ & $y$ & \\
\cline { 1 - 5 } 1 & 1 & 0 & 0 & -1 & 0 & $L$ \\
0 & 0 & 1 & 1 & -1 & 0 & $M$ \\
0 & 0 & 0 & 0 & 1 & 1 & $N$
\end{tabular}

and Nef $X=\langle L, M, N\rangle$.

The two constructions coincide Obvious. 
The quantum period Corollary C.2 yields

and regularizing gives

$$
G_{X}(t)=\sum_{l=0}^{\infty} \sum_{m=0}^{\infty} \sum_{n=l+m}^{\infty} \frac{t^{l+m+2 n}}{(l !)^{2}(m !)^{2}(n-l-m) ! n !}
$$

$\widehat{G}_{X}(t)=1+2 t^{2}+12 t^{3}+6 t^{4}+120 t^{5}+560 t^{6}+840 t^{7}+10150 t^{8}+38640 t^{9}+\cdots$.

Minkowski period sequence 14

\section{The Fano manifold $\mathrm{MM}_{4-1}$}

Mori-Mukai construction A divisor of multidegree $(1,1,1,1)$ in $\mathbb{P}^{1} \times \mathbb{P}^{1} \times \mathbb{P}^{1} \times \mathbb{P}^{1}$.

Our construction A member $X$ of $|A+B+C+D|$ in the toric variety $F$ with weight data

$$
\begin{array}{lllllllll}
1 & 1 & 0 & 0 & 0 & 0 & 0 & 0 & A \\
0 & 0 & 1 & 1 & 0 & 0 & 0 & 0 & B \\
0 & 0 & 0 & 0 & 1 & 1 & 0 & 0 & C \\
0 & 0 & 0 & 0 & 0 & 0 & 1 & 1 & D
\end{array}
$$

and Nef $X=\langle A, B, C, D\rangle$.

The two constructions coincide Obvious.

The quantum period Corollary D.5 yields

$$
G_{X}(t)=e^{-4 t} \sum_{a=0}^{\infty} \sum_{b=0}^{\infty} \sum_{c=0}^{\infty} \sum_{d=0}^{\infty} t^{a+b+c+d} \frac{(a+b+c+d) !}{(a !)^{2}(b !)^{2}(c !)^{2}(d !)^{2}}
$$

and regularizing gives

$$
\begin{aligned}
\widehat{G}_{X}(t)=1+12 t^{2}+48 t^{3} & +540 t^{4}+4320 t^{5} \\
& +42240 t^{6}+403200 t^{7}+4038300 t^{8}+40958400 t^{9}+\cdots .
\end{aligned}
$$

Minkowski period sequence 111

\section{The Fano manifold $\mathrm{MM}_{4-2}$}

Mori-Mukai construction The blow-up of $\mathbb{P}^{1} \times \mathbb{P}^{1} \times \mathbb{P}^{1}$ with centre a curve of tridegree $(1,1,3) .^{18}$

\footnotetext{
${ }^{18}$ Mori and Mukai initially missed this variety [50; 53]. We put it where it belongs in their scheme.
} 
Our construction A member $X$ of $|B+C+D|$ in the toric variety $F$ with weight data

\begin{tabular}{rrrrrrrrr}
$x_{0}$ & $x_{1}$ & $y_{0}$ & $y_{1}$ & $z_{0}$ & $z_{1}$ & $u$ & $v$ & \\
\hline 1 & 1 & 0 & 0 & 0 & 0 & -1 & 0 & $A$ \\
0 & 0 & 1 & 1 & 0 & 0 & -1 & 0 & $B$ \\
0 & 0 & 0 & 0 & 1 & 1 & 0 & 0 & $C$ \\
0 & 0 & 0 & 0 & 0 & 0 & 1 & 1 & $D$
\end{tabular}

and Nef $F=\langle A, B, C, D\rangle$. We have:

- $-K_{F}=A+B+2 C+2 D$ is ample, that is, $F$ is a Fano variety.

- $X \sim B+C+D$ is nef.

- $-\left(K_{F}+X\right) \sim A+C+D$ is nef and big but not ample.

The two constructions coincide The curve is a complete intersection of type

$$
(1,2,1) \cap(0,1,1)
$$

in $\mathbb{P}^{1} \times \mathbb{P}^{1} \times \mathbb{P}^{1}$, so $X$ is constructed by applying Lemma E. 1 with

$$
\begin{aligned}
G & =\mathbb{P}^{1} \times \mathbb{P}^{1} \times \mathbb{P}^{1}, \\
V & =\mathcal{O}_{\mathbb{P}^{1} \times \mathbb{P}^{1} \times \mathbb{P}^{1}}(-1,-1,0) \oplus \mathcal{O}_{\mathbb{P}^{1} \times \mathbb{P}^{1} \times \mathbb{P}^{1},} \\
W & =\mathcal{O}_{\mathbb{P}^{1} \times \mathbb{P}^{1} \times \mathbb{P}^{1}}(0,1,1),
\end{aligned}
$$

and $f: V \rightarrow W$ given by the matrix $(A B)$, where

$A \in \Gamma\left(\mathbb{P}^{1} \times \mathbb{P}^{1} \times \mathbb{P}^{1} ; \mathcal{O}_{\mathbb{P}^{1} \times \mathbb{P}^{1} \times \mathbb{P}^{1}}(1,2,1)\right), \quad B \in \Gamma\left(\mathbb{P}^{1} \times \mathbb{P}^{1} \times \mathbb{P}^{1} ; \mathcal{O}_{\mathbb{P}^{1} \times \mathbb{P}^{1} \times \mathbb{P}^{1}}(0,1,1)\right)$, are the sections that define the centre of the blow-up.

The quantum period Let $p_{1}, p_{2}, p_{3}, p_{4} \in H^{\bullet}(F ; \mathbb{Z})$ denote the first Chern classes of $A, B, C$ and $D$, respectively; these classes form a basis for $H^{2}(F ; \mathbb{Z})$. Write $\tau \in H^{2}(F ; \mathbb{Q})$ as $\tau=\tau_{1} p_{1}+\tau_{2} p_{2}+\tau_{3} p_{3}+\tau_{4} p_{4}$ and identify the group ring $\mathbb{Q}\left[H_{2}(F ; \mathbb{Z})\right]$ with the polynomial ring $\mathbb{Q}\left[Q_{1}, Q_{2}, Q_{3}, Q_{4}\right]$ via the $\mathbb{Q}$-linear map that sends the element $Q^{\beta} \in \mathbb{Q}\left[H_{2}(F ; \mathbb{Z})\right]$ to $Q_{1}^{\left\langle\beta, p_{1}\right\rangle} Q_{2}^{\left\langle\beta, p_{2}\right\rangle} Q_{3}^{\left\langle\beta, p_{3}\right\rangle} Q_{4}^{\left\langle\beta, p_{4}\right\rangle}$. Theorem C.1 gives

$J_{F}(\tau)$

$$
\begin{array}{r}
=e^{\tau / z} \sum_{a, b, c, d \geq 0} \frac{Q_{1}^{a} Q_{2}^{b} Q_{3}^{c} Q_{4}^{d} e^{a \tau_{1}} e^{b \tau_{2}} e^{c \tau_{3}} e^{d \tau_{4}}}{\prod_{k=1}^{a}\left(p_{1}+k z\right)^{2} \prod_{k=1}^{b}\left(p_{2}+k z\right)^{2} \prod_{k=1}^{c}\left(p_{3}+k z\right)^{2} \prod_{k=1}^{d}\left(p_{4}+k z\right)} \\
\times \frac{\prod_{k=-\infty}^{0}\left(p_{4}-p_{1}-p_{2}+k z\right)}{\prod_{k=-\infty}^{d-a-b}\left(p_{4}-p_{1}-p_{2}+k z\right)}
\end{array}
$$


and hence

$I_{\boldsymbol{e}, E}(\tau)$

$=e^{\tau / z} \sum_{a, b, c, d \geq 0} \frac{Q_{1}^{a} Q_{2}^{b} Q_{3}^{c} Q_{4}^{d} e^{a \tau_{1}} e^{b \tau_{2}} e^{c \tau_{3}} e^{d \tau_{4}} \prod_{k=1}^{b+c+d}\left(\lambda+p_{2}+p_{3}+p_{4}+k z\right)}{\prod_{k=1}^{a}\left(p_{1}+k z\right)^{2} \prod_{k=1}^{b}\left(p_{2}+k z\right)^{2} \prod_{k=1}^{c}\left(p_{3}+k z\right)^{2} \prod_{k=1}^{d}\left(p_{4}+k z\right)}$

$$
\times \frac{\prod_{k=-\infty}^{0}\left(p_{4}-p_{1}-p_{2}+k z\right)}{\prod_{k=-\infty}^{d-a-b}\left(p_{4}-p_{1}-p_{2}+k z\right)} .
$$

Note that, much as in Example D.8, we have

$I_{\boldsymbol{e}, E}(0)=1+\left(\left(Q_{3}+Q_{4}+2 Q_{3} Q_{4}\right) 1+\left(p_{4}-p_{1}-p_{2}\right) \log \left(1+Q_{2}\right)\right) z^{-1}+O\left(z^{-2}\right)$.

Arguing exactly as in Example D.8, we find that

$$
J_{\boldsymbol{e}, E}\left(\left(p_{4}-p_{2}-p_{1}\right) \log \left(1+Q_{2}\right)\right)=e^{-\left(Q_{3}+Q_{4}+2 Q_{2} Q_{4}\right) / z} I_{\boldsymbol{e}, E}(0)
$$

and

$$
\begin{aligned}
& J_{\boldsymbol{e}, E}\left(\left(p_{3}-p_{2}-p_{1}\right) \log \left(1+Q_{2}\right)\right) \\
& \quad=e^{\left(p_{4}-p_{2}-p_{1}\right) \log \left(1+Q_{2}\right) / z}\left[J_{\boldsymbol{e}, E}(0)\right]_{Q_{1}=\frac{Q_{1}}{1+Q_{2}}, Q_{2}=\frac{Q_{2}}{1+Q_{2}}, Q_{3}=Q_{3}, Q_{4}=Q_{4}\left(1+Q_{2}\right)}
\end{aligned}
$$

Hence, using the inverse mirror map

$$
Q_{1}=\frac{Q_{1}}{1-Q_{2}}, \quad Q_{2}=\frac{Q_{2}}{1-Q_{2}}, \quad Q_{3}=Q_{3} \quad \text { and } \quad Q_{4}=Q_{4}\left(1-Q_{2}\right),
$$

we have that $J_{\boldsymbol{e}, E}(0)$ is equal to

$$
\begin{aligned}
& {\left[e^{-\left(p_{4}-p_{2}-p_{1}\right) \log \left(1+Q_{2}\right) / z}\right.} \\
& \left.\quad \times J_{\boldsymbol{e}, E}\left(\left(p_{4}-p_{2}-p_{1}\right) \log \left(1+Q_{2}\right)\right)\right]_{Q_{1}=\frac{Q_{1}}{1-Q_{2}}, Q_{2}=\frac{Q_{2}}{1-Q_{2}}, Q_{3}=Q_{3}, Q_{4}=Q_{4}\left(1-Q_{2}\right)} \\
& =e^{\left(p_{4}-p_{2}-p_{1}\right) \log \left(1-Q_{2}\right) / z} \\
& \quad \times\left[e^{-\left(Q_{3}+Q_{4}+2 Q_{2} Q_{4}\right) / z} I_{\boldsymbol{e}, E}(0)\right]_{Q_{1}=\frac{Q_{1}}{1-Q_{2}}, Q_{2}=\frac{Q_{2}}{1-Q_{2}}, Q_{3}=Q_{3}, Q_{4}=Q_{4}\left(1-Q_{2}\right)^{\circ}}
\end{aligned}
$$

Taking the non-equivariant limit yields

$$
J_{Y, X}(0)
$$

$$
\begin{aligned}
&= e^{\left(p_{4}-p_{2}-p_{1}\right) \log \left(1-Q_{2}\right) / z} e^{-\left(Q_{3}+Q_{4}+Q_{2} Q_{4}\right)} \\
& \times \sum_{a, b, c, d \geq 0} \frac{Q_{1}^{a} Q_{2}^{b} Q_{3}^{c} Q_{4}^{d}\left(1-Q_{2}\right)^{d-a-b} \prod_{k=1}^{b+c+d}\left(p_{2}+p_{3}+p_{4}+k z\right)}{\prod_{k=1}^{a}\left(p_{1}+k z\right)^{2} \prod_{k=1}^{b}\left(p_{2}+k z\right)^{2} \prod_{k=1}^{c}\left(p_{3}+k z\right)^{2} \prod_{k=1}^{d}\left(p_{4}+k z\right)} \\
& \times \frac{\prod_{k=-\infty}^{0}\left(p_{4}-p_{1}-p_{2}+k z\right)}{\prod_{k=-\infty}^{d-a-b}\left(p_{4}-p_{1}-p_{2}+k z\right)}
\end{aligned}
$$


We saw in Example D.8 how to obtain the quantum period $G_{X}$ from $J_{Y, X}(0)$ : we extract the component along the unit class $1 \in H^{\bullet}(Y ; \mathbb{Q})$ and set $z=1$ and $Q^{\beta}=t^{\left\langle\beta,-K_{X}\right\rangle}$ (ie set $Q_{1}=Q_{2}=Q_{3}=t$ and $Q_{2}=1$ ). This yields

Regularizing gives

$$
G_{X}(t)=e^{-3 t} \sum_{a=0}^{\infty} \sum_{b=0}^{\infty} \sum_{c=0}^{\infty} t^{2 a+b+c} \frac{(a+2 b+c) !}{(a !)^{2}(b !)^{2}(c !)^{2}(a+b) !} .
$$

$$
\begin{aligned}
\widehat{G}_{X}(t)=1+12 t^{2}+42 t^{3}+468 t^{4}+ & 3360 t^{5}+31350 t^{6} \\
& +275940 t^{7}+2599380 t^{8}+24566640 t^{9}+\cdots .
\end{aligned}
$$

Minkowski period sequence 110

\section{The Fano manifold $\mathrm{MM}_{4-3}$}

Mori-Mukai construction The blow-up of the cone $Y$ over a smooth quadric surface $S$ in $\mathbb{P}^{3}$ with centre the disjoint union of the vertex and an elliptic curve on $S$.

Our construction A member $X$ of $|2 N|$ in the toric variety with weight data

\begin{tabular}{cccccccc}
$s_{0}$ & $s_{1}$ & $t_{0}$ & $t_{1}$ & $x$ & $y_{0}$ & $y_{1}$ & \\
\cline { 1 - 6 } 1 & 1 & 0 & 0 & -1 & 0 & 0 & $L$ \\
0 & 0 & 1 & 1 & -1 & 0 & 0 & $M$ \\
0 & 0 & 0 & 0 & 1 & 1 & 1 & $N$
\end{tabular}

and Nef $F=\langle L, M, N\rangle$. The toric variety $F$ is the same as for $\mathrm{MM}_{3-3}$ and the secondary fan for $F$ is shown in Figure 1 .

We have:

- $-K_{F}=L+M+3 N$ is ample, so $F$ is a Fano variety.

- $\quad X \sim 2 N$ is nef.

- $-\left(K_{F}+X\right) \sim L+M+N$ is ample.

The two constructions coincide The variety $X$ is cut out by

$$
y_{0} y_{1}+x^{2} A_{2,2}\left(s_{0}, s_{1} ; t_{0}, t_{1}\right)=0,
$$

where $A_{2,2}$ is a generic bihomogeneous polynomial of degrees 2 in $s_{0}$ and $s_{1}$, and 2 in $t_{0}$ and $t_{1}$. Note the obvious morphism $\pi: F \rightarrow \mathbb{P}_{s_{0}, s_{1}}^{1} \times \mathbb{P}_{t_{0}, t_{1}}^{1}$ and the morphism $f: F \rightarrow G$ to the double cone $G \subset \mathbb{P}^{5}$ over $\mathbb{P}^{1} \times \mathbb{P}^{1}$ given (contravariantly) by $\left[y_{0}, y_{1}, y_{2}, y_{3}, y_{4}, y_{5}\right] \mapsto\left[y_{0}, y_{1}, s_{0} t_{0} x, s_{0} t_{1} x, s_{1} t_{0} x, s_{1} t_{1} x\right]$. The exceptional set of 
$f$ is the divisor $E=(x=0)=\mathbb{P}_{s_{0}, s_{1}}^{1} \times \mathbb{P}_{t_{0}, t_{1}}^{1} \times \mathbb{P}_{y_{0}, y_{1}}^{1}$ that maps to $\mathbb{P}_{y_{0}, y_{1}}^{1} \subset G$. Note that $E \cap X$ is $t w o$ copies of $\mathbb{P}_{s_{0}, s_{1}}^{1} \times \mathbb{P}_{t_{0}, t_{1}}^{1}$, one above $\left[y_{0}: y_{1}\right]=[1: 0]$ and one above $\left[y_{0}: y_{1}\right]=[0: 1]$. This explains how $X$ has rank 4 when $F$ has rank 3 .

To see that our construction coincides with the construction of Mori and Mukai, set $W=f(X)$, note that

$$
W=\left(y_{0} y_{1}+\tilde{A}_{2}\left(y_{2}, y_{3}, y_{4}, y_{5}\right)=0\right) \subset G
$$

for some degree-2 homogeneous polynomial $\tilde{A}_{2}$ and note that the morphism $f: X \rightarrow W$ contracts one copy of $\mathbb{P}_{s_{0}, s_{1}}^{1} \times \mathbb{P}_{t_{0}, t_{1}}^{1}$, with normal bundle $\mathcal{O}(-1,-1)$, to each of the two singular points $W \cap \mathbb{P}_{y_{0}, y_{1}}^{1}$. Consider next the rational projection $g: G \rightarrow \mathbb{P}_{y_{1}, \ldots, y_{5}}^{4}$ which omits the homogeneous co-ordinate $y_{0}$. It is clear that $\left.g\right|_{W}: W \rightarrow \mathbb{P}^{4}$ is birational onto its image $Y$ (the cone over $\mathbb{P}^{1} \times \mathbb{P}^{1}$ ), that it extends to a morphism after blowing up the singular point $[1: 0: 0: 0: 0: 0] \in W$ and that this morphism contracts the surface $\left(y_{1}=\tilde{A}_{2}\left(y_{2}, y_{3}, y_{4}, y_{5}\right)=0\right) \subset W$ to the elliptic curve $\left(y_{1}=\tilde{A}_{2}\left(y_{2}, y_{3}, y_{4}, y_{5}\right)=0\right) \subset Y$.

The quantum period Corollary D.5 yields

$$
G_{X}(t)=e^{-2 t} \sum_{l=0}^{\infty} \sum_{m=0}^{\infty} \sum_{n=l+m}^{\infty} t^{l+m+n} \frac{(2 n) !}{(l !)^{2}(m !)^{2}(n-l-m) !(n !)^{2}}
$$

and regularizing gives

$$
\begin{aligned}
\widehat{G}_{X}(t)=1+10 t^{2}+24 t^{3}+318 t^{4}+ & 1680 t^{5}+16300 t^{6} \\
+ & 115920 t^{7}+1040830 t^{8}+8403360 t^{9}+\cdots .
\end{aligned}
$$

Minkowski period sequence 88

\section{The Fano manifold $\mathrm{MM}_{4-4}$}

Mori-Mukai construction The blow-up of $\mathbb{P}^{1} \times \mathbb{P}^{1} \times \mathbb{P}^{1}$ with centre a curve $\Gamma$ of tridegree $(1,1,2)$.

Our construction A member $X$ of $|A+B+D|$ in the toric variety $F$ with weight data

\begin{tabular}{rrrrrrrrr}
$x_{0}$ & $x_{1}$ & $y_{0}$ & $y_{1}$ & $z_{0}$ & $z_{1}$ & $u$ & $v$ & \\
\hline 1 & 1 & 0 & 0 & 0 & 0 & 0 & 0 & $A$ \\
0 & 0 & 1 & 1 & 0 & 0 & 0 & 0 & $B$ \\
0 & 0 & 0 & 0 & 1 & 1 & -1 & 0 & $C$ \\
0 & 0 & 0 & 0 & 0 & 0 & 1 & 1 & $D$
\end{tabular}


and Nef $F=\langle A, B, C, D\rangle$. We have:

- $-K_{F}=2 A+2 B+C+2 D$ is ample, that is, $F$ is a Fano variety.

- $X \sim A+B+D$ is nef.

- $-\left(K_{F}+X\right) \sim A+B+C+D$ is ample.

The two constructions coincide We can take $\Gamma \subset \mathbb{P}_{x_{0}, x_{1}}^{1} \times \mathbb{P}_{y_{0}, y_{1}}^{1} \times \mathbb{P}_{z_{0}, z_{1}}^{1}$ to be parameterised as

$$
\left[x_{0}: x_{1}: y_{0}: y_{1}: z_{0}: z_{1}\right] \mapsto\left[s_{0}: s_{1}: s_{0}: s_{1}: s_{0}^{2}: s_{1}^{2}\right] \text {, }
$$

so $\Gamma$ is the complete intersection in $\mathbb{P}^{1} \times \mathbb{P}^{1} \times \mathbb{P}^{1}$ given by the equations $x_{0} y_{1}-x_{1} y_{0}=$ $z_{1} x_{0} y_{0}-z_{0} x_{1} y_{1}=0$. Now apply Lemma E. 1 with

$$
\begin{aligned}
G & =\mathbb{P}^{1} \times \mathbb{P}^{1} \times \mathbb{P}^{1}, \\
V & =\mathcal{O}_{\mathbb{P}^{1} \times \mathbb{P}^{1} \times \mathbb{P}^{1}}(0,0,-1) \oplus \mathcal{O}_{\mathbb{P}^{1} \times \mathbb{P}^{1} \times \mathbb{P}^{1},}, \\
W & =\mathcal{O}_{\mathbb{P}^{1} \times \mathbb{P}^{1} \times \mathbb{P}^{1}}(1,1,0),
\end{aligned}
$$

and $f: V \rightarrow W$ given by the matrix $\left(z_{1} x_{0} y_{0}-z_{0} x_{1} y_{1} x_{0} y_{1}-x_{1} y_{0}\right)$.

The quantum period Corollary D.5 yields

$$
G_{X}(t)=e^{-3 t} \sum_{a=0}^{\infty} \sum_{b=0}^{\infty} \sum_{c=0}^{\infty} \sum_{d=c}^{\infty} t^{a+b+c+d} \frac{(a+b+d) !}{(a !)^{2}(b !)^{2}(c !)^{2}(d-c) ! d !}
$$

and regularizing gives

$$
\begin{aligned}
\widehat{G}_{X}(t)=1+8 t^{2}+24 t^{3}+216 t^{4}+1320 t^{5}+10160 t^{6} \\
+74760 t^{7}+584920 t^{8}+4598160 t^{9}+\cdots .
\end{aligned}
$$

Minkowski period sequence 83

\section{The Fano manifold $\mathrm{MM}_{4-5}$}

Mori-Mukai construction The blow-up of $\mathrm{MM}_{3-19}$ - which is the blow-up of a quadric 3-fold $Q \subset \mathbb{P}^{4}$ with centre two points $P_{1}$ and $P_{2}$ on it which are not collinear with centre the strict transform of a conic containing $P_{1}$ and $P_{2}$. 
Our construction A member $X$ of $|2 N|$ in the toric variety $F$ with weight data

\begin{tabular}{rrrrrrrr}
$s_{0}$ & $s_{1}$ & $x$ & $x_{2}$ & $y$ & $x_{3}$ & $y_{4}$ & \\
\cline { 1 - 4 } & 1 & -1 & 0 & 0 & 0 & 0 & $L$ \\
0 & 0 & 1 & 1 & -1 & 0 & 0 & $M$ \\
0 & 0 & 0 & 0 & 1 & 1 & 1 & $N$
\end{tabular}

and Nef $F=\langle L, M, N\rangle$. We have:

- $-K_{F}=L+M+3 N$ is ample, that is, $F$ is a Fano variety.

- $\quad X \sim 2 N$ is nef.

- $-\left(K_{F}+X\right) \sim L+M+N$ is ample.

The two constructions coincide The complete linear system $|N|$ defines a morphism $F \rightarrow \mathbb{P}^{4}$ which sends (contravariantly) the homogeneous co-ordinate functions $\left[x_{0}, x_{1}, x_{2}, x_{3}, x_{4}\right]$ to

$$
\left[s_{0} x y, s_{1} x y, x_{2} y, x_{3}, x_{4}\right] .
$$

This morphism identifies $F$ with the blow-up of the line $\left(x_{2}=x_{3}=x_{4}=0\right) \subset \mathbb{P}^{4}$ followed by the blow up of the proper transform of the plane $\left(x_{3}=x_{4}=0\right)$. The variety $X$ is the strict transform of a general quadric in $\mathbb{P}^{4}$; in other words, $X$ is a general member of the linear system $|2 N|$ on $F$.

Remark Note that $X$ has rank 4 even though the ambient space $F$ has rank 3; there is no contradiction here because $2 N$ is not ample on $F$.

The quantum period Corollary D.5 yields

$$
G_{X}(t)=e^{-2 t} \sum_{l=0}^{\infty} \sum_{m=l}^{\infty} \sum_{n=m}^{\infty} t^{l+m+n} \frac{(2 n) !}{(l !)^{2}(m-l) ! m !(n-m) !(n !)^{2}}
$$

and regularizing gives

$$
\begin{aligned}
\widehat{G}_{X}(t)=1+6 t^{2}+24 t^{3}+138 t^{4}+960 t^{5} & +6180 t^{6} \\
+ & 43680 t^{7}+311850 t^{8}+2274720 t^{9}+\cdots .
\end{aligned}
$$

Minkowski period sequence 68

\section{The Fano manifold $\mathrm{MM}_{4-6}$}

Mori-Mukai construction The blow-up of $\mathbb{P}^{2} \times \mathbb{P}^{1}$ with centre two disjoint curves, one of bidegree $(1,2)$ and the other of bidegree $(0,1)$. 
Our construction A member $X$ of $|C+D|$ in the toric variety with weight data

\begin{tabular}{rrrrrrrrr}
$s_{0}$ & $s_{1}$ & $t_{0}$ & $t_{1}$ & $x$ & $x_{2}$ & $u$ & $v$ & \\
\hline 1 & 1 & 0 & 0 & -1 & 0 & -1 & 0 & $A$ \\
0 & 0 & 1 & 1 & 0 & 0 & -1 & 0 & $B$ \\
0 & 0 & 0 & 0 & 1 & 1 & 0 & 0 & $C$ \\
0 & 0 & 0 & 0 & 0 & 0 & 1 & 1 & $D$
\end{tabular}

and Nef $F=\langle A, B, C, D\rangle$. We have:

- $-K_{F}=B+2 C+2 D$ is nef and big but not ample.

- $\quad X \sim C+D$ is nef and big but not ample.

- $-\left(K_{F}+X\right) \sim B+C+D$ is nef and big but not ample.

The two constructions coincide The variety $X$ is cut out by

$$
v x_{2}+u x A_{2,1}\left(s_{0}, s_{1} ; t_{0}, t_{1}\right)=0 \text {. }
$$

Note the obvious morphism $\pi: F \rightarrow G$ with fibre $\mathbb{P}_{u, v}^{1}$, where $G$ is the toric variety with weight data

\begin{tabular}{rrrrrrr}
$s_{0}$ & $s_{1}$ & $t_{0}$ & $t_{1}$ & $x$ & $x_{2}$ & \\
\cline { 1 - 5 } 1 & 1 & 0 & 0 & -1 & 0 & $A$ \\
0 & 0 & 1 & 1 & 0 & 0 & $B$ \\
0 & 0 & 0 & 0 & 1 & 1 & $C$
\end{tabular}

and Nef $G=\langle A, B, C\rangle$. The birational morphism $G \rightarrow \mathbb{P}_{x_{0}, x_{1}, x_{2}}^{2} \times \mathbb{P}_{t_{0}, t_{1}}^{1}$ given (contravariantly) by $\left[x_{0}, x_{1}, x_{2}, t_{0}, t_{1}\right] \mapsto\left[s_{0} x, s_{1} x, x_{2}, t_{0}, t_{1}\right]$ identifies $G$ with the blow-up of the curve $\{[0: 0: 1]\} \times \mathbb{P}^{1} \subset \mathbb{P}^{2} \times \mathbb{P}^{1}$; this curve has bidegree $(0,1)$. The equation defining $X$ has degree 1 in $\mathbb{P}_{u, v}^{1}$; it follows that the morphism $\left.\pi\right|_{X}: X \rightarrow G$ is birational and blows up the locus ${ }^{19}\left(x_{2}=A_{2,1}\left(s_{0}, s_{1} ; t_{0}, t_{1}\right)=0\right) \subset G$.

The quantum period Let $p_{1}, p_{2}, p_{3}, p_{4} \in H^{\bullet}(F ; \mathbb{Z})$ denote the first Chern classes of $A, B, C$ and $D$, respectively; these classes form a basis for $H^{2}(F ; \mathbb{Z})$. Write $\tau \in H^{2}(F ; \mathbb{Q})$ as $\tau=\tau_{1} p_{1}+\tau_{2} p_{2}+\tau_{3} p_{3}+\tau_{4} p_{4}$ and identify the group ring $\mathbb{Q}\left[H_{2}(F ; \mathbb{Z})\right]$ with the polynomial ring $\mathbb{Q}\left[Q_{1}, Q_{2}, Q_{3}, Q_{4}\right]$ via the $\mathbb{Q}$-linear map that

${ }^{19}$ With our choice of stability condition for $F,\left(x_{2}=x=0\right) \subset \mathbb{C}^{8}$ is part of the unstable locus. 
sends the element $Q^{\beta} \in \mathbb{Q}\left[H_{2}(F ; \mathbb{Z})\right]$ to $Q_{1}^{\left\langle\beta, p_{1}\right\rangle} Q_{2}^{\left\langle\beta, p_{2}\right\rangle} Q_{3}^{\left\langle\beta, p_{3}\right\rangle} Q_{4}^{\left\langle\beta, p_{4}\right\rangle}$. We have $I_{F}(\tau)$

$$
\begin{array}{r}
=e^{\tau / z} \sum_{a, b, c, d \geq 0} \frac{Q_{1}^{a} Q_{2}^{b} Q_{3}^{c} Q_{4}^{d} e^{a \tau_{1}} e^{b \tau_{2}} e^{c \tau_{3}} e^{d \tau_{4}}}{\prod_{k=1}^{a}\left(p_{1}+k z\right)^{2} \prod_{k=1}^{b}\left(p_{2}+k z\right)^{2} \prod_{k=1}^{c}\left(p_{3}+k z\right) \prod_{k=1}^{d}\left(p_{4}+k z\right)} \\
\times \frac{\prod_{k=-\infty}^{0}\left(p_{3}-p_{1}+k z\right)}{\prod_{k=-\infty}^{c-a}\left(p_{3}-p_{1}+k z\right)} \frac{\prod_{k=-\infty}^{0}\left(p_{4}-p_{1}-p_{2}+k z\right)}{\prod_{k=-\infty}^{d-a-b}\left(p_{4}-p_{1}-p_{2}+k z\right)} .
\end{array}
$$

Since

$$
I_{F}(\tau)=1+\tau z^{-1}+O\left(z^{-2}\right),
$$

Theorem C.1 gives

$$
J_{F}(\tau)=I_{F}(\tau)
$$

We now proceed exactly as in the case of $\mathrm{MM}_{3-1}$, obtaining

$$
G_{X}(t)=e^{-2 t} \sum_{a=0}^{\infty} \sum_{b=0}^{\infty} \sum_{c=a}^{\infty} \sum_{d=a+b}^{\infty} t^{b+c+d} \frac{(c+d) !}{(a !)^{2}(b !)^{2} c ! d !(c-a) !(d-a-b) !} .
$$

Regularizing gives

$$
\begin{aligned}
\widehat{G}_{X}(t)=1+8 t^{2}+18 t^{3}+192 t^{4}+960 t^{5} & +7550 t^{6} \\
& +49980 t^{7}+374080 t^{8}+2741760 t^{9}+\cdots .
\end{aligned}
$$

Minkowski period sequence 81

\section{The Fano manifold $\mathrm{MM}_{4-7}$}

Mori-Mukai construction The blow-up of $\mathbb{P}^{1} \times \mathbb{P}^{1} \times \mathbb{P}^{1}$ with centre the curve of tridegree $(1,1,1)$.

Our construction A codimension- 2 complete intersection $X$ of type $D \cap D$ in the toric variety $F$ with weight data

\begin{tabular}{rrrrrrrrrl}
$x_{0}$ & $x_{1}$ & $y_{0}$ & $y_{1}$ & $z_{0}$ & $z_{1}$ & $u_{0}$ & $u_{1}$ & $u_{2}$ & \\
\hline 1 & 1 & 0 & 0 & 0 & 0 & -1 & 0 & 0 & $A$ \\
0 & 0 & 1 & 1 & 0 & 0 & 0 & -1 & 0 & $B$ \\
0 & 0 & 0 & 0 & 1 & 1 & 0 & 0 & -1 & $C$ \\
0 & 0 & 0 & 0 & 0 & 0 & 1 & 1 & 1 & $D$
\end{tabular}

and Nef $F=\langle A, B, C, D\rangle$. We have:

- $-K_{F}=A+B+C+3 D$ is ample, that is, $F$ is a Fano variety. 
- $\quad X$ is complete intersection of two nef divisors on $F$.

- $-\left(K_{F}+\Lambda\right) \sim A+B+C+D$ is ample.

The two constructions coincide Without loss of generality, the curve to be blown up is defined in $\mathbb{P}_{x_{0}, x_{1}}^{1} \times \mathbb{P}_{y_{0}, y_{1}}^{1} \times \mathbb{P}_{z_{0}, z_{1}}^{1}$ by the condition

$$
\operatorname{rk}\left(\begin{array}{lll}
x_{0} & y_{0} & z_{0} \\
x_{1} & y_{1} & z_{1}
\end{array}\right)<2
$$

Now apply Lemma E.1 with

$$
\begin{aligned}
G & =\mathbb{P}^{1} \times \mathbb{P}^{1} \times \mathbb{P}^{1}, \\
V & =\mathcal{O}_{\mathbb{P}^{1} \times \mathbb{P}^{1} \times \mathbb{P}^{1}}(-1,0,0) \oplus \mathcal{O}_{\mathbb{P}^{1} \times \mathbb{P}^{1} \times \mathbb{P}^{1}}(0,-1,0) \oplus \mathcal{O}_{\mathbb{P}^{1} \times \mathbb{P}^{1} \times \mathbb{P}^{1}}(0,0,-1), \\
W & =\mathcal{O}_{\mathbb{P}^{1} \times \mathbb{P}^{1} \times \mathbb{P}^{1}} \oplus \mathcal{O}_{\mathbb{P}^{1} \times \mathbb{P}^{1} \times \mathbb{P}^{1},}
\end{aligned}
$$

and the map $f: V \rightarrow W$ given by the matrix

$$
\left(\begin{array}{lll}
x_{0} & y_{0} & z_{0} \\
x_{1} & y_{1} & z_{1}
\end{array}\right)
$$

The quantum period Corollary D.5 yields

$G_{X}(t)=e^{-t} \sum_{a=0}^{\infty} \sum_{b=0}^{\infty} \sum_{c=0}^{\infty} \sum_{d=\max (a, b, c)}^{\infty} t^{a+b+c+d} \frac{(d !)^{2}}{(a !)^{2}(b !)^{2}(c !)^{2}(d-a) !(d-b) !(d-c) !}$

and regularizing gives

$$
\begin{aligned}
\widehat{G}_{X}(t)=1+6 t^{2}+18 t^{3}+114 t^{4}+720 t^{5} & +4290 t^{6} \\
+ & 28980 t^{7}+193410 t^{8}+1320480 t^{9}+\cdots .
\end{aligned}
$$

Minkowski period sequence 65

\section{The Fano manifold $\mathrm{MM}_{4-8}$}

Mori-Mukai construction The blow-up of $W$ (or $\mathrm{MM}_{2-32}$, a divisor of bidegree $(1,1)$ in $\left.\mathbb{P}^{2} \times \mathbb{P}^{2}\right)$ with centre two disjoint curves on it of bidegrees $(0,1)$ and $(1,0)$. 
Our construction A member $X$ of $|B+D|$ in the toric variety $F$ with weight data

\begin{tabular}{rrrrrrrrr}
$s_{0}$ & $s_{1}$ & $x$ & $x_{2}$ & $t_{0}$ & $t_{1}$ & $y$ & $y_{2}$ & \\
\cline { 1 - 3 } & 1 & -1 & 0 & 0 & 0 & 0 & 0 & $A$ \\
0 & 0 & 1 & 1 & 0 & 0 & 0 & 0 & $B$ \\
0 & 0 & 0 & 0 & 1 & 1 & -1 & 0 & $C$ \\
0 & 0 & 0 & 0 & 0 & 0 & 1 & 1 & $D$
\end{tabular}

and Nef $F=\langle A, B, C, D\rangle$. We have:

- $-K_{F}=A+2 B+C+2 D$ is ample, that is, $F$ is a Fano variety.

- $\quad X \sim B+D$ is nef.

- $-\left(K_{F}+X\right) \sim A+B+C+D$ is ample.

The two constructions coincide We take $W$ to be the divisor:

$$
W=\left(x_{0} y_{0}+x_{1} y_{1}+x_{2} y_{2}=0\right) \subset \mathbb{P}_{x_{0}, x_{1}, x_{2}}^{2} \times \mathbb{P}_{y_{0}, y_{1}, y_{2}}^{2}
$$

It is clear that the morphism $f: F \rightarrow \mathbb{P}^{2} \times \mathbb{P}^{2}$ which sends (contravariantly)

$$
\left[x_{0}, x_{1}, x_{2}, y_{0}, y_{1}, y_{2}\right] \mapsto\left[s_{0} x, s_{1} x, x_{2}, t_{0} y, t_{1} y, y_{2}\right]
$$

blows up the disjoint union of $\left(x_{0}=x_{1}=0\right)$ and $\left(y_{0}=y_{1}=0\right)$ in $\mathbb{P}^{2} \times \mathbb{P}^{2}$. This morphism induces the required blow-up of $W$.

The quantum period Corollary D.5 yields

$$
G_{X}(t)=e^{-2 t} \sum_{a=0}^{\infty} \sum_{b=a}^{\infty} \sum_{c=0}^{\infty} \sum_{d=c}^{\infty} t^{a+b+c+d} \frac{(b+d) !}{(a !)^{2}(b-a) ! b !(c !)^{2}(d-c) ! d !}
$$

and regularizing gives

$$
\begin{aligned}
\widehat{G}_{X}(t)=1+6 t^{2}+12 t^{3}+114 t^{4}+480 t^{5} & +3480 t^{6} \\
& +19320 t^{7}+131250 t^{8}+819840 t^{9}+\cdots .
\end{aligned}
$$

Minkowski period sequence 57

\section{The Fano manifold $\mathrm{MM}_{4-9}$}

Mori-Mukai construction The blow-up of $\mathbb{P}^{1} \times \mathbb{P}^{1} \times \mathbb{P}^{1}$ with centre a curve of tridegree $(0,1,1)$. 
Our construction A member $X$ of $|D|$ in the toric variety $F$ with weight data

\begin{tabular}{rcccccccc}
$x_{0}$ & $x_{1}$ & $y_{0}$ & $y_{1}$ & $z_{0}$ & $z_{1}$ & $u$ & $v$ & \\
\cline { 1 - 4 } & 1 & 0 & 0 & 0 & 0 & 0 & -1 & $A$ \\
0 & 0 & 1 & 1 & 0 & 0 & 0 & -1 & $B$ \\
0 & 0 & 0 & 0 & 1 & 1 & -1 & 0 & $C$ \\
0 & 0 & 0 & 0 & 0 & 0 & 1 & 1 & $D$
\end{tabular}

and Nef $F=\langle A, B, C, D\rangle$. We have:

- $-K_{F}=A+B+C+2 D$ is ample, that is, $F$ is a Fano variety.

- $\quad X \sim D$ is nef.

- $-\left(K_{F}+X\right) \sim A+B+C+D$ is ample.

The two constructions coincide The curve to be blown up is the complete intersection

$$
\left(z_{0}=x_{0} y_{0}+x_{1} y_{1}=0\right) \subset \mathbb{P}_{x_{0}, x_{1}}^{1} \times \mathbb{P}_{y_{0}, y_{1}}^{1} \times \mathbb{P}_{z_{0}, z_{1}}^{1} .
$$

We apply Lemma E.1 with

$$
\begin{aligned}
G & =\mathbb{P}^{1} \times \mathbb{P}^{1} \times \mathbb{P}^{1}, \\
V & =\mathcal{O}_{\mathbb{P}^{1} \times \mathbb{P}^{1} \times \mathbb{P}^{1}}(0,0,-1) \oplus \mathcal{O}_{\mathbb{P}^{1} \times \mathbb{P}^{1} \times \mathbb{P}^{1}}(-1,-1,0), \\
W & =\mathcal{O}_{\mathbb{P}^{1} \times \mathbb{P}^{1} \times \mathbb{P}^{1},}
\end{aligned}
$$

and the map $f: V \rightarrow W$ given by the matrix $\left(z_{0} x_{0} y_{0}+x_{1} y_{1}\right)$.

The quantum period Corollary D.5 yields

$G_{X}(t)=e^{-t} \sum_{a=0}^{\infty} \sum_{b=0}^{\infty} \sum_{c=0}^{\infty} \sum_{d=\max (a+b, c)}^{\infty} t^{a+b+c+d} \frac{d !}{(a !)^{2}(b !)^{2}(c !)^{2}(d-c) !(d-a-b) !}$

and regularizing gives

$\widehat{G}_{X}(t)=1+6 t^{2}+12 t^{3}+90 t^{4}+480 t^{5}+2400 t^{6}+16800 t^{7}+88410 t^{8}+608160 t^{9}+\cdots$.

Minkowski period sequence 54

\section{The Fano manifold $M_{4-10}$}

Mori-Mukai construction The blow-up of $\mathrm{MM}_{3-25}$ - which is the blow-up of $\mathbb{P}^{3}$ with centre two disjoint lines - with centre an exceptional line of the blow-up $\mathrm{MM}_{3-25} \rightarrow \mathbb{P}^{3}$. 
Our construction The toric variety $X$ with weight data

\begin{tabular}{rrrrrrrr}
$s_{0}$ & $s_{1}$ & $t_{2}$ & $t_{3}$ & $x$ & $y$ & $z$ & \\
\hline 1 & 1 & 0 & 0 & -1 & 0 & 0 & $A$ \\
0 & 0 & 1 & 1 & 0 & -1 & 0 & $B$ \\
0 & 0 & 0 & -1 & 0 & 1 & 1 & $C$ \\
0 & 0 & 0 & 1 & 1 & 0 & -1 & $D$
\end{tabular}

and Nef $X=\langle A, B, C, D\rangle$.

The two constructions coincide The morphism $X \rightarrow \mathbb{P}^{3}$ is given by the complete linear system $|C|$. It sends (contravariantly) the homogeneous co-ordinate functions $\left[x_{0}, x_{1}, x_{2}, x_{3}\right]$ to $\left[s_{0} x z, s_{1} x z, t_{2} y, t_{3} y z\right]$. The morphism blows up first the lines $\left(x_{0}=x_{1}=0\right)$ (the image of the divisor $x=0$ in $\left.X\right)$ and $\left(x_{2}=x_{3}=0\right)$ (the image of the divisor $y=0$ in $X$ ), and then the fibre over the point $[0: 0: 1: 0]$ (the image of the divisor $z=0$ in $X$ ).

The quantum period Corollary C.2 yields

$$
G_{X}(t)=\sum_{a=0}^{\infty} \sum_{b=0}^{\infty} \sum_{d=a}^{\infty} \sum_{c=\max (b, d)}^{b+d} \frac{t^{a+b+c+d}}{(a !)^{2} b !(b-c+d) !(d-a) !(c-b) !(c-d) !}
$$

and regularizing gives

$\widehat{G}_{X}(t)=1+4 t^{2}+12 t^{3}+60 t^{4}+300 t^{5}+1660 t^{6}+8820 t^{7}+51100 t^{8}+293160 t^{9}+\cdots$.

Minkowski period sequence 37

\section{The Fano manifold $\mathrm{MM}_{4-11}$}

Mori-Mukai construction $S_{7} \times \mathbb{P}^{1}$.

Our construction $S_{7} \times \mathbb{P}^{1}$.

The quantum period Combining Corollary E.4 with Examples G.1 and G.5 yields

$$
G_{X}(t)=\sum_{a \geq 0} \sum_{b \geq 0} \sum_{c=\max (a, b)}^{a+b} \sum_{d \geq 0} \frac{t^{a+b+c+2 d}}{a ! b !(a+b-c) !(c-a) !(c-b) !(d !)^{2}} .
$$

Regularizing gives

$$
\widehat{G}_{X}(t)=1+6 t^{2}+6 t^{3}+90 t^{4}+240 t^{5}+1950 t^{6}+8400 t^{7}+53130 t^{8}+288960 t^{9}+\cdots .
$$


Minkowski period sequence 48

\section{The Fano manifold $\mathrm{MM}_{4-12}$}

Mori-Mukai construction The blow-up of $\mathbb{P}^{1} \times \mathbb{F}_{1}$ with centre $t \times e$, where $t \in \mathbb{P}^{1}$ and $e$ is the exceptional curve on $\mathbb{F}_{1}$.

Our construction The toric variety $X$ with weight data

\begin{tabular}{rrrrrrrr}
$y_{0}$ & $y_{1}^{\prime}$ & $s_{0}$ & $s_{1}$ & $x^{\prime}$ & $x_{2}$ & $w$ & \\
\cline { 1 - 5 } & 0 & 0 & 0 & -1 & 0 & 1 & $A$ \\
0 & 0 & 1 & 1 & -1 & 0 & 0 & $B$ \\
0 & -1 & 0 & 0 & 0 & 1 & 1 & $C$ \\
0 & 1 & 0 & 0 & 1 & 0 & -1 & $D$
\end{tabular}

and Nef $X=\langle A, B, C, D\rangle$.

The two constructions coincide Let $\left[y_{0}: y_{1}\right]$ be homogeneous co-ordinates on $\mathbb{P}^{1}$, and recall that $\mathbb{F}_{1}$ is the toric variety with weight data:

\begin{tabular}{rrrrr}
$s_{0}$ & $s_{1}$ & $x$ & $x_{2}$ & \\
\cline { 1 - 3 } 1 & 1 & -1 & 0 & $L$ \\
0 & 0 & 1 & 1 & $M$
\end{tabular}

The morphism $X \rightarrow \mathbb{P}^{1} \times \mathbb{F}_{1}$ is given (contravariantly) by

$$
\left[y_{0}, y_{1}, s_{0}, s_{1}, x, x_{2}\right] \mapsto\left[y_{0}, y_{1}^{\prime} w, s_{0}, s_{1}, x^{\prime} w, x_{2}\right] .
$$

The quantum period Corollary C.2 yields

$$
G_{X}(t)=\sum_{a=0}^{\infty} \sum_{b=0}^{\infty} \sum_{c=0}^{\infty} \sum_{d=\max (a+b, c)}^{a+c} \frac{t^{a+b+c+d}}{a !(d-c) !(b !)^{2}(d-a-b) ! c !(a+c-d) !}
$$

and regularizing gives

$\widehat{G}_{X}(t)=1+4 t^{2}+12 t^{3}+36 t^{4}+300 t^{5}+940 t^{6}+6300 t^{7}+31780 t^{8}+157080 t^{9}+\cdots$.

Minkowski period sequence 34

\section{The Fano manifold $\mathrm{MM}_{4-13}$}

Mori-Mukai construction The blow-up of $\mathrm{MM}_{2-33}$ - which is the blow-up of $\mathbb{P}^{3}$ with centre a line — with centre two exceptional lines of the blow-up $\mathrm{MM}_{2-33} \rightarrow \mathbb{P}^{3}$. 
Our construction The toric variety $X$ with weight data

\begin{tabular}{rrrrrrrr}
$s_{0}$ & $s_{1}$ & $x$ & $y_{2}$ & $y_{3}$ & $u$ & $v$ & \\
\cline { 1 - 5 } 1 & 1 & -1 & 0 & 0 & 0 & 0 & $A$ \\
0 & 0 & -1 & 0 & 0 & 1 & 1 & $B$ \\
0 & 0 & 1 & 1 & 0 & -1 & 0 & $C$ \\
0 & 0 & 1 & 0 & 1 & 0 & -1 & $D$
\end{tabular}

and Nef $X=\langle A, B, C, D\rangle$.

The two constructions coincide Recall that $\mathrm{MM}_{2-33}$ is the toric variety with weight data

\begin{tabular}{rrrrr}
$s_{0}$ & $s_{1}$ & $x$ & $x_{2}$ & $x_{3}$ \\
\hline 1 & 1 & -1 & 0 & 0 \\
0 & 0 & 1 & 1 & 1
\end{tabular}

and the morphism $\mathrm{MM}_{2-33} \rightarrow \mathbb{P}^{3}$ sends (contravariantly) the homogeneous co-ordinate functions $\left[x_{0}, x_{1}, x_{2}, x_{3}\right]$ on $\mathbb{P}^{3}$ to $\left[s_{0} x, s_{1} x, x_{2}, x_{3}\right]$. The blow-up $X \rightarrow \mathrm{MM}_{2-33}$ is given (again contravariantly) by $\left[s_{0}, s_{1}, x, x_{2}, x_{3}\right] \mapsto\left[s_{0}, s_{1}, u v x, u x_{2}, v x_{3}\right]$.

The quantum period Corollary C.2 yields

$$
G_{X}(t)=\sum_{a=0}^{\infty} \sum_{b=0}^{\infty} \sum_{c=0}^{b} \sum_{d=\max (0, a+b-c)}^{b} \frac{t^{a+b+c+d}}{(a !)^{2}(c+d-a-b) ! c ! d !(b-c) !(b-d) !}
$$

and regularizing gives

$\widehat{G}_{X}(t)=1+4 t^{2}+6 t^{3}+60 t^{4}+120 t^{5}+1210 t^{6}+3360 t^{7}+27580 t^{8}+97440 t^{9}+\cdots$.

Minkowski period sequence 29

\section{The Fano manifold $\mathrm{MM}_{5-1}$}

Mori-Mukai construction The blow-up of $\mathrm{MM}_{2-29}$ - which is the blow-up of a quadric 3-fold $Q \subset \mathbb{P}^{3}$ with centre a conic on it - with centre three exceptional lines of the blow-up $\mathrm{MM}_{2-29} \rightarrow Q$. 
Our construction A member $X$ of $|2 A+2 B+C+D+E|$ in the toric variety $F$ with weight data

\begin{tabular}{|c|c|c|c|c|c|c|c|c|}
\hline 1 & 1 & 1 & 1 & 1 & 0 & 0 & 0 & 0 \\
\hline 1 & 1 & 1 & 0 & 0 & 1 & 0 & 0 & 0 \\
\hline 1 & 0 & 0 & 0 & 0 & 0 & 1 & 0 & 0 \\
\hline 0 & 1 & 0 & 0 & 0 & 0 & 0 & 1 & 0 \\
\hline 0 & 0 & 1 & 0 & 0 & 0 & 0 & 0 & 1 \\
\hline
\end{tabular}

and

Nef $F=\langle A, A+B+D+E, A+B+C+E, A+B+C+D$,

$$
A+B+C+D+E, 2 A+2 B+C+D+E\rangle \text {. }
$$

We have:

- $-K_{F}=5 A+4 B+2 C+2 D+2 E=2(2 A+2 B+C+D+E)+(A)$ is nef and big but not ample.

- $X \sim 2 A+2 B+C+D+E$ is nef.

- $-\left(K_{F}+X\right) \sim 3 A+2 B+C+D+E$ is nef and big but not ample.

The two constructions coincide There is a morphism ${ }^{20} F \rightarrow \mathbb{P}^{4}$ given by the complete linear system $|A+B+C+D+E|$; it sends (contravariantly) the homogeneous co-ordinate functions $\left[x_{0}, x_{1}, x_{2}, x_{3}, x_{4}\right]$ on $\mathbb{P}^{4}$ to

$$
\left[z_{0} t_{02} t_{01}, z_{1} t_{12} t_{01}, z_{2} t_{12} t_{02}, s_{3} x t_{12} t_{02} t_{01}, s_{3} x t_{12} t_{02} t_{01}\right] \text {. }
$$

This morphism can be factorized by first blowing up the plane $\Pi=\left(x_{3}=x_{4}=0\right) \subset \mathbb{P}^{4}$, and subsequently blowing up the three fibres over the co-ordinate points

$$
P_{0}=[1: 0: 0: 0: 0], \quad P_{1}=[0: 1: 0: 0: 0] \quad \text { and } \quad P_{2}=[0: 0: 1: 0: 0]
$$

in $\Pi$. Thus we can take $X$ to be the proper transform of any quadric $Q \subset \mathbb{P}^{4}$ containing the three points $P_{0}, P_{1}$ and $P_{2}$ but not containing the plane $\Pi$, for instance the quadric given by the equation

$$
x_{0} x_{1}+x_{1} x_{2}+x_{2} x_{0}+x_{3}^{2}+x_{4}^{2}=0 .
$$

${ }^{20}$ The class $-K_{F}$ belongs to 7 simplicial cones and a non-simplicial cone (the one that we chose to be Nef $F$ ). It turns out that the class $2 A+2 B+C+D+E$ also belongs to all of these cones. However, only one of these cones contains $A+B+C+D+E$; this is the cone that we chose to be Nef $F$. 
The quantum period Corollary D.5 yields

$$
\begin{aligned}
G_{X}(t)=e^{-3 t} \sum_{a=0}^{\infty} \sum_{b=0}^{\infty} \sum_{c=0}^{\infty} \sum_{d=0}^{\infty} \sum_{e=0}^{\infty} t^{3 a+2 b+c+d+e} & \times \frac{(2 a+2 b+c+d+e) !}{(a+b+c) !(a+b+d) !(a+b+e) !(a !)^{2} b ! c ! d ! e !}
\end{aligned}
$$

and regularizing gives

$$
\begin{aligned}
\widehat{G}_{X}(t)=1+10 t^{2}+42 t^{3}+342 t^{4}+ & 2640 t^{5}+21250 t^{6} \\
& +180600 t^{7}+1562470 t^{8}+13851600 t^{9}+\cdots .
\end{aligned}
$$

Minkowski period sequence 100

\section{The Fano manifold $\mathrm{MM}_{5-2}$}

Mori-Mukai construction The blow-up of $\mathrm{MM}_{3-25}$ - which is the blow-up of $\mathbb{P}^{3}$ with centre two disjoint lines - with centre two exceptional lines $\ell$ and $\ell^{\prime}$ of the blow-up $f: M_{3-25} \rightarrow \mathbb{P}^{3}$ such that $\ell$ and $\ell^{\prime}$ lie on the same irreducible component of the exceptional set of $f$.

Our construction The toric variety $X$ with weight data

\begin{tabular}{rrrrrrrrr}
$s_{0}$ & $s_{1}$ & $t_{2}$ & $t_{3}$ & $x$ & $y$ & $u$ & $v$ & \\
\cline { 1 - 2 }$n$ & 1 & 0 & 0 & -1 & 0 & 0 & 0 & $A$ \\
0 & 0 & 1 & 1 & 0 & -1 & 0 & 0 & $B$ \\
0 & 0 & 0 & 1 & 1 & 0 & -1 & 0 & $C$ \\
0 & 0 & 1 & 0 & 1 & 0 & 0 & -1 & $D$ \\
0 & 0 & -1 & -1 & -1 & 1 & 1 & 1 & $E$
\end{tabular}

and Nef $X=\langle A, B, C, D, E, B+C+D-E\rangle$.

The two constructions coincide Consider the morphism $f: X \rightarrow \mathbb{P}^{3}$ given by the complete linear system $E$. The morphism $f$ sends (contravariantly) the homogeneous co-ordinate functions $\left[x_{0}, x_{1}, x_{2}, x_{3}\right]$ on $\mathbb{P}^{3}$ to $\left[s_{0} x u v, s_{1} x u v, t_{2} y v, t_{3} y u\right]$; it contracts

- the divisors $(x=0)$ and $(y=0)$ to the lines $x_{0}=x_{1}=0$ and $x_{2}=x_{3}=0$, and

- the divisors $(u=0)$ and $(v=0)$ to the points $P_{0}=[0: 0: 0: 1]$ and $P_{1}=$ $[0: 0: 1: 0]$. 
The quantum period Corollary D.5 yields $G_{X}(t)=\sum_{a=0}^{\infty} \sum_{b=0}^{\infty} \sum_{c=0}^{\infty} \sum_{d=0}^{\infty} \sum_{e=\max (b, c, d)}^{\min (b+c, b+d, c+d-a)} \frac{t^{a+b+c+d}}{(a !)^{2}(b+d-e) !(b+c-e) !(c+d-a-e) !}$ $\times \frac{1}{(e-b) !(e-c) !(e-d) !}$

and regularizing gives

$$
\begin{aligned}
\widehat{G}_{X}(t)=1+6 t^{2}+18 t^{3}+114 t^{4}+660 t^{5} & +3930 t^{6} \\
& +25620 t^{7}+163170 t^{8}+1101240 t^{9}+\cdots .
\end{aligned}
$$

Minkowski period sequence 64

\section{The Fano manifold $\mathrm{MM}_{5-3}$}

Mori-Mukai construction $S_{6} \times \mathbb{P}^{1}$.

Our construction $S_{6} \times \mathbb{P}^{1}$.

The quantum period Combining Corollary E.4 with Examples G.1 and G.6 yields

$$
G_{X}(t)=\sum_{a=0}^{\infty} \sum_{b=0}^{\infty} \sum_{c=0}^{\infty} \sum_{d=\max (a-c, 0)}^{a+b} \sum_{e=0}^{\infty} \frac{t^{a+2 b+2 c+d+2 e}}{a ! b ! c ! d !(a+b-d) !(c+d-a) !(e !)^{2}} .
$$

Regularizing gives

$$
\begin{aligned}
\widehat{G}_{X}(t)=1+8 t^{2}+12 t^{3}+168 t^{4}+600 t^{5} & +5300 t^{6} \\
+ & 27720 t^{7}+210280 t^{8}+1308720 t^{9}+\cdots .
\end{aligned}
$$

Minkowski period sequence 76

\section{The Fano manifold $\mathrm{MM}_{6-1}$}

Mori-Mukai construction $S_{5} \times \mathbb{P}^{1}$.

Our construction $S_{5} \times \mathbb{P}^{1}$. 
The quantum period Combining Corollary E.4 with Examples G.1 and G.7 yields

Regularizing gives

$$
G_{X}(t)=e^{-3 t} \sum_{l=0}^{\infty} \sum_{m=0}^{\infty} \sum_{n=0}^{\infty} t^{l+m+2 n} \frac{(l+2 m) !}{(l !)^{2}(m !)^{3}(n !)^{2}} .
$$

$$
\begin{aligned}
\widehat{G}_{X}(t)=1+12 t^{2}+30 t^{3}+396 t^{4}+ & 2160 t^{5}+20370 t^{6} \\
& +149520 t^{7}+1315020 t^{8}+10864560 t^{9}+\cdots .
\end{aligned}
$$

Minkowski period sequence 107

\section{The Fano manifold $\mathrm{MM}_{7-1}$}

Mori-Mukai construction $S_{4} \times \mathbb{P}^{1}$.

Our construction $S_{4} \times \mathbb{P}^{1}$.

The quantum period Combining Corollary E.4 with Examples G.1 and G.8 yields

Regularizing gives

$$
G_{X}(t)=e^{-4 t} \sum_{l=0}^{\infty} \sum_{m=0}^{\infty} t^{l+2 m} \frac{(2 l) !(2 l) !}{(l !)^{5}(m !)^{2}} .
$$

$$
\begin{aligned}
\widehat{G}_{X}(t)=1+22 t^{2}+96 t^{3}+1434 t^{4} & +12480 t^{5}+148900 t^{6} \\
& +1606080 t^{7}+18905530 t^{8}+220617600 t^{9}+\cdots .
\end{aligned}
$$

Minkowski period sequence 136

\section{The Fano manifold $\mathrm{MM}_{8-1}$}

Mori-Mukai construction $S_{3} \times \mathbb{P}^{1}$.

Our construction $S_{3} \times \mathbb{P}^{1}$.

The quantum period Combining Corollary E.4 with Examples G.1 and G.9 yields

Regularizing gives

$$
G_{X}(t)=e^{-6 t} \sum_{l=0}^{\infty} \sum_{m=0}^{\infty} t^{l+2 m} \frac{(3 l) !}{(l !)^{4}(m !)^{2}} .
$$

$$
\begin{aligned}
\widehat{G}_{X}(t)=1+56 t^{2}+492 t^{3} & +10536 t^{4}+168600 t^{5}+3180980 t^{6} \\
+ & 58753800 t^{7}+1129788520 t^{8}+21955158960 t^{9}+\cdots .
\end{aligned}
$$


Minkowski period sequence 155

\section{The Fano manifold MM9-1}

Mori-Mukai construction $S_{2} \times \mathbb{P}^{1}$.

Our construction $S_{2} \times \mathbb{P}^{1}$.

The quantum period Combining Corollary E.4 with Examples G.1 and G.10 yields

Regularizing gives

$$
G_{X}(t)=e^{-12 t} \sum_{l=0}^{\infty} \sum_{m=0}^{\infty} t^{l+2 m} \frac{(4 l) !}{(l !)^{3}(2 l) !(m !)^{2}} .
$$

$$
\begin{aligned}
\widehat{G}_{X}(t)=1+278 t^{2}+6816 t^{3}+317850 t^{4}+12989760 t^{5}+578870180 t^{6} \\
+26074520640 t^{7}+1202038745530 t^{8}+56188933046400 t^{9}+\cdots
\end{aligned}
$$

Minkowski period sequence None. Note that the anticanonical line bundle of $S_{2} \times \mathbb{P}^{1}$ is not very ample.

\section{The Fano manifold $M_{10-1}$}

Mori-Mukai construction $S_{1} \times \mathbb{P}^{1}$.

Our construction $S_{1} \times \mathbb{P}^{1}$.

The quantum period Combining Corollary E.4 with Examples G.1 and G.11 yields

Regularizing gives

$$
G_{X}(t)=e^{-60 t} \sum_{l=0}^{\infty} \sum_{m=0}^{\infty} t^{l+2 m} \frac{(6 l) !}{(l !)^{2}(2 l) !(3 l) !(m !)^{2}} .
$$

$$
\begin{aligned}
\widehat{G}_{X}(t)=1+ & 10262 t^{2}+2021280 t^{3}+618997146 t^{4}+184490852160 t^{5} \\
+ & 57894898611620 t^{6}+18577980262739520 t^{7} \\
+ & 6078628630941923770 t^{8}+2017980469547810194560 t^{9}+\cdots .
\end{aligned}
$$

Minkowski period sequence None. Note that the anticanonical line bundle of $S_{1} \times \mathbb{P}^{1}$ is not very ample. 


\section{Conclusion}

This completes the calculation of the quantum periods for all 3-dimensional Fano manifolds, and the proof of Theorem A.1. It also completes the proof of our conjecture with Golyshev [11]: there is a one-to-one correspondence between deformation families of smooth 3-dimensional Fano manifolds $X$ with very ample anticanonical bundle and equivalence classes of Minkowski polynomials $f$ of manifold type such that the regularized quantum period $\widehat{G}_{X}$ of $X$ coincides with the period $\pi_{f}$ of $f$.

\section{A Fano manifold with non-unirational moduli space}

We conclude by giving an example of a Fano manifold $X$ such that the moduli space of $X$ is not unirational. The manifold $X$ has complex dimension 66 and, since unirationality of moduli spaces is a straightforward consequence of Theorem A.1, this example shows that the analogue of Theorem A.1 fails in dimension 66 . The same technique allows one to construct Fano manifolds $X_{3 k}$ of dimension $3 k$, for every $k \geq 22$, such that the moduli space of $X_{3 k}$ is not unirational. Let $C$ be a smooth curve of genus 23, let $L$ be a line bundle of degree 1 on $C$ and let $X$ be the moduli space of stable vector bundles over $C$ of rank 2 with fixed determinant $L$. It is known that $X$ is a non-singular projective variety [62] which is Fano [68]. The moduli space of $X$ is isomorphic to the moduli space of curves of genus 23 [71, Section 2], which has non-negative Kodaira dimension [32] and thus is not unirational.

\section{Appendix: Laurent polynomial mirrors for 3-dimensional Fano manifolds}

A table given in an online supplement [12] exhibits Laurent polynomial mirrors for each of the 105 deformation families of 3-dimensional Fano manifolds. The "Method" column summarizes the method by which we computed the quantum period in each case: "quantum Lefschetz" means "quantum Lefschetz with Fano ambient space and no mirror map"; "quantum Lefschetz with weak Fano ambient" means "quantum Lefschetz with non-Fano but weak Fano ambient space"; "quantum Lefschetz with mirror map" means "quantum Lefschetz with non-trivial mirror map"; the other entries should be self-explanatory. The "Minkowski ID" column records the ID in the Graded Ring Database [14] of the corresponding Minkowski period sequence of manifold type; there are only 98 non-trivial entries in this column as only the 98 deformation families of 3-dimensional Fano manifolds with very ample anticanonical bundle give rise to 
Minkowski polynomial mirrors. There are in general many Minkowski polynomials (and infinitely many other Laurent polynomials) mirror to a given 3-dimensional Fano manifold, but we have listed only one such Laurent polynomial in each case.

\section{References}

[1] M Akhtar, T Coates, S Galkin, A M Kasprzyk, Minkowski polynomials and mutations, SIGMA Symmetry Integrability Geom. Methods Appl. 8 (2012) MR3007265

[2] D Auroux, Mirror symmetry and T-duality in the complement of an anticanonical divisor, J. Gökova Geom. Topol. GGT 1 (2007) 51-91 MR2386535

[3] D Auroux, Special Lagrangian fibrations, wall-crossing, and mirror symmetry, from: "Geometry, analysis, and algebraic geometry: forty years of the Journal of Differential Geometry", (H-D Cao, S-T Yau, editors), Surv. Differ. Geom. 13, International Press, Somerville, MA (2009) 1-47 MR2537081

[4] V V Batyrev, Toric degenerations of Fano varieties and constructing mirror manifolds, from: "The Fano conference", (A Collino, A Conte, M Marchisio, editors), Univ. Torino, Turin (2004) 109-122 MR2112571

[5] A Beauville, Quantum cohomology of complete intersections, Mat. Fiz. Anal. Geom. 2 (1995) 384-398 MR1484335

[6] K Behrend, The product formula for Gromov-Witten invariants, J. Algebraic Geom. 8 (1999) 529-541 MR1689355

[7] K Behrend, B Fantechi, The intrinsic normal cone, Invent. Math. 128 (1997) 45-88 MR1437495

[8] A Bertram, I Ciocan-Fontanine, B Kim, Gromov-Witten invariants for abelian and nonabelian quotients, J. Algebraic Geom. 17 (2008) 275-294 MR2369087

[9] I Ciocan-Fontanine, B Kim, C Sabbah, The abelian/nonabelian correspondence and Frobenius manifolds, Invent. Math. 171 (2008) 301-343 MR2367022

[10] G Ciolli, Computing the quantum cohomology of some Fano threefolds and its semisimplicity, Boll. Unione Mat. Ital. Sez. B Artic. Ric. Mat. 7 (2004) 511-517 MR2072951

[11] T Coates, A Corti, S Galkin, V Golyshev, A Kasprzyk, Mirror symmetry and Fano manifolds, preprint (2012) arXiv:1212.1722 To appear in the Proceedings of the 6th European Congress of Mathematics

[12] T Coates, A Corti, S Galkin, A Kasprzyk, Table of Laurent polynomial mirrors of 3-dimensional Fano manifolds (2016) online supplement Available at http:// msp.org/gt/2016/20-1/gt-v20-n1-x03-laurent.pdf

[13] T Coates, A Corti, H Iritani, H-H Tseng, Computing genus-zero twisted GromovWitten invariants, Duke Math. J. 147 (2009) 377-438 MR2510741 
[14] T Coates, S Galkin, A Kasprzyk, 3d Minkowski period sequences, online resource Available at http://www.grdb.co.uk/forms/period3

[15] T Coates, A Gholampour, H Iritani, Y Jiang, P Johnson, C Manolache, The quantum Lefschetz hyperplane principle can fail for positive orbifold hypersurfaces, Math. Res. Lett. 19 (2012) 997-1005 MR3039825

[16] T Coates, A Givental, Quantum Riemann-Roch, Lefschetz and Serre, Ann. of Math. 165 (2007) 15-53 MR2276766

[17] T Coates, Y-P Lee, A Corti, H-H Tseng, The quantum orbifold cohomology of weighted projective spaces, Acta Math. 202 (2009) 139-193 MR2506749

[18] D A Cox, S Katz, Mirror symmetry and algebraic geometry, Mathematical Surveys and Monographs 68, Amer. Math. Soc. (1999) MR1677117

[19] B Fantechi, Stacks for everybody, from: "European Congress of Mathematics, I", (C Casacuberta, R M Miró-Roig, J Verdera, S Xambó-Descamps, editors), Progr. Math. 201, Birkhäuser, Basel (2001) 349-359 MR1905329

[20] S Galkin, Small toric degenerations of Fano threefolds, preprint (2007) Available at http://member.ipmu.jp/sergey.galkin/papers/std.pdf

[21] S Galkin, Toric degenerations of Fano manifolds, $\mathrm{PhD}$ thesis, Steklov Math. Institute (2008) In Russian Available at http:/www.mi.ras.ru/ galkin

[22] A Gathmann, Gromov-Witten invariants of blow-ups, J. Algebraic Geom. 10 (2001) 399-432 MR1832328

[23] A B Givental, Equivariant Gromov-Witten invariants, Internat. Math. Res. Notices (1996) 613-663 MR1408320

[24] A Givental, A mirror theorem for toric complete intersections, from: "Topological field theory, primitive forms and related topics", (M Kashiwara, A Matsuo, K Saito, I Satake, editors), Progr. Math. 160, Birkhäuser, Boston (1998) 141-175 MR1653024

[25] V V Golyshev, Classification problems and mirror duality, from: "Surveys in geometry and number theory: reports on contemporary Russian mathematics", (N Young, editor), London Math. Soc. Lecture Note Ser. 338, Cambridge Univ. Press (2007) 88-121 MR2306141

[26] M Gross, Toric degenerations and Batyrev-Borisov duality, Math. Ann. 333 (2005) 645-688 MR2198802

[27] M Gross, B Siebert, Affine manifolds, log structures, and mirror symmetry, Turkish J. Math. 27 (2003) 33-60 MR1975331

[28] M Gross, B Siebert, Mirror symmetry via logarithmic degeneration data, I, J. Differential Geom. 72 (2006) 169-338 MR2213573

[29] M Gross, B Siebert, Mirror symmetry via logarithmic degeneration data, II, J. Algebraic Geom. 19 (2010) 679-780 MR2669728 
[30] NP Gushel', Fano varieties of genus 8, Uspekhi Mat. Nauk 38 (1983) 163-164 MR693729 In Russian; translated in Russian Math. Surveys 38 (1983) 192-193

[31] NP Gushel', Fano 3-folds of genus 8, Algebra i Analiz 4 (1992) 120-134 MR1171957 In Russian; translated in St. Petersburg Math. J. 4 (1993) 115-129

[32] J Harris, D Mumford, On the Kodaira dimension of the moduli space of curves, Invent. Math. 67 (1982) 23-88 MR664324

[33] J Hu, Gromov-Witten invariants of blow-ups along points and curves, Math. Z. 233 (2000) 709-739 MR1759269

[34] J Hu, Gromov-Witten invariants of blow-ups along surfaces, Compositio Math. 125 (2001) 345-352 MR1818985

[35] H Iritani, An integral structure in quantum cohomology and mirror symmetry for toric orbifolds, Adv. Math. 222 (2009) 1016-1079 MR2553377

[36] V A Iskovskih, Fano threefolds, I, Izv. Akad. Nauk SSSR Ser. Mat. 41 (1977) 516-562 MR463151 In Russian; translated in Math. USSR-Izv. 11 (1977) 485-527

[37] V A Iskovskih, Fano threefolds, II, Izv. Akad. Nauk SSSR Ser. Mat. 42 (1978) 506-549 MR503430 In Russian; translated in Math. USSR-Izv. 12 (1978) 469-506

[38] V A Iskovskih, Anticanonical models of three-dimensional algebraic varieties, from: "Current problems in mathematics, XII", (R V Gamkrelidze, editor), VINITI, Moscow (1979) 59-157 MR537685 In Russian; translated in J. Soviet Math. 13 (1980) 745-814

[39] L Katzarkov, M Kontsevich, T Pantev, Hodge theoretic aspects of mirror symmetry, from: "From Hodge theory to integrability and TQFT tt*-geometry", (R Y Donagi, K Wendland, editors), Proc. Sympos. Pure Math. 78, Amer. Math. Soc. (2008) 87-174 MR2483750

[40] M Kontsevich, Enumeration of rational curves via torus actions, from: "The moduli space of curves", (R Dijkgraaf, C Faber, G van der Geer, editors), Progr. Math. 129, Birkhäuser, Boston (1995) 335-368 MR1363062

[41] M Kontsevich, Homological algebra of mirror symmetry, from: "Proceedings of the International Congress of Mathematicians, I”, (S D Chatterji, editor), Birkhäuser, Basel (1995) 120-139 MR1403918

[42] M Kontsevich, Y Manin, Gromov-Witten classes, quantum cohomology, and enumerative geometry, Comm. Math. Phys. 164 (1994) 525-562 MR1291244

[43] M Kontsevich, Y Soibelman, Homological mirror symmetry and torus fibrations, from: "Symplectic geometry and mirror symmetry", (K Fukaya, Y-G Oh, K Ono, G Tian, editors), World Sci. Publ., River Edge, NJ (2001) 203-263 MR1882331

[44] M Kontsevich, Y Soibelman, Affine structures and non-Archimedean analytic spaces, from: "The unity of mathematics", (P Etingof, V Retakh, I M Singer, editors), Progr. Math. 244, Birkhäuser, Boston (2006) 321-385 MR2181810 
[45] H-H Lai, Gromov-Witten invariants of blow-ups along submanifolds with convex normal bundles, Geom. Topol. 13 (2009) 1-48 MR2469512

[46] R Lazarsfeld, Positivity in algebraic geometry, II: Positivity for vector bundles, and multiplier ideals, Ergeb. Math. Grenzgeb. 49, Springer, Berlin (2004) MR2095472

[47] J Li, G Tian, Virtual moduli cycles and Gromov-Witten invariants of algebraic varieties, J. Amer. Math. Soc. 11 (1998) 119-174 MR1467172

[48] C Manolache, Virtual pull-backs, J. Algebraic Geom. 21 (2012) 201-245 MR2877433

[49] K Matsuki, Weyl groups and birational transformations among minimal models, Mem. Amer. Math. Soc. 557, Amer. Math. Soc. (1995) MR1259932

[50] S Mori, S Mukai, Classification of Fano 3-folds with $B_{2} \geq 2$, Manuscripta Math. 36 (1981/82) 147-162 MR641971 Correction in Manuscripta Math. 110 (2003) 407

[51] S Mori, S Mukai, On Fano 3-folds with $B_{2} \geq 2$, from: "Algebraic varieties and analytic varieties", (S Iitaka, editor), Adv. Stud. Pure Math. 1, North-Holland, Amsterdam (1983) 101-129 MR715648

[52] S Mori, S Mukai, Classification of Fano 3-folds with $B_{2} \geq 2$, I, from: "Algebraic and topological theories", (M Nagata, S Araki, A Hattori, editors), Kinokuniya, Tokyo (1986) 496-545 MR1102273

[53] S Mori, S Mukai, Extremal rays and Fano 3-folds, from: "The Fano Conference", (A Collino, A Conte, M Marchisio, editors), Univ. Torino, Turin (2004) 37-50 MR2112566

[54] S Mukai, Biregular classification of Fano 3-folds and Fano manifolds of coindex 3, Proc. Nat. Acad. Sci. USA 86 (1989) 3000-3002 MR995400

[55] S Mukai, Curves and symmetric spaces, Proc. Japan Acad. Ser. A Math. Sci. 68 (1992) 7-10 MR1158012

[56] S Mukai, Fano 3-folds, from: "Complex projective geometry", (G Ellingsrud, C Peskine, G Sacchiero, S A Strømme, editors), London Math. Soc. Lecture Note Ser. 179, Cambridge Univ. Press (1992) 255-263 MR1201387

[57] S Mukai, Curves and Grassmannians, from: "Algebraic geometry and related topics", (J-H Yang, Y Namikawa, editors), Conf. Proc. Lecture Notes Algebraic Geom. 1, Int. Press, Cambridge, MA (1993) 19-40 MR1285374

[58] S Mukai, Curves and symmetric spaces, I, Amer. J. Math. 117 (1995) 1627-1644 MR1363081

[59] S Mukai, New developments in Fano manifold theory related to the vector bundle method and moduli problems, Sūgaku 47 (1995) 125-144 MR1364825 In Japanese; translated in Sugaku Expositions 15 (1995) 125-150

[60] S Mukai, Curves and symmetric spaces, II, Ann. of Math. 172 (2010) 1539-1558 MR2726093 
[61] S Mukai, M Reid, H Takagi, Classification of indecomposable Gorenstein Fano 3folds, unpublished manuscript

[62] P E Newstead, Introduction to moduli problems and orbit spaces, Tata Institute of Fundamental Research Lectures on Mathematics and Physics 51, Narosa, New Delhi (1978) MR546290

[63] R Pandharipande, Rational curves on hypersurfaces (after A Givental), from: "Séminaire Bourbaki 1997/98 (Exposé 848)”, Astérisque 252, Soc. Math. France, Paris (1998) 307-340 MR1685628

[64] V V Przhiyalkovskiü, Gromov-Witten invariants of Fano threefolds of genera 6 and 8, Mat. Sb. 198 (2007) 145-158 MR2354283 In Russian; translated in Sb. Math. 198 (2007) 443-446

[65] V V Przhiyalkovskiur, Quantum cohomology of smooth complete intersections in weighted projective spaces and in singular toric varieties, Mat. Sb. 198 (2007) 107-122 MR2360793 In Russian; translated in Sb. Math. 198 (2007) 1325-1340

[66] V Przyjalkowski, On Landau-Ginzburg models for Fano varieties, Commun. Number Theory Phys. 1 (2007) 713-728 MR2412270

[67] M I Qureshi, Families of polarised varieties in weighted flag varieties, PhD thesis, University of Oxford (2011)

[68] S Ramanan, The moduli spaces of vector bundles over an algebraic curve, Math. Ann. 200 (1973) 69-84 MR0325615

[69] A Strangeway, A reconstruction theorem for quantum cohomology of Fano bundles on projective space, preprint (2013) arXiv:1302.5089v2

[70] A Strominger, S-T Yau, E Zaslow, Mirror symmetry is T-duality, Nuclear Phys. B 479 (1996) 243-259 MR1429831

[71] A N Tjurin, Geometry of moduli of vector bundles, Uspehi Mat. Nauk 29 (1974) 59-88 MR0404261 In Russian; translated in Russian Math. Surveys 29 (1974) 57-88

[72] A Vistoli, Intersection theory on algebraic stacks and on their moduli spaces, Invent. Math. 97 (1989) 613-670 MR1005008

TC, AC, AK: Department of Mathematics, Imperial College London 180 Queen's Gate, London SW72AZ, UK

SG: Faculty of Mathematics and Laboratory of Algebraic Geometry

National Research University Higher School of Economics

7 Vavilova str., Moscow, 117312, Russia

t.coates@imperial.ac.uk, a.corti@imperial.ac.uk,

Sergey.Galkin@phystech.edu, a.m.kasprzyk@imperial.ac.uk

Proposed: Jim Bryan

Seconded: Richard Thomas, Yasha Eliashberg
Received: 12 February 2014

Revised: 2 April 2015 